\title{
Thiopurines in Inflammatory Bowel Diseases : New perspectives to optimise safety and efficacy
}

Citation for published version (APA):

Wong, D. R. (2015). Thiopurines in Inflammatory Bowel Diseases : New perspectives to optimise safety and efficacy. [Doctoral Thesis, Maastricht University]. Datawyse / Universitaire Pers Maastricht. https://doi.org/10.26481/dis.20150109dw

Document status and date:

Published: 01/01/2015

DOI:

$10.26481 /$ dis.20150109dw

Document Version:

Publisher's PDF, also known as Version of record

\section{Please check the document version of this publication:}

- A submitted manuscript is the version of the article upon submission and before peer-review. There can be important differences between the submitted version and the official published version of record.

People interested in the research are advised to contact the author for the final version of the publication, or visit the DOI to the publisher's website.

- The final author version and the galley proof are versions of the publication after peer review.

- The final published version features the final layout of the paper including the volume, issue and page numbers.

Link to publication

\footnotetext{
General rights rights.

- You may freely distribute the URL identifying the publication in the public portal. please follow below link for the End User Agreement:

www.umlib.nl/taverne-license

Take down policy

If you believe that this document breaches copyright please contact us at:

repository@maastrichtuniversity.nl

providing details and we will investigate your claim.
}

Copyright and moral rights for the publications made accessible in the public portal are retained by the authors and/or other copyright owners and it is a condition of accessing publications that users recognise and abide by the legal requirements associated with these

- Users may download and print one copy of any publication from the public portal for the purpose of private study or research.

- You may not further distribute the material or use it for any profit-making activity or commercial gain

If the publication is distributed under the terms of Article $25 \mathrm{fa}$ of the Dutch Copyright Act, indicated by the "Taverne" license above, 


$$
\begin{aligned}
& \text { Thiopurines } \\
& \text { in Inflammatory } \\
& \text { Bowel Diseases }
\end{aligned}
$$

\section{NEW PERSPECTIVES \\ TO OPTIMISE SAFETY AND EFFICACY}

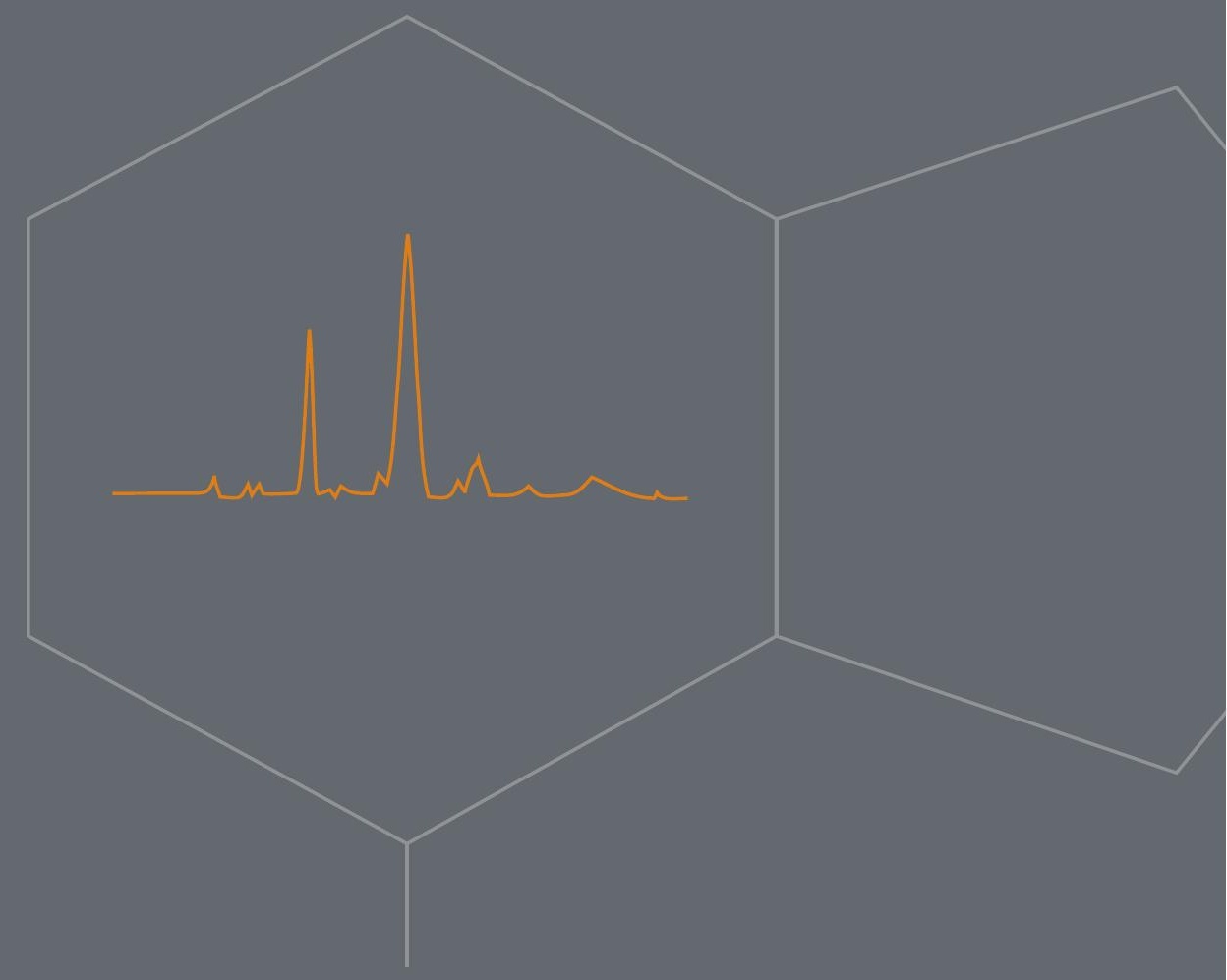

Dennis Roy Wong 
(C) Copyright Dennis Wong, Maastricht 2014

Cover: Molecular structure of 6-mercaptopurine; chromatogram of 6-TGN and 6MMPR Design: Marcel van Wintershoven

Layout: Tiny Wouters

Printing: Datawyse B.V.

ISBN 9789461593887 


\title{
Thiopurines in Inflammatory Bowel Diseases
}

\author{
New Perspectives to Optimise Safety and Efficacy
}

\author{
PROEFSCHRIFT \\ ter verkrijging van de graad van doctor aan de Universiteit Maastricht, \\ op gezag van de Rector Magnificus, Prof. dr. L.L.G. Soete, \\ volgens het besluit van het College van Decanen, \\ in het openbaar te verdedigen op \\ vrijdag 9 januari 2015 om 14:00 uur \\ door
}

Dennis Roy Wong

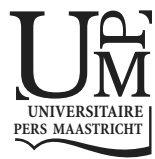




\section{Promotores}

Prof. dr. A.A.M. Masclee

Prof. dr. C. Neef

\section{Copromotores}

Dr. P.M. Hooymans

Dr. L.G.J.B. Engels

\section{Beoordelingscommissie}

Prof. dr. A. Bast, voorzitter

Prof. dr. J.R.B.J. Brouwers, Rijksuniversiteit Groningen

Prof. dr. G. Dijkstra, UMC Groningen

Dr. M. Pierik

Prof. dr. F.J. van Schooten 
Using no way as way, having no limitation as limitation.

Bruce Lee (1940 - 1973)

voor mijn ouders voor Emmely 


\section{Contents}

Chapter 1 General introduction and Outline of the thesis 9

PART I Pharmacogenetics of thiopurines in inflammatory bowel diseases

Chapter 2 Pharmacogenetics of thiopurines in inflammatory bowel disease

Curr Pharm Des. 2010;16:145-154

Chapter 3 Screening for TPMT genotype followed by personalised dosing of azathioprine and mercaptopurine in inflammatory bowel disease patients: a randomised trial Submitted

Chapter 4 The role of xanthine oxidase in thiopurine metabolism: a case report 69 Ther Drug Monit. 2007;29:845-848

PART II Therapeutic drug monitoring of thiopurine metabolites to optimise 77 efficacy and safety in inflammatory bowel diseases

Chapter 5 Therapeutic drug monitoring of thiopurine metabolites in adult thiopurine tolerant IBD patients on maintenance therapy J Crohns Colitis 2012;6:698-707

Chapter 6 Early assessment of thiopurine metabolites identifies patients at risk of leukopenia in inflammatory bowel disease submitted

Chapter 7 Early elevated 6-methylmercaptopurine levels predict thiopurineinduced hepatotoxicity in inflammatory bowel disease Submitted

Chapter 8 Safe 6-thioguanine therapy of a TPMT deficient Crohn's disease patient by using therapeutic drug monitoring J Crohns Colitis 2009;3:128-130 
Chapter 9 Dose-dependent influence of 5-aminosalicylates on thiopurine metabolism

Am J Gastroenterol 2007;102:2747-2753

Chapter 10 Influence of 5-aminosalicylic acid on 6-thioguanosine phosphate 157 metabolite levels: a prospective study in patients under steady thiopurine therapy Br J Pharmacol. 2010;160:1083-1091

Chapter 11 The pharmacokinetic effect of adalimumab on thiopurine metabolism 173 in Crohn's disease patients J Crohns Colitis 2014;8:120-128

Chapter 12 General discussion and future perspectives 189

Chapter 13 Conclusions and clinical recommendations

Summary

Samenvatting 235

Valorisation 245

Dankwoord 253

List of publications 263

Curriculum vitae 



\section{Chapter 1}

Introduction and outline of the thesis 
10 Chapter 1 


\section{Introduction and outline of the thesis}

The inflammatory bowel diseases (IBD) Crohn's disease (CD), ulcerative colitis (UC), and indeterminate colitis are chronic, relapsing inflammatory disorders, predominantly affecting the gastrointestinal tract. UC is characterised by diffuse mucosal inflammation limited to the colon, whereas CD is characterised by patchy, transmural inflammation, which may affect any part of the gastrointestinal tract, although extraintestinal manifestations (e.g. mouth, skin or joints) are not uncommon. ${ }^{1-3}$

In recent decades, both the incidence and prevalence of IBD are gradually increasing, particularly in developed countries worldwide. The highest incidence rates and prevalence are seen in North America and Europe, arising from interactions between genetic and environmental factors, such as microbial exposures, sanitation, occupations, diet, lifestyle behaviours, medication, and pollution exposures. ${ }^{1-3}$

The main initial goal of treatment in IBD is to induce and maintain remission of disease, and eventually, to achieve mucosal healing and a reduction in the need for hospital admission and surgery. Causal medical therapy to cure these diseases is not yet available, since the exact aetiology of these diseases is currently unknown. ${ }^{1,2}$

Mesalazine (aminosalicylates, 5-ASA) is a safe, well-tolerated and effective drug in patients with mild-to-moderate UC, but the benefit in $C D$ is limited. ${ }^{4-6}$ The immunosuppressive corticosteroids are highly effective for the induction of remission, but chronic use for maintenance of remission is limited with respect to efficacy and due to occurrence of significant side-effects. In order to maintain clinical remission, patients with moderate-severe IBD who initially respond well to corticosteroid therapy are often treated with steroid-sparing immunomodulators, such as the thiopurine drugs, azathioprine (AZA) or mercaptopurine (6MP), or methotrexate (MTX), which is mainly effective in CD. The pro-inflammatory cytokine tumor necrosis factor- $\alpha$ (TNF- $\alpha$ ), produced by macrophages and activated T-cells, plays a key role in the pathophysiology of inflammatory bowel diseases. Monoclonal antibodies directed against TNF- $\alpha$ are used to treat patients with moderate-to-severe IBD and/or patients who are refractory to conventional therapy. ${ }^{5,6}$ The anti-TNF monoclonal antibodies infliximab (IFX) and adalimumab (ADA) have proven to be effective, and have been approved in the Netherlands for the use of induction of remission and maintenance of remission of CD. ${ }^{7-10}$ IFX, ADA, and golimumab have been approved for the induction and maintenance of remission of UC. ${ }^{11-14}$

The steroid-sparing thiopurine agents have already been used for over 50 years in the treatment of IBD. In Europe, azathioprine is the only registered thiopurine for these indications. Mercaptopurine has officially been approved for IBD in the US, but is frequently prescribed off-label in the Netherlands.

Nowadays, the purine analogues are the most frequently used immunosuppressive drugs in IBD and have proven to be effective in active disease to induce remission, but also for the maintenance of clinical remission of both $C D$ and UC. Therefore thiopurine 
agents are considered to be the mainstay of IBD medical therapy, especially in steroiddependent and steroid-refractory patients. ${ }^{15-17}$

The onset of action of thiopurines is usually slow (8-16 weeks), which makes these drugs not suitable for fast induction treatment in the setting of patients with an acute flare of their IBD. ${ }^{15,18}$

The most frequently reported thiopurine-associated adverse events are nausea, hepatotoxicity, myelotoxicity, and pancreatitis. ${ }^{19,20}$ Thiopurine side effects are classified into dose-independent (i.e. 'allergic/idiosyncratic' reactions) and pharmacologically explainable 'dose-dependent' side effects. ${ }^{21}$ The most commonly reported doseindependent adverse drug events are skin rash, pancreatitis and flu-like symptoms (e.g. fever and arthralgia) and hepatitis. The most important dose-dependent adverse events are nausea, malaise and myelosuppression, but hepatotoxicity may also be dosedependent. $^{21,22}$ In clinical practice, in up to two-thirds of IBD-patients the initial therapeutic goal (e.g. mucosal healing, tapering or elimination of corticosteroids, healing of fistulas or abscesses, pain relief, etc.) is achieved. ${ }^{23}$ After an initial response the efficacy is reasonably well sustained with remission rates of $95 \%, 69 \%$ and $55 \%$ after 1,3 and 5 years, respectively. $^{24}$

However, side effects occur in up to $20-30 \%$ of patients using thiopurines. ${ }^{20}$ Subsequently, up to a quarter of the patients discontinue thiopurine treatment due to intolerable adverse events within the first 3 months of thiopurine therapy. ${ }^{19,21}$

The alternative thiopurine drug 6-thioguanine (6TG) may be considered in stringently defined indications in IBD as an off-label rescue drug in patients who fail due to intolerance or resistance to 5-ASA, the conventional thiopurines AZA or 6MP, methotrexate and anti-TNF therapy. ${ }^{25,26}$

Neither AZA nor 6MP have intrinsic pharmacological activity. AZA is a pro-drug that is converted to $6 \mathrm{MP}$ in the liver. $6 \mathrm{MP}$ subsequently undergoes extensive metabolic transformations, yielding to a variety of pharmacological active metabolites. ${ }^{27}$

The observed interindividual differences in therapeutic response and thiopurine-related adverse reactions or toxicity are attributed to a large extent to a variation of the formation of these active metabolites, which is partly related to genetic variants in the genes encoding for the various enzymes involving the thiopurine metabolism. The most important thiopurine metabolites are considered to be the 6-thioguanine nucleotides (6-TGN) and the methylated 6-methylmercaptopurine ribonucleotides (6-MMPR).

One of the key enzymes in the delicate balance between 6-MMPR and 6-TGN metabolite formation is thiopurine S-methyltransferase (TPMT). TPMT enzyme activity is related to genetic variants of the TPMT gene. Individuals carrying variant alleles show reduced TPMT enzyme activity, and once treated with AZA or 6MP high 6-TGN concentrations will be formed resulting in an increased risk of leukopenia. ${ }^{28}$ The metabolism of 6TG is much less complex yielding to formation of the active metabolite 6-TGN without generation of 6-MMPR. ${ }^{26}$ 
The proposed thiopurine metabolism of the thiopurines is presented in Figure $1.1 .^{29}$ Concentrations of 6-TGN and 6-MMPR can be measured in erythrocytes, as a surrogate marker of the actual target cells, the leukocytes. ${ }^{27}$

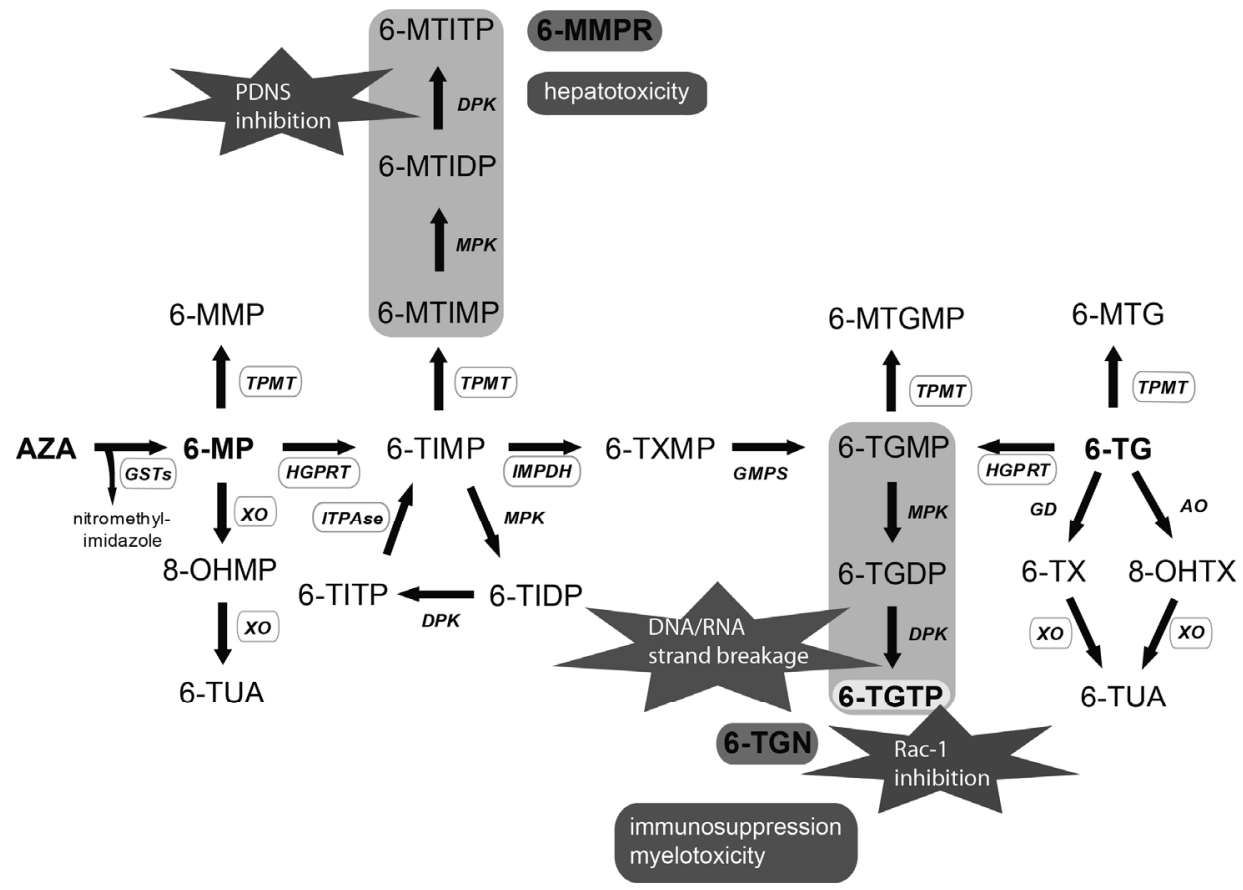

Figure 1.1 AZA, azathioprine; 6-MP, mercaptopurine; 6-MMP, 6-methylmercaptopurine; 8-OHMP, 8-hydroxy-6-mercaptopurine; 6-TUA, 6-thiouric acid; 6-MTIMP, 6-methylthioinosine monophosphate; 6-MTIDP, 6-methylthioinosine diphosphate; 6-MTITP, 6-methylthioinosine triphosphate; 6-TIMP, 6-thioinosine monophosphate; 6-TIDP, 6-thioinosine diphosphate; 6-TITP, 6-thioinosine triphosphate; 6-TXMP, 6-thioxanthosine monophosphate; 6-TGMP, 6-thioguanine monophosphate; 6-TGDP, 6-thioguanine diphosphate; 6-TGTP, 6-thioguanine triphosphate; 6-MTGMP, 6-methylthioguanine monophosphate; 6-TG, 6-thioguanine; 6-MTG, 6-methyl-thioguanine; $X O$, xanthine oxidase; TPMT, thiopurine S-methyl transferase; HGPRT, hypoxanthine-guanine phosphoribosyl transferase; IMPDH, inosine monophosphate dehydrogenase; GMPS, guanosine monophosphate synthetase; MPK, monophosphate kinase; DPK, diphosphate kinase; ITPase, inosine triphosphate pyrophosphatase; 6-TX, 6-thioxanthine; 8-OHTX, 8-hydroxy-6-thioxanthine; GD, guanine deaminase; AO, aldehyde oxidase. PDNS, purine de novo synthesis; DNA, deoxyribonucleic acid; RNA, ribonucleic acid. 6-MTIMP, 6-MTIDP and 6-MTITP together form the 6-methyl- mercaptopurine ribonucleotides (6-MMPR). 6-TGMP, 6-TGDP and 6-TGTP form together the 6-thioguanine nucleotides (6-TGN). 
The 6-TGN metabolites are responsible for the immunosuppressive effects, but highly elevated concentrations have been associated with the development of myelotoxicity. The therapeutic range in steady-state has been defined as a 6-TGN concentration between 235 and $490 \mathrm{pmol} / 8 \times 10^{8}$ red blood cells (RBC). ${ }^{30-32}$ 6-MMPR concentrations higher than $5,700 \mathrm{pmol} / 8 \times 10^{8} \mathrm{RBC}$ are associated with hepatotoxicity and treatment failure. $^{30,32,33}$ On the other hand, several studies have not been able to demonstrate a relationship between thiopurine metabolite concentrations and therapeutic response or toxicity. ${ }^{34-38}$

Measurement of the active thiopurine metabolite concentrations, or so-called therapeutic drug monitoring (TDM), can be used to explain toxicity or in case of insufficient therapeutic response to evaluate suboptimal dosing or non-compliance in order to optimise thiopurine therapy.

In daily practice, thiopurines are dosed based on body weight (AZA $(2.0-2.5 \mathrm{mg} / \mathrm{kg} / \mathrm{day}$ ) and $6 \mathrm{MP}(1.0-1.5 \mathrm{mg} / \mathrm{kg} / \mathrm{day}), 6 \mathrm{TG}(0.3 \mathrm{mg} / \mathrm{kg} /$ day, max. $25 \mathrm{mg} / \mathrm{day}))$, although there is no clear relation between the thiopurine dose and active metabolite concentration due to a large inter-patient variability. ${ }^{5,6,26,33}$

Unfortunately, up to one-fourth of the patients discontinue therapy due to intolerable adverse reactions already during the first months of treatment. In addition, thiopurines are not effective in one-third of the patients. Since the introduction of the fast acting anti-TNF- $\alpha$ agents in the treatment of $C D$ and, to a lesser extent, UC, the role of thiopurines in IBD is evolving.

Considering the loss of response of anti-TNF therapy the limited number of available other effective immunosuppressive drugs in IBD and taking into account the high costs of the novel immunomodulating drugs, it is important to optimise conventional IBD therapy, especially thiopurine therapy. ${ }^{39}$

In the last decades, new insights obtained by pharmacogenetics in thiopurine metabolism and assessment of 6-TGN and 6-MMPR metabolites in patients on thiopurine therapy have led to strategies to reduce toxicity and optimise efficacy and safety in IBD.

\section{Outline of the thesis}

The objectives of the clinical studies performed within the scope of this thesis were to find new ways to improve efficacy and safety of thiopurine therapy by assessment of thiopurine metabolite concentrations in patients with inflammatory bowel diseases.

First, in part $I$, the clinical relevance of pharmacogenetics in thiopurine metabolism is studied. In Chapter 2, several polymorphisms of genes encoding for important enzymes involving thiopurine metabolism are reviewed and the clinical implications in IBD are discussed in detail. Chapter 3 describes the results of a large nationwide prospective 
randomised-controlled trial evaluating whether TPMT genotype-based thiopurine dosing prior to treatment reduces the risk of leukopenia in IBD patients starting thiopurine therapy. Chapter 4 describes the case of a female patient with chronic autoimmune pancreatitis treated with high-dose of azathioprine. This case report demonstrates the relevance of the enzyme xanthine oxidase in the biotransformation of thiopurines.

In part II, the usefulness of thiopurine metabolite measurement to optimise efficacy and safety is evaluated. Chapter 5 describes a prospective cross-sectional study in adult IBD patients on thiopurine maintenance therapy comparing the thiopurine metabolite concentrations in patients with a flare of disease and patients in remission. The therapeutic threshold of 6-TGN was determined and compared with previously reported metabolite threshold concentrations.

Thiopurine-induced leukopenia has been attributed to highly elevated 6-TGN steadystate concentrations. Chapter 6 describes a prospective study on the predictive value of 6-TGN and 6-MMPR metabolites, assessed one week after initiation of therapy, for the development of leukopenia.

Up to one-fourth of patients discontinue thiopurine therapy during the first months due to intolerable adverse events. Chapter 7 describes a prospective study on the predictive value of the 6-MMPR metabolite concentration at week one for the development of hepatotoxicity, gastrointestinal complaints and general malaise. Chapter 8 reports on the case of a TPMT deficient patient with Crohn's disease and demonstrates how the alternative thiopurine 6-thioguanine can successfully be used, guided and optimised by frequent thiopurine metabolite assessment.

In part III, two thiopurine pharmacokinetic interactions are evaluated. Previous studies indicated that 5-aminosalicylates (5-ASA) and the anti-TNF agent infliximab may influence thiopurine metabolism. Chapter 9 describes a prospective study on the influence of two different 5-ASA dosages on thiopurine metabolism. Chapter 10 describes the influence of the two 5-ASA dosages on the individual 6-thioguanine phosphate metabolite concentrations. Chapter 11 describes a prospective study to determine the effect of the anti-TNF agent adalimumab on thiopurine metabolism in Crohn's disease patients.

In Chapter 12, the relevant findings of the studies of this thesis are reviewed, and in Chapter 13 implications for clinical practice and future research are discussed. Finally, the relevant findings of the studies are summarised both in the English and Dutch language, successively. 


\section{References}

1. Van Assche G, Dignass A, Panes J, Beaugerie L, Karagiannis J, Allez M, Ochsenkühn T, Orchard T, Rogler G, Louis E, Kupcinskas L, Mantzaris G, Travis S, Stange E; European Crohn's and Colitis Organisation (ECCO). The second European evidence-based Consensus on the diagnosis and management of Crohn's disease: Definitions and diagnosis. J Crohns Colitis 2010;4:7-27.

2. Dignass A, Eliakim R, Magro F, Maaser C, Chowers Y, Geboes K, Mantzaris G, Reinisch W, Colombel JF, Vermeire S, Travis S, Lindsay JO, Van Assche G. Second European evidence-based consensus on the diagnosis and management of ulcerative colitis part 1: definitions and diagnosis. J Crohns Colitis 2012;6:965-90.

3. Molodecky NA, Soon IS, Rabi DM, Ghali WA, Ferris M, Chernoff G, Benchimol El, Panaccione R, Ghosh S, Barkema HW, Kaplan GG. Increasing incidence and prevalence of the inflammatory bowel diseases with time, based on systematic review. Gastroenterology 2012;142:46-54 e42; quiz e30.

4. Williams C, Panaccione R, Ghosh S, Rioux K. Optimizing clinical use of mesalazine (5-aminosalicylic acid) in inflammatory bowel disease. Therap Adv Gastroenterol 2011;4:237-48.

5. Dignass A, Van Assche G, Lindsay JO, Lémann M, Söderholm J, Colombel JF, Danese S, D'Hoore A, Gassull M, Gomollón F, Hommes DW, Michetti P, O'Morain C, Oresland T, Windsor A, Stange EF, Travis SP; European Crohn's and Colitis Organisation (ECCO). The second European evidence-based Consensus on the diagnosis and management of Crohn's disease: Current management. J Crohns Colitis 2010;4: 28-62.

6. Dignass A, Lindsay JO, Sturm A, Windsor A, Colombel JF, Allez M, D'Haens G, D'Hoore A, Mantzaris G, Novacek G, Oresland T, Reinisch W, Sans M, Stange E, Vermeire S, Travis S, Van Assche G. Second European evidence-based consensus on the diagnosis and management of ulcerative colitis part 2: current management. J Crohns Colitis 2012;6:991-1030.

7. Colombel JF, Sandborn WJ, Rutgeerts P, Enns R, Hanauer SB, Panaccione R, Schreiber S, Byczkowski D, Li J, Kent JD, Pollack PF. Adalimumab for maintenance of clinical response and remission in patients with Crohn's disease: the CHARM trial. Gastroenterology 2007;132:52-65.

8. Colombel JF, Sandborn WJ, Rutgeerts P, Kamm MA, Yu AP, Wu EQ, Pollack PF, Lomax KG, Chao J, Mulani PM. Comparison of two adalimumab treatment schedule strategies for moderate-to-severe Crohn's disease: results from the CHARM trial. Am J Gastroenterol 2009;104:1170-9.

9. Hanauer SB, Feagan BG, Lichtenstein GR, Mayer LF, Schreiber S, Colombel JF, Rachmilewitz D, Wolf DC, Olson A, Bao W, Rutgeerts P; ACCENT I Study Group. Maintenance infliximab for Crohn's disease: the ACCENT I randomised trial. Lancet 2002;359:1541-9.

10. Targan SR, Hanauer SB, van Deventer SJ, Mayer L, Present DH, Braakman T, DeWoody KL, Schaible TF, Rutgeerts PJ. A short-term study of chimeric monoclonal antibody CA2 to tumor necrosis factor alpha for Crohn's disease. Crohn's Disease cA2 Study Group. N Engl J Med 1997;337:1029-35.

11. Rutgeerts P, Sandborn WJ, Feagan BG, Reinisch W, Olson A, Johanns J, Travers S, Rachmilewitz D, Hanauer SB, Lichtenstein GR, de Villiers WJ, Present D, Sands BE, Colombel JF. Infliximab for induction and maintenance therapy for ulcerative colitis. N Engl J Med 2005;353:2462-76.

12. Sandborn WJ, van Assche G, Reinisch W, Colombel JF, D'Haens G, Wolf DC, Kron M, Tighe MB, Lazar A, Thakkar RB. Adalimumab induces and maintains clinical remission in patients with moderate-to-severe ulcerative colitis. Gastroenterology 2012;142:257-65 e1-3.

13. Sandborn WJ, Feagan BG, Marano C, Zhang H, Strauss R, Johanns J, Adedokun OJ, Guzzo C, Colombel JF, Reinisch W, Gibson PR, Collins J, Järnerot G, Rutgeerts P, PURSUIT-Maintenance Study Group.. Subcutaneous golimumab maintains clinical response in patients with moderate-to-severe ulcerative colitis. Gastroenterology 2014;146:96-109 e1.

14. Sandborn WJ, Feagan BG, Marano C, Zhang H, Strauss R, Johanns J, Adedokun OJ, Guzzo C, Colombel JF, Reinisch W, Gibson PR, Collins J, Järnerot G, Hibi T, Rutgeerts P, PURSUIT-SC Study Group. Subcutaneous golimumab induces clinical response and remission in patients with moderate-to-severe ulcerative colitis. Gastroenterology 2014;146:85-95; quiz e14-5.

15. Prefontaine E, Macdonald JK, Sutherland LR. Azathioprine or 6-mercaptopurine for induction of remission in Crohn's disease. Cochrane Database Syst Rev 2009(4):CD000545.

16. Prefontaine E, Sutherland LR, Macdonald JK, Cepoiu M. Azathioprine or 6-mercaptopurine for maintenance of remission in Crohn's disease. Cochrane Database Syst Rev 2009(1):CD000067. 
17. Timmer A, McDonald JW, Macdonald JK. Azathioprine and 6-mercaptopurine for maintenance of remission in ulcerative colitis. Cochrane Database Syst Rev 2007(1):CD000478.

18. Pearson DC, May GR, Fick GH, Sutherland LR. Azathioprine and 6-mercaptopurine in Crohn disease. A meta-analysis. Ann Intern Med 1995;123:132-42.

19. Jharap B, Seinen ML, de Boer NK, van Ginkel JR, Linskens RK, Kneppelhout JC, Mulder CJ, van Bodegraven AA. Thiopurine therapy in inflammatory bowel disease patients: analyses of two 8-year intercept cohorts. Inflamm Bowel Dis 2010;16:1541-9.

20. Chaparro M, Ordás I, Cabré E, Garcia-Sanchez V, Bastida G, Peñalva M, Gomollón F, García-Planella E, Merino O, Gutiérrez A, Esteve M, Márquez L, Garcia-Sepulcre M, Hinojosa J, Vera I, Muñoz F, Mendoza JL, Cabriada JL, Montoro MA, Barreiro-de Acosta M, Ceña G, Saro C, Aldeguer X, Barrio J, Maté J, Gisbert JP. Safety of thiopurine therapy in inflammatory bowel disease: long-term follow-up study of 3931 patients. Inflamm Bowel Dis 2013;19:1404-10.

21. de Jong DJ, Derijks LJ, Naber AH, Hooymans PM, Mulder CJ. Safety of thiopurines in the treatment of inflammatory bowel disease. Scand J Gastroenterol Suppl 2003(239):69-72.

22. Gisbert JP, Gonzalez-Lama Y, Mate J. Thiopurine-induced liver injury in patients with inflammatory bowel disease: a systematic review. Am J Gastroenterol 2007;102:1518-27.

23. Korelitz BI, Adler DJ, Mendelsohn RA, Sacknoff AL. Long-term experience with 6-mercaptopurine in the treatment of Crohn's disease. Am J Gastroenterol 1993;88:1198-205.

24. Fraser AG, Orchard TR, Jewell DP. The efficacy of azathioprine for the treatment of inflammatory bowel disease: a 30 year review. Gut 2002;50:485-9.

25. de Boer NK, van Asseldonk DP, van Bodegraven A. ECCO consensus: evidence-based use of 6thioguanine therapy in Crohn's disease? J Crohns Colitis 2010;4:484-5.

26. de Boer NK1, Reinisch W, Teml A, van Bodegraven AA, Schwab M, Lukas M, Ochsenkühn T, Petritsch W, Knoflach P, Almer S, van der Merwe SW, Herrlinger KR, Seiderer J, Vogelsang H, Mulder CJ; Dutch 6-TG working group. 6-Thioguanine treatment in inflammatory bowel disease: a critical appraisal by a European 6-TG working party. Digestion 2006;73:25-31.

27. Lennard L. The clinical pharmacology of 6-mercaptopurine. Eur J Clin Pharmacol 1992;43:329-39.

28. Colombel JF, Ferrari N, Debuysere H, Marteau P, Gendre JP, Bonaz B, Soulé JC, Modigliani R, Touze Y, Catala P, Libersa C, Broly F. Genotypic analysis of thiopurine S-methyltransferase in patients with Crohn's disease and severe myelosuppression during azathioprine therapy. Gastroenterology 2000;118:1025-30.

29. Derijks LJ, Wong DR. Pharmacogenetics of thiopurines in inflammatory bowel disease. Curr Pharm Des 2010;16:145-54.

30. Cuffari C, Seidman EG, Latour S, Theoret Y. Quantitation of 6-thioguanine in peripheral blood leukocyte DNA in Crohn's disease patients on maintenance 6-mercaptopurine therapy. Can J Physiol Pharmacol 1996;74:580-5.

31. Osterman MT, Kundu R, Lichtenstein GR, Lewis JD. Association of 6-thioguanine nucleotide levels and inflammatory bowel disease activity: a meta-analysis. Gastroenterology 2006;130:1047-53.

32. Dubinsky MC, Lamothe S, Yang HY, Targan SR, Sinnett D, Théorêt Y, Seidman EG. Pharmacogenomics and metabolite measurement for 6-mercaptopurine therapy in inflammatory bowel disease. Gastroenterology 2000;118:705-13.

33. Derijks LJ, Gilissen LP, Engels LG, Bos LP, Bus PJ, Lohman JJ, Curvers WL, Van Deventer SJ, Hommes DW, Hooymans PM. Pharmacokinetics of 6-mercaptopurine in patients with inflammatory bowel disease: implications for therapy. Ther Drug Monit 2004;26:311-8.

34. Lowry PW, Franklin CL, Weaver AL, Szumlanski CL, Mays DC, Loftus EV, Tremaine WJ, Lipsky JJ, Weinshilboum RM, Sandborn WJ. Leucopenia resulting from a drug interaction between azathioprine or 6-mercaptopurine and mesalamine, sulphasalazine, or balsalazide. Gut 2001;49:656-64.

35. Belaiche J, Desager JP, Horsmans Y, Louis E. Therapeutic drug monitoring of azathioprine and 6mercaptopurine metabolites in Crohn disease. Scand J Gastroenterol 2001;36:71-6.

36. Dubinsky MC, Yang H, Hassard PV, Seidman EG, Kam LY, Abreu MT, Targan SR, Vasiliauskas EA. 6-MP metabolite profiles provide a biochemical explanation for 6-MP resistance in patients with inflammatory bowel disease. Gastroenterology 2002;122:904-15.

37. Wright S, Sanders DS, Lobo AJ, Lennard L. Clinical significance of azathioprine active metabolite concentrations in inflammatory bowel disease. Gut 2004;53:1123-8. 
38. Goldenberg BA, Rawsthorne P, Bernstein CN. The utility of 6-thioguanine metabolite levels in managing patients with inflammatory bowel disease. Am J Gastroenterol 2004;99:1744-8.

39. Taylor KM, Irving PM. Optimization of conventional therapy in patients with IBD. Nat Rev Gastroenterol Hepatol 2011;8:646-56. 


\section{Part}

Pharmacogenetics of thiopurines in inflammatory bowel diseases 



\section{Chapter 2}

Pharmacogenetics of thiopurines in inflammatory bowel disease

LJ Derijks, DR Wong

Curr Pharm Des. 2010;16:145-154 


\section{Abstract}

Thiopurines are widely used in the treatment of inflammatory bowel disease (IBD). However, in clinical practice azathioprine (AZA) or 6-mercaptopurine (6-MP) are not effective in one-third of patients and up to one-fifth of patients discontinues thiopurine therapy due to adverse reactions. The observed interindividual differences in therapeutic response and toxicity to thiopurines are to a large extent explained by the variable formation of active metabolites, which is at least partly caused by genetic polymorphisms of the genes encoding crucial enzymes in thiopurine metabolism. In this in depth review, we will discuss the genetic polymorphisms of genes encoding for glutathione S-tranferases, xanthine oxidase, thiopurine S-methyltransferase, inosine triphosphate pyrophosphatase, hypoxanthine phosphoribosyltransferase, inosine monophosphate dehydrogenase and multidrug resistance proteins. Pharmacogenetic knowledge in this field has increased dramatically and is still rapidly increasing, but the translation into practical guidelines with tailored advices will take much effort in the near future. 


\section{Introduction}

As early as the 1950s, Gertrude B. Elion, a female biochemical scientist from New York, developed 6-mercaptopurine (6-MP), 6-thioguanine (6-TG) and azathioprine (AZA), as chemotherapeutic agents for acute or chronic lymphoblastic leukaemia and as immunosuppressants for prevention of rejection after solid organ transplantation. In 1988, Elion, amongst others, was awarded the Nobel Prize in Physiology or Medicine for her discoveries. Nowadays, thiopurines are most frequently used in inflammatory bowel diseases (IBD) and have proven to be effective for both inducing and maintaining long-term remission in Crohn's disease (CD) and ulcerative colitis (UC). ${ }^{1-3}$ In clinical practice however, AZA and 6-MP, which both have a relatively narrow therapeutic index, are not effective in one-third of patients and up to one-fifth of patients discontinues thiopurine therapy due to adverse reactions. Interindividual differences in both efficacy and toxicity are to a large extent attributed to interindividual differences in thiopurine metabolism, which after almost 50 years we start understanding.

Neither AZA nor 6-MP has intrinsic activity, hence both drugs must undergo extensive metabolic transformations (Figure 2.1). AZA is a pro-drug, that is rapidly and almost completely (88\%) converted to 6-MP and methylnitroimidazole through a partly nonenzymatic reaction in liver and gut. The latter may have immunosuppressive properties of its own, but also causes intolerance in a considerable group of patients, $48-75 \%$ of which successfully switches to 6-MP. ${ }^{4,5}$ The remaining $12 \%$ of AZA yields hypoxanthine and methyl nitrothioimidazole. ${ }^{6}$ Following intracellular uptake, 6-MP is further metabolised by three competitive enzymes (Figure 2.1); two of those, xanthine oxidase (XO) and thiopurine S-methyltransferase (TPMT) are catabolic, and one, hypoxanthineguanine phosphoribosyltransferase (HGPRT), is anabolic. ${ }^{6}$ XO oxidises 6-MP to 8-hydroxy-6-mercaptopurine (8-OHMP) and 6-thiouric acid (6-TUA) subsequently, whereas TPMT methylates 6-MP to 6-methylmercaptopurine (6-MMP). HGPRT carries out the first anabolic step to produce 6-thioinosine 5'-monophosphate (6-TIMP), which is further transformed in a rate-limiting step by inosine monophosphate dehydrogenase (IMPDH) into 6-thioxanthosine monophosphate (6-TXMP). 6-TXMP, in turn, is metabolised by guanosine monophosphate synthetase (GMPS) to 6-thioguanine monophosphate (6-TGMP), -diphosphate (6-TGDP) and -triphosphate (6-TGTP), together the so-called 6-thioguanine nucleotides (6-TGN). 6-TIMP can alternatively be methylated by TPMT, yielding 6-methyl thioinosine monophosphate (6-MTIMP), -diphosphate (6-MTIDP) and -triphosphate (6-MTITP), the so-called 6-methylmercaptopurine ribonucleotides (6-MMPR). Finally, it is hypothesised that 6-TIMP is converted successively into 6-thioinosine diphosphate (6-TIDP) and -triphosphate (6-TITP) to form 6-TIMP again by the enzyme inosine triphosphate pyrophosphatase (ITPase). ${ }^{7}$. In contrast to AZA and 6-MP, the metabolism of 6-TG is much less complicated: HGPRT directly converts 6-TG to 6-TGMP and ultimately the 6-TGN. The competing enzymes TPMT, guanine deaminase (GD) and aldehyde oxidase (AO) yield 6-methylthioguanine (6-MTG), 6-thioxanthine (6-TX) and 8-hydroxy-6- 
thioguanine (8-OHTG) respectively. Finally, XO metabolises 6-TX and 8-OHTG to form 6-TUA. ${ }^{8,9}$

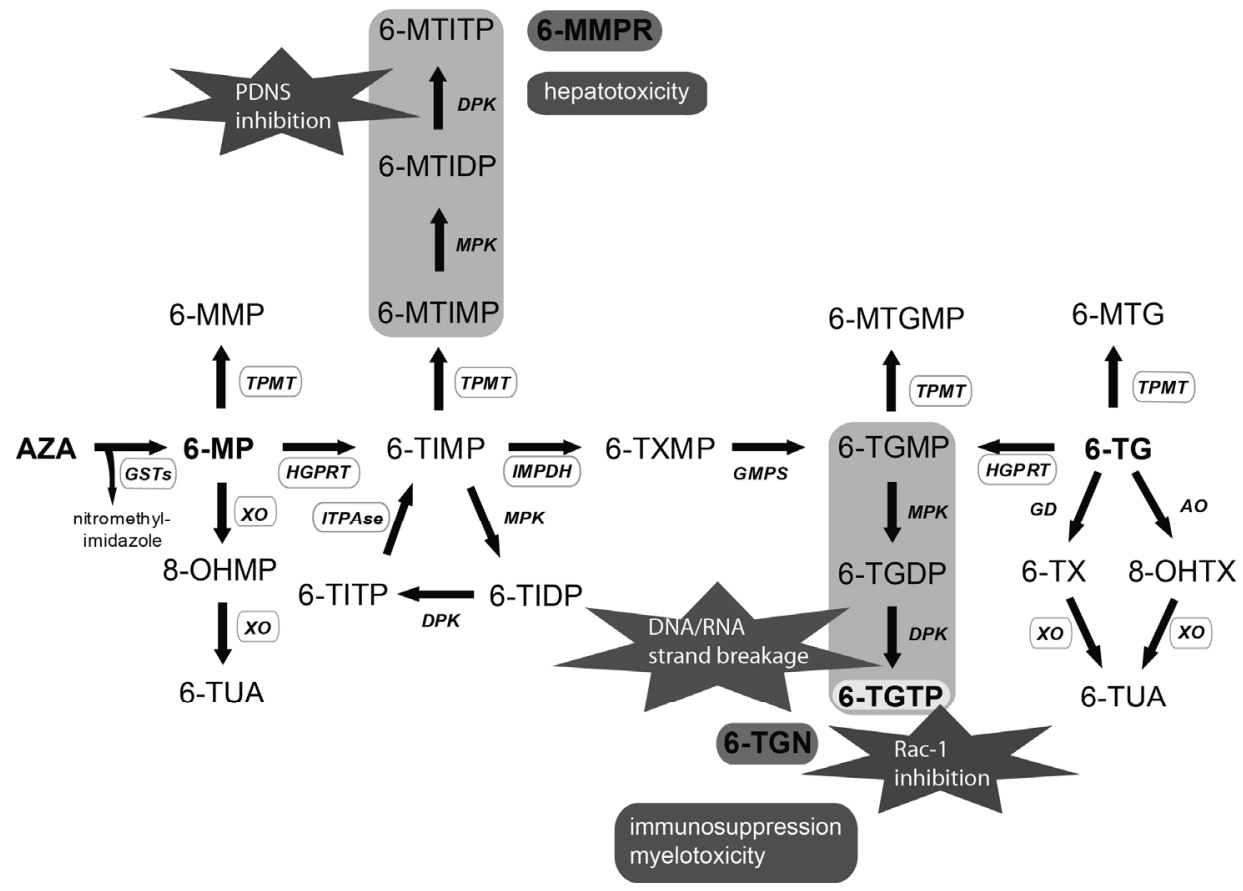

Figure 2.1 Thiopurine metabolism. AZA, azathioprine; 6-MP, 6-mercaptopurine; 6-MMP, 6-methylmercaptopurine; 8-OHMP, 8-hydroxy-6-mercaptopurine; 6-TUA, 6-thiouric acid; 6-MTIMP, 6-methylthioinosine monophosphate; 6-MTIDP, 6-methylthioinosine diphosphate; 6-MTITP, 6-methylthioinosine triphosphate; 6-TIMP, 6-thioinosine monophosphate; 6-TIDP, 6-thioinosine diphosphate; 6-TITP, 6-thioinosine triphosphate; 6-TXMP, 6-thioxanthosine monophosphate; 6-TGMP, 6-thioguanine monophosphate; 6-TGDP, 6-thioguanine diphosphate; 6-TGTP, 6-thioguanine triphosphate; 6-MTGMP, 6-methylthioguanine monophosphate; 6-TG, 6-thioguanine; 6-MTG, 6-methylthioguanine; XO, xanthine oxidase; TPMT, thiopurine S-methyl transferase; HGPRT, hypoxanthine-guanine phosphoribosyl transferase; IMPDH, inosine monophosphate dehydrogenase; GMPS, guanosine monophosphate synthetase; MPK, monophosphate kinase; DPK, diphosphate kinase; ITPase, inosine triphosphate pyrophosphatase; 6-TX, 6-thioxanthine; 8-OHTG, 8-hydroxy-6-thioguanine; GD, guanine deaminase; $\mathrm{AO}$, aldehyde oxidase. PDNS, purine de novo synthesis; DNA, deoxyribonucleic acid; RNA, ribonucleic acid. 6-MTIMP, 6-MTIDP and 6MTITP together form the 6-methylmercaptopurine ribonucleotides (6-MMPR). 6-TGMP, 6-TGDP and 6-TGTP together form the 6-thioguaninenucleotides (6-TGN). Enzymes encoded by genes that are subject to known genetic polymorphisms are encircled in grey. 
In recent years, the molecular mechanisms of action of the thiopurines have been elucidated at least partially. The 6-TGN are incorporated into DNA of leucocytes as fraudulent bases as a result of their structural similarity to the endogenous purine-base guanine, resulting in strand breakage and subsequently immunosuppression. ${ }^{6,10}$ However, toxic levels of 6-TGN may lead to (life-threatening) myelosuppression. ${ }^{11}$ One of the 6-TGN in particular, 6-TGTP, is considered to contribute to the immunosuppressive effects due to inhibition of Rac1 upon CD28 co-stimulation, inducing T-cell apoptosis. ${ }^{12}$ Rac1 is a small GTPase and plays a role in inhibiting T-cell apoptosis. 6-TGTP binds to Rac1 instead of GTP, thereby suppressing the activation of Rac1 target genes like mitogen-activated protein kinase, NF-KB, and bcl- $\mathrm{x}_{\mathrm{L}}$. Also, the 6-MMPR contribute to the antiproliferative properties of the thiopurines, probably through inhibition of purine de novo synthesis, but toxic 6-MMPR levels are associated with liver damage or myelotoxicity. ${ }^{11,13,14}$ Furthermore, AZA has an immunosuppressive effect additional to 6-MP alone and it is postulated that this is associated with an effect of the methylnitroimidazolyl substitute by a currently unknown mechanism. ${ }^{6}$ In addition, other mechanisms may contribute to the immunosuppressive effects of the thiopurines, such as interference with function of similar endogenous molecules like ATP and GTP, which play an important role as carriers of energy and cellular second messengers. ${ }^{15}$ In case of 6-TG, the cytotoxic potential of 6-MTG metabolites has previously been shown by inhibition of the first step of purine de novo synthesis. These metabolites may contribute to the immunosuppressive effect of thiopurines, although an association between 6-MTG levels and efficacy could not be shown in a recent study. ${ }^{16}$.

As mentioned before, the observed interindividual differences in therapeutic response or toxicity are partly explained by the variable formation of various active metabolites described above. This variation is at least partly caused by genetic polymorphisms of genes encoding for crucial enzymes in thiopurine metabolism. This in depth review focuses on this specific subject. For each enzyme its endogenous function and/or its role in thiopurine metabolism is discussed, as well as known polymorphisms, their prevalence, pharmacological consequences (e.g. resulting enzyme activity) of polymorphisms, conclusions regarding therapeutic consequences in IBD management and future research.

\section{Glutathione S-tranferases (GSTs)}

The pharmacological action of AZA is based on the release of 6-MP. This conversion has always been considered solely to be a non-enzymatic reaction, facilitated by glutathione or other endogenous sulphydryl-containing proteins. ${ }^{17,18}$ Recently however, Eklund and colleagues have demonstrated that this conversion is primarily catalysed by detoxifying glutathione S-tranferases (GSTs): they estimated the uncatalysed reaction of AZA with glutathione to be less than $1 \%$ of the GST-catalysed biotransformation. ${ }^{19}$ Of 
the 14 GSTs tested in their study, GSTs from the $\alpha$-class, A1-1 and A2-2, and from the $\mu$ class, $M 1-1$, showed the highest activity. ${ }^{19}$ These enzymes, all abundantly expressed in the human liver, mediate the release of 6-MP from AZA by the nucleophilic substitution involving glutathione. ${ }^{19}$ GST M1-1 is polymorphic and about $50 \%$ of the population lacks the required enzyme activity, which was shown to be caused by the absence of the corresponding gene on human chromosome $1 .{ }^{20}$ On the other hand, a duplicated GST M1-1 gene, that causes ultra rapid enzyme activity, has also been described. ${ }^{21}$ Furthermore, allelic variants in the $\alpha$-class have been characterised and both GSTS A1-1 and A2-2 show variable expression levels in human subjects implicating significant differences in the rate of 6-MP release from AZA. ${ }^{19}$

In contrast to 6-MP, an important aspect of AZA metabolism is the possible depletion of glutathione in hepatocytes, which eventually leads to mitochondrial injury and consequent ATP depletion. In isolated hepatocytes, the loss of ATP leads to cell necrosis rather than apoptosis, which requires ATP. The authors therefore conclude that a high hepatic GST activity in combination with a high dose of AZA can lead to glutathione depletion and cause necrotic liver lesions. ${ }^{19}$ Stocco and colleagues have recently reported that IBD patients with a high frequent GST-M1 genotype are at increased risk of developing adverse reactions during AZA treatment, including pancreatitis, bone marrow suppression, liver toxicity, arthralgia and neuropathy. Furthermore, among patients without adverse reactions there was a significant underrepresentation of the GST-M1 low frequent genotype among patients who developed lymphopenia. ${ }^{22}$

In conclusion, patients with non-functional alleles encoding for GSTs and lower enzyme activity will have slower rates of 6-MP release from AZA and will possibly experience less efficacy. On the other hand, patients with duplicated alleles for GSTs, and subsequently ultra rapid enzyme activity, probably have a predisposition for adverse reactions to AZA treatment, both due to promotion of excessively high concentrations of free 6-MP and its active metabolites, and depletion of cellular glutathione. ${ }^{19}$ Further prospective studies should be performed to confirm and elucidate these findings and to assess the usefulness of prior-to-treatment genotyping of these GST genes to prevent AZA related adverse reactions in patients with IBD.

\section{Xanthine oxidase (XO)}

$\mathrm{XO}$ is a cytoplasmic enzyme involved in the oxidation of endogenous substrates such as purines and pyrimidines, but also the exogenous thiopurines. Its activity is particularly high in the intestinal mucosa and liver, resulting in a substantial reduction of 6-MP bioavailability and subsequently lower levels of the active $6-\mathrm{TGN}^{23}$ XO catalyses the catabolism of 6-MP, first to 8-OHMP and subsequently to 6-TUA. ${ }^{24}$ Furthermore, recent data indicate that $\mathrm{XO}$ plays an important role in inflammatory diseases and various forms of tissue and vascular injuries. ${ }^{25}$ In kidney epithelial cells increased $X O$ gene expression has been reported following exposure to a number of inflammatory 
cytokines, including tumor necrosis factor- $\alpha$ (TNF $\alpha$ ), interferon $\gamma$, interleukin-1 (IL-1) and interleuking- 6 (IL-6). The effect of TNF $\alpha$, IL-1 and IL- 6 on XO gene activation may be tissue and/or cell specific. It has been suggested that the increased expression of XO may play a immunomodulatory and anti-viral role elicited by these cytokines. ${ }^{26}$ In patients with inflammatory bowel disease the inflammatory cytokines IL-1, IL-6 and TNF $\alpha$, are important mediators produced by activated macrophages, which target a broad variety of other types of cells. ${ }^{27}$

A 4-fold interindividual variation in hepatic XO activity exists and the presence of a subgroup of patients with low activity is suspected. On the other hand, there is also circumstantial evidence for extremely high XO activity in a small subgroup of patients treated with thiopurines. ${ }^{28}$ Gender related differences in XO activity are still unclear: Guerciolini and colleagues reported an approximately $20 \%$ higher enzyme activity in men than in women, ${ }^{29}$ but Relling and colleagues found a higher XO activity in females than in males. ${ }^{30}$ Moreover, XO exhibits higher activity in white subjects than in black subjects. $^{30}$

Interindividual differences in XO activity could be either genetically determined or influenced by environmental factors. ${ }^{26,29,31,32}$ Environmental influence was demonstrated clearly in Ethiopian cohorts, in whom XO activity is known to be polymorphic: Ethiopians living in Ethiopia showed higher $\mathrm{XO}$ activity than those living in Sweden. Neither smoking, nor gender related difference altered XO activity, indicating influence of other environmental factors on XO activity, such as dietary habits and inflammatory diseases. ${ }^{32}$

Competitive inhibition of $\mathrm{XO}$ with allopurinol (100 $\mathrm{mg} 3 \mathrm{dd}$ ), an analogue of hypoxanthine, results in an approximately 5 -fold increase of the bioavailability of oral 6-MP. ${ }^{24}$ Raised erythrocyte 6-TGN levels have been demonstrated in patients receiving allopurinol and dangerous myelotoxicity resulting from concurrent use of allopurinol and thiopurines has been reported. ${ }^{33,34}$ Interestingly, oxidised thiopurine metabolites may inhibit TPMT, resulting in even higher cytotoxic 6-TGN levels. ${ }^{35}$ For concurrent use of allopurinol and thiopurines a dose-reduction to $25-33 \%$ of standard daily dosages of AZA or 6-MP is recommended to prevent serious myelotoxicity. ${ }^{34}$ Generally, about twothirds of a normal dose appears to be inactivated by the catabolic pathway of XO.

Until recently, the molecular basis of the interindividual differences had been unclear. However, Kudo and colleagues have investigated the genetic variations in $\mathrm{XO}$ in a Japanese population. They have demonstrated that three single nucleotide polymorphisms (G514A, A3326C and A3662G) in the XO gene are involved in individual variations in $\mathrm{XO}$ activity, dividing the population in $\mathrm{XO}$-deficient subjects and subjects with low, normal and high activity respectively. ${ }^{36}$ It is unclear whether these pharmacogenetic data can easily be extrapolated to Caucasian and/or other populations. Polymorphisms in the gene that encodes the XO enzyme have scarcely been studied in IBD patients. Recently, Hawwa and colleagues studied the influence of two single nucleotide polymorphisms (A1936G and A2107G) in the XO gene on toxicity, haematological parameters and thiopurine metabolite levels in patients with acute 
lymphoblastic leukaemia (ALL) or IBD. Of the 35 IBD patients, three were heterozygous for both XO alleles, while only one of 19 ALL patients was heterozygous with both XO alleles. In the IBD patients there was a trend toward higher erythrocyte 6-MP concentrations in patients having low frequent XO alleles $(p=0.061)$. In IBD patients, myelotoxicity was found in three patients, with low frequent $\mathrm{XO}$ alleles present in one patient. Furthermore, there was no association found between low frequent XO alleles and any of the haematological parameters. Due to the small number of patients with myelotoxicity it was not possible to draw any conclusions. ${ }^{37}$

More studies of larger populations are needed to evaluate the influence of $X O$ polymorphisms on clinical outcome of thiopurine treatment of IBD. Until then it seems reasonable to assume adverse effects due to thiopurines or their active metabolites are increased in poor XO metabolisers, particularly in XO deficient patients. On the other hand, rapid $X O$ metabolisers may fail to respond thiopurine therapy due to low active metabolite formation.

\section{Thiopurine S-methyltransferase (TPMT)}

Amongst the most extensively studied genetic polymorphisms are those of TPMT, though neither its biological function nor its endogenous substrates are known. ${ }^{38}$ In AZA and 6-MP treated patients TPMT determines the delicate balance between the pharmacologically active 6-MMPR and the 6-TGN. 6-TG as such is less affected by TPMT. $^{39}$ In the 1980s, the genetic basis for TPMT deficiency was initially identified by Weinshilboum and colleagues. ${ }^{40}$ The $27-\mathrm{kb}$ gene encoding for TPMT is located on human chromosome 6 (6p22.3) and contains 10 exons. To date, 3 alleles associated with normal TPMT activity (TPMT*1 and 2 silent polymorphisms) and at least 27 alleles responsible for TPMT deficiency have been described $(T P M T * 2, * 3 A, * 3 B, * 3 C, * 3 D, * 4$, *5, *6, *7, *8, *9, *10, *11, *12,*13,*14, *15, *16, *17, *18, *19, *20,*21,*22, *23, $* 24, * 25){ }^{41-46}$ These alleles are characterised by one or more single nucleotide polymorphisms (SNPs) in the open reading frame (ORF) sequences of the TPMT gene. Moreover, several intronic mutations and mutations outside the ORF exist. A variable number tandem repeat (VNTR) within the TPMT promoter region of the TPMT gene has also been reported to modulate levels of TPMT activity, although its significance is controversial. $^{8,47,48}$ Enhanced degradation of TPMT proteins encoded by certain low frequent alleles has been proposed as the mechanism for lower TPMT protein and catalytic activity: whereas the degradation half-life of TPMT*1 is 18 hours, TPMT*2 and $T_{P M T}{ }^{*} 3 A$ have a strongly decreased half-life of 15 minutes. ${ }^{49}$ As a result of several SNPs in the TPMT-gene, the tertiary structure of the TPMT-protein is changed, leading to instability and decreased catalytic activity. ${ }^{8}$ On the other hand, ultra-high TPMT activity exists in $1-2 \%$ of the population. Very recently, the molecular basis for this phenomenon was elucidated in two patients from two testing centres exhibiting the highest TPMT activity: a polymorphism was characterised within a trinucleotide (GCC) 
repeat element of the TPMT promoter. Whereas 50 IBD patients with normal TPMT activity were all homozygous for $6 \mathrm{GCC}$ repeats $\left[(\mathrm{GCC})_{6}\right]$, these two patients were heterozygous for a variant allele carrying $7\left[(\mathrm{GCC})_{7}\right]$ and $5 \mathrm{GCC}$ repeats $\left[(\mathrm{GCC})_{5}\right]$ respectively, suggesting that alteration in the number of trinucleotide repeats is responsible for the ultra-high TPMT activity observed in these patients. ${ }^{50}$

The distribution of TPMT low frequent alleles differs significantly between ethnic populations. TPMT*3A (2.1-5.7\%) is the most frequently occurring low frequent allele in Caucasian populations, followed by TPMT*2 (0.2-0.7\%) and TPMT*3C (0.1-0.8\%) accounting for the vast majority (>95\%) of low frequent alleles., ${ }^{8,15,38,41,51-57}$ In Asians and African populations however, $T P M T^{*} 3 C(1.0-7.6 \%)$ is the most frequently occurring low frequent allele. ${ }^{8,58-60}$

There is high correlation between TPMT geno- and phenotype. ${ }^{42,61}$ In several studies focussing on TPMT geno-phenotype relationship, overall concordance rates from different population studies ranged from 76 to $99 \%$, though in case of intermediate metabolisers the correlation is much lower. These discrepancies could possibly be explained by the influence of genetic factors including regulatory elements of the TPMT gene or other loci as well as epigenetic factors, such as TPMT inhibition by concomitant drugs. ${ }^{8}$ The resulting frequency distribution of TPMT activity in Caucasian populations is trimodal: approximately $89 \%$ of the population is homozygous for the high frequent allele (homozygous $T P M T^{H}$ ) and consequently has a high enzyme activity $(13.50 \pm 1.86 \mathrm{U} / \mathrm{ml} \mathrm{RBC}), 11 \%$ inherits one high frequent allele and one low frequent allele (heterozygous $T P M T^{H} / T P M T^{L}$ ) and has intermediate levels of enzyme activity (7.20 $\pm 1.08 \mathrm{U} / \mathrm{ml} \mathrm{RBC)}$, while 1 in 300 subjects has 2 low frequent alleles (homozygous $T_{P M T^{L}}$ ) and low or no detectable enzyme activity at all. ${ }^{15,40}$ TPMT activity is high in children compared to adults and among adults higher in men than in women and higher in smokers than in non-smokers. ${ }^{42,62,63}$ Also, TPMT activity significantly increases during thiopurine treatment as a result of enzyme induction. ${ }^{6}$ However, in a doubleblind randomised prospective trial in $C D$ patients treated with a fixed oral dose of either AZA (2-2,5 mg/kg/day) or mesalazine ( $4 \mathrm{~g} /$ day) during a one year postoperative period, neither mesalazine nor AZA significantly affected TPMT activity during the study period. $^{64}$

Reliable polymerase chain reaction (PCR)-based methods have been developed for detecting the major inactivating SNPs at the human TPMT locus. ${ }^{38,53,65}$ In brief, leukocyte DNA is amplified by PCR techniques and digested by specific restriction enzymes. The resulting DNA-fragments are analyzed by gel electrophoresis. Finally, the identified SNPs yield a specific TPMT genotype (Figure 2.2). Alternatively, TPMT phenotype can be determined by measuring TPMT activity based on the in vitro conversion of 6-MP to 6-MMP. ${ }^{66}$ Phenotyping may be more relevant and provide more information in some specific cases, since a large variation in TPMT activity exists between individuals with the same genotype. However, as mentioned before, phenotype can alter due to thiopurine therapy and the use of concomitant medication, 
whereas genotype can not. And, phenotyping may sometimes lead to misclassification of a patient's TPMT status, for example after a blood transfusion. ${ }^{67}$

The use of determining TPMT status, the exact moment when for geno- or phenotyping and the clinical implications are currently subject for debate. ${ }^{15,68}$ Patients on normal therapeutic thiopurine dose regimens with one or two low frequent alleles have less TPMT activity and consequently grossly elevated 6-TGN concentrations, increasing the risk of the development of bone marrow suppression. ${ }^{15}$ Conversely, some patients with very high TPMT activity, so called ultra-methylators, develop suboptimal 6-TGN concentrations by shunting 6-MP away to 6-MMP resulting in treatment failure. ${ }^{69}$ In a retrospective study with 106 IBD patients, TPMT genotype predicted phenotype which correlated to drug efficacy and toxicity: intermediate TPMT activity was associated with an increased risk of AZA toxicity (OR: 5.4 ), while high activity (>14 U/ml RBC) predicted treatment failure (OR: 0.21). ${ }^{55}$ In a prospective study with 67 patients with rheumatic disease, 6 were heterozygous for low frequent TPMT alleles, 5 of whom discontinued therapy within the first month of AZA treatment due to reduced total leukocyte counts. ${ }^{70}$ In another study with 262 IBD patients, TPMT genotype predicted leukopenia with an OR of 6.32 for patients with low frequent TPMT alleles compared to patients with high frequent alleles. ${ }^{71}$ In another study with 113 IBD patients, it was demonstrated that during the initial four months, low TPMT activity correlated with low neutrophil counts. Also, patients with low TPMT activity could be safely managed on a low $\left(<2.0 \mathrm{mg} / \mathrm{kg}\right.$ ) AZA dose. ${ }^{72}$ Similar results were reported in a cohort of 71 patients with CD: patients with normal TPMT status received AZA in an initial dose of $2-2.5 \mathrm{mg} / \mathrm{kg} /$ day compared to $1-1.5 \mathrm{mg} / \mathrm{kg} /$ day for patients with intermediate enzyme activity and none developed acute leukopenia. ${ }^{73}$ In general, a 50\% starting dose, that is $1-1.5 \mathrm{mg} / \mathrm{kg} /$ day $A Z A$ or $0.75 \mathrm{mg} / \mathrm{kg} /$ day $6-\mathrm{MP}$, is advocated in patients with heterozygous TPMT alleles. ${ }^{74}$ Some recommend an even safer initial dose reduction to $33 \%$ of the standard dose in heterozygotes. ${ }^{75}$ It is generally recommended that patients homozygous for low frequent alleles should not receive thiopurines at all for IBD or a significantly reduced dose: $0.16-0.29 \mathrm{mg} / \mathrm{kg}$ AZA daily which is approximately $10 \%$ of standard dose. ${ }^{74-76}$ Recently, in a case-report it was demonstrated that even $5 \%$ of the standard AZA dose can be dangerous in patients homozygous for low frequent alleles and may cause severe leukopenia. Therefore, it was advocated by the authors that this rare group of patients should be treated with greatest caution and very frequent monitoring. ${ }^{77}$

In several other studies no or very poor correlation has been found between TPMT status and thiopurine toxicity. In a study with 41 leukopenic patients with Crohn's disease (CD) treated with AZA, only $27 \%$ of the patients had one or two low frequent TPMT alleles. ${ }^{78}$ Myelosuppression was more often caused by other factors, such as viral infections (cytomegalovirus, varicella and parvovirus B19), drugs interfering with AZA metabolism (allopurinol and 5-aminosalicylates) or drugs, which cause bone marrow suppression themselves (trimethoprim-sulfamethoxazole, captopril and metronidazole). In the majority of cases however, there was no obvious cause of 
myelosuppression. The lag time to the first appearance of myelosuppression was longer in patients with high frequent alleles, though. Similar results were found in another study with 56 IBD patients: patients with adverse reactions to AZA or 6-MP were reported to have more low frequent alleles, but this was not statistically significant. ${ }^{79}$ Therefore, it was concluded that TPMT genotype does not predict adverse reactions to thiopurines in this population.

Recently, several pharmacoeconomic evaluations of TPMT geno- or phenotyping prior to thiopurine therapy of IBD have been published. Priest and colleagues developed a pharmacoeconomic model to compare treatment costs and effects (quality adjusted life years = QALYS) of AZA compared to other therapies in theoretical populations of 1000 IBD patients over a 1 year period. They presented beneficial results for AZA therapy in general and specially for prospective pharmacogenetic testing: net cost savings of approximately $\$ 1,000,000$ (converted NZD 2,500,000) were realised for AZA versus no immunosuppressive therapy, AZA generated 877 QALYs and TPMT geno- or phenotyping generated net cost savings of approximately $\$ 63,000$ (converted NZD $120,000)$ compared to no prior testing. Furthermore, they concluded TPMT phenotyping is likely to be more cost effective than genotyping because of lower assay costs and greater probability of pre-empting neutropenia. ${ }^{80}$ In another pharmacoeconomic study in IBD patients, after analysis of literature to calculate the expected frequency of leukopenia and its relationship with TPMT polymorphism, a decision analysis was applied to assess the cost of a pre-treatment genotyping strategy, taking into account direct costs and cost per life-year saved: this resulted in pre-

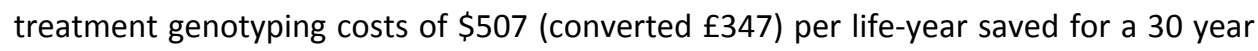

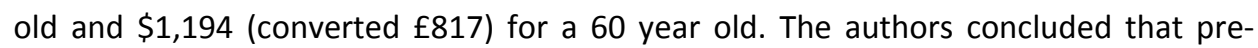
treatment screening for TPMT polymorphisms in IBD patients commencing AZA therapy represented good value for money. ${ }^{81}$ Others have yet to be convinced TPMT genotyping is the most cost-effective method to improve outcome in the management of AZA-associated leukopenia in IBD-patients; they stress the pharmacoeconomic models should be tested in medical practice and recommend the much cheaper frequent full blood counts for now. ${ }^{82}$ In fact, this strategy was prospectively tested in practice by Sayani and colleagues in a cohort of 29 IBD-patients: testing of TPMT enzyme activity before initiating AZA therapy incurred additional costs and did not predict AZA-induced toxicity. ${ }^{83}$ However, this study was probably underpowered as only one in 300 patients is a poor metaboliser and most likely to benefit from pre-treatment pharmacogenetic testing. Therefore, two large prospective trials are currently being undertaken in the UK (TARGET study) and the Netherlands (TOPIC study). In these studies, IBD patients will be randomised in two groups: one group will receive standard care, while the other group will undergo pre-treatment TPMT genotyping followed by a personalised AZA or 6-MP dosing advice. The primary objectives in these studies are (A) an analysis of (haematological) adverse drug reactions associated with thiopurine use of patients in the intervention (genotyping) and control (standard care) arm, (B) an analysis of the clinical outcome in both patient groups, (C) an analysis of quality of life 
in both patient groups, (D) an assessment of costs associated with resource use and treatment costs in both patient groups and (E) a cost-effectiveness analysis of TPMT genotyping in patients receiving thiopurines for immunosuppression using the data collected from objective (A) to (D). ${ }^{84}$

In conclusion, whereas TPMT genotyping seems to play a role in the prediction of early drug toxicity in patients who have not previously been exposed to AZA or 6-MP, the predictive value for drug toxicity in patients on thiopurines may be limited. ${ }^{15}$ Studies with larger numbers of patients are needed to confirm this hypothesis. If TPMT genotyping is available, it is appropriate to genotype an IBD-patient before initiating thiopurine treatment. Homozygous low frequent TPMT allele carriers should carefully receive $10 \%$ of standard starting dose or no thiopurine at all, whereas heterozygous patients should receive $50 \%$ of standard starting dose and homozygous high frequent TPMT allele carriers can be safely given standard starting dose. The results from pharmacoeconomic models favour pre-treatment testing of TPMT polymorphisms in IBD patients, in pharmacoeconomic trials that are currently being undertaken, this strategy will ultimately be tested in a prospective way.

\section{Inosine triphosphate pyrophosphatase (ITPase)}

ITPase catalyzes the pyrophosphohydrolysis of inosine triphosphate (ITP) to inosine monophosphate (IMP) and ITPase deficiency leads to abnormal accumulation of ITP, which is not known to be associated with any defined pathology. ${ }^{85}$ In ITPase deficient patients treated with AZA or 6-MP, accumulation of 6-TITP is suggested. ${ }^{86}$ The gene encoding ITPase, ITPA, is located on chromosome 20 and 5 SNPs have been identified so far, of which 3 (G138A, G561A, G708A) are silent and 2 (C94A, IVS2 + A21C) are associated with decreased ITPase activity. ${ }^{87} \mathrm{C} 94 \mathrm{~A}$ is the most frequently occurring SNP. Homozygotes for the C94A missense mutation have no enzyme activity at all and IVS2+A21C homozygotes have approximately $60 \%$ of normal ITPase activity, but the exact prevalence is unknown. It is estimated that in Caucasians the C94A allele frequency is approximately $6 \%$ and heterozygotes possess $22.5 \%$ of normal enzyme activity, whereas approximately $13 \%$ of the population are IVS2 $+A 21 C$ heterozygotes possessing $60 \%$ of normal enzyme activity. Also, C94A/IVS2+A21C compound heterozygotes have been identified, possessing $10 \%$ of ITPase activity. ${ }^{87}$ In a Japanese cohort of 100 healthy individuals, C94A allele frequency was $13 \%$, whereas IVS2+A21C polymorphism was not found. ${ }^{88}$

The identification of SNPs in ITPA is done in a similar way as TPMT genotyping, obviously with different primers and restriction enzymes. ${ }^{87}$ ITPase-activity can be determined by measuring the in vitro conversion of ITP to IMP with a HPLC method. ${ }^{89}$ Recently, in a study with 62 IBD patients, the association between polymorphism in the ITPA gene and adverse drug reactions to AZA therapy has been investigated: the C94A ITPase deficiency-associated allele significantly correlated with adverse reactions (odds 
ratio 4.2 ), such as flu-like symptoms, rash and pancreatitis. ${ }^{86}$ Moreover, in the same study no correlation was found between these adverse reactions and TPMT genotype. In another study by Zelinkova and colleagues the C94A allele was demonstrated to predict leukopenia with an OR of 3.50 for patients with low frequent compared to patients with high frequent ITP alleles. The only patient with homozygous C94A low frequent alleles however did not develop leukopenia, weakening the relationship between ITPA genotype and the occurrence of adverse reactions. ${ }^{71}$

However, these results were contradicted by two other studies with 73 and 41 IBD patients, respectively, in which no significant association between ITPA genotype C94A polymorphism and adverse effects to thiopurine drugs could be demonstrated. ${ }^{90,91}$ There is little experience with ITPA genotyping in IBD populations and its predictive value. More and larger studies are necessary to confirm and clarify these provisional data.

\section{Hypoxanthine phosphoribosyltransferase (HGPRT)}

Hypoxanthine phosphoribosyltransferase carries out the first anabolic step in thiopurine metabolism to produce 6-TIMP from 6-MP. Only limited information exists on the range of HGPRT activity. However, we know HGPRT is completely deficient in subjects with the very rare $(1: 380,000)$ Lesch-Nyhan syndrome, which is characterised by an overproduction of uric acid, self-mutilating behaviour such as lip biting and head banging, delayed developmental milestones, mental retardation and extrapyramidal and pyramidal motor symptoms. ${ }^{6,92,93}$ Thiopurines are not cytotoxic in Lesch-Nyhan patients and SNPs in the HGPRT gene appear to be the molecular basis of this syndrome. ${ }^{94}$ At least 2,000 SNPs throughout the HGPRT gene coding region from exon 1-9 have been reported. ${ }^{95}$ HGPRT enzyme deficiency presents with a wide range of neurological symptoms. The overall severity of the disease is associated with mutations permitting some degree of residual enzyme activity. ${ }^{96}$ HGPRT is partially deficient in people with the Kelley-Seegmiller syndrome, and can also give rise to a wide range of neurological symptoms and renal insufficiency. ${ }^{97}$ Moreover, HGPRT activity increases during long-term thiopurine treatment. ${ }^{6}$

In conclusion, in patients with partial or complete HGPRT deficiency, thiopurines will be less or not effective, because the pharmacologically active metabolites are formed to a lesser extent if at all. Screening for HGPRT deficiency is of minor significance as these patients will probably be identified at an early stage due to their specific array of clinical symptoms. 


\section{Inosine monophosphate dehydrogenase (IMPDH)}

Inosine monophosphate dehydrogenase transforms 6-TIMP into 6-TXMP. In contrast to 6-TG, AZA and 6-MP need the enzyme IMPDH to ultimately form the pharmacologically active 6-TGN. AZA or 6-MP resistance may be caused by very high TPMT activity (in socalled ultra-methylators) or XO activity on one hand, but may also be the result from dysfunctional alleles of the IMPDH gene on the other (also see Figure 2.1). IMPDH is a strategically positioned enzyme in thiopurine metabolism and exists in human as two isoforms, type I and II, which have comparable catalytic activity and substrate affinity and show $84 \%$ resemblance in peptide sequences. However, differences in the regulation and expression of the two isoforms exist: there is more variation in the level and pattern of IMPDH type I than described for the type II isoform. This variation in IMPDH type I expression is mainly caused by the existence of 3 promotors (P1, P2 and P3) within IMPDH1, of which in most tissues P3 is the major promoter. ${ }^{98-101}$

In a small cohort of 20 AZA resistant IBD patients with normal TPMT activity, sequence variations in the two genes encoding for IMPDH (IMPDH1 and IMPDH2) were screened for: a 9 base pair insertion in the IMPDH1 P3 promotor was found in one of the patients with severe AZA resistance. The authors concluded that the absence of functional SNPS in the other 19 patients shows that if IMPDH genetic variability contributes to AZA resistance at all, it does so infrequently. ${ }^{101}$ Recently, in another study with 50 IBD patients on stable thiopurine therapy, IMPDH activity as well as thiopurine metabolite concentrations were measured. A negative correlation between IMPDH activity and 6-MMPR levels was shown, but no evident correlation with 6-TGN levels or 6-MMPR/ 6-TGN ratio was demonstrated. The authors therefore concluded that the role of IMPDH in balancing the formation of methylated (6-MMPR) and phosphorylated metabolites (6-TGN) was not evident. ${ }^{102}$

In conclusion, although IMPDH is an essential enzyme in the formation of pharmacologically active metabolites of AZA or 6-MP, data from two small studies do not support geno- or phenotyping this enzyme as a correlation with SNPs or enzyme activity is lacking. These findings are probably explained by the very low prevalence of IMPDH modifications. In individual cases of severe AZA or 6-MP resistance without treatment alternatives, the determination of IMPDH geno- or phenotype may be valuable.

\section{Multidrug resistance (MDR) proteins}

P-glycoprotein (P-gp) is an ATP-dependent transmembrane efflux pump, which can extrude a range of structurally diverse toxic xenobiotic compounds from cells. An important function of these proteins is to protect organisms against many of the toxic xenobiotics, which they can naturally be exposed to. Furthermore, P-gp in the bloodbrain barrier and other blood-tissue barriers protects sensitive organs from exposure to 
toxic compounds that may have entered the bloodstream. ${ }^{103}$ These efflux pumps serve as a defence mechanism and determine bioavailability and central nervous system concentrations of many drugs. P-gp exhibits high expression levels in the intestinal wall and in proximal tubular cells of the kidney cells, reflecting its role on drug pharmacokinetics. ${ }^{104}$ Cells overproducing drug-transporting proteins may become resistant to a broad spectrum of drugs with different structures or cellular targets, resulting in so-called multidrug resistance (MDR). P-gp is encoded by the human $A B C B 1$ gene, better known as multidrug resistance 1 (MDR1) gene. ${ }^{103}$ Genetic variations of human MDR1 gene have been studied extensively and to date at least 50 SNPs and 3 insertion/deletion polymorphisms have been reported. ${ }^{105}$ Moreover, MDR1 is an attractive candidate gene for the pathogenesis of IBD. The physiological importance of $\mathrm{P}$-gp in the gastrointestinal tract has been demonstrated with the MDR1 knock-out mice model, where spontaneous colitis develops in a specific pathogen-free environment. $^{105}$

Many of the drugs used in IBD are known substrates for P-gp, such as corticosteroids, cyclosporine, tacrolimus and methotrexate. ${ }^{106-109}$ Recently, P-gp has also been held responsible for resistance to the most commonly used immunosuppressants in IBD, the thiopurines, even though these agents usually are not considered to be substrates for P-gp. ${ }^{110}$ In this study, it was demonstrated that human MDR1 transfected murine L1210/VMDRC.06 leukemia cells express relatively large amounts of P-gp and high resistance to 6-MP and other purine and pyrimidine nucleobase and nucleoside analogues. $^{110}$ In another recent study by Mendoza and colleagues, MDR1 polymorphisms (G2677T/A, C3435T) have been correlated to response to AZA therapy in $76 \mathrm{CD}$ patients: higher frequencies of $2677 T T$ and $3435 T T$ genotypes and $2677 T / 3435 T$ haplotype were found in patients unresponsive to AZA. ${ }^{104}$ Therefore, the hypothesis that failure to thiopurines in CD could be at least partially related to high P-gp expression due to MDR1 polymorphisms in intestinal mucosa and lymphocytes seems reasonable. The authors suggest additional replications in independent populations with higher statistical power could confirm the real impact of these polymorphisms as genetic markers involved in AZA response. ${ }^{104}$

Another class of ATP-dependent transmembrane drug transporters are the multidrug resistance proteins (MRPs or ABCCs), which can not only cause cellular multidrug resistance in vitro, but are also likely to contribute to the intrinsic or acquired resistance against anticancer drugs used in patients. ${ }^{111}$ To date at least thirteen members of the MRP family are known. MRPs are organic anion transporters, but also have the ability to transport nucleoside monophosphate analogues. ${ }^{112}$ Wijnhold and colleagues have previously reported on the functional characterisation of MRP5 (ABCC5); they have found resistance against 6-MP and 6-TG in cell lines over-expressing human MRP5. This resistance was due to an increased extrusion of the 6-TIMP, one of the precursors of the active 6-TGN and 6-MMPR (Figure 2.1), from cells overproducing MRP5. The authors speculated MRP5 may be involved in some cases of unexplained thiopurine resistance in ALL. ${ }^{111}$ More recently, Wielinga and colleagues have analysed 6-MP 
metabolites formed in human embryonic kidney cells and determined which metabolites are extruded by MRP4 (ABCC4) and MRP5 (ABCC5). They found that 6-TIMP is transported both by MRP4 and MRP5; MRP5 showed the highest transport rate. In contrast, only MRP5 transported 6-TXMP. The authors concluded that all major thiopurine monophosphates, important for the efficacy of 6-MP treatment, are transported by MRP4 en MRP5, although there are subtle differences in substrate specificity of the two transporters. ${ }^{113}$

It is plausible to assume that this intrinsic or acquired cellular drug-resistance may play an important role in patients with IBD, which may lead to a decreased immunosuppressive effect of thiopurine drugs in these patients. The question is whether MRP4 and MRP5 are expressed in leukocytes, the proposed target cells for thiopurines. Furthermore, since most MRP/ABCC transporters are subject to inhibition by a variety of compounds, it is interesting to study inhibitors of these transporters to modulate the efflux of thiopurine precursors. Mesalazine may be a good candidate for this purpose. ${ }^{114}$ More knowledge of specific substrates, inhibitors and inducers of the MRPs involved may therefore have important implications for improving thiopurine efficacy. Also, more knowledge of single nucleotide polymorphisms in the genes encoding for MRP4 and MRP5, which are known to be highly polymorphic, is required. These genetic polymorphisms in MRP-genes may result in a large variation in therapeutic response to thiopurines used in IBD.

\section{Discussion}

Thiopurine metabolism in human is complex. Many anabolic and catabolic enzymes play an important role in the formation of the pharmacologically active thiopurine metabolites. These crucial enzymes are mainly encoded by genes subject to genetic polymorphisms. This in depth review focuses on genetic variations in the encoding genes, the resulting enzyme activity and, ultimately, the therapeutic consequences in IBD management.

GSTs seem to play a prominent role in the conversion of AZA to 6-MP; whereas patients with non-functional genes will possibly experience less thiopurine efficacy, patients with duplicated alleles are predisposed to adverse reactions, because they develop high 6-MP levels. However, this pharmacogenetic variation can easily be circumvented by using 6-MP as such rather than AZA. In fact, 6-MP is the drug of choice for IBD in the US. In Europe though, AZA is the only registered thiopurine for this indication.

$\mathrm{XO}$ is probably the most important enzyme determining thiopurine bioavailability, which is $16-72 \%$ for AZA and $5-37 \%$ for $6-\mathrm{MP}$, and is responsible for these large interindividual variations in it. ${ }^{7}$ These interindividual differences in XO activity could be either genetically determined, but also caused by environmental factors as IBD itself. Furthermore, the molecular basis of interindividual differences in $\mathrm{XO}$ activity was very recently demonstrated in an Asian cohort only, and to date it is unclear whether these 
pharmacogenetic data can easily be extrapolated to Caucasian and/or other populations. Both these facts make the added value of standard pharmacogenetic screening prior to treatment for genes expressing $\mathrm{XO}$ in Caucasian populations questionable, at least for now.

Genetic polymorphisms of TPMT are one of the first and most extensively studied areas of pharmacogenetics. The most frequently occurring SNPs have been identified in several races, but rare SNPs are still being added to the list. Furthermore, we know that the overall concordance rates for TPMT geno-phenotype relationship are fairly good, the resulting frequency distribution of TPMT activity in Caucasians is trimodal and TPMT enzyme activity can be influenced by sex, age, smoking habits, thiopurine treatment and possibly concomitant medication. We also know for a fact that patients with homozygous low frequent alleles will develop toxic 6-TGN levels and severe leukopenia consequently, when treated with standard thiopurine dose, and patients with just one low frequent TPMT allele are at increased risk. On the other hand, one should realise that most cases of leukopenia still occur in the high frequent alleles baring population. Quite a few of studies have been conducted studying the predictive value of TPMT genotype in prevention of thiopurine toxicity and conflicting results have been published. Nevertheless, it seems plausible to assume the following, until studies have confirmed this hypothesis: whereas TPMT genotyping seems to be involved in the prediction of early thiopurine toxicity in IBD patients who have not been previously exposed to thiopurines, the predictive value for thiopurine toxicity in patients established on thiopurines may be limited. When TPMT genotype is known before thiopurine therapy, homozygous low frequent allele carriers should carefully receive $10 \%$ of standard starting dose or no thiopurine at all, heterozygotes should receive half of the standard starting dose and patients with high frequent alleles can be given the full starting dose. Subsequently, therapeutic drug monitoring (TDM) can be used to fine-tune thiopurine therapy by measuring thiopurine metabolite levels. ${ }^{11,115,116}$ With respect to cost-effectiveness, the results from pharmacoeconomic models favour pretreatment testing of TPMT polymorphisms in IBD patients. These results ultimately have to be confirmed in prospective pharmacoeconomic trials to convince those who bear the costs in national health care programs. In fact, these studies are currently performed.

Also for ITPase, trials have demonstrated conflicting pharmacogenetic results: the seemingly most important SNP, C94A, has been linked to various adverse reactions to AZA in some studies, while no significant association between ITPA genotype and thiopurine toxicity could be demonstrated in others. Therefore, for the present the predictive value is unclear and more and larger studies are needed to clarify these provisional data.

The gene encoding HGPRT is subject to genetic variation and numerous SNPs have been identified. Patients with Lesch-Nyhan syndrome, who are completely HGPRT deficient, will not benefit from treatment with thiopurines as the pharmacologically active 6-TGN cannot be formed. Patients with Kelley-Seegmiller syndrome have a partial HGPRT 
deficiency and will produce 6-TGN to a lesser extent when given thiopurines. Presumably, redundant thiopurine treatment of these patients can be prevented, because patients with both these syndromes will be diagnosed at an early stage as they will suffer from specific symptoms.

In addition to HGPRT, IMPDH is essential for the ultimate formation of the active 6-TGN. An infrequent genetic defect in the IMPDH gene has been held responsible for severe AZA resistance in one patient, but IMPDH activity did not correlate with 6-TGN levels in a small cohort of IBD patients. Therefore, the predictive value of IMPDH genoor phenotyping has been most uncertain up to now.

Last but not least, MDR polymorphisms have been correlated to response to AZA therapy in $C D$ patients and several genotypes and one haplotype with a predictive value for resistance to AZA have been identified. The hypothesis that failure to thiopurines in IBD could at least partly be related to high P-gp expression due to MDR1 polymorphisms seems plausible, but needs confirmation in larger cohorts of patients. Also, the influence of polymorphisms of genes encoding MRP4 and MRP5 on thiopurine metabolism and clinical response seems an important topic and needs further investigation in IBD patients.

Despite the ever-increasing knowledge on thiopurine metabolism, genetic polymorphisms of genes encoding for crucial enzymes and its consequences, limited practical information on the utilisation of pharmacogenetic knowledge in medical practice exists. Several conditions have to be fulfilled before this will be the case. The first and most obvious condition is knowledge of the gene mutation underlying the difference in drug response, and its exact location on the human chromosome. Furthermore, knowledge of the prevalence of the polymorphism of interest is important. This condition is fulfilled to a large extent for genes encoding for thiopurine metabolising enzymes as described above, but on the other hand knowledge in this field is ever growing. The second condition is that pharmacological consequences of baring certain low frequent alleles are clinically relevant. Although pharmacogenetics is scientifically interesting, it is not a goal in itself but an instrument to predict the clinical manifestations of a drug regarding efficacy or toxicity. There will only be a place for pharmacogenetics in medical practice when these predictions result in significant therapeutic benefits. This is definitely the case for thiopurine pharmacogenetics: a lifethreatening leukopenia can possibly be prevented by prior-to-treatment genotyping of crucial enzymes in thiopurine metabolism. Subsequently, this information should be translated into a practical advice or a particular action. Therefore, the third condition is the availability of evidence-based practical guidelines with information on how to act therapeutically (which drug in which dose) in patients with specific gene mutations. Although in the past two decades, knowledge about pharmacogenetics has been extended dramatically, the translation into practical guidelines is still scarce and it will take much effort to close this gap and to give tailor-made advice. Also for thiopurine pharmacogenetics this gap has to be closed, although the first examples of practical guidelines already exist. In the Netherlands, the Pharmacogenetics Working Party has 
developed national guidelines to moderate pharmacogenetic advice. ${ }^{117}$ These guidelines are available online for every Dutch pharmacist and contain practical advices for dosing thiopurines in IBD for most prevalent TPMT genotypes. Finally, the fourth condition is that pharmacogenetic techniques are available for genotyping; this is no issue of concern for thiopurine pharmacogenetics.

In conclusion, interindividual differences in therapeutic response or toxicity to thiopurines are to a large extent explained by the variable formation of active metabolites, which in turn is at least in part caused by genetic polymorphisms of the genes encoding crucial enzymes in thiopurine metabolism. Pharmacogenetic knowledge in this field has extended dramatically and is rapidly increasing still, but the translation into practical guidelines with tailor-made advices will take much effort in the years to come. 


\section{References}

1. Adler DJ, Korelitz BI. The therapeutic efficacy of 6-mercaptopurine in refractory ulcerative colitis. Am J Gastroenterol 1990;85:717-22.

2. Pearson DC, May GR, Fick GH, Sutherland LR. Azathioprine and 6-mercaptopurine in Crohn disease. A meta-analysis. Ann Intern Med 1995;123:132-42.

3. Sandborn WJ. A review of immune modifier therapy for inflammatory bowel disease: azathioprine, 6-mercaptopurine, cyclosporine, and methotrexate. Am J Gastroenterol 1996;91:423-33.

4. McGovern DP, Travis SP, Duley J, Shobowale-Bakre el M, Dalton HR. Azathioprine intolerance in patients with IBD may be imidazole-related and is independent of TPMT activity. Gastroenterology 2002;122:838-9.

5. Hindorf $U$, Lindqvist $M$, Hildebrand $H$, Fagerberg $U$, Almer S. Adverse events leading to modification of therapy in a large cohort of patients with inflammatory bowel disease. Aliment Pharmacol Ther 2006;24:331-42.

6. Lennard L. The clinical pharmacology of 6-mercaptopurine. Eur J Clin Pharmacol 1992;43:329-39.

7. Derijks LJ, Gilissen LP, Hooymans PM, Hommes DW. Review article: thiopurines in inflammatory bowel disease. Aliment Pharmacol Ther 2006;24:715-29.

8. Sahasranaman S, Howard D, Roy S. Clinical pharmacology and pharmacogenetics of thiopurines. Eur J Clin Pharmacol 2008;64:753-67.

9. Dubinsky MC, Hassard PV, Seidman EG, Kam LY, Abreu MT, Targan SR, Vasiliauskas EA. An open-label pilot study using thioguanine as a therapeutic alternative in Crohn's disease patients resistant to 6mercaptopurine therapy. Inflamm Bowel Dis 2001;7:181-9.

10. Fairchild CR, Maybaum J, Kennedy KA. Concurrent unilateral chromatid damage and DNA strand breakage in response to 6-thioguanine treatment. Biochem Pharmacol 1986;35:3533-41.

11. Dubinsky MC, Lamothe S, Yang HY, Targan SR, Sinnett D, Théorêt Y, Seidman EG. Pharmacogenomics and metabolite measurement for 6-mercaptopurine therapy in inflammatory bowel disease. Gastroenterology 2000;118:705-13.

12. Tiede I, Fritz G, Strand S, Poppe D, Dvorsky R, Strand D, Lehr HA, Wirtz S, Becker C, Atreya R, Mudter J, Hildner K, Bartsch B, Holtmann M, Blumberg R, Walczak H, Iven H, Galle PR, Ahmadian MR, Neurath MF. CD28-dependent Rac1 activation is the molecular target of azathioprine in primary human CD4+ T lymphocytes. J Clin Invest 2003;111:1133-45.

13. Dervieux T, Blanco JG, Krynetski EY, Vanin EF, Roussel MF, Relling MV. Differing contribution of thiopurine methyltransferase to mercaptopurine versus thioguanine effects in human leukemic cells. Cancer Res 2001;61:5810-6.

14. Gilissen LP, Derijks LJ, Verhoeven HM, Bierau J, Hooymans PM, Hommes DW, Engels LG. Pancytopenia due to high 6-methylmercaptopurine levels in a 6-mercaptopurine treated patient with Crohn's disease. Dig Liver Dis 2007;39:182-6.

15. Lennard L. TPMT in the treatment of Crohn's disease with azathioprine. Gut 2002;51:143-6.

16. Herrlinger KR, Fellermann K, Fischer C, Kreisel W, Deibert P, Schoelmerich J, Fleig WE, Ruhl A, Reinshagen M, Greinwald R, Stange EF, Schwab M. Thioguanine-nucleotides do not predict efficacy of tioguanine in Crohn's disease. Aliment Pharmacol Ther 2004;19:1269-76.

17. Schwab M, Klotz U. Pharmacokinetic considerations in the treatment of inflammatory bowel disease. Clin Pharmacokinet 2001;40:723-51.

18. Ardizzone S, Porro GB. Comparative tolerability of therapies for ulcerative colitis. Drug Saf 2002;25: 561-82.

19. Eklund $\mathrm{BI}$, Moberg M, Bergquist J, Mannervik B. Divergent activities of human glutathione transferases in the bioactivation of azathioprine. Mol Pharmacol 2006;70:747-54.

20. Seidegård J, Vorachek WR, Pero RW, Pearson WR. Hereditary differences in the expression of the human glutathione transferase active on trans-stilbene oxide are due to a gene deletion. Proc Natl Acad Sci U S A 1988;85:7293-7.

21. McLellan RA, Oscarson M, Alexandrie AK, Seidegård J, Evans DA, Rannug A, Ingelman-Sundberg $M$. Characterization of a human glutathione S-transferase mu cluster containing a duplicated GSTM1 gene that causes ultrarapid enzyme activity. Mol Pharmacol 1997;52:958-65. 
22. Stocco G, Martelossi S, Barabino A, Decorti G, Bartoli F, Montico M, Gotti A, Ventura A. Glutathione-Stransferase genotypes and the adverse effects of azathioprine in young patients with inflammatory bowel disease. Inflamm Bowel Dis 2007;13:57-64.

23. Parks DA, Granger DN. Xanthine oxidase: biochemistry, distribution and physiology. Acta Physiol Scand Suppl 1986;548:87-99.

24. Zimm S, Collins JM, O'Neill D, Chabner BA, Poplack DG. Inhibition of first-pass metabolism in cancer chemotherapy: interaction of 6-mercaptopurine and allopurinol. Clin Pharmacol Ther 1983;34:810-7.

25. Pacher $P$, Nivorozhkin A, Szabo $C$. Therapeutic effects of xanthine oxidase inhibitors: renaissance half a century after the discovery of allopurinol. Pharmacol Rev 2006;58:87-114.

26. Pritsos CA. Cellular distribution, metabolism and regulation of the xanthine oxidoreductase enzyme system. Chem Biol Interact 2000;129:195-208.

27. Podolsky DK. Inflammatory bowel disease. N Engl J Med 2002;347:417-29.

28. Wong DR, Derijks LJ, den Dulk MO, Gemmeke EH, Hooymans PM. The role of xanthine oxidase in thiopurine metabolism: a case report. Ther Drug Monit 2007;29:845-8.

29. Guerciolini R, Szumlanski C, Weinshilboum RM. Human liver xanthine oxidase: nature and extent of individual variation. Clin Pharmacol Ther 1991;50:663-72.

30. Relling MV, Lin JS, Ayers GD, Evans WE. Racial and gender differences in N-acetyltransferase, xanthine oxidase, and CYP1A2 activities. Clin Pharmacol Ther 1992;52:643-58.

31. Saruwatari J, Nakagawa K, Shindo J, Tajiri T, Fujieda M, Yamazaki H, Kamataki T, Ishizaki T. A population phenotyping study of three drug-metabolizing enzymes in Kyushu, Japan, with use of the caffeine test. Clin Pharmacol Ther 2002;72:200-8.

32. Aklillu E, Carrillo JA, Makonnen E, Bertilsson L, Ingelman-Sundberg M. Xanthine oxidase activity is influenced by environmental factors in Ethiopians. Eur J Clin Pharmacol 2003;59:533-6.

33. Chocair P, Duley J, Simmonds HA, Cameron JS, Ianhez L, Arap S, Sabbaga E. Low-dose allopurinol plus azathioprine/cyclosporin/ prednisolone, a novel immunosuppressive regimen. Lancet 1993;342:83-4.

34. Kennedy DT, Hayney MS, Lake KD. Azathioprine and allopurinol: the price of an avoidable drug interaction. Ann Pharmacother 1996;30:951-4.

35. Deininger M, Szumlanski CL, Otterness DM, Van Loon J, Ferber W, Weinshilboum RM. Purine substrates for human thiopurine methyltransferase. Biochem Pharmacol 1994;48:2135-8.

36. Kudo M, Moteki T, Sasaki T, Konno Y, Ujiie S, Onose A, Mizugaki M, Ishikawa M, Hiratsuka M. Functional characterization of human xanthine oxidase allelic variants. Pharmacogenet Genomics 2008;18:243-51.

37. Hawwa AF, Millership JS, Collier PS, Vandenbroeck K, McCarthy A, Dempsey S, Cairns C, Collins J, Rodgers C, McElnay JC. Pharmacogenomic studies of the anticancer and immunosuppressive thiopurines mercaptopurine and azathioprine. Br J Clin Pharmacol 2008;66:517-28.

38. Krynetski EY, Evans WE. Genetic polymorphism of thiopurine S-methyltransferase: molecular mechanisms and clinical importance. Pharmacology 2000;61:136-46.

39. Lennard L, Davies HA, Lilleyman JS. Is 6-thioguanine more appropriate than 6-mercaptopurine for children with acute lymphoblastic leukaemia? Br J Cancer 1993;68:186-90.

40. Weinshilboum RM, Sladek SL. Mercaptopurine pharmacogenetics: monogenic inheritance of erythrocyte thiopurine methyltransferase activity. Am J Hum Genet 1980;32:651-62.

41. Schaeffeler E, Lang T, Zanger UM, Eichelbaum M, Schwab M. High-throughput genotyping of thiopurine S-methyltransferase by denaturing HPLC. Clin Chem 2001;47:548-55.

42. Schaeffeler E1, Fischer C, Brockmeier D, Wernet D, Moerike K, Eichelbaum M, Zanger UM, Schwab M. Comprehensive analysis of thiopurine S-methyltransferase phenotype-genotype correlation in a large population of German-Caucasians and identification of novel TPMT variants. Pharmacogenetics 2004;14:407-17.

43. Hamdan-Khalil R, Gala JL, Allorge D, Lo-Guidice JM, Horsmans Y, Houdret N, Broly F. Identification and functional analysis of two rare allelic variants of the thiopurine S-methyltransferase gene, TPMT*16 and TPMT*19. Biochem Pharmacol 2005;69:525-9.

44. Schaeffeler E, Eichelbaum M, Reinisch W, Zanger UM, Schwab M. Three novel thiopurine Smethyltransferase allelic variants $\left(\mathrm{TPMT}^{*} 20, * 21, * 22\right)$ - association with decreased enzyme function. Hum Mutat 2006;27:976.

45. Garat A, Cauffiez C, Renault N, Lo-Guidice JM, Allorge D, Chevalier D, Houdret N, Chavatte P, Loriot MA, Gala JL, Broly F. Characterisation of novel defective thiopurine S-methyltransferase allelic variants. Biochem Pharmacol 2008;76:404-15. 
46. Ujiie S, Sasaki T, Mizugaki M, Ishikawa M, Hiratsuka M. Functional characterization of 23 allelic variants of thiopurine S-methyltransferase gene (TPMT*2 - *24). Pharmacogenet Genomics 2008;18:887-93.

47. Yan L, Zhang S, Eiff B, Szumlanski CL, Powers M, O'Brien JF, Weinshilboum RM. Thiopurine methyltransferase polymorphic tandem repeat: genotype-phenotype correlation analysis. Clin Pharmacol Ther 2000;68:210-9.

48. Alves S, Amorim A, Ferreira F, Prata MJ. Influence of the variable number of tandem repeats located in the promoter region of the thiopurine methyltransferase gene on enzymatic activity. Clin Pharmacol Ther 2001;70:165-74.

49. Tai HL, Krynetski EY, Schuetz EG, Yanishevski Y, Evans WE. Enhanced proteolysis of thiopurine S-methyltransferase (TPMT) encoded by mutant alleles in humans (TPMT*3A, TPMT*2): mechanisms for the genetic polymorphism of TPMT activity. Proc Natl Acad Sci U S A 1997;94:6444-9.

50. Roberts RL, Gearry RB, Bland MV, Sies CW, George PM, Burt M, Marinaki AM, Arenas M, Barclay ML, Kennedy MA. Trinucleotide repeat variants in the promoter of the thiopurine S-methyltransferase gene of patients exhibiting ultra-high enzyme activity. Pharmacogenet Genomics 2008;18:434-8.

51. Weinshilboum R. Thiopurine pharmacogenetics: clinical and molecular studies of thiopurine methyltransferase. Drug Metab Dispos 2001;29:601-5.

52. Reuther LO, Sonne J, Larsen N, Dahlerup JF, Thomsen OO, Schmiegelow K. Thiopurine methyltransferase genotype distribution in patients with Crohn's disease. Aliment Pharmacol Ther 2003;17:65-8.

53. Yates CR, Krynetski EY, Loennechen T, Fessing MY, Tai HL, Pui CH, Relling MV, Evans WE. Molecular diagnosis of thiopurine S-methyltransferase deficiency: genetic basis for azathioprine and mercaptopurine intolerance. Ann Intern Med 1997;126:608-14.

54. Coulthard SA, Hall AG. Recent advances in the pharmacogenomics of thiopurine methyltransferase. Pharmacogenomics J 2001;1:254-61.

55. Ansari A, Hassan C, Duley J, Marinaki A, Shobowale-Bakre EM, Seed P, Meenan J, Yim A, Sanderson J. Thiopurine methyltransferase activity and the use of azathioprine in inflammatory bowel disease. Aliment Pharmacol Ther 2002;16:1743-50.

56. Evans WE. Pharmacogenetics of thiopurine S-methyltransferase and thiopurine therapy. Ther Drug Monit 2004;26:186-91.

57. Kurzawski M, Gawronska-Szklarz B, Drozdzik M. Frequency distribution of thiopurine s-methyltransferase alleles in a polish population. Ther Drug Monit 2004;26:541-5.

58. Kubota T, Chiba K. Frequencies of thiopurine S-methyltransferase mutant alleles (TPMT*2, *3A, *3B and ${ }^{*} 3 \mathrm{C}$ ) in 151 healthy Japanese subjects and the inheritance of TPMT*3C in the family of a propositus. Br J Clin Pharmacol 2001;51:475-7.

59. Ameyaw MM, Collie-Duguid ES, Powrie RH, Ofori-Adjei D, McLeod HL. Thiopurine methyltransferase alleles in British and Ghanaian populations. Hum Mol Genet 1999;8:367-70.

60. Collie-Duguid ES, Pritchard SC, Powrie RH, Sludden J, Collier DA, Li T, McLeod HL.. The frequency and distribution of thiopurine methyltransferase alleles in Caucasian and Asian populations. Pharmacogenetics 1999;9:37-42.

61. Sanderson J, Ansari A, Marinaki T, Duley J. Thiopurine methyltransferase: should it be measured before commencing thiopurine drug therapy? Ann Clin Biochem 2004;41:294-302.

62. Pettersson B, Almer S, Albertioni F, Söderhäll S, Peterson C. Differences between children and adults in thiopurine methyltransferase activity and metabolite formation during thiopurine therapy: possible role of concomitant methotrexate. Ther Drug Monit 2002;24:351-8.

63. Indjova D, Atanasova S, Shipkova M, Armstrong VW, Oellerich M, Svinarov D. Phenotypic and genotypic analysis of thiopurine s-methyltransferase polymorphism in the bulgarian population. Ther Drug Monit 2003;25:631-6.

64. Dilger K, Schaeffeler E, Lukas M, Strauch U, Herfarth H, Müller R, Schwab M.. Monitoring of thiopurine methyltransferase activity in postsurgical patients with Crohn's disease during 1 year of treatment with azathioprine or mesalazine. Ther Drug Monit 2007;29:1-5.

65. Krynetski EY, Schuetz JD, Galpin AJ, Pui CH, Relling MV, Evans WE. A single point mutation leading to loss of catalytic activity in human thiopurine S-methyltransferase. Proc Natl Acad Sci U S A 1995;92: 949-53. 
66. Indjova D, Shipkova M, Atanasova S, Niedmann PD, Armstrong VW, Svinarov D, Oellerich $M$. Determination of thiopurine methyltransferase phenotype in isolated human erythrocytes using a new simple nonradioactive HPLC method. Ther Drug Monit 2003;25:637-44.

67. Schwab M, Schaeffeler E, Marx C, Zanger U, Aulitzky W, Eichelbaum M. Shortcoming in the diagnosis of TPMT deficiency in a patient with Crohn's disease using phenotyping only. Gastroenterology 2001; 121:498-9.

68. Louis E, Belaiche J. Optimizing treatment with thioguanine derivatives in inflammatory bowel disease. Best Pract Res Clin Gastroenterol 2003;17:37-46.

69. Dubinsky MC, Yang H, Hassard PV, Seidman EG, Kam LY, Abreu MT, Targan SR, Vasiliauskas EA. 6-MP metabolite profiles provide a biochemical explanation for 6-MP resistance in patients with inflammatory bowel disease. Gastroenterology 2002;122:904-15.

70. Black AJ, McLeod HL, Capell HA. Thiopurine methyltransferase genotype predicts therapy-limiting severe toxicity from azathioprine. Annals of Internal Medicine 1998;129:716-8.

71. Zelinkova Z, Derijks L, Stokkers PC, Vogels EW, van Kampen AH, Curvers WL, Cohn D, van Deventer SJ, Hommes DW. Inosine triphosphate pyrophosphatase and thiopurine s-methyltransferase genotypes relationship to azathioprine-induced myelosuppression. Clin Gastroenterol Hepatol 2006;4:44-9.

72. Campbell S, Kingstone K, Ghosh S. Relevance of thiopurine methyltransferase activity in inflammatory bowel disease patients maintained on low-dose azathioprine. Aliment Pharmacol Ther 2002;16:389-98.

73. Regueiro M, Mardini H. Determination of thiopurine methyltransferase genotype or phenotype optimizes initial dosing of azathioprine for the treatment of Crohn's disease. J Clin Gastroenterol 2002; 35:240-4.

74. Sandborn WJ. Rational dosing of azathioprine and 6-mercaptopurine. Gut 2001;48:591-2.

75. Seidman EG. Clinical use and practical application of TPMT enzyme and 6-mercaptopurine metabolite monitoring in IBD. Rev Gastroenterol Disord 2003;3 Suppl 1:S30-8.

76. Kaskas BA, Louis E, Hindorf U, Schaeffeler E, Deflandre J, Graepler F, Schmiegelow K, Gregor M, Zanger UM, Eichelbaum M, Schwab M. Safe treatment of thiopurine S-methyltransferase deficient Crohn's disease patients with azathioprine. Gut 2003;52:140-2.

77. Derijks LJ, van Helden RB, Hommes DW, Stokkers PC. Dosing azathioprine in thiopurine S-methyltransferase deficient inflammatory bowel disease patients. Gut 2008;57:872.

78. Colombel JF, Ferrari N, Debuysere H, Marteau P, Gendre JP, Bonaz B, Soulé JC, Modigliani R, Touze Y, Catala P, Libersa C, Broly F. Genotypic analysis of thiopurine S-methyltransferase in patients with Crohn's disease and severe myelosuppression during azathioprine therapy. Gastroenterology 2000; 118:1025-30.

79. Gearry RB, Barclay ML, Burt MJ, Collett JA, Chapman BA, Roberts RL, Kennedy MA. Thiopurine S-methyltransferase (TPMT) genotype does not predict adverse drug reactions to thiopurine drugs in patients with inflammatory bowel disease. Aliment Pharmacol Ther 2003;18:395-400.

80. iest VL, Begg EJ, Gardiner SJ, Frampton CM, Gearry RB, Barclay ML, Clark DW, Hansen P. Pharmacoeconomic analyses of azathioprine, methotrexate and prospective pharmacogenetic testing for the management of inflammatory bowel disease. Pharmacoeconomics 2006;24:767-81.

81. Winter J, Walker A, Shapiro D, Gaffney D, Spooner RJ, Mills PR. Cost-effectiveness of thiopurine methyltransferase genotype screening in patients about to commence azathioprine therapy for treatment of inflammatory bowel disease. Aliment Pharmacol Ther 2004;20:593-9.

82. Kitiyakara T, Hayat J, McIntyre AS. Cost-effectiveness of thiopurine methyltransferase genotype screening in IBD. Aliment Pharmacol Ther 2005;21:790-1; author reply 791-2.

83. Sayani FA, Prosser C, Bailey RJ, Jacobs P, Fedorak RN. Thiopurine methyltransferase enzyme activity determination before treatment of inflammatory bowel disease with azathioprine: effect on cost and adverse events. Can J Gastroenterol 2005;19:147-51.

84. http://www.clinicaltrial.gov.

85. Fraser JH, Meyers H, Henderson JF, Brox LW, McCoy EE. Individual variation in inosine triphosphate accumulation in human erythrocytes. Clin Biochem 1975;8:353-64.

86. Marinaki AM, Ansari A, Duley JA, Arenas M, Sumi S, Lewis CM, Shobowale-Bakre el-M, Escuredo E, Fairbanks LD, Sanderson JD. Adverse drug reactions to azathioprine therapy are associated with polymorphism in the gene encoding inosine triphosphate pyrophosphatase (ITPase). Pharmacogenetics 2004;14:181-7. 
87. Sumi S, Marinaki AM, Arenas M, Fairbanks L, Shobowale-Bakre M, Rees DC, Thein SL, Ansari A, Sanderson J, De Abreu RA, Simmonds HA, Duley JA. Genetic basis of inosine triphosphate pyrophosphohydrolase deficiency. Hum Genet 2002;111:360-7.

88. Marinaki AM, Sumi S, Arenas M, Fairbanks L, Harihara S, Shimizu K, Ueta A, Duley JA. Allele frequency of inosine triphosphate pyrophosphatase gene polymorphisms in a Japanese population. Nucleosides Nucleotides Nucleic Acids 2004;23:1399-401.

89. Duley JA, Simmonds HA, Hopkinson DA, Levinsky RJ. Inosine triphosphate pyrophosphohydrolase deficiency in a kindred with adenosine deaminase deficiency. Clin Chim Acta 1990;188:243-52.

90. Gearry RB, Roberts RL, Barclay ML, Kennedy MA. Lack of association between the ITPA 94C>A polymorphism and adverse effects from azathioprine. Pharmacogenetics 2004;14:779-81.

91. Allorge D, Hamdan R, Broly F, Libersa C, Colombel JF. ITPA genotyping test does not improve detection of Crohn's disease patients at risk of azathioprine/6-mercaptopurine induced myelosuppression. Gut 2005;54:565.

92. UpToDate. In; 2008.

93. Genetics Home Reference website. In; 2008.

94. Wilson JM, Young AB, Kelley WN. Hypoxanthine-guanine phosphoribosyltransferase deficiency. The molecular basis of the clinical syndromes. N Engl J Med 1983;309:900-10.

95. Mak BS, Chi CS, Tsai CR, Lee WJ, Lin HY. New mutations of the HPRT gene in Lesch-Nyhan syndrome. Pediatr Neurol 2000;23:332-5.

96. Puig JG, Torres RJ, Mateos FA, Arcas J, Buño A, Pascual-Castroviejo I. The spectrum of HGPRT deficiency. Clinical experience based on 20 patients from 16 Spanish families. Adv Exp Med Biol 1998;431:25-9.

97. Mateos EA, Puig JG. Purine metabolism in Lesch-Nyhan syndrome versus Kelley-Seegmiller syndrome. J Inherit Metab Dis 1994;17:138-42.

98. Gu JJ, Spychala J, Mitchell BS. Regulation of the human inosine monophosphate dehydrogenase type I gene. Utilization of alternative promoters. J Biol Chem 1997;272:4458-66.

99. Natsumeda Y, Ohno S, Kawasaki H, Konno Y, Weber G, Suzuki K. Two distinct cDNAs for human IMP dehydrogenase. J Biol Chem 1990;265:5292-5.

100. Senda $M$, Natsumeda $Y$. Tissue-differential expression of two distinct genes for human IMP dehydrogenase (E.C.1.1.1.205). Life Sci 1994;54:1917-26.

101. Roberts RL, Gearry RB, Barclay ML, Kennedy MA. IMPDH1 promoter mutations in a patient exhibiting azathioprine resistance. Pharmacogenomics J 2007;7:312-7.

102. Haglund S, Taipalensuu J, Peterson C, Almer S. IMPDH activity in thiopurine-treated patients with inflammatory bowel disease - relation to TPMT activity and metabolite concentrations. $\mathrm{Br} \mathrm{J} C l i n$ Pharmacol 2008;65:69-77.

103. Schinkel $\mathrm{AH}$. The physiological function of drug-transporting P-glycoproteins. Semin Cancer Biol 1997; 8:161-70.

104. Mendoza JL, Urcelay E, Lana R, Martín MC, López N, Guijarro LG, Mayol JA, Taxonera C, de la Concha EG, Peña AS, Díaz-Rubio M. MDR1 polymorphisms and response to azathioprine therapy in patients with Crohn's disease. Inflamm Bowel Dis 2007;13:585-90.

105. Annese V, Valvano MR, Palmieri O, Latiano A, Bossa F, Andriulli A. Multidrug resistance 1 gene in inflammatory bowel disease: a meta-analysis. World J Gastroenterol 2006;12:3636-44.

106. Farrell RJ, Murphy A, Long A, Donnelly S, Cherikuri A, O'Toole D, Mahmud N, Keeling PW, Weir DG, Kelleher D. High multidrug resistance (P-glycoprotein 170) expression in inflammatory bowel disease patients who fail medical therapy. Gastroenterology 2000;118:279-88.

107. Anglicheau D, Thervet E, Etienne I, Hurault De Ligny B, Le Meur Y, Touchard G, Büchler M, Laurent-Puig $P$, Tregouet D, Beaune P, Daly A, Legendre C, Marquet P. CYP3A5 and MDR1 genetic polymorphisms and cyclosporine pharmacokinetics after renal transplantation. Clin Pharmacol Ther 2004;75:422-33.

108. Anglicheau D, Verstuyft C, Laurent-Puig P, Becquemont L, Schlageter $\mathrm{MH}$, Cassinat B, Beaune $\mathrm{P}$, Legendre $C$, Thervet $E$. Association of the multidrug resistance-1 gene single-nucleotide polymorphisms with the tacrolimus dose requirements in renal transplant recipients. J Am Soc Nephrol 2003;14: 1889-96.

109. Norris MD, De Graaf D, Haber M, Kavallaris M, Madafiglio J, Gilbert J, Kwan E, Stewart BW, Mechetner EB, Gudkov AV, Roninson IB. Involvement of MDR1 P-glycoprotein in multifactorial resistance to methotrexate. Int J Cancer 1996;65:613-9. 
110. Zeng H, Lin ZP, Sartorelli AC. Resistance to purine and pyrimidine nucleoside and nucleobase analogs by the human MDR1 transfected murine leukemia cell line L1210/VMDRC.06. Biochem Pharmacol 2004;68:911-21.

111. Wijnholds J, Mol CA, van Deemter L, de Haas M, Scheffer GL, Baas F, Beijnen JH, Scheper RJ, Hatse S, De Clercq $E$, Balzarini J, Borst $P$. Multidrug-resistance protein 5 is a multispecific organic anion transporter able to transport nucleotide analogs. Proc Natl Acad Sci U S A 2000;97:7476-81.

112. Zhou SF, Wang LL, Di YM, Xue CC, Duan W, Li CG, Li Y. Substrates and inhibitors of human multidrug resistance associated proteins and the implications in drug development. Curr Med Chem 2008; 15:1981-2039.

113. Wielinga PR, Reid G, Challa EE, van der Heijden I, van Deemter L, de Haas M, Mol C, Kuil AJ, Groeneveld E, Schuetz JD, Brouwer C, De Abreu RA, Wijnholds J, Beijnen JH, Borst P. Thiopurine metabolism and identification of the thiopurine metabolites transported by MRP4 and MRP5 overexpressed in human embryonic kidney cells. Mol Pharmacol 2002;62:1321-31.

114. de Boer NK, Wong DR, Jharap B, de Graaf P, Hooymans PM, Mulder CJ, Rijmen F, Engels LG, van Bodegraven AA. Dose-dependent influence of 5-aminosalicylates on thiopurine metabolism. Am J Gastroenterol 2007;102:2747-53.

115. Cuffari C, Théorêt Y, Latour S, Seidman G. 6-Mercaptopurine metabolism in Crohn's disease: correlation with efficacy and toxicity. Gut 1996;39:401-6.

116. Derijks LJ, Gilissen LP, Engels LG, Bos LP, Bus PJ, Lohman JJ, Curvers WL, Van Deventer SJ, Hommes DW, Hooymans PM. Pharmacokinetics of 6-mercaptopurine in patients with inflammatory bowel disease: implications for therapy. Ther Drug Monit 2004;26:311-8.

117. KNMP Kennisbank.nl; accessed on 22 Dec 2008. 



\section{Chapter 3}

Screening for TPMT genotype followed by personalised dosing of azathioprine and mercaptopurine in inflammatory bowel disease patients: a randomised trial

MJH Coenen, DJ de Jong, CJ van Marrewijk, LJ Derijks, SH Vermeulen, DR Wong, OH Klungel, ALM Verbeek, PM Hooymans, WHM Peters, RHM te Morsche, WG Newman, H Scheffer, HJ Guchelaar, B Franke

Submitted 


\section{Abstract}

Importance

More than $20 \%$ of inflammatory bowel disease (IBD) patients discontinue thiopurine therapy due to severe adverse drug reactions, among which leukopenia is one of the most serious. Thiopurine S-methyltransferase (TPMT) pharmacogenetic testing seems effective in small studies for optimising thiopurine safety and efficacy. Nonetheless, in clinical practice it is only used on a limited scale.

Objective

To investigate the effectiveness of TPMT genotyping prior to thiopurine treatment to prevent leukopenia occurrence in patients with IBD.

\section{Design}

A parallel randomised controlled trial ("Thiopurine response Optimisation by Pharmacogenetic testing in Inflammatory bowel disease Clinics" (TOPIC) trial). The study was executed from October 2007 until December 2010 and patients were followed for 20 weeks. Patients and caregivers were blinded for group assignment, although caregivers were able to identify carriers of a genetic variant in the intervention group.

\section{Setting}

Thirty Dutch hospitals.

\section{Participants}

852 thiopurine-naïve IBD patients starting on thiopurine treatment were assessed for eligibility. The analysis included 769 patients who were evaluated for the development of leukopenia.

Interventions: Patients were randomly assigned to standard treatment (control) or pre-treatment screening (intervention) for three common variants in TPMT (TPMT*2, *3A and *3C); patients heterozygous for a TPMT variant received $50 \%$ of the standard thiopurine (azathioprine or 6-mercaptopurine) dose; patients homozygous for the tested variants received $0-10 \%$.

\section{Main outcome}

We compared pre-treatment genotyped patients $(n=399)$ with patients receiving standard treatment $(n=370)$ for the primary outcome, occurrence of leukopenia (leukocyte count $<3.0 \times 10^{9} /$ l) in the follow-up period of 5 months after treatment initiation.

\section{Results}

Thirty-nine patients $(9.8 \%)$ in the intervention and 35 patients $(9.5 \%)$ in the control group carried a TPMT variant. No difference in the incidence of leukopenia was observed between both treatment groups $(n=29(7.3 \%)$ versus $n=29(7.8 \%))$. Among TPMT variant carriers, however, leukopenia occurrence was significantly reduced in the intervention group compared to the control group ( $2.6 \%$ versus $22.9 \%$, relative risk $0.11,95 \% \mathrm{Cl}$ : $0.01-0.85$ ).

\section{Conclusions and relevance}

Overall, prior-to-treatment TPMT screening does not reduce the risk of leukopenia in IBD patients treated with thiopurines, but dose adaptation in TPMT variant carriers does. 


\section{Introduction}

Pharmacogenetic testing to optimise treatment is only applied on a limited scale in clinical practice to date, as large-scale randomised controlled trials (RCTs) proving effectiveness of available tests are largely lacking. ${ }^{1-3}$

Thiopurines are effective to induce and maintain long-term remission in up to $70 \%$ of patients with inflammatory bowel diseases (IBD; Crohn's disease (CD) and ulcerative colitis (UC)). ${ }^{4}$ Azathioprine and 6-mercaptopurine are inactive pro-drugs that need to undergo intracellular conversion to pharmacologically active 6-thioguanine nucleotides (6-TGN) before exerting their cytotoxic action on (overactive) immune cells. Thiopurine S-methyltransferase (TPMT) metabolises thiopurines to inactive metabolites leaving less parent drug to be metabolised to active 6-TGN. ${ }^{5,6}$ Approximately $10 \%$ of Caucasians carry a genetic variant in the TPMT gene, resulting in decreased TPMT enzyme activity and consequently higher 6-TGN levels and a higher risk of potentially life-threatening myelosuppression during thiopurine treatment. ${ }^{7}$

More than $20 \%$ of IBD patients discontinue thiopurine treatment due to (serious) adverse drug events. ${ }^{8,9}$ Early identification of patients at risk of adverse drug reactions (ADRs) may result in safer use of thiopurines. Pharmacogenetic testing for TPMT has been advocated for a long time to optimise thiopurine treatment, but clinical use of prospective TPMT testing has been low, and effectiveness data are lacking. ${ }^{10}$ To date, two TPMT-related RCTs have been performed. However, both studies had relatively small sample sizes ( $N=333$ and 29 ) and no definitive conclusions could be drawn. ${ }^{11,12}$ A recent meta-analysis ( $n=4,306$ patients) suggests that IBD patients with decreased TPMT activity are indeed at increased risk of developing leukopenia compared to patients with normal TPMT activity. ${ }^{6}$

In this RCT ("Thiopurine response Optimisation by Pharmacogenetic testing in Inflammatory bowel disease Clinics" (TOPIC)), we investigated whether pre-treatment TPMT genotyping followed by personalised dosing results in a reduced leukopenia incidence.

\section{Materials and methods}

\section{Patients}

Patients were assessed for eligibility by their gastroenterologist. Patients meeting inclusion criteria were randomly assigned to pre-treatment TPMT genotyping (intervention group) or standard treatment (control group). Inclusion criteria were: age $>18$ years and diagnosis of IBD. Exclusion criteria were: previous use of azathioprine 
or 6-mercaptopurine, co-treatment with allopurinol, leukocyte count $<3.0 \times 10^{9} /$, liver test abnormalities (liver enzymes (alanine transaminase (ALT), aspartate transaminase (AST), alkaline phosphatase (AP), and/or gamma glutamate transpeptidase (GGT)) $\geq 2$ times normal upper limit), or reduced renal function (creatinine serum level $\geq 2$ times normal upper limit), known TPMT enzyme activity or genotype, and current pregnancy. The study protocol was approved by the IRB of the Radboud university medical centre (Commissie Mensgebonden Onderzoek (CMO) Regio Arnhem Nijmegen, protocolnumber 13171), approval for inclusion of patients in other institutes was obtained from institutional ethics committee; all patients provided written informed consent. The study is registered at clinicaltrials.gov (NCT00521950).

\section{Study design}

Thirty Dutch hospitals participated in the parallel RCT. Patients were enrolled by the TOPIC recruitment team. Randomisation was based on a computer-generated schedule per participating centre with a block-size of four. Gastroenterologists and patients were blinded to randomisation. Blood samples for TPMT genotyping and enzyme measurements were collected from every patient before treatment initiation, and were numbered upon arrival at the laboratory. Only patients assigned to the intervention group underwent pre-treatment testing for three common TPMT variants (TPMT*2 (238G >C ( $r$ 1800462)), TPMT*3A (460G>A ( $r$ 1800460) and 719A>G (rs1142345)), and TPMT*3C (719A>G (rs1142345)), accounting for approximately 95\% of the variant alleles observed in Caucasians. ${ }^{7,13,14}$ Turn-around time for genotyping results (intervention group) and dosing-advice (all patients) was five working-days. Patients in the control group and those not carrying a TPMT variant were treated according to standard IBD guidelines (azathioprine $2-2.5 \mathrm{mg} / \mathrm{kg} /$ day or 6-mercaptopurine $1-1.5 \mathrm{mg} / \mathrm{kg} / \mathrm{day})$. Patients in the intervention group carrying a genetic variant received $50 \%$ (heterozygotes) or $0-10 \%$ (homozygotes) of the standard thiopurine dose according to the evidence based guidelines of the Dutch Pharmacogenetics Working Group. ${ }^{15}$ For all patients (intervention and control) a letter containing the dose-advice was sent to the gastroenterologist. The majority of the patients ( $n=730$ (94.9\%)) received an advice for the standard dose according to the Dutch guidelines. The gastroenterologists might have been able to identify patients with a TPMT variant in the intervention group based on the dose-advice. Gastroenterologists were allowed to change thiopurine dose or stop treatment when a side-effect occurred. The following guidelines were provided; consider a dose reduction at a count $\leq 4.0 \times 10^{9} / \mathrm{I}$ and a fast decline of leukocyte count, dose reduction of $50 \%$ at a leukocyte count $\leq 3.0 \times 10^{9} / \mathrm{I}$ and treatment stop at a leukocyte count $<1.0 \times 10^{9} / \mathrm{I}$. Treatment re-challenge was at decision of the gastroenterologist.

Table 3.3 provides an overview of the primary and secondary outcomes of the study. Included patients were followed for 20 weeks after thiopurine treatment initiation. Blood for biochemical measurements was collected at least at 1 week before study 
start and at weeks 1, 2, 4, 6, 8 and 20. Erythrocyte sedimentation rate (ESR) and C-reactive protein (CRP) were measured before treatment start and at $20( \pm 6)$ weeks. Leukocytes, thrombocytes, haemoglobin, haematocrit, MCV (mean corpuscular volume), liver enzymes (ALT, AST, AP, GGT), and amylase or lipase were measured at every visit. At week 8 blood samples were collected for 6-TGN and 6-methylmercaptopurine ribonucleotides (6-MMPR) metabolite measurement; metabolites were assessed after follow-up.

Clinical evaluation was performed before study start and at week 20 ( \pm 6 weeks) to determine disease location and activity. During the follow-up period, clinical information (complications, changes in treatment (e.g. changes of azathioprine/ 6-mercaptopurine dose or co-medication)) was collected when patients had contact with the gastroenterologist, timing and intervals of which were at the discretion of the clinician. Patients received questionnaires concerning disease activity (number of (liquid) stools, abdominal pain, fever, use of anti-diarrheal medication, general wellbeing) 1 week before treatment initiation and at week 20. These data, in combination with clinical measures, were used to calculate the disease activity (Harvey-Bradshaw Index ( $\mathrm{HBI}$ ) for $\mathrm{CD}$ and partial-Mayo for $\mathrm{UC}$ ). For the $\mathrm{HBI}$, we used abdominal mass measured before treatment initiation in case this measure was missing at 20 weeks.

\section{Genotyping}

Genetic analysis was performed in a ISO15189-accredited laboratory (Human Genetics department, Radboud university medical centre, Nijmegen, The Netherlands). Genotyping of three common TPMT variants (TPMT*2, *3A, and *3C, UCSC Genome Browser (genome.ucsc.edu) accession number: NM_000367.3) was performed using Taqman SNP genotyping assays according to the manufacturer protocol (Life Technologies, Bleiswijk, Netherlands (rs1800462: assay-ID:C_12091552_30; rs1800460: assay-ID:C_30634116_20; rs1142345: assay-ID:C_19567_20)). Signals were detected with the 7500 Fast Real-Time PCR system (Life Technologies) and subsequently analysed using Allelic Discrimination software version 1.4 (Life Technologies). All patients in the intervention-arm were genotyped in triplicate. Patients assigned to the control group were genotyped in one batch in duplicate after the follow-up period of 20 weeks. Each genotyping experiment contained at least four positive controls for each TPMT variant. Five percent of samples were genotyped in duplicate (within or between plates); all genotypes were concordant. Sequencing of the protein coding part of the TPMT gene was performed in a subset of patients (Table S3.1).

\section{Enzyme and metabolite measurements}

TPMT enzyme activity and thiopurine metabolites were assessed after the follow-up period had ended. Blood for TPMT enzyme activity measurements was collected before treatment initiation and stored at $-80^{\circ} \mathrm{C}$ until further processing. Enzyme activity was 
measured in red blood cells (RBC) using a high-performance liquid chromatography (HPLC) method described previously. ${ }^{16}$

Blood samples for 6-TGN and 6-MMPR measurement were stored at 2-8 ${ }^{\circ} \mathrm{C}$ immediately and sent to the Department of Clinical Pharmacy \& Toxicology (Orbis Medical Centre, Sittard-Geleen, The Netherlands), where samples were processed and stored at $-20^{\circ} \mathrm{C}$ until required. 6-TGN and 6-MMPR levels were determined with a modified HPLC method as published previously. ${ }^{17}$ Lower limits of quantification for 6-TGN and 6-MMPR metabolite levels were $40 \mathrm{pmol} / 8 \times 10^{8} \mathrm{RBC}$, and $300 \mathrm{pmol} / 8 \times 10^{8} \mathrm{RBC}$, respectively. Inter-assay variability for both 6-TGN and 6-MMPR was less than $10 \%$.

\section{Statistical analysis}

The study was designed to have $80 \%$ power with inclusion of 388 patients per treatment arm and a reduction in leukopenia rate of $50 \%$ (leukopenia rate of $11 \%$ in the non-genotyped and $5.5 \%$ in the genotyped group (two-sided p-value threshold 0.05 )). ${ }^{8}$ The dataset was analysed after exclusion of randomised patients who did not receive the allocated intervention and those who were lost to follow-up (Figure 3.1).

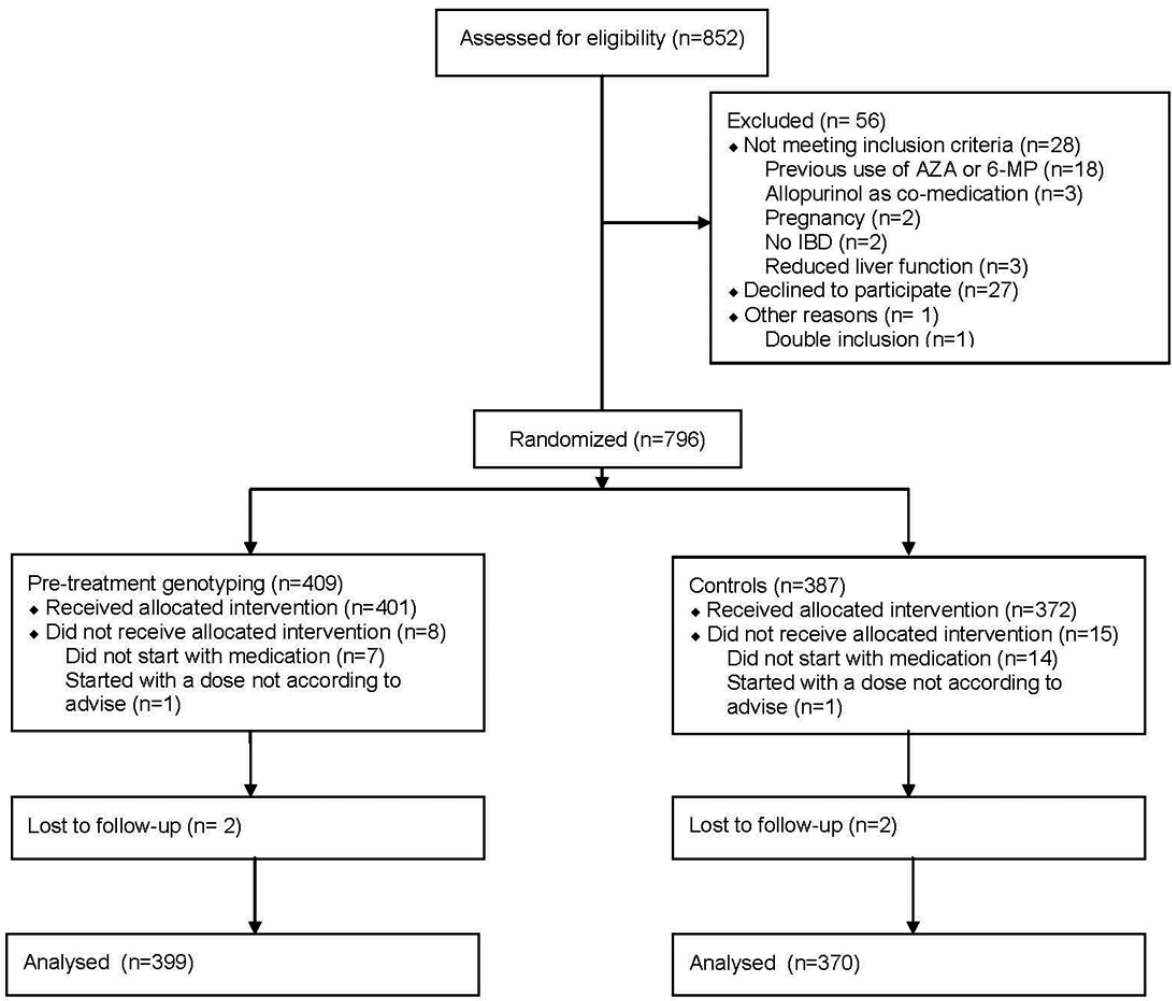

Figure 3.1 Study design of the TOPIC trial. 
Differences in baseline variables and ADRs between patients grouped as intervention or control and with and without TPMT variants were assessed using Pearson chi-square test, Fisher exact tests, Student t-test, Mann-Whitney $U$ test, or independent samples Kruskal-Wallis test, as appropriate. Hardy Weinberg Equilibrium (HWE) was assessed using a chi-square test. The primary outcome of the study was the occurrence of leukopenia defined as a leukocyte count $\leq 3.0 \times 10^{9} /$ / (which is indicative for an increased risk for serious systemic infections) within the follow-up period of $20( \pm 6)$ weeks. Analyses were performed using SPSS package 20.0.0.1.

\section{Results}

Patients were included from October 2007 until December 2010 and followed for a period of 20 weeks, 796 eligible patients were randomised. Final analyses included 399 patients from the intervention and 370 from the control group (Figure 3.1).

Baseline characteristics of intervention and control groups did not show statistically significant differences, except for biologics as co-medication at study start (intervention group 3.5\%, control group 7.6\%, ( $p=0.017)$, Tables 3.1 and Table 3.2).

Table 3.1 Baseline characteristics of the study population that could be analysed for leukopenia.

\begin{tabular}{llll}
\hline & Total & Intervention group & Control group \\
\hline Total & $769(100 \%)$ & $399(100 \%)$ & $370(100 \%)$ \\
Male & $348(45.3 \%)$ & $184(46.1 \%)$ & $164(44.3 \%)$ \\
Age (years (SD)) & $41.2(15.8)$ & $41.7(15.9)$ & $40.5(15.8)$ \\
Weight (kg (SD)) & $74.3(16.1)$ & $73.8(16.0)$ & $74.9(16.2)$ \\
Age of disease onset (years (SD)) & $35.8(15.1)^{1}$ & $36.4(15.4)$ & $35.1(14.8)^{2}$ \\
Disease duration (years (range)) & $1.2(50.4)^{1}$ & $1.3(45.1)$ & $1.2(50.4)^{2}$ \\
Medication & & & \\
Azathioprine & $494(64.2 \%)$ & $252(63.2 \%)$ & $242(65.4 \%)$ \\
6-mercaptopurine & $274(35.6 \%)$ & $146(36.6 \%)$ & $128(34.6 \%)$ \\
None started & $1(0.1 \%)$ & $1(0.3 \%)$ & $0(0 \%)$ \\
Co-medication & & & $301(81.4 \%)$ \\
Corticosteroids & $634(82.4 \%)$ & $333(83.5 \%)$ & $189(51.1 \%)$ \\
$5-a m i n o s a l i c y l a t e s$ & $386(50.2 \%)$ & $197(49.4 \%)$ & $28(7.6 \%)$ \\
Biologicals & $42(5.5 \%)$ & $14(3.5 \%)$ & \\
TPMT genotype & & & $335(90.5 \%)$ \\
$* 1 / * 1$ & $695(90.4 \%)$ & $360(90.2 \%)$ & $3(0.81 \%)$ \\
$* 1 / * 2$ & $7(0.9 \%)$ & $4(1.0 \%)$ & $26(7.0 \%)$ \\
$* 1 / * 3 \mathrm{~A}$ & $56(7.3 \%)$ & $30(7.5 \%)$ & $6(1.6 \%)$ \\
$* 1 / * 3 C$ & $10(1.3 \%)$ & $4(1.0 \%)$ & $0(0 \%)$ \\
$* 3 \mathrm{~A} /{ }^{3} 3 \mathrm{~A}$ & $1(0.1 \%)$ & $1(0.3 \%)$ & \\
\hline
\end{tabular}

Numbers depicted in the table are in mean (standard deviation) or patient number $\mathrm{n}$ (percentage) or median (range) for the disease duration. ${ }^{1} n=768,{ }^{2} n=369 .{ }^{3}$ One patient was homozygous for a variant in the TPMT gene and did not start thiopurine medication in agreement with the therapeutic recommendation. 
Table 3.2 Overview of the patients with CD and UC in the study population that could be analysed for leukopenia.

\begin{tabular}{|c|c|c|c|c|c|c|c|c|c|}
\hline \multirow[t]{2}{*}{ Disease } & \multicolumn{3}{|c|}{ Total population } & \multicolumn{3}{|c|}{ Intervention group } & \multicolumn{3}{|c|}{ Control group } \\
\hline & $\mathrm{CD}$ & UC & IBD-UC & $\mathrm{CD}$ & UC & IBD-UC & $\mathrm{CD}$ & UC & IBD-UC \\
\hline & $\begin{array}{l}467 \\
(60.7 \%)\end{array}$ & $\begin{array}{l}298 \\
(38.8 \%)\end{array}$ & $\begin{array}{l}4 \\
(0.5 \%)\end{array}$ & $\begin{array}{l}240 \\
(60.2 \%)\end{array}$ & $\begin{array}{l}157 \\
(39.3 \%)\end{array}$ & $\begin{array}{l}2 \\
(0.5 \%)\end{array}$ & $\begin{array}{l}227 \\
(61.4 \%)\end{array}$ & $\begin{array}{l}141 \\
(38.1 \%)\end{array}$ & $\begin{array}{l}2 \\
(0.5 \%)\end{array}$ \\
\hline Presence of fistula & $\begin{array}{l}43 \\
(9.3 \%)\end{array}$ & & & $\begin{array}{l}19 \\
(7.9 \%)^{1}\end{array}$ & & & $\begin{array}{l}24 \\
(10.6 \%)^{2}\end{array}$ & & \\
\hline CD known localisation & 457 & & & 234 & & & 223 & & \\
\hline Ileum (L1) & $\begin{array}{l}155 \\
(33.4 \%)\end{array}$ & & & $\begin{array}{l}85 \\
(36.3 \%)\end{array}$ & & & $\begin{array}{l}70 \\
(31.4 \%)\end{array}$ & & \\
\hline Colon (L2) & $\begin{array}{l}111 \\
(23.9 \%)\end{array}$ & & & $\begin{array}{l}52 \\
(22.2 \%)\end{array}$ & & & $\begin{array}{l}59 \\
(26.5 \%)\end{array}$ & & \\
\hline Ileum + colon $(\mathrm{L} 1+\mathrm{L} 2)$ & $\begin{array}{l}191 \\
(41.2 \%)\end{array}$ & & & $\begin{array}{l}97 \\
(41.5 \%)\end{array}$ & & & $\begin{array}{l}94 \\
(42.2 \%)\end{array}$ & & \\
\hline UC known localisation & & 291 & & & 152 & & & 139 & \\
\hline Proctitis ulcerosa (E1) & & $\begin{array}{l}38 \\
(13.1 \%)\end{array}$ & & & $\begin{array}{l}23 \\
(15.1 \%)\end{array}$ & & & $\begin{array}{l}15 \\
(10.8 \%)\end{array}$ & \\
\hline Left sided colitis (E2) & & $\begin{array}{l}121 \\
(41.6 \%)\end{array}$ & & & $\begin{array}{l}67 \\
(44.1 \%)\end{array}$ & & & $\begin{array}{l}54 \\
(38.8 \%)\end{array}$ & \\
\hline Pan-colitis (E3) & & $\begin{array}{l}132 \\
(45.4 \%)\end{array}$ & & & $\begin{array}{l}62 \\
(40.8 \%)\end{array}$ & & & $\begin{array}{l}70 \\
(50.4 \%)\end{array}$ & \\
\hline
\end{tabular}

IBD-UC=IBD unclassified; patient number (n). ${ }^{1} \mathrm{n}=238,{ }^{2} \mathrm{n}=223$.

Steroid use, the main co-medication for patients with IBD, was similar for both groups during follow-up (Table S3.2). Overall, the thiopurine dose was similar for the intervention and control group (Table S3.3). Fifty-five (13.8\%) patients in the intervention and $41(11.1 \%)$ patients in the control group did not start with the advised dose, all but one started with a lower dose (Table S3.4).

One patient, who was homozygous for a TPMT variant, did not start treatment. Thiopurine treatment was discontinued by 167 (41.9\%) patients in the intervention and $143(38.6 \%)$ in the control group; 264 (66.2\%) and $262(70.8 \%)$ of the patients were using thiopurines up to 20 weeks, in the intervention and control group, respectively (Table S3.3).

ADR-frequencies in the first 20 weeks after thiopurine initiation are summarised in Table 3.3. The main outcome measure of our study, leukopenia (leukocyte count $\leq 3.0 \times 10^{9} / \mathrm{I}$ ), was observed in 29 patients in both the intervention and control group. Overall, no significant differences in ADR-frequencies were observed between both groups. Two patients died due to infectious drug-related complications, one in the intervention (heterozygous TPMT ${ }^{*} 3 A$ ) and one in the control group (no variant). 
Table 3.3 Overview of the primary and secondary outcome adverse effects that occurred in the study population.

\begin{tabular}{|c|c|c|c|c|c|c|c|}
\hline & \multicolumn{2}{|c|}{ Total population } & \multicolumn{2}{|c|}{ Intervention group } & \multicolumn{2}{|c|}{ Control group } & \multirow[t]{2}{*}{$\mathrm{RR}(95 \% \mathrm{Cl})$} \\
\hline & $\mathrm{N}(\%)$ & $\mathrm{N}$ total & $\mathrm{N}(\%)$ & $\mathrm{N}$ total & $\mathrm{N}(\%)$ & $\mathrm{N}$ total & \\
\hline \multicolumn{8}{|l|}{ Primary outcome } \\
\hline Leukopenia & $\begin{array}{l}58 \\
(7.5 \%)\end{array}$ & 769 & $\begin{array}{l}29 \\
(7.3 \%)\end{array}$ & 399 & $\begin{array}{l}29 \\
(7.8 \%)\end{array}$ & 370 & $\begin{array}{l}0.93 \\
(0.57-1.52)\end{array}$ \\
\hline \multicolumn{8}{|c|}{ Secondary ADR outcomes based on blood levels } \\
\hline Signs of hepatotoxicity & $\begin{array}{l}203 \\
(26.6 \%)\end{array}$ & 762 & $\begin{array}{l}105 \\
(26.6 \%)\end{array}$ & 394 & $\begin{array}{l}98 \\
(26.6 \%)\end{array}$ & 368 & $\begin{array}{l}1.00 \\
(0.79-1.27)\end{array}$ \\
\hline Signs of pancreatitis & $\begin{array}{l}187 \\
(25.0 \%)\end{array}$ & 749 & $\begin{array}{l}104 \\
(26.9 \%)\end{array}$ & 386 & $\begin{array}{l}83 \\
(22.9 \%)\end{array}$ & 363 & $\begin{array}{l}1.18 \\
(0.92-1.51)\end{array}$ \\
\hline Signs of anaemia & $\begin{array}{l}474 \\
(62.1 \%)\end{array}$ & 763 & $\begin{array}{l}245 \\
(62.0 \%)\end{array}$ & 395 & $\begin{array}{l}229 \\
(62.2 \%)\end{array}$ & 368 & $\begin{array}{l}1.00 \\
(0.89-1.11)\end{array}$ \\
\hline \multicolumn{8}{|c|}{ Secondary ADR outcomes reported by clinicians } \\
\hline General & $\begin{array}{l}318 \\
(41.4 \%)\end{array}$ & 769 & $\begin{array}{l}159 \\
(39.8 \%)\end{array}$ & 399 & $\begin{array}{l}159 \\
(43.0 \%)\end{array}$ & 370 & $\begin{array}{l}0.93 \\
(0.78-1.10)\end{array}$ \\
\hline Gastrointestinal & $\begin{array}{l}549 \\
(71.4 \%)\end{array}$ & 769 & $\begin{array}{l}286 \\
(71.7 \%)\end{array}$ & 399 & $\begin{array}{l}263 \\
(71.1 \%)\end{array}$ & 370 & $\begin{array}{l}1.01 \\
(0.92-1.10)\end{array}$ \\
\hline Infections & $\begin{array}{l}30 \\
(3.9 \%)\end{array}$ & 769 & $\begin{array}{l}13 \\
(3.3 \%)\end{array}$ & 399 & $\begin{array}{l}17 \\
(4.6 \%)\end{array}$ & 370 & $\begin{array}{l}0.71 \\
(0.35-1.44)\end{array}$ \\
\hline Hepatic & $\begin{array}{l}54 \\
(7.0 \%)\end{array}$ & 769 & $\begin{array}{l}27 \\
(6.8 \%)\end{array}$ & 399 & $\begin{array}{l}27 \\
(7.3 \%)\end{array}$ & 370 & $\begin{array}{l}0.93 \\
(0.55-1.55)\end{array}$ \\
\hline Dermatological & $\begin{array}{l}170 \\
(22.1 \%)\end{array}$ & 769 & $\begin{array}{l}83 \\
(20.8 \%)\end{array}$ & 399 & $\begin{array}{l}87 \\
(23.5 \%)\end{array}$ & 370 & $\begin{array}{l}0.88 \\
(0.68-1.15)\end{array}$ \\
\hline Myalgia / Arthralgia & $\begin{array}{l}184 \\
(23.9 \%)\end{array}$ & 769 & $\begin{array}{l}100 \\
(25.1 \%)\end{array}$ & 399 & $\begin{array}{l}84 \\
(22.7 \%)\end{array}$ & 370 & $\begin{array}{l}1.10 \\
(0.86-1.42)\end{array}$ \\
\hline
\end{tabular}

The following reference values were used for the side-effects based on blood levels. Leukopenia: leukocyte count $\leq 3.0 \times 10^{9} /$. Hepatotoxicity: at least one liver enzyme (alkaline phosphatase, alanine aminotransaminase, aspartate aminotransaminase and/or gamma glutamate transpeptidase) $>2 \mathrm{x}$ upper limit of the reference value. Pancreatitis: amylase and/or lipase blood level higher than reference value. Anaemia: haemoglobin lower than reference value.

Clinicians completed case report forms during every hospital visit of the patients for the occurrence of ADRs. In case a specific ADR was reported more than one time in a patient, we included this patient only once. The following ADRs were collected for general side effects: dizziness, shivers, fever, general malaise. Gastrointestinal side effects included: stomach ache, diarrhea, reduced appetite, nausea and vomiting. The group hepatic side effects is composed of cholestasis, cholangitis, hepatitis, and steatosis. Dermatological side-effects are hair loss, warts, and skin rash. In the overall groups presented in the table patients might be counted more than once if they had more than one ADR. RR: relative risk; $95 \% \mathrm{Cl}: 95 \%$ confidence interval.

Observed allele frequencies were comparable with reported frequencies (Table 3.1). ${ }^{14}$ One person $(0.1 \%)$ was homozygous for $T P M T^{*} 3 A$ and 73 patients $(9.5 \%)$ were heterozygous carriers of a TPMT variant. Enzyme activity measurements showed that patients carrying a genetic variant had a lower TPMT enzyme activity than those without a variant (Figure S3.1).

Eleven patients without one of the pre-tested variants had a low TPMT enzyme activity (<60 mg 6-methylguanine/mmolHb.h), one of them developed leukopenia. Complete sequencing of the TPMT gene coding region revealed a known silent variant (rs2842934; lle158lle, TPMT*1S) in four of the eleven patients. ${ }^{18}$ 
No difference in the occurrence in leukopenia was observed between the intervention and control group ( $7.3 \%$ vs. $7.8 \%$, relative risk (RR) $0.93,95 \% \mathrm{Cl}: 0.57-1.52$ ). The median leukocyte counts of the patients with leukopenia were similar between the intervention (median $2.7 \times 10^{9} /$ (range $0.2-3 \times 10^{9} / \mathrm{l}$ )) and control group (median $2.5 \times 10^{9} / \mathrm{I}$ (range $\left.0.9-3 \times 10^{9} / \mathrm{I}\right)$ ). Also, no overall difference in the occurrence of leukopenia was observed based on the intention-to-treat analysis (7.2\% and $6.4 \%$ in the studied groups) and the analysis excluding patients on biologicals (7.3\% versus $6.4 \%$ ).

Analysis of the subgroup of patients carrying a TPMT variant $(* 2, * 3 \mathrm{~A}$ or $* 3 \mathrm{C})$ showed that a personalised dose regimen based on pre-treatment genotyping resulted in a statistically significant decrease in leukopenia occurrence $(p=0.011 ; R R 0.11,95 \% \mathrm{Cl}$ : 0.01-0.85, Table 3.4).

In the intervention group only one out of 39 patients carrying a TPMT variant $(2.6 \%)$ developed leukopenia, compared to 8 of 35 patients in the control group $(22.9 \%)$. Analysis in the subset of patients that had not received biologicals $(n=69)$ also showed fewer instances of leukopenia $(p=0.011)$. No difference in the occurrence of leukopenia between intervention and control groups was found for the patients without a TPMT variant $(\mathrm{p}=0.46$, Table 3.4).

Table 3.4 Secondary analysis: Leukopenia occurrence in the intervention and control group.

\begin{tabular}{llclcl}
\hline & \multicolumn{2}{l}{ Intervention group $(\mathrm{n}=399)$} & \multicolumn{2}{l}{ Control group $(\mathrm{n}=370)$} & RR $(95 \% \mathrm{Cl})$ \\
TPMT variant & Yes $(\mathrm{n}=39)$ & No $(\mathrm{n}=360)$ & Yes $(\mathrm{n}=35)$ & No $(\mathrm{n}=335)$ & \\
\hline Leukopenia & $1(2.6 \%)^{*}$ & & $8(22.9 \%)$ & $21(6.3 \%)$ & $\begin{array}{l}0.11(0.01-0.85) \\
1.2(0.72-2.12)\end{array}$ \\
\hline
\end{tabular}

Leukopenia is defined as a leukocyte count $\leq 3.0 \times 10^{9} / \mathrm{I}$. The three TPMT variants analysed did not show deviations from HWE $(238 \mathrm{G}>\mathrm{C}, \mathrm{p}=0.9,460 \mathrm{G}>\mathrm{A}, \mathrm{p}=0.93719 \mathrm{~A}>\mathrm{G}, \mathrm{p}=0.67)$. The patient homozygous for $T P M T^{*} 3 A$ was randomised to the intervention group, the other patients in the intervention group were heterozygous for TPMT*2 $(n=4), T^{*} M T^{*} 3 A(n=30)$ or TPMT*3C $(n=4)$. The following genotypes were observed in the control group, all patients were heterozygous, TPMT*2 $(n=3), T_{P M T}^{*} 3 A(n=26)$ and TPMT*3C $(n=6)$. *Patient died from leukopenia due to an E. Coli sepsis with pneumonia 3 days after start of thiopurine treatment.

To investigate whether thiopurine dose reduction in TPMT variant carriers in the intervention arm resulted in effective treatment, two approaches were used. First, steady-state 6-TGN and 6-MMPR metabolite levels were assessed at week 8 (Figure 3.2 and Figure S3.2). Comparison of patients with a TPMT variant showed that a reduced thiopurine dose resulted in 6-TGN levels within the therapeutic range, whereas a standard dose resulted in clearly elevated 6-TGN levels (Figure 3.2A). In addition, 6-TGN and 6-MMPR concentrations in patients without a genetic variant did not differ between the groups indicating that both groups were equally adherent to treatment. Highest 6-MMPR concentrations were, as expected, observed in patients without a genetic TPMT variant (Figure 3.2A), followed by patients with a variant on standard thiopurine dose; lowest levels were observed in patients treated with a reduced thiopurine dose. Six patients (one in the intervention and five in the control group) had 
undetectable metabolite levels at week 8 after thiopurine initiation, suggesting noncompliance.
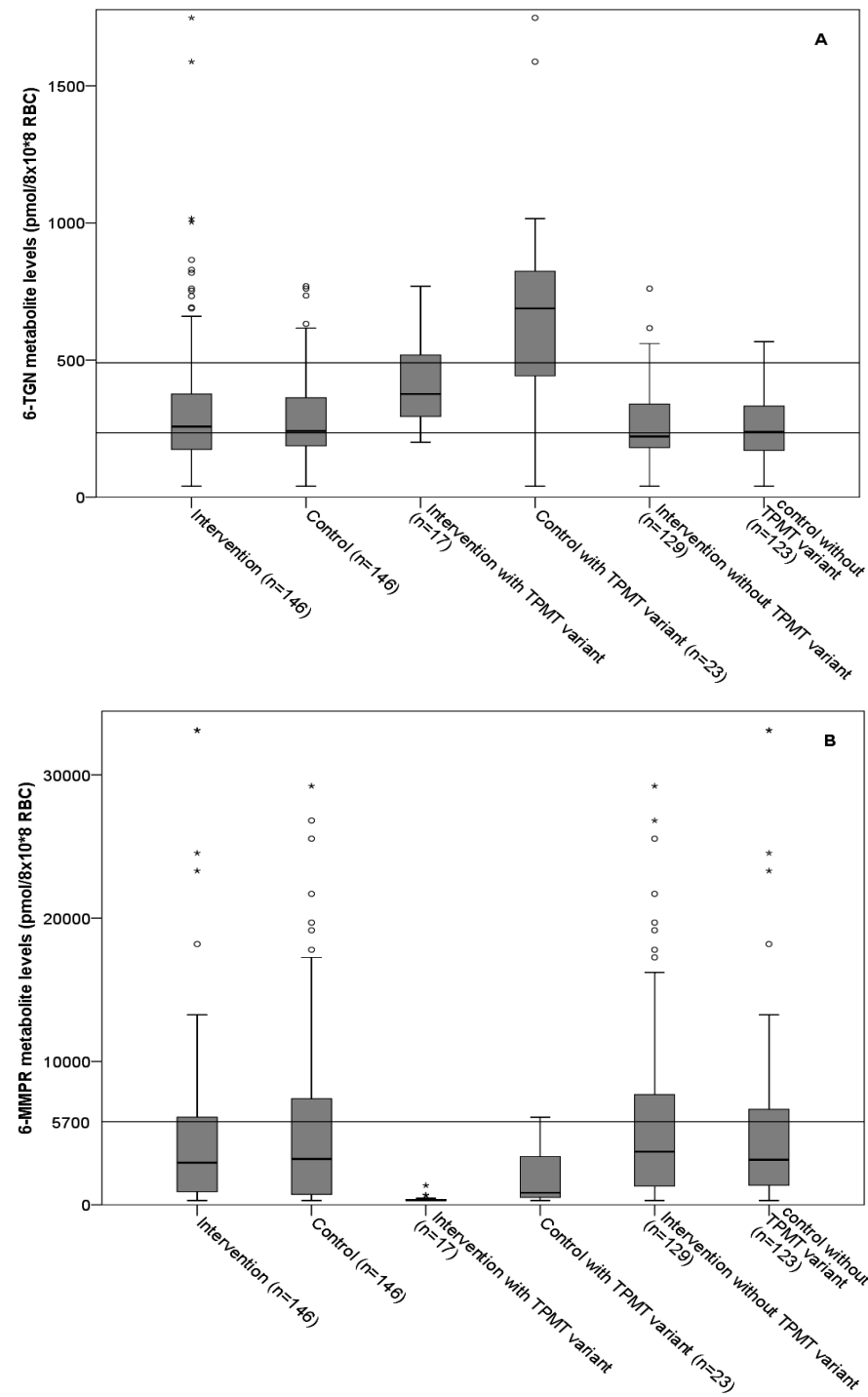

Figure 3.2 Box-plots for steady-state metabolite levels 6-TGN (A) and 6-MMPR (B) in pmol/8x10 RBC.

Metabolite levels are measured at 8 weeks of treatment. The therapeutic range of 6-TGN metabolites (235 and $\left.490 \mathrm{pmol} / 8 \times 10^{8} \mathrm{RBC}\right)$ and normal range levels of 6 -MMPR $(<5700$ $\mathrm{pmol} / 8 \times 10^{8} \mathrm{RBC}$ ) are indicated with horizontal lines in panels $(\mathbf{A})$ and $(\mathbf{B})$, respectively. The boxes indicate the $25^{\text {th }}$ to $75^{\text {th }}$ percentile and the median indicated by a horizontal line in the box. Whiskers indicate 1.5 interquartile ranges. O indicate outliers (more than 1.5 IQR) and * extreme outliers (more than three IQR). The numbers on the $x$-axis indicate the number of patients analysed. A statistically significantly difference in 6-TGN $(p=0.004)$ and 6-MMPR $(p<0.001)$ was observed between the intervention and control group for the patients that carried a genetic variant in TPMT. Similar metabolite levels were observed when excluding patients who had a dose change before 8 weeks of thiopurine therapy (Figure S3.2). 
Secondly, disease activity was assessed. Overall, we did not observe statistically significant differences in disease activity between both groups at baseline $(p=0.12$ for $\mathrm{HBI}$ and $p=0.83$ for partial Mayo) and 20 weeks after treatment initiation ( $p=0.18$ for $\mathrm{HBI}$ and $\mathrm{p}=0.14$ for partial Mayo). A decrease in the median disease activity scores after 20 weeks was observed in both treatment arms, in patients with and without the genetic variant (Figures 3.3A and Figure 3.3B).
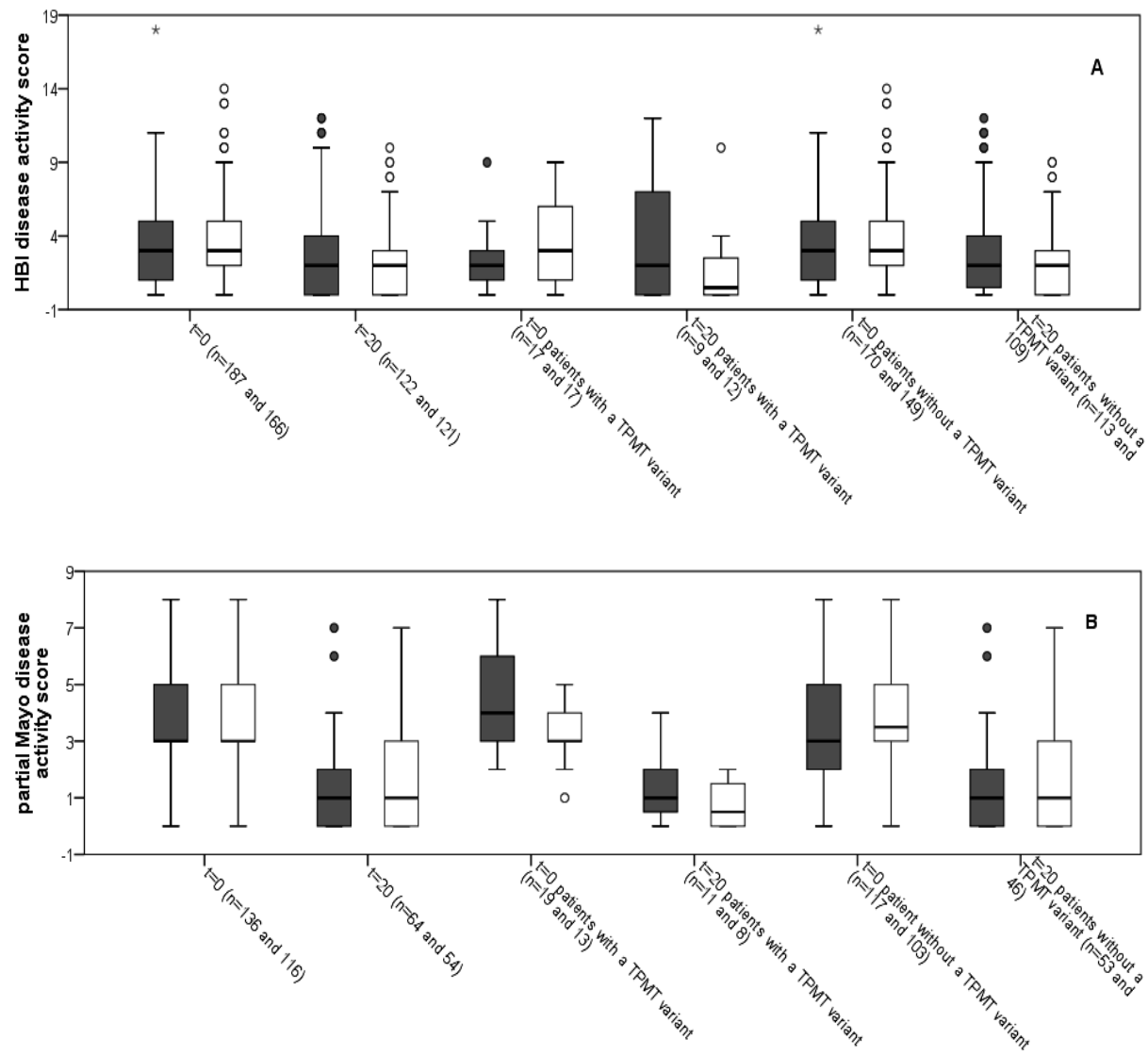

Figure 3.3 Box-plots for disease activity scores for patients with Crohn's disease (HBI (A)) and ulcerative colitis (partial Mayo (B)).

Disease activity scores are given for the intervention (grey bars) and control group (white bars). The HBI can range from 0-18 and the partial Mayo score from 0-9, a higher score means higher disease activity. The numbers given for each set of boxes indicate the number of patients for the intervention and control group, respectively. The boxes indicate the $25^{\text {th }}$ to $75^{\text {th }}$ percentile and the median indicated by a horizontal line in the box. Whiskers indicate 1.5 interquartile range. $O$ indicate outliers (more than $1.5 \mathrm{IQR}$ ) and ${ }^{*}$ extreme outliers (more than three IQR). The numbers on the $x$-axis indicate the number of patients analysed. 


\section{Discussion}

The TOPIC trial, the largest RCT studying the impact of TPMT genotyping prior to thiopurine treatment in IBD patients showed no significant difference in leukopenia risk between the intervention and control group. In addition, no significant differences were observed for the secondary outcome measures. However, TPMT screening significantly reduced the risk of leukopenia in the subgroup of patients with a genetic variant.

Forty percent of the patients discontinued thiopurine treatment due to adverse effects, which is relatively high compared to previous reports. ${ }^{9}$ Taking the patients with a successful re-challenge into account, we observed drop-out rates consistent with those reported in the literature. Despite the high discontinuation rate, we did not observe increased leukopenia frequencies in our population. ${ }^{4}$ Thus, the TOPIC trial accurately reflects the general IBD population treated with thiopurines.

We could not demonstrate a difference in leukopenia occurrence between cases and controls, neither in those receiving treatment nor in the intention-to-treat analysis. However, subgroup analysis in patients with a variant in the TPMT gene showed that the intervention strongly reduced leukopenia frequency from $22.9 \%$ to $2.6 \%$. These findings illustrate the problem in trying to use whole-population randomised studies to investigate the effectiveness of pharmacogenetic testing: the high risk genotype of TPMT constitutes a small portion of the population (here $10 \%$ ), which makes it extremely hard to demonstrate a benefit for all patients; only a portion of the population benefits. Post-hoc power analysis indeed showed that the subgroup analysis was sufficiently powered ( $80 \%$ power with 38 patients), but that an RCT with 42,556 participants would be needed to show a benefit for the entire intervention group (power of $80 \%$ (based on leukopenia incidence observed in our study population)).

Our data confirm the meta-analysis including 47 mainly retrospective studies, which showed an association between decreased TPMT enzyme activity (based on genotype or phenotype) and increased risk for leukopenia. ${ }^{6}$ The meta-analysis also showed that thiopurine treatment should be avoided in patients homozygous for a TPMT variant. ${ }^{11}$ We could not demonstrate this as the one patient homozygous for a genetic variant in TPMT in the intervention group did not start treatment. In agreement with previous reports the TOPIC trial showed that other factors than TPMT genotype also play a role in the development of leukopenia. ${ }^{19}$ It is possible that patients with leukopenia carry genetic variants in TPMT other than those analysed. ${ }^{14}$ However, sequencing of the entire TPMT gene for 11 patients with low TPMT enzyme activity, but negative for the three common TPMT variants, did not reveal other mutations leading to a diminished TPMT enzyme activity. To further improve the prediction of thiopurine-induced leukopenia, it might thus be worthwhile to study additional genes in the thiopurine 
metabolism and include environmental factors. Studies show that analysing variants in two or more genes involved in thiopurine metabolism, including TPMT, may enhance the prediction of leukopenia ${ }^{13,20-24}$, but further large-scale studies are warranted. Beside genetic factors, viral infections and co-medication during thiopurine treatment can induce the development of leukopenia. ${ }^{19,25,26,27}$ These non-genetic factors should be taken into consideration before thiopurine initiation as they might interfere with genotype-guided dosing.

A limitation of our study is that $12.5 \%$ of the patients were not treated according to the advised dose. However, this probably reflects the situation of genotype-guided dosing in the clinical setting.

A possible draw-back of treatment with a reduced thiopurine dose might be the risk for under-treatment, but this was not the case in our study, as treatment efficacy was similar in the two study arms. Patients receiving genotype-guided thiopurine dosereduction had a median steady-state 6 -TGN level within the therapeutic range. ${ }^{28-30}$

Current guidelines for thiopurine treatment mandate regular haematological monitoring to detect (severe) leukopenia. However, this is not a complete safeguard as leukopenia can develop suddenly. ${ }^{31}$ A large meta-analysis in patients with IBD showed that the cumulative incidence of infections is $6.5 \%$ in patients with thiopurine-induced myelotoxicity. ${ }^{4}$ The same study reported a cumulative incidence of mortality of $0.06 \%$ due to azathioprine induced bone marrow suppression and the risk of death among IBD patients with myelotoxicity was $1 \%$. This underscores the importance of treating patients as safe as possible (i.e. based on genotype) from treatment start. A recent cost-effectiveness analysis $(n=333)$ as part of an RCT, showed that pre-treatment TPMT genotyping has a probability of $71 \%$ to be cost-effective. ${ }^{32}$ Importantly, however, the burden of IBD on society is high with peak onset age of 20-30 years for Crohn's disease and 30-40 years for ulcerative colitis, the productivity and patient's quality of life is reduced in many aspects. ${ }^{33}$ Therefore avoidance of leukopenia, even in a few cases should be valued highly.

The results of our TOPIC trial, in combination with literature show that pre-treatment TPMT screening followed by personalised dosing reduces the risk of leukopenia and indicate that pharmacogenetic TPMT testing should be used as standard care to individualise thiopurine treatment of IBD patients. 


\section{Supplementary tables and figures}

Table S3.1 Overview of the primers used for sequencing of the protein-coding part of the TPMT gene.

\begin{tabular}{lll}
\hline Exon & Forward primer & Reverse primer \\
\hline 3 & AGGTTTTCATTTAGTTCATCAAT & TTTTTGATAGAACATTTCTCTATTGT \\
4 & TGAATGAAAAGTGTTCACCTACC & TTTCAAAACTCAATCCAGAAAGA \\
5 & TCTTTGAAACCCTATGAACCTGA & AAAACTTTTGTGGGGATATGGA \\
6 & GCCCTCTTTCCTTGACTATT & GAGGAAGACACCTCCACTCC \\
7 & TGTTGAAGTACCAGCATGCAC & TTCCAAACATAATAACCTATTTCAAAC \\
8 & CGAAAGTAACTTCTGGCTTC & GGCAACTGGTAAAAGAAAAA \\
9 & TGAGAAGAACATGCCACATCA & GCCAGGCCCAAAAGAGTTA \\
10 & CACCCAGCCAATTTTGAGTA & ACAGGTAACACATGCTGATTGG \\
\hline
\end{tabular}

PCR was performed on 10 ng DNA using AmpliTaq Gold 360 mastermix (Life Techologies, Bleiswijk). Annealing temperature was $56^{\circ} \mathrm{C}$ for all exons. Sequencing was performed using Sanger Technology.
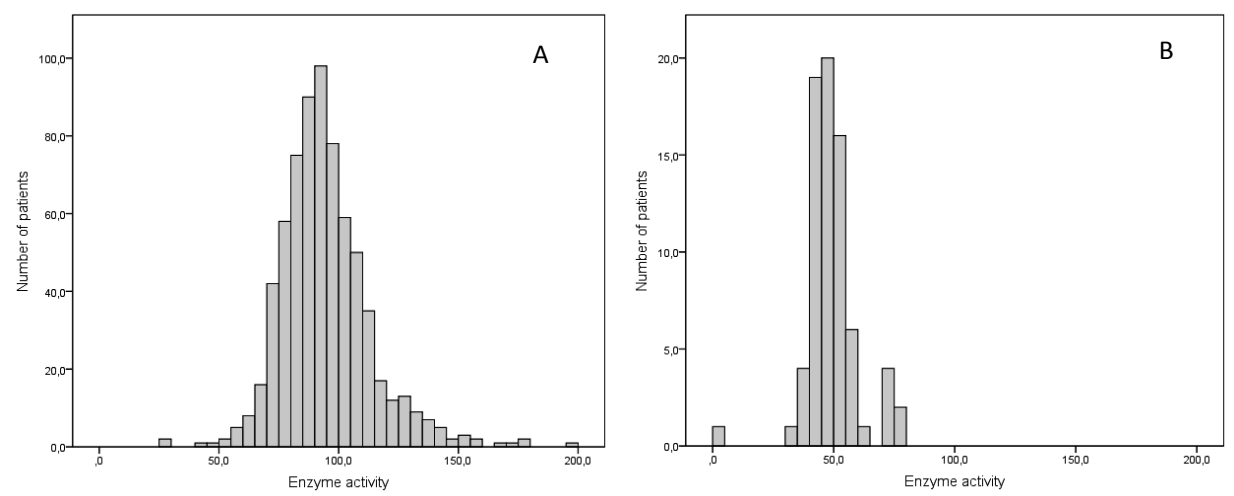

Figure S3.1 TPMT enzyme activity distribution in the study population.

Overview of the enzyme activity $(\mathrm{mg} / \mathrm{mmolHb} . \mathrm{h})$ distribution in patients without a genetic variant (A) and with a genetic variant (B) in the TPMT gene. Each bar represents 5 units of the scale (e.g. 0-5, >5-10). Patients not carrying a TPMT variant had a mean enzyme activity for 6-methylguanine of $94.5 \pm 19.0 \mathrm{mg} / \mathrm{mmolHb} . \mathrm{h}$, and in heterozygous patients the mean TPMT enzyme activity of 6 -methylguanine was $49.8 \pm 8.9 \mathrm{mg} / \mathrm{mmolHb}$.h. The patient homozygous for $T_{P M T}^{*} 3 A$ had an enzyme activity of $0.50 \mathrm{mg} 6$-methylguanine/mmol Hb.h before thiopurine therapy. 
Table S3.2 Corticosteroid use in intervention and control group.

\begin{tabular}{lll}
\hline & $\begin{array}{l}\text { Intervention group } \\
(\mathrm{n}=399)\end{array}$ & $\begin{array}{c}\text { Control group } \\
(\mathrm{n}=370)\end{array}$ \\
\hline $\begin{array}{l}\text { Steroid use at } \mathrm{t}=0 \text { ( } \mathrm{n}(\%)) \\
\text { All steroids (systemic and local) }\end{array}$ & $301(81.4 \%)$ \\
Systemic steroids & $333(83.5 \%)$ & $289(78.1 \%)$ \\
Steroids initiated (n (\%)) & $319(79.9 \%)$ & $64(17.3 \%)$ \\
All steroids (systemic and local) & $72(18.0 \%)$ & $55(14.9 \%)$ \\
Systemic steroids & $54(13.5 \%)$ & $169(45.7 \%)$ \\
Steroids discontinued (n (\%)) & & $155(41.9 \%)$ \\
All steroids (systemic and local) & $170(42.6 \%)$ & $317(85.7 \%)$ \\
Systemic steroids & $159(39.8 \%)$ & $301(81.4 \%)$ \\
Steroids used during follow-up period (n (\%)) & & \\
All steroids (systemic and local) & $346(86.7 \%)$ & $0.71(0.38)$ \\
Systemic steroids & $336(84.2 \%)$ & $0.66(0.40)$ \\
Duration steroid use (mean (SD)) & & \\
All steroids (systemic and local) & $0.71(0.39)$ & \\
Systemic steroids & $0.66(0.40)$ & \\
\hline
\end{tabular}

*Duration calculated as percentage of the study period. Comparisons in steroid use between intervention and control group, using a chi-square test or Mann Whitney-U test for the duration of steroid use, showed no statistically significant differences. 


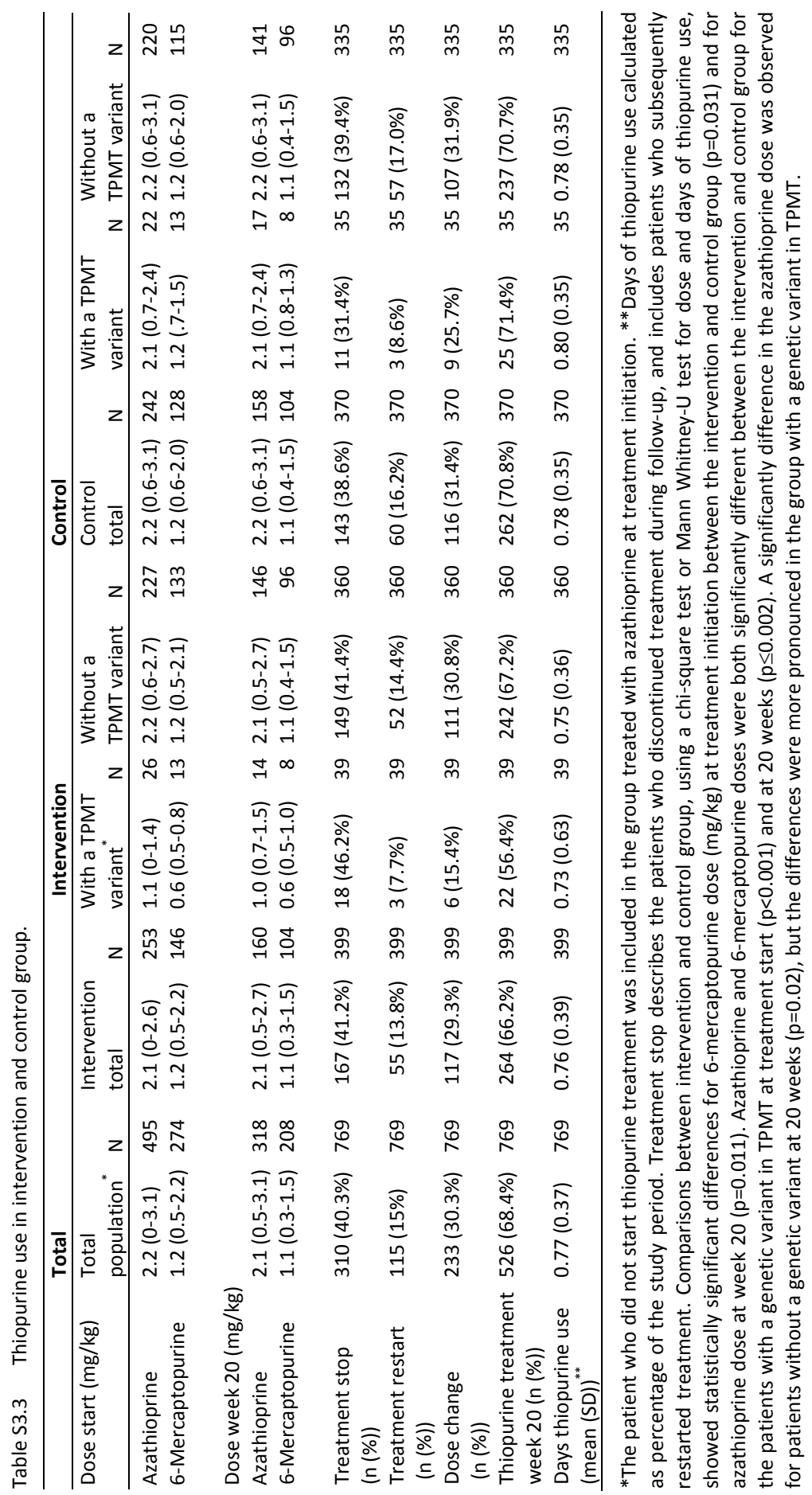


Table S3.4 Patient enrollments per centre and number of patients starting with a dose not according to the provided advice $(\mathrm{n}(\%))$.

\begin{tabular}{|c|c|c|c|}
\hline Centre number & $\begin{array}{l}\text { Total number of } \\
\text { patients included }\end{array}$ & $\begin{array}{l}\text { Patients carrying a } \\
\text { variant in TPMT }^{*}\end{array}$ & $\begin{array}{l}\text { Protocol violations } \\
\text { starting dose }\end{array}$ \\
\hline 1 & 45 (5.9\%) & $4(8.8 \%)$ & $3(6.6 \%)$ \\
\hline 2 & $10(1.3 \%)$ & $0(0 \%)$ & $1(10 \%)$ \\
\hline 3 & 34 (4.4\%) & $4(11.8 \%)$ & $4(11.8 \%)$ \\
\hline 4 & $14(1.8 \%)$ & $1(7.1 \%)$ & $1(7.1 \%)$ \\
\hline 5 & 32 (4.2\%) & $6(18.8 \%)$ & 7 (21.9\%) \\
\hline 6 & $20(2.6 \%)$ & $1(1.4 \%)$ & $1(1.4 \%)$ \\
\hline 7 & 38 (4.9\%) & $4(10.5 \%)$ & $8(4.75 \%)$ \\
\hline 8 & $19(2.5 \%)$ & $2(2.7 \%)$ & $3(15.8 \%)$ \\
\hline 9 & $51(6.6 \%)$ & $5(9.8 \%)$ & $14(27.5 \%)$ \\
\hline 10 & $5(0.7 \%)$ & $1(20 \%)$ & $1(20 \%)$ \\
\hline 11 & $28(3.6 \%)$ & $0(0 \%)$ & $0(0 \%)$ \\
\hline 12 & $58(7.5 \%)$ & 7 (12.1\%) & 7 (12.1\%) \\
\hline 13 & 82 (10.7\%) & $8(9.8 \%)$ & 5 (6.1\%) \\
\hline 14 & 30 (3.9\%) & $4(13.3 \%)$ & $3(10 \%)$ \\
\hline 15 & $51(6.6 \%)$ & $8(15.7 \%)$ & $18(35.3 \%)$ \\
\hline 16 & $10(1.3 \%)$ & $1(10 \%)$ & $0(0 \%)$ \\
\hline 17 & $26(3.4 \%)$ & $2(7.7 \%)$ & $1(3.8 \%)$ \\
\hline 18 & $29(3.8 \%)$ & $3(10.3 \%)$ & $3(10.3 \%)$ \\
\hline 19 & 47 (6.1\%) & $2(4.3 \%)$ & $1(2.1 \%)$ \\
\hline 20 & $2(0.3 \%)$ & $0(0 \%)$ & $0(0 \%)$ \\
\hline 21 & $40(5.2 \%)$ & $2(5.0 \%)$ & $2(5.0 \%)$ \\
\hline 22 & $19(2.5 \%)$ & $2(10.5 \%)$ & $1(5.3 \%)$ \\
\hline 23 & 45 (5.9\%) & $3(6.7 \%)$ & $3(6.7 \%)$ \\
\hline 24 & $1(0.1 \%)$ & $0(0 \%)$ & $0(0 \%)$ \\
\hline 25 & $4(0.5 \%)$ & $0(0 \%)$ & $2(50 \%)$ \\
\hline 26 & $1(0.1 \%)$ & $1(100 \%)$ & $0(0 \%)$ \\
\hline 27 & $18(2.3 \%)$ & 2 (11.1\%) & $6(33.3 \%)$ \\
\hline 28 & $4(0.5 \%)$ & $0(0 \%)$ & $1(25 \%)$ \\
\hline 29 & $3(0.4 \%)$ & $0(0 \%)$ & $0(0 \%)$ \\
\hline 30 & $3(0.4 \%)$ & $1(33.3 \%)$ & $0(0 \%)$ \\
\hline Total & 769 (100\%) & 74 (9.6\%) & 96 (12.5\%) \\
\hline
\end{tabular}

* Percentage of the number of patients included at a particular centre. 

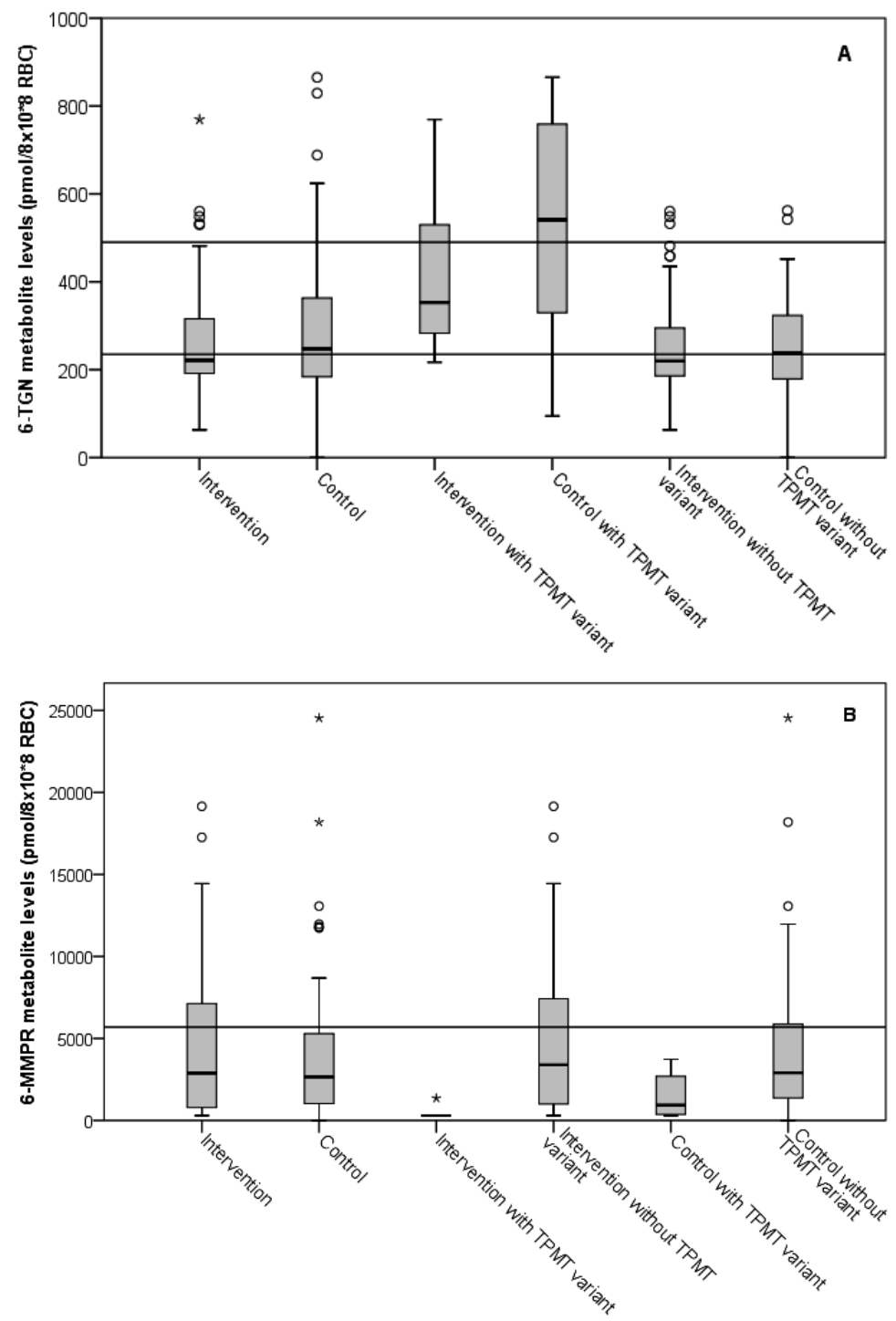

Figure S3.2 Box-plots for steady-state metabolite levels 6-TGN (A) and 6-MMPR (B) after exclusion of patients who received a dose change before 8 weeks of treatment start.

Metabolite levels (in pmol/ $8 \times 10^{8} \mathrm{RBC}$ ) are measured at 8 weeks of treatment. The therapeutic range of 6-TGN metabolites ( 235 and $490 \mathrm{pmol} / 8 \times 10^{8} \mathrm{RBC}$ ) and normal range levels of 6-MMPR $\left(<5,700 \mathrm{pmol} / 8 \times 10^{8} \mathrm{RBC}\right)$ are indicated with horizontal lines in panels $(\mathbf{A})$ and $(\mathbf{B})$, respectively. The boxes indicate the $25^{\text {th }}$ to $75^{\text {th }}$ percentile and the median indicated by a horizontal line in the box. Whiskers indicate 1.5 interquartile ranges. O indicate outliers (more than $1.5 \mathrm{IQR}$ ) and * extreme outliers (more than three IQR). 


\section{References}

1. Pirmohamed M, Burnside G, Eriksson N, Jorgensen AL, Toh CH, Nicholson T, Kesteven P, Christersson C, Wahlström B, Stafberg C, Zhang JE, Leathart JB, Kohnke H, Maitland-van der Zee AH, Williamson PR, Daly AK, Avery P, Kamali F, Wadelius M; EU-PACT Group. A randomized trial of genotype-guided dosing of warfarin. N Engl J Med 2013;369:2294-303.

2. Verhoef TI, Ragia G, de Boer A, Barallon R, Kolovou G, Kolovou V, Konstantinides S, Le Cessie S, Maltezos E, van der Meer FJ, Redekop WK, Remkes M, Rosendaal FR, van Schie RM, Tavridou A, Tziakas D, Wadelius M, Manolopoulos VG, Maitland-van der Zee AH; EU-PACT Group. A randomized trial of genotype-guided dosing of acenocoumarol and phenprocoumon. N Engl J Med 2013;369:2304-12.

3. Mallal S, Phillips E, Carosi G, Molina JM, Workman C, Tomazic J, Jägel-Guedes E, Rugina S, Kozyrev O, Cid JF, Hay P, Nolan D, Hughes S, Hughes A, Ryan S, Fitch N, Thorborn D, Benbow A; PREDICT-1 Study Team. HLA-B*5701 screening for hypersensitivity to abacavir. N Engl J Med 2008;358:568-79.

4. Gisbert JP, Gomollon F. Thiopurine-induced myelotoxicity in patients with inflammatory bowel disease: a review. Am J Gastroenterol 2008;103:1783-800.

5. Ujiie S, Sasaki T, Mizugaki M, Ishikawa M, Hiratsuka M. Functional characterization of 23 allelic variants of thiopurine S-methyltransferase gene (TPMT*2 - *24). Pharmacogenet Genomics 2008;18:887-93.

6. Higgs JE, Payne K, Roberts C, Newman WG. Are patients with intermediate TPMT activity at increased risk of myelosuppression when taking thiopurine medications? Pharmacogenomics 2010;11:177-88.

7. Hindorf $U$, Appell ML. Genotyping should be considered the primary choice for pre-treatment evaluation of thiopurine methyltransferase function. J Crohns Colitis 2012;6:655-9.

8. Ansari A, Hassan C, Duley J, Marinaki A, Shobowale-Bakre EM, Seed P, Meenan J, Yim A, Sanderson J. Thiopurine methyltransferase activity and the use of azathioprine in inflammatory bowel disease. Aliment Pharmacol Ther 2002;16:1743-50.

9. Jharap B, Seinen ML, de Boer NK, van Ginkel JR, Linskens RK, Kneppelhout JC, Mulder CJ, van Bodegraven AA. Thiopurine therapy in inflammatory bowel disease patients: analyses of two 8-year intercept cohorts. Inflamm Bowel Dis 2010;16:1541-9.

10. Roblin X, Oussalah A, Chevaux JB, Sparrow M, Peyrin-Biroulet L. Use of thiopurine testing in the management of inflammatory bowel diseases in clinical practice: a worldwide survey of experts. Inflamm Bowel Dis 2011;17:2480-7.

11. Newman WG, Payne K, Tricker K, Roberts SA, Fargher E, Pushpakom S, Alder JE, Sidgwick GP, Payne D, Elliott RA, Heise M, Elles R, Ramsden SC, Andrews J, Houston JB, Qasim F, Shaffer J, Griffiths CE, Ray DW, Bruce I, Ollier WE; TARGET study recruitment team. A pragmatic randomized controlled trial of thiopurine methyltransferase genotyping prior to azathioprine treatment: the TARGET study. Pharmacogenomics 2011;12:815-26.

12. Sayani FA, Prosser C, Bailey RJ, Jacobs P, Fedorak RN. Thiopurine methyltransferase enzyme activity determination before treatment of inflammatory bowel disease with azathioprine: effect on cost and adverse events. Can J Gastroenterol 2005;19:147-51.

13. Derijks LJ, Wong DR. Pharmacogenetics of thiopurines in inflammatory bowel disease. Curr Pharm Des 2010;16:145-54.

14. Schaeffeler E, Fischer C, Brockmeier D, Wernet D, Moerike K, Eichelbaum M, Zanger UM, Schwab M. Comprehensive analysis of thiopurine S-methyltransferase phenotype-genotype correlation in a large population of German-Caucasians and identification of novel TPMT variants. Pharmacogenetics 2004; 14:407-17.

15. Swen JJ, Nijenhuis M, de Boer A, Grandia L, Maitland-van der Zee AH, Mulder H, Rongen GA, van Schaik $\mathrm{RH}$, Schalekamp T, Touw DJ, van der Weide J, Wilffert B, Deneer VH, Guchelaar HJ. Pharmacogenetics: from bench to byte--an update of guidelines. Clin Pharmacol Ther 2011;89:662-73.

16. Ford L, Graham V, Berg J. Whole-blood thiopurine S-methyltransferase activity with genotype concordance: a new, simplified phenotyping assay. Ann Clin Biochem 2006;43:354-60.

17. Lennard L, Singleton HJ. High-performance liquid chromatographic assay of human red blood cell thiopurine methyltransferase activity. J Chromatogr B Biomed Appl 1994;661:25-33.

18. Yates CR, Krynetski EY, Loennechen T, Fessing MY, Tai HL, Pui CH, Relling MV, Evans WE. Molecular diagnosis of thiopurine S-methyltransferase deficiency: genetic basis for azathioprine and mercaptopurine intolerance. Ann Intern Med 1997;126:608-14. 
19. Colombel JF, Ferrari N, Debuysere H, Marteau P, Gendre JP, Bonaz B, Soulé JC, Modigliani R, Touze Y, Catala P, Libersa C, Broly F. Genotypic analysis of thiopurine S-methyltransferase in patients with Crohn's disease and severe myelosuppression during azathioprine therapy. Gastroenterology. 2000; 118:1025-30.

20. Palmieri O, Latiano A, Bossa F, Vecchi M, D'Incà R, Guagnozzi D, Tonelli $F$, Cucchiara S, Valvano MR, Latiano T, Andriulli A, Annese V. Sequential evaluation of thiopurine methyltransferase, inosine triphosphate pyrophosphatase, and HPRT1 genes polymorphisms to explain thiopurines' toxicity and efficacy. Aliment Pharmacol Ther 2007;26:737-45.

21. Zabala-Fernández W, Barreiro-de Acosta M, Echarri A, Carpio D, Lorenzo A, Castro J, Martínez-Ares D, Pereira S, Martin-Granizo I, Corton M, Carracedo A, Barros F. A pharmacogenetics study of TPMT and ITPA genes detects a relationship with side effects and clinical response in patients with inflammatory bowel disease receiving Azathioprine. J Gastrointestin Liver Dis 2011;20:247-53.

22. Uchiyama K, Nakamura M, Kubota T, Yamane T, Fujise K, Tajiri H. Thiopurine S-methyltransferase and inosine triphosphate pyrophosphohydrolase genes in Japanese patients with inflammatory bowel disease in whom adverse drug reactions were induced by azathioprine/6-mercaptopurine treatment. J Gastroenterol 2009;44:197-203.

23. Zelinkova Z, Derijks L, Stokkers PC, Vogels EW, van Kampen AH, Curvers WL, Cohn D, van Deventer SJ, Hommes DW. Inosine triphosphate pyrophosphatase and thiopurine s-methyltransferase genotypes relationship to azathioprine-induced myelosuppression. Clin Gastroenterol Hepatol 2006;4:44-9.

24. Yang SK, Hong M, Baek J, Choi H, Zhao W, Jung Y, Haritunians T, Ye BD, Kim KJ, Park SH, Park SK, Yang DH, Dubinsky M, Lee I, McGovern DP, Liu J, Song K. A common missense variant in NUDT15 confers susceptibility to thiopurine-induced leukopenia. Nat Gen 2014;46:1017-1020.

25. van Asseldonk DP, Kanis BM, de Boer NK, van Bodegraven AA. Leukopenia due to parvovirus B19 in a Crohn's disease patient using azathioprine. Digestion 2009;79:211-4.

26. Nguyen TM, Le Gall C, Lachaux A, Boulieu R. High thiopurine metabolite concentrations associated with lymphopenia in inflammatory bowel disease (IBD) pediatric patients receiving aminosalicylates combined with azathioprine. Int J Clin Pharmacol Ther 2010;48:275-81.

27. Xin H, Fischer C, Schwab M, Klotz U. Effects of aminosalicylates on thiopurine S-methyltransferase activity: an ex vivo study in patients with inflammatory bowel disease. Aliment Pharmacol Ther 2005; 21:1105-9.

28. Cuffari C, Hunt S, Bayless T. Utilisation of erythrocyte 6-thioguanine metabolite levels to optimise azathioprine therapy in patients with inflammatory bowel disease. Gut 2001;48:642-6.

29. Cuffari C, Theoret Y, Latour S, Seidman G. 6-Mercaptopurine metabolism in Crohn's disease: correlation with efficacy and toxicity. Gut 1996;39:401-6.

30. Dubinsky MC, Lamothe S, Yang HY, Targan SR, Sinnett D, Théorêt Y, Seidman EG. Pharmacogenomics and metabolite measurement for 6-mercaptopurine therapy in inflammatory bowel disease. Gastroenterology 2000;118:705-13.

31. Connell WR, Kamm MA, Ritchie JK, Lennard-Jones JE. Bone marrow toxicity caused by azathioprine in inflammatory bowel disease: 27 years of experience. Gut 1993;34:1081-5.

32. Thompson AJ, Newman WG, Elliott RA, Roberts SA, Tricker K, Payne K. The cost-effectiveness of a pharmacogenetic test: a trial-based evaluation of TPMT genotyping for azathioprine. Value Health 2014;17:22-33.

33. Cosnes J, Gower-Rousseau C, Seksik P, Cortot A. Epidemiology and natural history of inflammatory bowel diseases. Gastroenterology 2011;140:1785-94. 



\section{Chapter 4}

The role of xanthine oxidase in thiopurine metabolism: a case report

DR Wong, LJ Derijks, MO den Dulk, EHKM Gemmeke, PM Hooymans Ther Drug Monit 2007;29:845-8 


\section{Abstract}

Azathioprine (AZA) is widely used in the treatment of autoimmune inflammatory diseases. AZA is normally rapidly and almost completely converted to 6-mercaptopurine (6-MP) in the liver, which is further metabolised into a variety of pharmacological active thiopurine metabolites. 6-MP is catabolised by xanthine oxidase (XO) to the inactive metabolite 6-thiouric acid (6-TUA). The authors report the case of a Caucasian woman with chronic autoimmune pancreatitis unable to form active thiopurine metabolites.

The 55-year-old woman presented with weight loss, progressive elevation of liver transaminases and serum amylase. She was treated with prednisolone $30 \mathrm{mg} 1 \mathrm{dd}(1 \mathrm{mg} / \mathrm{kg})$ and AZA was increased to $75 \mathrm{mg} 1 \mathrm{dd}(2.5 \mathrm{mg} / \mathrm{kg})$; later to $150 \mathrm{mg} 1 \mathrm{dd}(5 \mathrm{mg} / \mathrm{kg})$. Despite good patient compliance the active metabolites of AZA, 6-thioguanine nucleotides (6-TGN) and 6-methylmercaptopurine ribonucleotides (6-MMPR), could not be detected in the erythrocytes (RBC). Subsequently, AZA was switched to high-dose 6-MP $(2.5 \mathrm{mg} / \mathrm{kg})$ and the XO-inhibitor allopurinol was added. After one week this combination led to a high 6-TGN level of $616 \mathrm{pmol} /$ $8 \times 10^{8} \mathrm{RBC}$ and a 6 -MMPR level of $1,319 \mathrm{pmol} / 8 \times 10^{8} \mathrm{RBC}$. Three weeks after start, $6-\mathrm{TGN}$ and 6-MMPR even reached toxic levels $\left(1,163 \mathrm{pmol} / 8 \times 10^{8} \mathrm{RBC}\right.$ and $10,015 \mathrm{pmol} / 8 \times 10^{8} \mathrm{RBC}$, respectively), so that 6-MP treatment was discontinued. To elucidate this finding, 6-MP $(1.7 \mathrm{mg} / \mathrm{kg})$ was applied for three days without allopurinol. The woman was not able to form active thiopurine metabolites. According to the authors, this is the first report of a patient unable to form detectable active thiopurine metabolites on AZA and 6-MP therapy, despite good patient compliance. High XO activity led to an inability to form detectable levels of active thiopurine metabolites 6-TGN and 6-MMPR. This finding emphasizes the important role of XO in the biotransformation of thiopurines. 


\section{Introduction}

Azathioprine (AZA) is clinically used in regimens of organ transplantations and for immunosuppression in the treatment of chronic autoimmune inflammatory diseases. AZA is a thiopurine pro-drug and has no immunosuppressive activity. The pharmacological action is based on the formation of 6-mercaptopurine (6-MP) in the liver, which is metabolised into the pharmacological active 6-thioguanine nucleotides (6-TGN) and 6-methylmercaptopurine ribonucleotides (6-MMPR). ${ }^{1}$ We describe a case of a woman who is unable to form active thiopurine metabolites.

\section{Case report}

The 55-year-old Caucasian woman (30 kg, length $1.57 \mathrm{~m}$ ) with chronic autoimmune pancreatitis was unsuccessfully treated with AZA $50 \mathrm{mg} 1 \mathrm{dd}(1.7 \mathrm{mg} / \mathrm{kg})$ for six months. She presented with weight loss $(3 \mathrm{~kg})$, progressive elevation of liver transaminases and serum amylase. The woman was treated with $30 \mathrm{mg}$ prednisolone per day $(1 \mathrm{mg} / \mathrm{kg})$, which was tapered $5 \mathrm{mg}$ each week, and the AZA dosage was increased to $75 \mathrm{mg} 1 \mathrm{dd}$ $(2.5 \mathrm{mg} / \mathrm{kg})$. In this period, she was admitted several times for short-stays in the hospital. After five weeks no active thiopurine metabolites (6-TGN and 6-MMPR) were detected in erythrocytes, despite good patient compliance, also verified during hospital-stay. For this reason the AZA dose was increased to $150 \mathrm{mg}$ daily $(5 \mathrm{mg} / \mathrm{kg})$. Almost three weeks later, once again, none of the active metabolites of AZA were detected. 6-TGN and 6-MMPR levels in erythrocytes were measured using a HPLC assay previously reported by Derijks and colleagues. ${ }^{2}$ Subsequently, AZA was switched to high-dose 6-MP [75 mg/day $(2.5 \mathrm{mg} / \mathrm{kg})]$ and $100 \mathrm{mg} /$ day allopurinol was added. After one week treatment with 6-MP and allopurinol, the 6-TGN and 6-MMPR levels were $616 \mathrm{pmol} / 8 \times 10^{8}$ red blood cells (RBC) and $1,319 \mathrm{pmol} / 8 \times 10^{8} \mathrm{RBC}$, respectively. Three weeks after start, the 6 -TGN level was $1,163 \mathrm{pmol} / 8 \times 10^{8} \mathrm{RBC}$ and the $6 \mathrm{MMPR}$ level $10,015 \mathrm{pmol} / 8 \times 10^{8} \mathrm{RBC}$. These levels of 6-TGN and 6-MMPR were above the upper limits of the therapeutic ranges (values 500 and 5,700 respectively). ${ }^{2,3}$ The woman developed leukopenia $\left(<4.0 \times 10^{9}\right.$ leukocytes/I) so that treatment with 6-MP and allopurinol was discontinued. A week later, the 6-TGN and 6-MMPR levels decreased to $686 \mathrm{pmol} / 8 \times 10^{8} \mathrm{RBC}$ and 4,027 pmol/ $8 \times 10^{8} \mathrm{RBC}$, respectively (Figure 4.1).

To clarify this finding, three months later, the woman agreed to take 6-MP for three days without allopurinol: day $125 \mathrm{mg} /$ day $(0.8 \mathrm{mg} / \mathrm{kg})$, and on days 2 and 3, $50 \mathrm{mg} /$ day $(1.7 \mathrm{mg} / \mathrm{kg})$. On day 4, 6-TGN and 6-MMPR could not be detected in erythrocytes. Genotyping of the enzyme thiopurine S-methyltransferase (TPMT) revealed a wild-type $\left.\operatorname{TPMT}^{H / H}\left({ }^{*} 1\right)^{*} 1\right)$ genotype; the woman is an extensive metaboliser of thiopurines. 


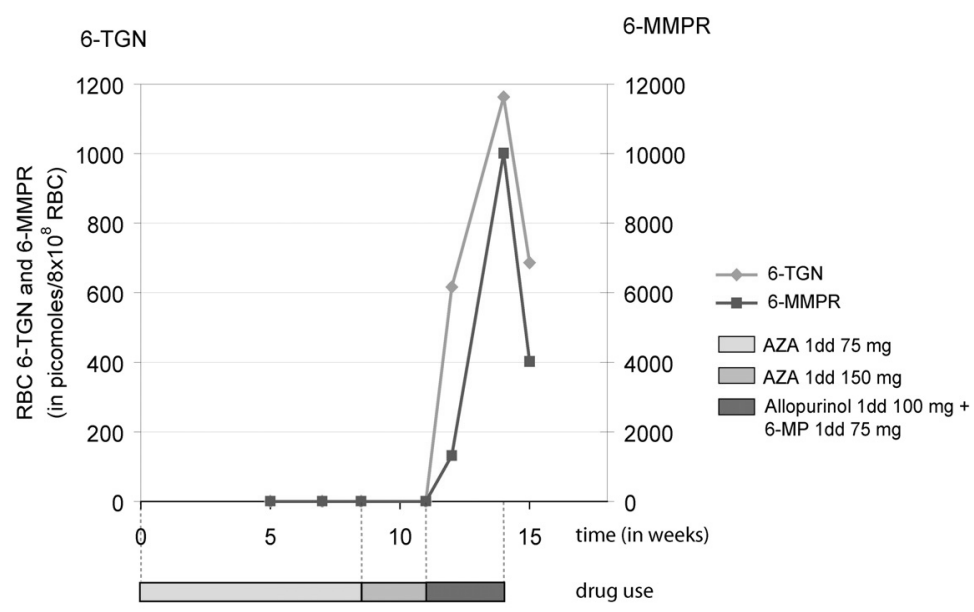

Figure 4.1 Thiopurine use and 6-TGN / 6-MMPR levels in time.

\section{Discussion}

To our knowledge this is the first report of a patient who was not able to form detectable active thiopurine metabolites, even on the treatment with a high dose AZA of $5 \mathrm{mg} / \mathrm{kg} /$ day.

As mentioned before, the pharmacological action of AZA is based on the formation of 6-MP in the liver, which is effected by elimination of the imidazole moiety.

This conversion is catalysed by the enzyme glutathione S-transferase (GST). ${ }^{4}$ Eklund et al. studied the catalytic activities of the numerous human GSTs in the biotransformation of AZA. This study showed that three different GSTs, A1-1, A2-2 and M1-1, expressed at high levels in the liver and small intestine, are the main enzymes that mediate the release of 6-MP from AZA by the nucleophilic substitution involving glutathione. The uncatalysed reaction of azathioprine with glutathione was estimated to be less than $1 \%$ of GST-catalysed biotransformation. Because important interindividual differences in the expression of human GSTs have been discovered, the authors concluded that in view of the predominance of GST-catalysed bio-activation of AZA over the non-enzymatic reaction with glutathione, individual differences in GST expression will affect the release of 6-MP and the formation of its metabolites. The polymorphism of GST M1-1 could be especially highly influential to the metabolism of AZA. ${ }^{4}$ A polymorphism of one of these GST-subtypes could result in low (or no) formation of 6-MP from AZA. 
The fact that the woman did not form detectable 6-TGN and 6-MMPR metabolites on exclusively 6-MP indicates that polymorphisms in the gene encoding GST cannot explain our finding.

The transformation of 6-MP into the various metabolites occurs along three competing metabolic routes: two catabolic by TPMT or xanthine-oxidase (XO) and one anabolic by hypoxanthine phosphoribosyl transferase (HPRT) (Figure 4.2).

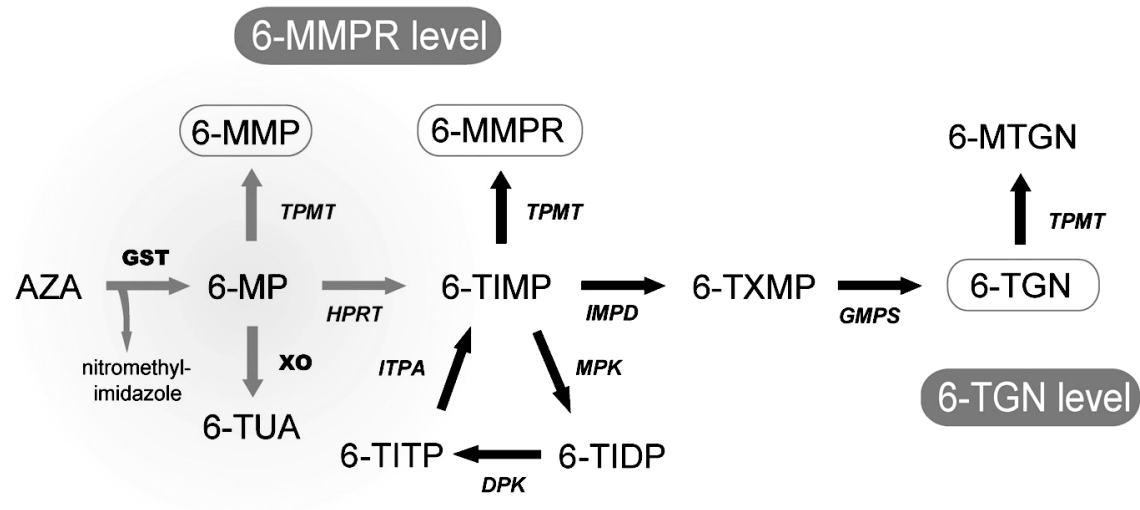

Figure 4.2 Proposed thiopurine metabolism.

AZA, azathioprine; 6-MP, 6-mercaptopurine; 6-MMP, 6-methylmercaptopurine; 6-TUA, 6-thiouric acid; 6-TIMP, 6-thioinosine monophosphate; 6-TXMP, 6-thioxanthosine monophosphate; 6-TIDP, 6-thioinosine diphosphate; 6-TITP, 6-thioinosine triphosphate; 6-MMPR, 6-methylmercaptopurine ribonucleotides; 6-TGN, 6-thioguaninenucleotides; 6-MTGN, 6-methylthioguaninenucleotides. GST, glutathione S-transferase; XO, xanthine oxidase; TPMT, thiopurine S-methyltransferase; HPRT, hypoxanthine phosphoribosyl transferase; IMPD, inosine monophosphate dehydrogenase; GMPS, guanosine monophosphate synthetase; MPK, monophosphate kinase; ITPA, inosine triphosphate pyrophosphatase.

The enzyme TPMT methylates 6-MP to 6-MMP. Furthermore, TPMT enables methylation of other compounds in the metabolic pathway, which may result in additional potentially active or hepatotoxic 6-MMPR metabolites. TPMT activity exhibits high interindividual variation as a result of genetic polymorphism. The frequency distribution of TPMT activity in Caucasian population is trimodal: one in 300 subjects have undetectable activity, $11 \%$ have intermediate activity, and approximately $89 \%$ of the population has high enzyme activity. An inverse relationship was demonstrated between erythrocyte TPMT activity and 6-TGN levels. TPMT determines the balance between 6-MMPR and 6-TGN. The higher the activity of TPMT, the less 6-MP is available for transformation to active 6-TGN. ${ }^{1}$

6-TGN levels are considered therapeutic above 235 to $250 \mathrm{pmol} / 8 \times 10^{8} \mathrm{RBC}^{3,5} 6-\mathrm{MMPR}$ levels in concentrations above $5,700 \mathrm{pmol} / 8 \times 10^{8} \mathrm{RBC}$ may lead to hepatotoxicity. ${ }^{3}$ 
In our case, TPMT genotyping revealed a wild-type $\left.\operatorname{TPMT}^{\mathrm{H} / H}\left({ }^{*} 1\right)^{*} 1\right)$ genotype, which means that the woman is an extensive metaboliser of thiopurines. TPMT is not the cause of our finding, because variation in TPMT activity results in another ratio between 6-TGN and 6-MMPR levels and not in undetectable levels of both metabolites. HPRT carries out the first anabolic step to produce 6-thioinosine monophosphate (6-TIMP), which subsequently is transformed into a variety of thiopurine nucleotide metabolites. ${ }^{1}$ There is only limited information on the range of HPRT activities in the healthy population. HPRT is completely deficient in people with the very rare LeschNyhan syndrome with characteristic symptoms like hyperuricaemia, spasticity, selfmutilation and developmental retardation. Since HPRT catalyses the conversion of 6-MP into 6-TIMP, there will not be an immunosuppressive effect of AZA or 6-MP in HPRT deficient patient. ${ }^{6}$ HPRT is partial deficient in people with the Kelley-Seegmiller syndrome, and can also give rise to a wide range of neurological symptoms and renal insufficiency. ${ }^{7}$ The characteristic symptoms of a (partial) HPRT deficiency were missing in our case. Moreover, since 6-MP in combination with allopurinol led to high 6-TGN and 6-MMPR levels in erythrocytes, demonstrates the woman is not HPRT-deficient.

$\mathrm{XO}$ is involved in the oxidation of endogenous purines and pyrimidines as well as thiopurines. In humans, the highest activities of $X O$ are found in the intestine and the liver; most other tissues show low activity. ${ }^{8}$ Differences in the activity of XO have implications with regard to individual variation in the biotransformation of 6-MP, contributing to a large patient variation in the bioavailability of 6-MP after an oral dose. Individual differences in XO enzyme activity could be determined either by genetic or other factors, such as dietary habits and inflammatory diseases. ${ }^{8-12}$

Competitive inhibition of XO with allopurinol $(300 \mathrm{mg} /$ day) pre-treatment results in a five-fold increase of the bioavailability of oral 6-MP. ${ }^{13}$ Raised erythrocyte 6-TGN levels have been demonstrated in patients receiving allopurinol. ${ }^{14}$ Furthermore, dangerous myelotoxicity resulting from concurrent use of allopurinol and 6-MP/AZA has been reported. $^{15,16}$

For concurrent use of allopurinol and 6-MP or AZA, it is recommended to reduce 6-MP/AZA dosage to a one-third or less of normal. ${ }^{13}$ Generally, approximately twothirds of a normal dose seems to be inactivated by the catabolic pathway of XO. Interestingly, Sparrow et al. investigated the effect of $100 \mathrm{mg} /$ day allopurinol in thiopurine non-responders with normal TPMT-activity and preferential shunting to 6-MMP. A reduction to $25 \%$ to $50 \%$ of the original 6-MP/AZA dose in combination with allopurinol led to an effective shift toward 6-TGN. ${ }^{17}$

In our case, erythrocyte 6-TGN and 6-MMPR levels were not detectable after high-dose AZA ( $5 \mathrm{mg} / \mathrm{kg}$ per day). However, an equivalent dose of $6-\mathrm{MP}(2.5 \mathrm{mg} / \mathrm{kg}$ per day) in combination with XO-inhibitor allopurinol resulted in a myelotoxic 6-TGN level in the first week after the start of treatment. Apparently, the addition of allopurinol inhibited the extensive conversion of 6-MP to 6-thiouric acid (6-TUA) catalysed by XO, resulting in 6-MP available for the formation of 6-TGN and 6-MMPR. Patient non-compliance during treatment of AZA and 6-MP in combination with allopurinol was ruled out during 
hospitalisation. Therefore, in our patient, a relatively high $\mathrm{XO}$ enzyme activity seems to be the only plausible explanation for our finding. To elucidate our finding, we applied 6-MP without allopurinol for three days. 6-TGN and 6-MMPR are normally detected already after three days, as shown in the pharmacokinetic analysis of 6-MP by Derijks and collegues. ${ }^{2}$ The fact that we could not detect 6-TGN and 6-MMPR levels on day 4 after three days of treatment with 6-MP (average $1.3 \mathrm{mg} / \mathrm{kg}$ per day) without allopurinol confirms our hypothesis.

\section{Conclusion}

To our knowledge, this is the first report of a patient who is not able to form detectable active thiopurine metabolites on the treatment with a therapeutic dose of AZA and 6-MP, despite good patient compliance. In daily practice, patients with low or zero levels are mostly considered non-compliant. However, our finding indicates that high $\mathrm{XO}$ activity could lead to a disability to form active thiopurine metabolites. In the past decade, much attention has been paid to the role of TPMT in thiopurine metabolism. This case emphasizes the importance of $\mathrm{XO}$ in the biotransformation of thiopurines in which high $\mathrm{XO}$ activity resulted in non-detectable levels of the active thiopurine metabolites 6-TGN and 6-MMPR. 


\section{References}

1. Lennard L. The Clinical pharmacology of 6-mercaptopurine. Eur J Clin Pharmacol 1992;43:329-39.

2. Derijks LJ, Gilissen LP, Engels LG, Bos LP, Bus PJ, Lohman JJ, Curvers WL, Van Deventer SJ, Hommes DW, Hooymans PM. Pharmacokinetics of 6-mercaptopurine in patients with inflammatory bowel disease: implications for therapy. Ther Drug Monit 2004;26:311-8.

3. Dubinsky MC, Lamothe S, Yang HY, Targan SR, Sinnett D, Théorêt Y, Seidman EG. Pharmacogenomics and metabolite measurement for 6-mercaptopurine therapy in inflammatory bowel disease. Gastroenterology 2000;118:705-713.

4. Eklund $\mathrm{BI}$, Moberg M, Bergquist J, Mannervik B. Divergent activities of human glutathione transferases in the bioactivation of azathioprine. Mol Pharmacol 2006;70:747-54.

5. Cuffari C, Hunt S, Bayless TM. Enhanced bioavailability of azathioprine compared to 6-mercaptopurine therapy in inflammatory bowel disease: correlation with treatment efficacy; Aliment Pharmacol Ther 2000;14:1009-14.

6. Nyhan WL. The recognition of Lesch-Nyhan syndrome as an inborn error of purine metabolism. J Inher Metab Dis 1997;20:171-8.

7. Mateos FA, Puig JG. Purine metabolism in Lesch-Nyhan syndrome versus Kelley-Seegmiller syndrome. J Inherit Metab Dis 1994;17:138-42.

8. Pritsos CA. Cellular distribution, metabolism, and regulation of the xanthine oxidoreductase enzyme system. Chem Biol Interact 2000;129:195-208.

9. Aklillu E, Carrillo JA, Makonnen E, Bertilsson L, Ingelman-Sundberg M. Xanthine oxidase activity is influenced by environmental factors in Ethiopians. Eur J Clin Pharmacol 2003;59:533-536.

10. Guerciolini R, Szumlanski C, Weinshilboum RM. Human liver xanthine oxidase: nature and extent of individual variation. Clin Pharmacol Ther 1991;50:663-72.

11. Relling MV, Lin JS, Ayers GD, Evans WE. Racial and gender differences in N-acetyltransferase, xanthine oxidase, and CYP1A2 activities. Clin Pharmacol Ther 1992;52:643-58.

12. Saruwatari J, Nakagawa K, Shindo J, Tajiri T, Fujieda M, Yamazaki H, Kamataki T, Ishizaki T. A population phenotyping study of three drug-metabolizing enzymes in Kyushu, Japan, with use of the caffeine test. Clin Pharmacol Ther 2002;72:200-8.

13. Zimm S, Collins JM, O'Neill D, Chabner BA, Poplack DG. Inhibition of first-pass metabolism in cancer chemotherapy: interaction of 6-mercaptopurine and allopurinol. Clin Pharmacol Ther 1983;34:810-7.

14. Chocair P, Duley J, Simmonds HA, Cameron JS, lanhez L, Arap S, Sabbaga E. Low-dose allopurinol plus azathioprine/cyclosporin/ prednisolone, a novel immunosuppressive regimen. Lancet 1993;342:83-4.

15. Kennedy DT, Hayney MS, Lake KD. Azathioprine and allopurinol: the price of an avoidable drug interaction; Ann Pharmacother 1996;30:951-4.

16. Venkat Raman V, Sharman VL, Lee HA. Azathioprine and allopurinol: a potentially dangerous combination. J Intern Med 1990;228:69-71.

17. Sparrow MP, Hande SA, Friedman S, Lim WC, Reddy SI, Cao D, Hanauer SB. Allopurinol safely and effectively optimizes tioguanine metabolites in inflammatory bowel disease patients not responding to azathioprine and mercaptopurine. Aliment Pharmacol Ther 2005;22:441-6. 


\section{Part II}

Therapeutic drug monitoring of thiopurine metabolites to optimise efficacy and safety in inflammatory bowel diseases 



\section{Chapter 5}

Therapeutic drug monitoring of thiopurine metabolites in adult thiopurine tolerant IBD patients on maintenance therapy

DR Wong*, LPL Gilissen*, LGJB Engels, J Bierau, JA Bakker, ADC Paulussen, MJ Romberg-Camps, A Stronkhorst, P Bus, LP Bos, PM Hooymans, RW Stockbrügger, C Neef, AAM Masclee J Crohns Colitis 2012;6:698-707

"Both authors contributed equally to the manuscript. 


\section{Abstract}

Background and aims

Therapeutic drug monitoring of active metabolites of thiopurines, azathioprine and 6-mercaptopurine, is relatively new. The proposed therapeutic threshold level of the active 6-thioguanine nucleotides (6-TGN) is $\geq 235 \mathrm{pmol} / 8 \times 10^{8}$ erythrocytes. The aim of this prospective cross-sectional study was to compare 6-TGN levels in adult thiopurine tolerant IBD patients with an exacerbation with those in remission, and to determine the therapeutic 6-TGN cut-off level.

\section{Methods}

Hundred IBD patients were included. Outcome measures were thiopurine metabolite levels, calculated therapeutic 6-TGN cut-off level, CDAI/CAI scores, thiopurine dose and TPMT enzyme activity.

\section{Results}

Forty-one patients had an exacerbation, 59 patients were in remission. In $17 \%$ of all patients 6-TGN levels were compatible with non-compliance. The median 6-TGN levels were not significantly different between the exacerbation and remission group (227 versus $263 \mathrm{pmol} / 8 \times 10^{8}$ erythrocytes, $\left.\mathrm{p}=0.29\right)$. The previous reported therapeutic 6 -TGN cut-off level of $235 \mathrm{pmol} / 8 \times 10^{8}$ erythrocytes was confirmed in this study. Twenty-six of the 41 patients $(63 \%)$ with active disease had 6-TGN levels below this threshold and 24 of 59 IBD patients (41\%) in clinical remission $(p=0.04)$.

\section{Conclusions}

Thiopurine non-compliance occurs frequently both in active and quiescent disease. 6-TGN levels below or above the therapeutic threshold are associated with a significant higher chance of IBD exacerbation and remission, respectively. These data support the role of therapeutic drug monitoring in thiopurine maintenance therapy in IBD to reveal non-compliance or underdosing, and can be used as a practical tool to optimise thiopurine therapy, especially in case of thiopurine non-response. 


\section{Introduction}

Immunosuppression with thiopurines such as, azathioprine (AZA) and 6-mercaptopurine (6-MP) has become standard in the maintenance therapy of inflammatory bowel disease (IBD) patients. Thiopurine metabolism in humans is complex due to involvement of various enzymes. Neither AZA nor 6-MP has intrinsic activity, hence both drugs have to undergo extensive metabolic transformations, yielding to a variety of pharmacological active metabolites, of which the 6-thioguanine nucleotides (6-TGN) and 6-methylmercaptopurine ribonucleotides (6-MMPR) are considered to be the most important (Figure 5.1). ${ }^{1}$

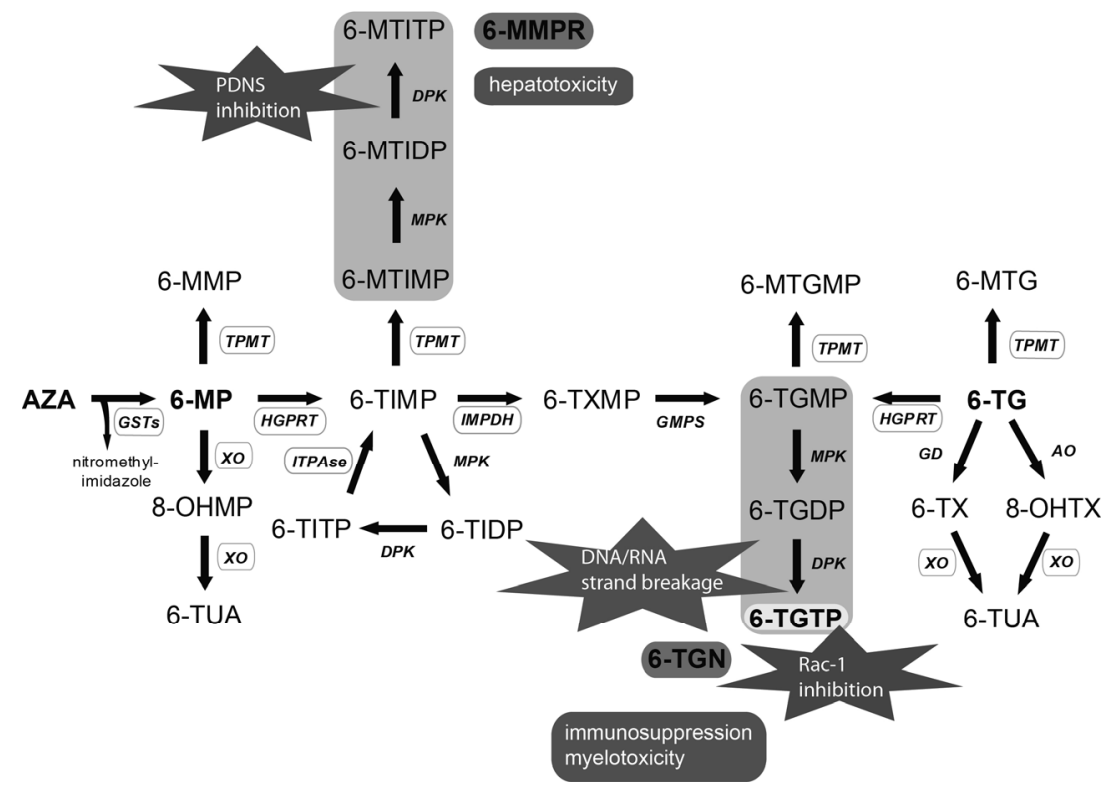

Figure 5.1 Proposed thiopurine metabolism.

AZA, azathioprine; 6-MP, mercaptopurine; 6-MMP, 6-methylmercaptopurine; 8-OHMP, 8-hydroxy-6-mercaptopurine; 6-TUA, 6-thiouric acid; 6-MTIMP, 6-methylthioinosine monophosphate; 6-MTIDP, 6-methylthioinosine diphosphate; 6-MTITP, 6-methylthioinosine triphosphate; 6-TIMP, 6-thioinosine monophosphate; 6-TIDP, 6-thioinosine diphosphate; 6-TITP, 6-thioinosine triphosphate; 6-TXMP, 6-thioxanthosine monophosphate; 6-TGMP, 6-thioguanine monophosphate; 6-TGDP, 6-thioguanine diphosphate; 6-TGTP, 6-thioguanine triphosphate; 6-MTGMP, 6-methylthioguanine monophosphate; 6-TG, 6-thioguanine; 6-MTG, 6-methyl-thioguanine; $\mathrm{XO}$, xanthine oxidase; TPMT, thiopurine S-methyl transferase; HGPRT, hypoxanthine-guanine phosphoribosyl transferase; IMPDH, inosine monophosphate dehydrogenase; GMPS, guanosine monophosphate synthetase; MPK, monophosphate kinase; DPK, diphosphate kinase; ITPase, inosine triphosphate pyrophosphatase; 6-TX, 6-thioxanthine; 8-OHTX, 8-hydroxy-6-thioxanthine; GD, guanine deaminase; AO, aldehyde oxidase. PDNS, purine de novo synthesis; DNA, deoxyribonucleic acid; RNA, ribonucleic acid. 6-MTIMP, 6-MTIDP and 6-MTITP together form the 6-methyl- mercaptopurine ribonucleotides (6-MMPR). 6-TGMP, 6-TGDP and 6-TGTP form together the 6-thioguanine nucleotides (6-TGN). ${ }^{1}$ 
Thiopurine S-methyltransferase (TPMT) geno- or phenotyping prior to thiopurine therapy may help to recognize TPMT polymorphism and prevent severe myelotoxicity. $2,3,4$ Therapeutic drug monitoring (TDM) of the active thiopurine metabolites 6-TGN and 6-MMPR is a neglected, but attractive option that may help to optimise drug therapy. ${ }^{5-7}$ In IBD patients with an exacerbation while on thiopurine maintenance therapy, a switch to biologicals is usually made without further attempt to optimise thiopurine dosage based on thiopurine metabolite levels.

In recent years, therapeutic ranges of thiopurine metabolite concentrations have been described by several groups: 6-TGN metabolites between 235 and $490 \mathrm{pmol} / 8 \times 10^{8}$ red blood cells $(\mathrm{RBC})$ are associated with clinical response. ${ }^{5,8}$ The risk for leukopenia increases with 6-TGN levels above $490 \mathrm{pmol} / 8 \times 10^{8} \mathrm{RBC}$, whereas 6-MMPR levels higher than $5,700 \mathrm{pmol} / 8 \times 10^{8} \mathrm{RBC}$ are associated with hepatotoxicity. ${ }^{5,6}$ A 6-MMPR/6-TGN ratio higher than 11 is correlated with a lower frequency of clinical response. ${ }^{6,9}$

Although these studies found no correlation between metabolite levels and drug dose resulting from inter-individual differences in metabolism, in daily clinical practice thiopurine drugs have still been dosed based on the patient's bodyweight (AZA 2-3 $\mathrm{mg} / \mathrm{kg}$ and 6-MP 1-1.5 mg/kg). ${ }^{10}$

Osterman and colleagues performed a meta-analysis of six studies and showed that higher 6-TGN levels are correlated with clinical remission of IBD: $62 \%$ of the patients with 6-TGN levels above $230-260 \mathrm{pmol} / 8 \times 10^{8} \mathrm{RBC}$ were in remission versus $36 \%$ with 6 -TGN levels lower than $230 \mathrm{pmol} / 8 \times 10^{8} \mathrm{RBC}$, with a pooled odds ratio of $3.3(95 \% \mathrm{Cl}$ : 1.71-6.27; $\mathrm{p}<0.001) .{ }^{18}$ Most of the data on 6 -TGN metabolite therapeutic thresholds have been obtained in smaller subsets of IBD patients, in the early phase after initiating thiopurine therapy or in paediatric IBD cohorts. ${ }^{5,6,9,11-19}$

The present study was undertaken to compare thiopurine metabolite levels in a larger group of adult thiopurine tolerant IBD patients on maintenance therapy with an exacerbation and those in clinical remission, in order to be able to find the optimal threshold 6-TGN level for therapeutic efficacy. Such parameters may help to determine the role of TDM in clinical decision making: either to optimise thiopurine dosage, to avoid toxicity or to switch to an alternative treatment.

\section{Materials and methods}

\section{Patient selection}

A prospective cross-sectional study was performed in a group of IBD patients in four hospitals in the southern part of the Netherlands: one university hospital (Maastricht University Medical Centre) and three general district hospitals (Maasland Hospital Sittard (since 2009: Orbis Medical Centre), Laurentius Hospital Roermond and Catharina Hospital Eindhoven). Adult IBD patients, presenting an exacerbation while on tolerated maintenance dose of AZA or 6-MP for at least 3 months, were asked to participate. 
Meanwhile, consecutive IBD patients in clinical remission while on maintenance AZA or 6-MP for at least 3 months were also asked to participate. Concomitant IBD medication had to be stable for at least 3 months and was registered, including medication known or suspected to interfere with thiopurine metabolism in vivo (i.e. mesalazine and infliximab). ${ }^{20,21}$

At inclusion, the following parameters were assessed: haemoglobin count, haematocrit, leukocytes, thrombocytes, bilirubine, aspartate transaminase, amylase, C-reactive protein and serum albumin. Also, blood samples were collected for 6-TGN and 6-MMPR metabolite levels and TPMT activity measurement. Disease activity was scored by the Crohn's Disease Activity Index (CDAI) or Colitis Activity Index (CAI). ${ }^{22}$ Written informed consent was obtained from all participating patients.

\section{Outcome parameters}

Primary outcome parameters were 6-TGN and 6-MMPR metabolite levels, to be used for correlations with CDAI or CAI scores and the calculation of a therapeutic 6-TGN threshold level.

Zero metabolite levels were defined as non-detectable 6-TGN and 6-MMPR levels, and were considered to indicate non-compliance. Very low levels, defined as combined 6 -TGN levels $<150 \mathrm{pmol} / 8 \times 10^{8} \mathrm{RBC}$ and 6 -MMPR levels $<900 \mathrm{pmol} / 8 \times 10^{8} \mathrm{RBC}$, without diminished TPMT activity, were considered indicative for non-compliance or low compliance.

An exacerbation was defined by a CDAl score $\geq 200$ for Crohn's disease patients or a CAI score $\geq 8$ for patients with ulcerative colitis (UC) or indeterminate colitis.

Remission was defined by a CDAl score $<150$ or CAI $<8$. Patients with CDAl scores between 150 and 200 were not included to more clearly separate exacerbations or active disease from clinical remissions.

Secondary outcome parameters were CDAI and CAI scores, TPMT activity, 6-MMPR/6-TGN ratio and correlations between 6-TGN and 6-MMPR metabolite levels.

\section{Analytical procedures}

6-TGN and 6-MMPR levels were measured with the modified high performance liquid chromatography (HPLC) method of Lennard et al. as published previously. ${ }^{5,6,8,23}$

Blood samples were immediately stored in the refrigerator $\left(2-8^{\circ} \mathrm{C}\right)$ and subsequently sent to the laboratory of the Department of Clinical Pharmacy \& Toxicology of the Maasland Hospital (Sittard, The Netherlands) where the samples were stored at $-20^{\circ} \mathrm{C}$, so that stability of the metabolites was assured until measurement occurred.

The lower limit of quantification of the assay was determined at $30 \mathrm{pmol} / 8 \times 10^{8} \mathrm{RBC}$ for 6-TGN and $300 \mathrm{pmol} / 8 \times 10^{8} \mathrm{RBC}$ for 6-MMPR. ${ }^{6}$

TPMT enzyme activity was measured in erythrocyte lysates essentially as described by Jacques-Aigrain, ${ }^{24}$ using reversed phase HPLC for quantification. The inter-assay covariation of the procedure was less than $10 \%$, the typical recovery was $99 \%$. 
TPMT genotyping was performed using DNA extracted from whole blood using the Qiagen FlexiGene DNA kit (Qiagen, Venlo, The Netherlands) and the automated DNA isolation robot Autogenflex 3000 (Westburg, Leusden, The Netherlands). Exons 5, 7 and 10 of the TPMT gene and flanking intronic regions were screened by direct sequencing in order to detect the most prevalent Caucasian functional TPMT polymorphisms $A 80 P$ (*2), A154T (*3A/B) and $Y 240 C\left({ }^{*} 3 B / C\right)$, although any other known or unknown variant in these exons would also be detected. Sequences were aligned with TPMT mRNA NCBI reference sequence NM_000367.

\section{Statistical analysis}

Normality was tested by the Kolmogorov-Smirnov test. Data are expressed as means with $95 \%$ confidence interval $(95 \% \mathrm{Cl})$ or ranges. Pearson's correlation test was used for correlations. The frequency distributions of the different variables between the patients with an exacerbation and a remission were compared by means of the likelihood ratio Chi-square test or, when expected counts were less than five, Fisher's Exact test. The $t$-test for independent samples was used to compare continuous variables; the Mann-Whitney test was used when the assumption of a normal distribution did not hold.

P-values $<0.05$ were considered statistically significant.

Power calculation was based on an analytical relevant 6-TGN level difference of $50 \mathrm{pmol} / 8 \times 10^{8} \mathrm{RBC}$ and a $2 \times$ standard deviation of $75 \mathrm{pmol} / 8 \times 10^{8} \mathrm{RBC}$, to exclude variation introduced by the HPLC method based on data of previous reports from our group. ${ }^{6,25}$ Based on these data 74 patients (at least 37 in each group) should be included to be able to demonstrate a significant difference in 6-TGN levels between both groups with a probability of $80 \%$. A total of 100 patients (preferably 50 patients per study group) was aimed at. To find a therapeutic threshold 6-TGN level, separate analyses were performed. Receiver Operating Characteristic curves of the 6-TGN levels have been plotted to find a 6-TGN cut-off level with an optimal sensitivity, specificity, positive predictive value and accuracy. At last, a quartile analysis was performed according to the original report of Dubinsky, to compare 6-TGN threshold levels. ${ }^{5}$

\section{Medical Ethics}

The study was approved by the local Medical Ethical Committee of the Maasland Hospital, Sittard, the Netherlands. 


\section{Results}

\section{Patients}

During 12 months, 123 IBD patients were asked to participate, see Figure 5.2. A total of 100 patients were included for primary analysis, 41 patients with IBD exacerbation and 59 patients with IBD in remission. Patient characteristics are given in Table 5.1.

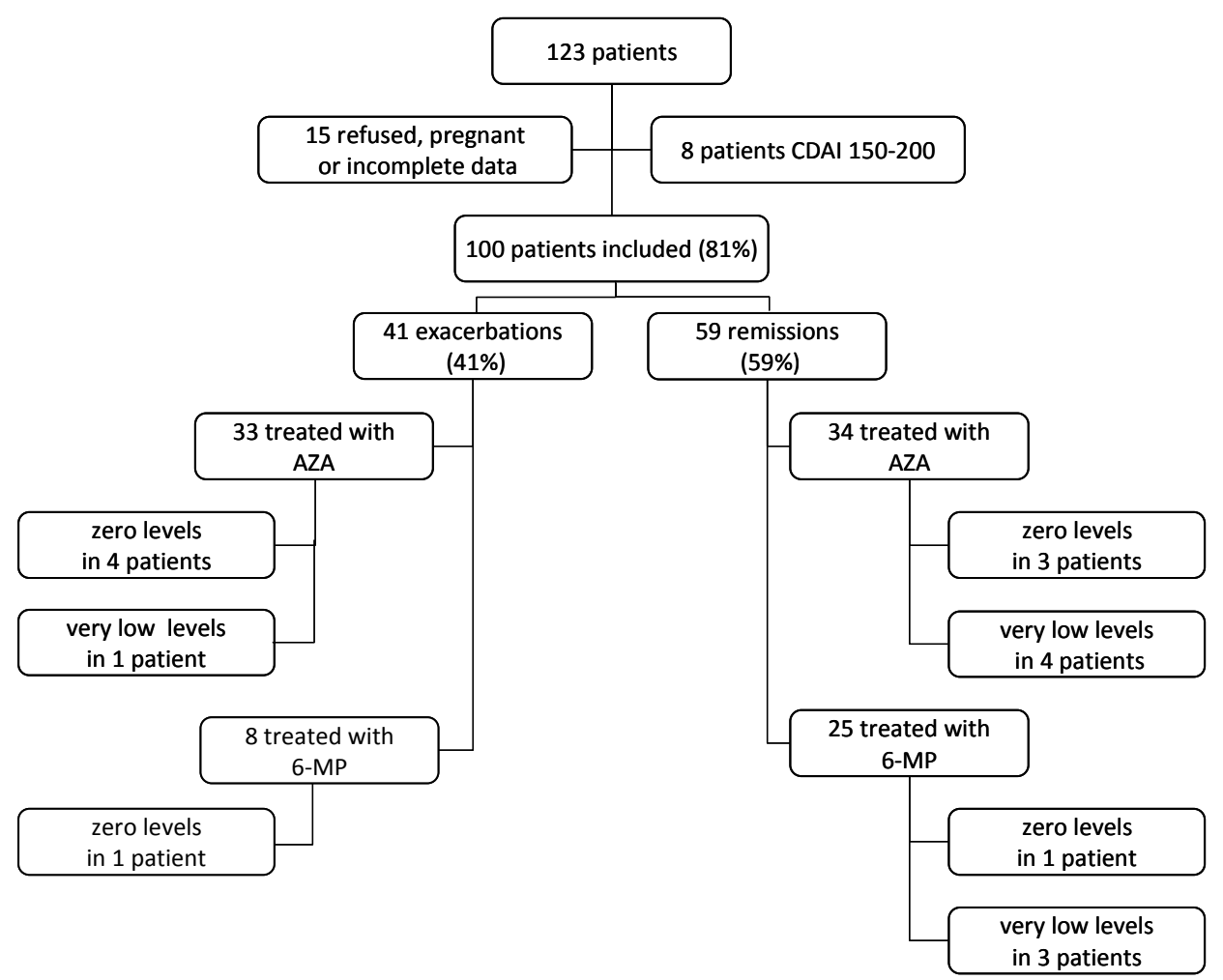

Figure 5.2 Flow chart of inclusion.

$\mathrm{CDAI}=$ Crohn's Disease Activity Index; $\mathrm{AZA}=$ azathioprine; 6-MP=6-mercaptopurine.

\section{Thiopurine metabolite levels}

No significant differences were found in drug doses or thiopurine metabolite levels between patients with Crohn's disease and ulcerative colitis. Therefore, in all further analyses Crohn's disease and UC patients were analysed as one group (IBD). Neither leukopenia, thrombocytopenia, disturbed liver enzymes nor elevated levels of serum amylase were observed in the IBD patients. 
Table 5.1 Characteristics of the patient population.

\begin{tabular}{|c|c|c|c|c|}
\hline & All patients & Exacerbation & Remission & p-value ${ }^{*}$ \\
\hline \multicolumn{5}{|l|}{ Patient } \\
\hline patient number: $\mathrm{n}$ & 100 & 41 & 59 & \\
\hline mean age in years (range) & $42.3(15-79)$ & $38.6(15-79)$ & $44.6(18-75)$ & 0.057 \\
\hline male/female & $60 / 40$ & $19 / 22$ & $41 / 18$ & 0.024 \\
\hline mean bodyweight (kg) & 75.4 & 73.7 & 76.5 & 0.34 \\
\hline CDAI (mean) & 157 & 281 & 72 & $<0.0001$ \\
\hline CAI (mean) & 6.8 & 11.9 & 3.1 & $<0.0001$ \\
\hline \multicolumn{5}{|l|}{ IBD } \\
\hline type & & & & 1.0 \\
\hline Crohn & 57 & 23 & 34 & \\
\hline ulcerative colitis & 40 & 16 & 24 & \\
\hline indeterminate colitis & 3 & 2 & 1 & \\
\hline \multicolumn{4}{|l|}{ Location } & 0.99 \\
\hline proctosigmoid & $35(35 \%)$ & $15(37 \%)$ & $20(34 \%)$ & \\
\hline colon & $24(24 \%)$ & $9(22 \%)$ & $16(27 \%)$ & \\
\hline ileocolon & $23(23 \%)$ & $11(27 \%)$ & $12(20 \%)$ & \\
\hline terminal ileum & $12(12 \%)$ & $5(12 \%)$ & $7(12 \%)$ & \\
\hline perianal disease & $7(7 \%)$ & $3(7 \%)$ & $4(7 \%)$ & \\
\hline proximal GI tract & $2(2 \%)$ & $1(2 \%)$ & $1(2 \%)$ & \\
\hline $\begin{array}{l}\text { Mean disease duration in months (range) } \\
\text { Thiopurine therapy }\end{array}$ & $98.7(3-624)$ & $99.9(3-432)$ & $97.9(5-624)$ & 0.93 \\
\hline Azathioprine (n) & 67 & $33(80 \%)$ & $34(58 \%)$ & 0.02 \\
\hline mean AZA dose in mg (range) & $140(50-225)$ & $136(50-200)$ & $145(50-225)$ & 0.33 \\
\hline mean AZA dose in mg/kg (range) & $1.8(0.6-3.0)$ & $1.9(0.8-2.5)$ & $1.8(0.6-3.0)$ & 0.75 \\
\hline 6-mercaptopurine (n) & 33 & $8(20 \%)$ & $25(42 \%)$ & 0.02 \\
\hline mean 6-MP dose in mg (range) & $56(50-100)$ & $56(50-100)$ & $56(50-100)$ & 0.96 \\
\hline mean 6-MP dose in mg/kg (range) & $0.8(0.5-1.2)$ & $0.8(0.6-1.2)$ & $0.8(0.4-1.2)$ & 0.99 \\
\hline Median thiopurine exposure in months (range) & $21.2(3.0-314)$ & $19.4(3.2-314)$ & $23.6(3.0-120)$ & 0.425 \\
\hline \multicolumn{5}{|l|}{ Concomitant IBD medication } \\
\hline oral mesalazine & 77 & $33(80 \%)$ & $44(75 \%)$ & 0.49 \\
\hline rectal mesalazine & 16 & $6(15 \%)$ & $10(17 \%)$ & 0.76 \\
\hline anti-TNF- $\alpha$ (i.e. infliximab) & 1 & $0(0 \%)$ & $1(2 \%)$ & 1.00 \\
\hline oral mesalazine + anti-TNF- $\alpha$ (i.e. infliximab) & 7 & $3(7 \%)$ & $4(7 \%)$ & 1.00 \\
\hline oral steroids (i.e. predniso(lo)ne) & 14 & $10(24 \%)$ & $4(7 \%)$ & 0.01 \\
\hline topical steroids (oral/rectal) & 32 & $14(34 \%)$ & $18(31 \%)$ & 0.70 \\
\hline
\end{tabular}

${ }^{*}$ p-value $<0.05$ is considered as statistically significant.

No significant differences were observed between IBD patients with exacerbation and IBD in remission concerning thiopurine dose, duration of thiopurine exposure, thiopurine metabolite levels, 6-MMPR/6-TGN ratio or TPMT activity. Data on thiopurine metabolites are given in Table 5.2.

The 6-TGN levels and TPMT activity were negatively correlated $(r=-0.318, p=0.01)$, although mean TPMT activity did not differ between the group with exacerbations and remissions. 
Non-detectable levels were found in 9 of all included patients (9\%). In 5 of 41 patients (12\%) with an exacerbation and in 4 of 59 patients (7\%) in remission no 6-TGN and 6-MMPR metabolite levels could be detected (Figure 5.2). Very low levels were found in 1 of 41 patients (2\%) with an exacerbation and 7 of 59 patients $(12 \%)$ in remission.

Based on non-detectable and very low metabolite levels, the (suspected) noncompliance rate for all 100 patients was $17 \%$. No significant difference was found in non-compliance between patients with an exacerbation and remission (15\% versus $19 \%$, Table 5.2). No correlations were found between 6-TGN levels and disease activity scores.

Based on the Receiver Operating Characteristic curve analysis the optimal 6-TGN cut-off level was $235 \mathrm{pmol} / 8 \times 10^{8} \mathrm{RBC}$ with a positive predictive value of $52 \%$ for exacerbations with 6-TGN levels below $235 \mathrm{pmol} / 8 \times 10^{8} \mathrm{RBC}$ and a negative predictive value of $70 \%$ with 6-TGN levels above $235 \mathrm{pmol} / 8 \times 10^{8} \mathrm{RBC}$ in case of remissions (Table 5.3).

Twenty-six of the 41 patients with active disease (63\%) had 6-TGN levels below the therapeutic threshold of $235 \mathrm{pmol} / 8 \times 10^{8} \mathrm{RBC}$, and 24 of the 59 patients in clinical remission (41\%) had levels below this threshold $(p=0.04)$.

Table 5.2 Outcome measures of all included patients.

\begin{tabular}{|c|c|c|c|c|}
\hline Outcome measures & All patients & $\begin{array}{l}\text { Exacerbations } \\
(n=41)\end{array}$ & $\begin{array}{l}\text { Remissions } \\
(n=59)\end{array}$ & p-value \\
\hline 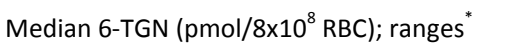 & $235(0-934)$ & $227(0-934)$ & $263(0-896)$ & 0.30 (n.s.) \\
\hline Median 6-MMPR (pmol/ $\left.8 \times 10^{8} \mathrm{RBC}\right)$; ranges ${ }^{*}$ & $645(0-11,418)$ & $606(0-9,386)$ & $683(0-11,418)$ & 0.89 (n.s.) \\
\hline Median 6-MMPR/6-TGN ratio & $2.35(0-63)$ & $2.36(0-58)$ & $2.38(0-63)$ & 0.97 (n.s.) \\
\hline Number of non-compliant patients (\%) & $17(17)$ & $6(15)$ & $11(19)$ & 0.42 (n.s.) \\
\hline Mean TPMT activity (pmol/mg Hb/h) & 7.0 & 6.7 & 7.2 & 0.16 (n.s.) \\
\hline
\end{tabular}

$\mathrm{N}=$ number of patients; kg=kilograms bodyweight; $\mathrm{mg}=$ milligrams; 6-TGN=6-thioguanine nucleotides level; 6-MMPR=6-methylmercaptopurine ribonucleotides level; pmol=picomoles; $\mathrm{RBC}=\mathrm{red}$ blood cells; TPMT=thiopurine S-methyltransferase; $\mathrm{Hb}=$ haemoglobin; $\mathrm{hr}=$ hour. P-value $<0.05$ is considered as statistically significant. n.s. $=$ not significant. ${ }^{*}$ Both 6-TGN and 6-MMPR levels were normal distributed.

Regarding 6-TGN levels, $30 \%$ of the patients with 6-TGN levels above $235 \mathrm{pmol} / 8 \times 10^{8}$ RBC had an exacerbation versus $52 \%$ of the IBD patients with 6-TGN levels below $235 \mathrm{pmol} / 8 \times 10^{8} \mathrm{RBC}(\mathrm{p}=0.04$, Figure 5.3). The odds ratio $(\mathrm{OR})$ for having active disease in case of a 6-TGN level below $235 \mathrm{pmol} / 8 \times 10^{8} \mathrm{RBC}$ was 2.5 (95\% $\mathrm{Cl}$ : 1.1-5.8) for all included patients.

Excluding all non-compliant patients in both groups, also showed no significant differences in median 6-TGN levels $\left(230 \mathrm{pmol} / 8 \times 10^{8} \mathrm{RBC}\right.$ (0-934) versus $294 \mathrm{pmol} / 8 \times 10^{8} \mathrm{RBC}(0-896)$ for the exacerbation and remission group, respectively; $\mathrm{p}=0.15$ ). The optimal threshold 6-TGN level appeared to be $235 \mathrm{pmol} / 8 \times 10^{8} \mathrm{RBC}$ (Table 
5.3). The OR for having active disease in case of a 6-TGN level below the therapeutic threshold level of $235 \mathrm{pmol} / 8 \times 10^{8} \mathrm{RBC}$ in the compliant patients was $3.8(95 \% \mathrm{Cl}$ : 1.5-9.4).

Table 5.3 Cut-off threshold 6-TGN levels (pmol/ $8 \times 10^{8} \mathrm{RBC}$ ).

\begin{tabular}{|c|c|c|c|c|c|c|c|}
\hline $\begin{array}{l}\text { Cut-off } \\
\text { 6-TGN level }\end{array}$ & $\begin{array}{l}\text { Sensitivity } \\
\text { (\%) }\end{array}$ & $\begin{array}{l}\text { Specificity } \\
\text { (\%) }\end{array}$ & $\begin{array}{l}\text { Positive } \\
\text { predictive } \\
\text { value (\%) }\end{array}$ & $\begin{array}{l}\text { Negative } \\
\text { predictive } \\
\text { value (\%) }\end{array}$ & p-value & $\begin{array}{l}\text { Likelihood } \\
\text { ratio }\end{array}$ & $\begin{array}{l}\text { Odds ratio } \\
\text { (Cl95\%) }\end{array}$ \\
\hline \multicolumn{8}{|c|}{ IBD patients $(n=100)$} \\
\hline 100 & 12 & 88 & 42 & 59 & 1.0 & 1.0 & $1.0(0.3-1.5)$ \\
\hline 150 & 24 & 78 & 43 & 59 & 0.8 & 1.1 & $1.1(0.4-2.9)$ \\
\hline 200 & 39 & 69 & 47 & 62 & 0.4 & 1.3 & $1.5(0.6-3.4)$ \\
\hline 235 & 63 & 59 & 52 & 70 & 0.04 & 1.6 & $2.5(1.1-5.7)$ \\
\hline 250 & 71 & 54 & 52 & 73 & 0.02 & 1.5 & $2.9(1.6-6.7)$ \\
\hline 300 & 83 & 37 & 48 & 76 & 0.04 & 1.3 & $2.9(1.1-7.6)$ \\
\hline \multicolumn{8}{|c|}{ All compliant patients $(n=83)$} \\
\hline 100 & 0 & 98 & 0 & 57 & 1.0 & 0 & $0.02(0.04-11.0)$ \\
\hline 150 & 14 & 94 & 63 & 59 & 0.3 & 2.2 & $2.4(0.5-10.9)$ \\
\hline 200 & 31 & 85 & 61 & 62 & 0.1 & 2.1 & $2.6(0.9-7.5)$ \\
\hline 235 & 58 & 73 & 62 & 70 & 0.007 & 2.2 & $3.8(1.5-9.4)$ \\
\hline 250 & 67 & 67 & 60 & 73 & 0.004 & 2.0 & $4.0(1.6-10.0)$ \\
\hline 300 & 81 & 46 & 53 & 76 & 0.02 & 1.5 & $3.5(1.3-9.5)$ \\
\hline 350 & 83 & 27 & 46 & 68 & 0.3 & 1.1 & $1.9(0.6-5.5)$ \\
\hline
\end{tabular}

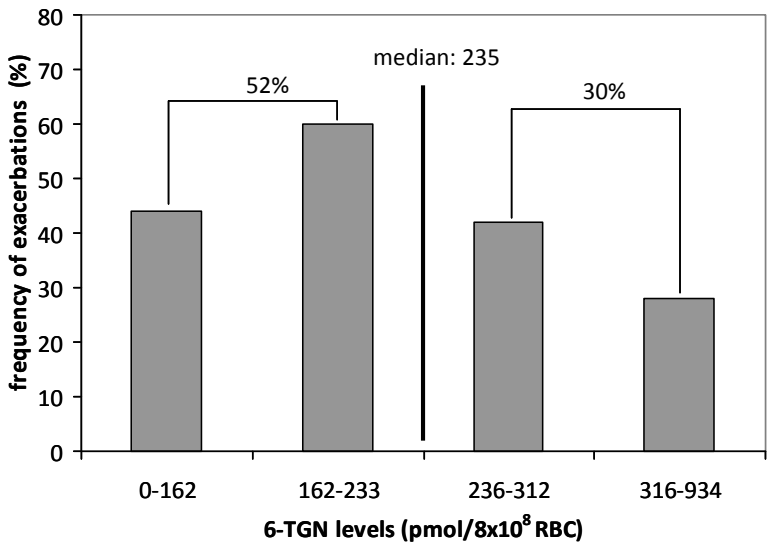

Figure 5.3 Quartile analysis of 6-TGN levels.

Exacerbation frequency of all patients is given for each 6-TGN level-quartile as performed by Dubinsky et al. ${ }^{5}$ Frequency (y-axis) was calculated by dividing the number of exacerbations by all patients in the specific 6-TGN level quartile. Thirty percent of all patients with 6-TGN levels $>235 \mathrm{pmol} / 8 \times 10^{8} \mathrm{RBC}$ had an exacerbation versus $52 \%$ of the IBD patients with 6 -TGN levels $<235 \mathrm{pmol} / 8 \times 10^{8} \mathrm{RBC}(\mathrm{p}=0.04)$. 


\section{TPMT activity and genotype}

High TPMT activity was found in 5 patients $(5 \%$, including one patient with an exacerbation): two of these patients (one exacerbation) demonstrated zero levels and one patient very low thiopurine metabolite levels, indicating non-compliance. In the other two patients therapeutic metabolite levels were found.

Low TPMT activity was found in 2 patients (2\%, 1 exacerbation). Both patients with low TPMT activity had high 6-TGN levels (712 and $934 \mathrm{pmol} / 8 \times 10^{8} \mathrm{RBC}$ ) and low 6-MMPR levels (both $<300 \mathrm{pmol} / 8 \times 10^{8} \mathrm{RBC}$ ), as expected. Low TPMT activity was not associated with myelotoxicity in this study.

TPMT genotyping revealed seven patients (7\%) with heterozygote TPMT ${ }^{*} 1 /{ }^{*} 3 A$ genotype. No homozygote mutant TPMT genotype was found in this cohort.

\section{Concomitant IBD medication}

No significant differences were found between both patient groups concerning the use of oral mesalazine, rectal mesalazine, and infliximab, the combined use of mesalazine and infliximab or topical steroid treatment (Table 5.1). A significant difference for oral steroid treatment was found between both IBD patient groups: $24 \%$ of the patients with an exacerbation and $7 \%$ of the patients with quiescent disease $(p=0.01)$ were using low-dose prednisolone.

\section{Discussion}

In this cross-sectional prospective study with IBD patients on thiopurine maintenance therapy we have shown that patients with a 6-TGN level above $235 \mathrm{pmol} / 8 \times 10^{8} \mathrm{RBC}$ have a significantly higher chance of being in remission compared to IBD patients with 6-TGN levels below $235 \mathrm{pmol} / 8 \times 10^{8} \mathrm{RBC}$.

No significant differences were found in median 6-TGN or 6-MMPR metabolite levels between IBD patients with exacerbation and remission. Neither a correlation was found between 6-TGN levels, 6-MMPR/6-TGN ratio and disease activity. In this respect, our data are consistent with conclusions of other reports on TDM of thiopurines. ${ }^{12,16-18}$

The defined therapeutic threshold 6-TGN level of $235 \mathrm{pmol} / 8 \times 10^{8} \mathrm{RBC}$ had poor sensitivity, specificity and positive predictive value of maximum $72 \%, 60 \%$ and $52 \%$ respectively, resulting in an accuracy of $67 \%$. The therapeutic threshold level we defined is similar to the proposed 6-TGN threshold of $235 \mathrm{pmol} / 8 \times 10^{8} \mathrm{RBC}$ in a study on paediatric IBD patients. ${ }^{5}$ Employing an analysis with quartiles, according to Dubinsky et al., ${ }^{5}$ revealed a corresponding therapeutic threshold 6-TGN level (Figure 5.3). In that study, median metabolite levels in IBD patients with exacerbations and remissions were significantly different (199 versus $312 \mathrm{pmol} / 8 \times 10^{8} \mathrm{RBC}$ ). ${ }^{5}$

We found a significant difference in the percentage of IBD patients with exacerbations and remissions with 6-TGN levels above the threshold of $235 \mathrm{pmol} / 8 \times 10^{8} \mathrm{RBC}: 37 \%$ of 
IBD patients with an exacerbation and $59 \%$ in remission. This finding is in accordance with the results of a recent meta-analysis performed by Osterman et al., describing $38 \%$ and $62 \%$ respectively. ${ }^{19}$

The OR for being in clinical remission in case of 6-TGN levels above the threshold was 2.5 for all included IBD patients and is in agreement with the results of the metaanalysis, in which an OR of 3.3 was found. ${ }^{19}$

As expected, non-compliance will negatively influence clinical efficacy of pharmacotherapy and is associated with long-term maintenance therapy of chronic diseases, such as diabetes mellitus and hypertension. In the population of IBD patients we studied zero or very low thiopurine metabolite levels suggested non-compliance in $17 \%$ of all patients, emphasizing that clinicians should always be well aware that nonadherence also frequently occurs in IBD. ${ }^{26-28}$

Interestingly, exclusion of all non-compliant patients in the present study increased the OR to 3.8 for being in clinical remission for patients with 6-TGN levels above the therapeutic threshold level of $235 \mathrm{pmol} / 8 \times 10^{8} \mathrm{RBC}$.

TDM is the only method to reveal non-compliance of thiopurine therapy, pointing to an important role for TDM in case of refractory IBD. Theoretically, zero or very low 6-TGN levels may result from other factors, such as thiopurine malabsorption or yet unknown enzyme defects or extremely high enzyme activity in the thiopurine metabolism pathway. Recently, a very high xanthine oxidase enzyme activity has been suggested to be the cause of zero 6-TGN and 6-MMPR levels in an individual who was treated with high-dose 6-MP. ${ }^{29}$ It should be noticed here that the thiopurine metabolic pathway is very complex and not yet fully discovered. ${ }^{1,29,30}$

Low TPMT activity was observed in $7 \%$ of the IBD patients, which is relatively low, compared with results from previous reports. ${ }^{4,5,9,31-34}$ This discrepancy may be a result of the fact that all patients in our study were on thiopurine maintenance therapy for at least 3 months, so that patients with early toxicity due to TPMT polymorphism already discontinued therapy. An expected negative correlation between 6-TGN levels and TPMT activity was found $(r=-0.318$ and $p=0.014)$ and the two patients with low TPMT activity both had zero 6-MMPR and high 6-TGN levels. This is in accordance with a recent publication by Kwan et al. ${ }^{34}$ High TPMT activity did not demonstrate higher 6-MMPR levels in our population $(n=5)$, although two patients demonstrated zero levels.

Some remarks should be made about our study design and patient selection. First, we included patients visiting the outpatient department during regularly planned visits. All patients were on thiopurine maintenance therapy for at least 3 months, thereby excluding intolerant patients with early adverse events or toxicity. The delayed onset of therapeutic response on thiopurines was taken into account, as clinical efficacy occurs in general after 3-4 months after initiation of thiopurine therapy. ${ }^{35}$ The obtained results are therefore only applicable to thiopurine tolerant IBD patients on maintenance therapy after at least three months. Second, patients with mild active Crohn's disease were not included, in order to be able to clearly differentiate exacerbations from 
remissions. The power analysis requested the inclusion of a substantial number of patients with active disease in order to draw conclusions on thiopurine metabolite levels in relation to disease activity. Studies that have included many patients with intermediate disease activity were not able to draw any conclusions with respect to TDM. $^{36,37}$ Third, the patient group was heterogenic with a broad spectrum of IBD phenotypes and two different disease activity scores have been used. Fourth, both AZA and 6-MP treated patients were included. AZA is a pro-drug of 6-MP (Figure 5.1). When using a conversion factor of 0.5, AZA dose is equivalent with 6-MP dose. Efficacy is considered equal for both drugs. ${ }^{10}$ No correlation was found between AZA/6-MP dose and 6-TGN metabolite levels, pointing to considerable interindividual variation in thiopurine metabolism. Fifth, the effect of other concomitant medication - contributing to therapeutic response and/or possibly interfering with thiopurine metabolism - was taken into account. We found no significant differences between both groups with regard to mesalazine use, anti-TNF- $\alpha$ and topical corticosteroids. However, patients with an exacerbation were more often treated with low-dose oral steroids than patients in remission. A poor clinical response to (thiopurine) therapy or thiopurine resistance may be a plausible explanation for this observation. Patients with active disease need (oral) steroid treatment in order to obtain or maintain clinical remission. Based on the 6-TGN threshold level of $235 \mathrm{pmol} / 8 \times 10^{8} \mathrm{RBC}$ significant differences were observed in the prevalence of IBD exacerbations and remissions in patients with 6-TGN levels above and below this threshold level, which confirms the clinical value of TDM, although sensitivity and specificity are rather poor. ${ }^{5,6,11,15}$

Our study was observational and not designed as interventional with dose adjustment based on thiopurine metabolite levels. For optimalisation of thiopurine therapy, TDM with dose adjustments should be undertaken.

Recently two studies have addressed this issue. The first one was performed by Reinshagen et al. who demonstrated that AZA dose-adjustment strategy based on therapeutic 6-TGN levels was not clinically superior to standard dosing with $2.5 \mathrm{mg} / \mathrm{kg}$ in patients with CD and normal TPMT activity: no significant differences were found concerning 6-TGN levels and disease activity at weeks 16 and $24 .^{38}$

The second study of González-Lama et al. was performed to predict safety and efficacy in steroid-resistant or -dependent IBD patients starting with thiopurine therapy. Early TPMT activity and 6-TGN level measurements were evaluated in thiopurine responders and non-responders until 6 months after steroid withdrawal. No proper 6-TGN threshold value to adjust thiopurine dose could be identified and it was demonstrated that early determination of TPMT activity and AZA metabolites were not useful to predict clinical response and thiopurine safety. ${ }^{39}$

Discontinuation of thiopurine therapy occurs regularly. Recently, it was shown in a retrospective study of an 8-year intercept cohort of thiopurine using IBD patients that about $25 \%$ discontinued thiopurine therapy within the first 3 months due to adverse events. The authors of that study showed that 6-TGN and 6-MMPR levels were not predictors for discontinuation of thiopurines, neither was the $6 \mathrm{MMPR} / 6-\mathrm{TGN}$ ratio. $^{40}$ 
On the other hand, TDM has proven to be helpful in toxicity preventing strategies and in dose-optimisation of patients with inadequate thiopurine efficacy. ${ }^{41,42}$

A recently performed worldwide survey pointed out that thiopurine testing by gastroenterologists in therapeutic strategies aimed at optimising thiopurine treatment is currently underutilised. ${ }^{43}$

Although present study was not designed to examine the efficacy of dose-optimising strategies based on thiopurine metabolite levels, it may be interesting to note that in only 4 of 26 patients (15\%) with active disease and subtherapeutic 6-TGN levels the thiopurine dose was increased to optimise IBD treatment successfully. In 2 of 6 patients who were additionally treated with anti-TNF therapy to achieve clinical remission, zero levels were found. These data were later retrospectively collected and show that in daily clinical practice apparently alternative therapies, such as systemic or topical steroids or expensive anti-TNF therapy, are preferred over dose-adjustment based on thiopurine metabolite levels. This again demonstrates that the therapeutic potential of thiopurines certainly has not been fully utilised.

In thiopurine optimising strategies many confounding factors are involved, like the large inter- and intra-individual variation of thiopurine metabolism (also during therapy), the slow therapeutic onset of thiopurines, the natural relapsing and remitting character of IBD, thiopurine resistance among IBD patients and the fact that thiopurine metabolite levels in erythrocytes actually are a surrogate marker for the target cells, the leukocytes. ${ }^{1,44,45}$

Therefore, to clarify the role of TDM in thiopurine optimising strategies there is need for larger long-term, prospective, randomised studies which follow patients on thiopurine treatment directly from the start of therapy, examining failure of treatment, efficacy and toxicity at several time points, comparing conventional follow up with dose-optimisation guided by TDM.

The clinical relevance of our study results is as follows: in an individual IBD patient with an exacerbation or therapy failure, measurement of 6-TGN metabolite levels should be undertaken to exclude non-compliance or underdosing. In case of non-compliance, the patient should be convinced to take thiopurines properly. When TDM shows 6-TGN levels $<235 \mathrm{pmol} / 8 \times 10^{8} \mathrm{RBC}$ and 6-MMPR $<<5,700 \mathrm{pmol} / 8 \times 10^{8} \mathrm{RBC}$, an attempt to dose adjustment should be undertaken. When TDM shows 6 -TGN levels $<235 \mathrm{pmol} / 8 \times 10^{8}$ $\mathrm{RBC}$ and 6-MMPR $>>5,700 \mathrm{pmol} / 8 \times 10^{8} \mathrm{RBC}$, a preferential 6-MMPR phenotype is revealed, possibly leading to poor response and hepatotoxicity. In these cases, the clinician may consider the addition of mesalazine or low-dose allopurinol (along with thiopurine dose reduction to $25-33 \%$ of the original dose) to shift thiopurine metabolism towards the active 6-TGN and optimise thiopurine efficacy. Also, a switch to the alternative thiopurine, 6 -thioguanine, should be considered. ${ }^{44}$

We conclude that TDM of thiopurine metabolites in a random IBD population reveals that non-compliance occurs frequently. IBD patients with sub-therapeutic 6-TGN levels have a significant higher chance of having active disease, whereas IBD patients with therapeutic 6-TGN levels have a significant higher chance of being in remission. These 
data support the role of TDM in thiopurine maintenance therapy in IBD to reveal noncompliance and underdosing and can be used as a practical tool to optimise thiopurine therapy, especially in case of thiopurine non-response. 


\section{References}

1. Derijks LJ, Wong DR. Pharmacogenetics of thiopurines in inflammatory bowel disease. Curr Pharm Des 2010;16:145-54

2. Weinshilboum RM, Sladek SL. Mercaptopurine pharmacogenetics: monogenic inheritance of erythrocyte thiopurine methyltransferase activity. Am J Hum Genet 1980;32:651-62.

3. Krynetski EY, Evans WE. Genetic polymorphism of thiopurine S-methyltransferase: molecular mechanisms and clinical importance. Pharmacology 2000; 61:136-46.

4. Lennard L. TPMT in the treatment of Crohn's disease with azathioprine. Gut 2002;51:143-6.

5. Dubinsky MC, Lamothe S, Yang HY, Targan SR, Sinnett D, Théorêt Y, Seidman EG. Pharmacogenomics and metabolite measurement for 6-mercaptopurine therapy in inflammatory bowel disease. Gastroenterology 2000;118:705-13.

6. Derijks LJJ, Gilissen LPL, Engels LGJB, Bos LP, Bus PJ, Lohman JJHM, Curvers WL, Van Deventer SJH, Hommes DW, Hooymans PM. Pharmacokinetics of 6-mercaptopurine in patients with inflammatory bowel disease; implications for therapy. Ther Drug Monit 2004;26:311-8.

7. Al Hadithy AF, de Boer NK, Derijks LJ, Escher JC, Mulder CJ, Brouwers JR. Thiopurines in inflammatory bowel disease: pharmacogenetics, therapeutic drug monitoring and clinical recommendations. Dig Liver Dis 2005;37:282-97.

8. Cuffari C, Théorêt Y, Latour S, Seidman G. 6-Mercaptopurine metabolism in Crohn's disease: correlation with efficacy and toxicity. Gut 1996;39:401-6.

9. Dubinsky MC, Yang H, Hassard PV, Seidman EG, Kam LY, Abreu MT, Targan SR, Vasiliauskas EA. 6-MP metabolite profiles provide a biochemical explanation for 6-MP resistance in patients with inflammatory bowel disease. Gastroenterology 2002;122:904-15.

10. Sandborn WJ. Rational dosing of azathioprine and 6-mercaptopurine. Gut 2001;48:591-2.

11. Achkar JP, Stevens T, Easley K, Brzezinski A, Seidner D, Lashner B. Indicators of clinical response to treatment with six-mercaptopurine or azathioprine in patients with inflammatory bowel disease. Inflamm Bowel Dis 2004;10:339-45.

12. Goldenberg BA, Rawsthorne P, Bernstein CN. The utility of 6-thioguanine metabolite levels in managing patients with inflammatory bowel disease. Am J Gastroenterol 2004;99:1744-8.

13. Hindorf U, Lyrenas E, Nilsson A, Schmiegelow K. Monitoring of long-term thiopurine therapy among adults with inflammatory bowel disease. Scand J Gastroenterol 2004;39:1105-12.

14. Wright S, Sanders DS, Lobo AJ, Lennard L. Clinical significance of azathioprine active metabolite concentrations in inflammatory bowel disease. Gut 2004;53:1123-8.

15. Cuffari C, Hunt S, Bayless T. Utilisation of erythrocyte 6-thioguanine metabolite levels to optimise azathioprine therapy in patients with inflammatory bowel disease. Gut 2001;48:642-6.

16. Belaiche J, Desager JP, Horsmans Y, Louis E. Therapeutic drug monitoring of azathioprine and 6-mercaptopurine metabolites in Crohn disease. Scand J Gastroenterol 2001;36:71-6.

17. Lowry PW, Franklin CL, Weaver AL, Pike MG, Mays DC, Tremaine WJ, Lipsky JJ, Sandborn WJ. Measurement of thiopurine methyltransferase activity and azathioprine metabolites in patients with inflammatory bowel disease. Gut 2001;49:665-70.

18. Gupta P, Gokhale R, Kirschner BS. 6-mercaptopurine metabolite levels in children with inflammatory bowel disease. J Pediatr Gastroenterol Nutr 2001;33:450-4.

19. Osterman MT, Kundu R, Lichtenstein GR, Lewis JD. Association of 6-thioguanine nucleotide levels and inflammatory bowel disease activity: a meta-analysis. Gastroenterology 2006;130:1047-53.

20. De Boer NK, Wong DR, Jharap B, de Graaf P, Hooymans PM, Mulder CJ, Rijmen F, Engels LG, van Bodegraven AA. Dose-dependent influence of 5-Aminosalicylates on thiopurine metabolism. Am J Gastroenterol 2007;102:1-7

21. Roblin X, Serre-Debeauvais F, Phelip J-M, Bessard G, Bonaz B. Drug interaction between infliximab and azathioprine in patients with Crohn's disease. Aliment Pharmacol Ther 2003;18:917-925

22. Lichtiger S, Present DH, Kornbluth A, Gelernt I, Bauer J, Galler G, Michelassi F, Hanauer S. Cyclosporine in severe ulcerative colitis refractory to steroid therapy. N Engl J Med 1994; 330:1841-5.

23. Lennard L, Singleton HJ. High-performance liquid chromatographic assay of the methyl and nucleotide metabolites of 6-mercaptopurine: quantitation of red blood cell 6-thioguanine nucleotide, 6-thioinosinic acid and 6-methylmercaptopurine metabolites in a single sample. J Chromatogr 1992;583:83-90. 
24. Jacques-Aigrain E, Bessa E, Medard Y, Mircheva Y, Vilmer E. Thiopurine methyltransferase activity in a French population: HPLC assay conditions and effects of drugs and inhibitors. Br J Clin Pharmacol 1994; 38:1-8.

25. Gilissen LP, Derijks L, Bos LP, Bus PJ, Hooymans PM, Engels LG. Therapeutic drug monitoring in 15 patients with inflammatory bowel disease and established azathioprine therapy. Clin Drug Invest 2004; 24:479-86.

26. Kane SV, Cohen RD, Aikens JE, Hanauer SB. Prevalence of nonadherence with maintenance mesalamine in quiescent ulcerative colitis. Am J Gastroenterol 2001;96:2929-33.

27. Sewitch MJ, Abrahamowicz M, Barkun A, Bitton A, Wild GE, Cohen A, Dobkin PL. Patient nonadherence to medication in inflammatory bowel disease. Am J Gastroenterol 2003;98:1535-44.

28. Kane SV, Huo D, Aikens JE, Hanauer SB. Medication nonadherence and the outcome of patients with quiescent ulcerative colitis. Am J Med 2003;114:39-43.

29. Wong DR, Derijks LJ, den Dulk MO, Gemmeke EHKM, Hooymans PM. The role of xanthine oxidase in thiopurine metabolism: a case report. Ther Drug Monit 2007;29:845-848

30. Gilissen LP, Derijks LJ, Verhoeven HM, Bierau J, Hooymans PM, Hommes DW, Engels LG. Pancytopenia due to high 6-methylmercaptopurine levels in a 6-mercaptopurine treated patient with Crohn's disease. Dig Liver Dis 2007;39:182-6.

31. Ansari A, Hassan C, Duley J, Marinaki A, Shobowale-Bakre EM, Seed P, Meenan J, Yim A, Sanderson J. Thiopurine methyltransferase activity and the use of azathioprine in inflammatory bowel disease. Aliment Pharmacol Ther 2002;16:1743-50.

32. Campbell S, Kingstone K, Ghosh S. Relevance of thiopurine methyltransferase activity in inflammatory bowel disease patients maintained on low-dose azathioprine. Aliment Pharmacol Ther 2002;16:389-98.

33. Regueiro M, Mardini H. Determination of thiopurine methyltransferase genotype or phenotype optimizes initial dosing of azathioprine for the treatment of Crohn's disease. J Clin Gastroenterology 2002;35:240-4.

34. Kwan LY, Devlin SM, Mirocha JM, Papadakis KA. Thiopurine methyltransferase activity combined with 6-thioguanine metabolite levels predicts clinical response to thiopurines in patients with inflammatory bowel disease. Dig Liver Dis 2008;40:425-32.

35. Pearson DC, May GR, Fick GH, Sutherland LR. Azathioprine and 6-mercaptopurine in Crohn disease. A meta-analysis. Ann Intern Med 1995;123:132-142

36. Kaskas BA, Louis E, Hindorf U, Schaeffeler E, Deflandre J, Graepler F, Schmiegelow K, Gregor M, Zanger UM, Eichelbaum M, Schwab M. Safe treatment of thiopurine S-methyltransferase deficient Crohn's disease patients with azathioprine. Gut 2003;52:140-2.

37. Mayer L. When is too much enough? Gastroenterology 2006;130:1352-4.

38. Reinshagen M, Schütz E, Armstrong VW, Behrens C, Von Tirpitz C, Stallmach A, Herfarth H, Stein J, Bias P, Adler G, Shipkova M, Kruis W, Oellerich M, Von Ahsen N. 6-thioguanine nucleotide-adapted azathioprine therapy does not lead to higher remission rates than standard therapy in chronic active crohn disease: results from a randomized, controlled, open trial. Clin Chem 2007;53:1306-14

39. González-Lama Y, Bermejo F, López-Sanromán A, García-Sánchez V, Esteve M, Cabriada JL, McNicholl AG, Pajares R, Casellas F, Merino O, Carpio D, Vera MI, Muñoz C, Calvo M, Benito LM, Bujanda L, GarcíaFernández FJ, Ricart E, Ginard D, Velasco M, Carneros JA, Manceñido N, Calvo M, Algaba A, Froilan C, Cara C, Maté J, Abreu L, Gisbert JP; "Grupo Español de Trabajo en Enfermedad de Crohn y Colitis Ulcerosa (GETECCU)". Thiopurine methyl-transferase activity and azathioprine metabolite concentrations do not predict clinical outcome in thiopurine-treated inflammatory bowel disease patients. Aliment Pharmacol Ther 2011;34:544-54

40. Jharap B, Seinen ML, De Boer NKH, Van Ginkel JR, Linskens RK, Kneppelhout JC, Mulder CJJ, Van Bodegraven AA. Thiopurine therapy in inflammatory bowel disease patients: analyses of two 8-year intercept cohorts Inflamm Bowel Dis 2010;16:1541-9.

41. Hanai H, lida T, Takeuchi K, Arai O, Watanabe F, Abe J, Maruyama Y, Oohata A, Ikeya K, Kageoka M, Miwa I, Yoshirou S, Hosoda Y, Kubota T. Thiopurine maintenance therapy for ulcerative colitis: the clinical significance of monitoring 6-thioguanine nucleotide. Inflamm Bowel Dis 2010;16:1376-81

42. Haines ML, Ajlouni Y, Irving PM, Sparrow MP, Rose R, Gearry RB, Gibson PR. Clinical usefulness of therapeutic drug monitoring in patients with inadequately controlled inflammatory bowel disease Inflamm Bowel Dis 2011;17:1301-7 
43. Roblin X, Oussalah A, Chevaux J-B, Sparrow M, Peyrin-Biroulet L. Use of Thiopurine Testing in the Management of Inflammatory Bowel Diseases in Clinical Practice: a Worldwide Survey of Experts. Inflamm Bowel Dis 2011;17:2480-7

44. Van Asseldonk DP, Sanderson J, de Boer NKH, Sparrow MP, Lémann M, Ansari A, Almer SH, Florin THJ, Gearry RB, Mulder CJ, Mantzaris G, van Bodegraven AA, Thiopurine Task Force Interest Group. Difficulties and possibilities with thiopurine therapy in inflammatory bowel disease-Proceedings of the first Thiopurine Task Force meeting. Dig Liver Dis 2011;43:270-6

45. Van Asseldonk DP, de Boer NKH, van Bodegraven AA. Thiopurine metabolite measurement - not for everyone. Aliment Pharmacol Ther 2011;34:1038-9 


\section{Chapter 6}

Early assessment of thiopurine metabolites identifies patients at risk of leukopenia in inflammatory bowel disease

DR Wong, MJH Coenen, SH Vermeulen, LJ Derijks, CJ van Marrewijk, OH Klungel, H Scheffer, B Franke, HJ Guchelaar, DJ de Jong, LGJB Engels, ALM Verbeek, PM Hooymans, on behalf of the TOPIC consortium

Submitted 


\section{Abstract}

Objective

Leukopenia is a potentially lethal adverse reaction of thiopurine treatment of inflammatory bowel disease (IBD). We determined the predictive value of the 6-thioguanine nucleotide (6-TGN) and 6-methylmercaptopurine ribonucleotide (6-MMPR) concentrations one week after initiation (T1) for the development of leukopenia during the first eight weeks of thiopurine treatment.

\section{Design}

The study was performed in IBD patients starting thiopurine therapy as part of the Dutch randomised controlled TOPIC trial (ClinicalTrials.gov NCT00521950). Blood samples for metabolite measurement were collected at $T 1$. Leukopenia was defined by leukocyte counts $<3.0 \times 10^{9} /$ I. For comparison, patients without leukopenia who completed the eight weeks on stable dose were selected from the first 272 included patients of the TOPIC trial.

\section{Results}

Thirty-two patients with and 162 patients without leukopenia were analysed. $T 1$ threshold concentrations of $213 \mathrm{pmol} / 8 \times 10^{8}$ red blood cells (RBC) for $6-\mathrm{TGN}$ and $3,525 \mathrm{pmol} / 8 \times 10^{8} \mathrm{RBC}$ for 6-MMPR were defined: patients exceeding these values were at increased leukopenia risk (OR 6.2 (95\% Cl: $2.8-13.8)$ and $5.9(95 \% \mathrm{Cl}: 2.7-13.3)$, respectively). Leukopenia rates were higher in patients treated with 6-mercaptopurine, compared to azathioprine (OR 7.3 (95\% Cl: 3.1-17.0)), and concurrent anti-TNF therapy (OR $5.1(95 \% \mathrm{Cl}$ : 1.6-16.4)). Logistic regression analysis of the threshold concentrations, thiopurine type and concurrent anti-TNF therapy revealed that elevations of both T1 6-TGN and 6-MMPR resulted in the highest risk for leukopenia, followed by exceeding only the T1 6-MMPR or T1 6-TGN threshold concentration.

Conclusion

Early assessment of both 6-TGN and 6-MMPR metabolite concentrations one week after thiopurine initiation identifies patients at increased risk of thiopurine-induced leukopenia. 


\section{Introduction}

For both the immunomodulating thiopurine drugs azathioprine (AZA) and 6-mercaptopurine (6MP) therapeutic efficacy has been demonstrated with respect to induction and maintenance of remission in patients with inflammatory bowel diseases (IBD; Crohn's disease (CD) and ulcerative colitis (UC)). ${ }^{1-3}$ Unfortunately, up to $25 \%$ of IBD patients discontinue thiopurine therapy during the first months of treatment due to adverse events. ${ }^{4-6}$ The most important and potentially life-threatening adverse reaction of AZA/6MP treatment is myelosuppression. A review of thiopurine-induced myelotoxicity in patients with IBD, including data from 66 trials (8,302 patients), reported on a $7 \%$ cumulative incidence of $A Z A / 6 M P$-induced myelotoxicity, which predominantly occurs within the first weeks or months of treatment. ${ }^{7}$

AZA and 6MP undergo extensive intracellular metabolic transformations to active metabolites, of which 6-thioguanine nucleotides (6-TGN) and 6-methylmercaptopurine ribonucleotides (6-MMPR) are considered to be the most important (Figure 6.1). Interindividual differences in therapeutic response and toxicity to thiopurines can be explained by variation in the formation of these metabolites, which is partly related to genetic variants in the genes encoding for crucial enzymes in the thiopurine metabolism. $^{8}$

One of the key enzymes in the delicate balance between 6-MMPR and 6-TGN metabolite formation is thiopurine S-methyltransferase (TPMT). ${ }^{8}$ In Caucasians, the distribution of TPMT activity is trimodal which can be related to genetic variants in the TPMT gene: $11 \%$ of the population is a heterozygous carrier of an inactive TPMT allele and $0.3 \%$ is homozygous for a TPMT variant, resulting in an intermediate and low/absent TPMT activity, respectively. Humans carrying two active alleles, approximately 89\%, show normal TPMT enzyme activity. Decreased TPMT enzyme activity shifts thiopurine metabolism towards increased formation of active cytotoxic 6-TGN metabolites. ${ }^{9}$ Prior-to-treat TPMT genotype or phenotype assessment may help to identify patients at risk of developing severe myelotoxicity. ${ }^{10-13}$ However, the predictive value of these assessments is considered to be limited, as in only a quarter of the patients myelotoxicity could be related to TPMT deficiency. ${ }^{14,15}$ Recently, we demonstrated in a large randomised controlled trial that prior-to-treat TPMT genotypebased dosing results in a significant reduction of the occurrence of leukopenia in patients carrying a genetic TPMT variant. However, genotyping of the three most common genetic variants in TPMT $\left({ }^{*} 2, * 3 A\right.$ and $\left.{ }^{*} 3 C\right)$ did not explain all cases of leukopenia. $^{16}$

Steady-state 6-TGN and 6-MMPR metabolite concentrations are generally reached after approximately four weeks of therapy. ${ }^{17,18}$ The therapeutic range has been defined as a steady-state 6 -TGN concentration between 235 and $490 \mathrm{pmol} / 8 \times 10^{8}$ red blood cells (RBC). ${ }^{19,20}$ The risk of leukopenia increases for 6-TGN steady-state concentrations higher than $490 \mathrm{pmol} / 8 \times 10^{8} \mathrm{RBC}$, whereas 6-MMPR concentrations higher than $5,700 \mathrm{pmol} / 8 \times 10^{8} \mathrm{RBC}$ are associated with hepatotoxicity. ${ }^{17,19,21}$ In addition, extremely 
elevated 6-MMPR metabolites have also been associated with severe myelosuppression. $^{22-24}$

Since myelosuppression is linked to high steady-state 6-TGN and 6-MMPR metabolite concentrations, we hypothesize that elevations of these metabolites may already be detectable before steady-state levels are reached. ${ }^{17,19,25,26}$ The aim of present study was to evaluate the predictive value of 6-TGN and 6-MMPR metabolite concentrations, assessed one week after initiating thiopurine therapy for the development of leukopenia during the first 8 weeks of thiopurine treatment.

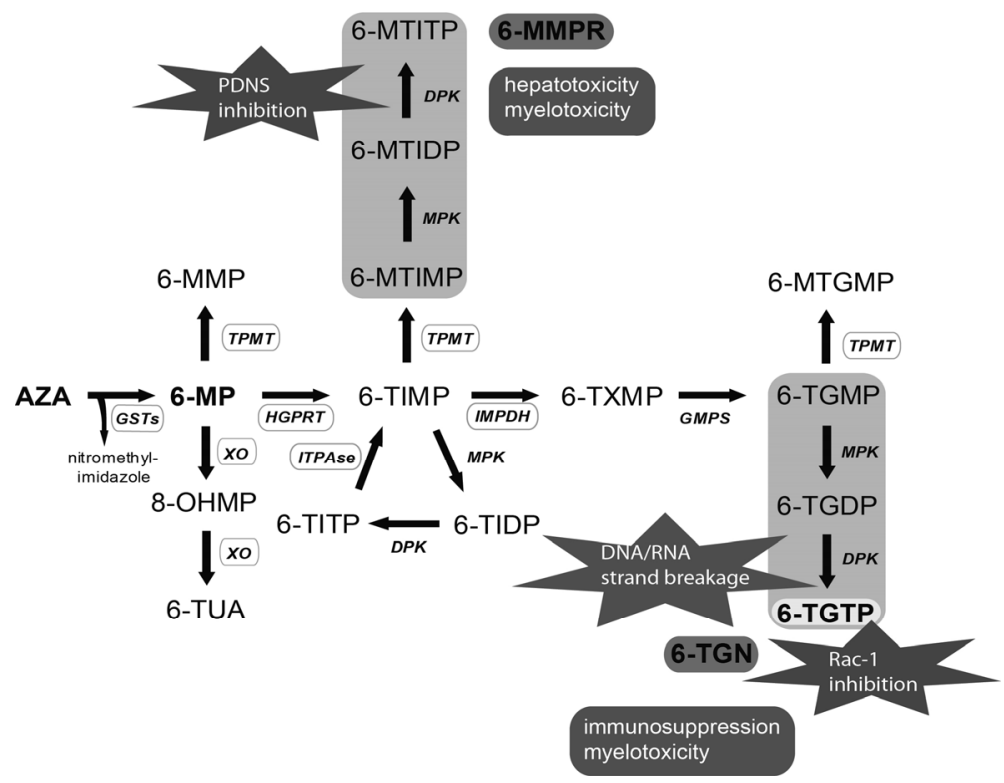

Figure 6.1 Proposed thiopurine metabolism.

AZA, azathioprine; 6MP, 6-mercaptopurine; 6-MMP, 6-methylmercaptopurine; 8-OHMP, 8-hydroxy-6-mercaptopurine; 6-TUA, 6-thiouric acid; 6-MTIMP, 6-methylthioinosine monophosphate; 6-MTIDP, 6-methylthioinosine diphosphate; 6-MTITP, 6-methylthioinosine triphosphate; 6-TIMP, 6-thioinosine monophosphate; 6-TIDP, 6-thioinosine diphosphate; 6-TITP, 6-thioinosine triphosphate; 6-TXMP, 6-thioxanthosine monophosphate; 6-TGMP, 6-thioguanine monophosphate; 6-TGDP, 6-thioguanine diphosphate; 6-TGTP, 6-thioguanine triphosphate; 6-MTGMP, 6-methylthioguanine monophosphate; 6-TG, 6-thioguanine; 6-MTG, 6-methylthioguanine; XO, xanthine oxidase; GST, glutathione-S-transferase; TPMT, thiopurine S-methyl transferase; HGPRT, hypoxanthine phosphoribosyl transferase; IMPDH, inosine monophosphate dehydrogenase; GMPS, guanosine monophosphate synthetase; MPK, monophosphate kinase; DPK, diphosphate kinase; ITPase, inosine triphosphate pyrophosphatase. PDNS, purine de novo synthesis; DNA, deoxyribonucleic acid; RNA, ribonucleic acid. 6-MTIMP, 6-MTIDP and 6-MTITP together form the 6-methylmercaptopurine ribonucleotides (6-MMPR). 6-TGMP, 6-TGDP and 6-TGTP together form the 6-thioguaninenucleotides (6-TGN). Enzymes encoded by genes that are subject to known genetic polymorphisms are circled in grey. (Adapted from Derijks et al. ${ }^{8}$ ). 


\section{Materials and methods}

\section{Study design and outcome definition}

The study was conducted in a subset of the patients from the TOPIC (Thiopurine response Optimization by Pharmacogenetics testing in Inflammatory bowel disease Clinics) trial, a randomised controlled multi-centre trial, including 769 IBD patients, that evaluated the effectiveness of pre-treatment TPMT genotyping. The TOPIC trial was approved by the Medical Ethics Committees of the participating centres. Written informed consent was obtained from all participating patients. The study is registered at clinicaltrials.gov (NCT00521950).

For a detailed description of the study design and patient selection of the TOPIC trial, we refer to Coenen et al.. ${ }^{16}$

Briefly, patients were assessed for eligibility by their gastroenterologist. The inclusion criteria were: age $>18$ years, diagnosis of IBD and indication for AZA or 6MP treatment. Exclusion criteria were: previous use of AZA or 6MP, treatment with allopurinol, known TPMT enzyme activity or genotype, leukocyte count $<3.0 \times 10^{9} /$ l, liver test abnormalities (alanine transaminase, aspartate transaminase, alkaline phosphatase, and/or gamma glutamate transpeptidase $\geq$ twice the upper limit of normal values), impaired renal function (serum creatinine level $\geq$ twice the upper limit of normal) and current pregnancy.

Patients meeting the inclusion criteria were randomly assigned to either standard thiopurine treatment (AZA $2-2.5 \mathrm{mg} / \mathrm{kg} /$ day or $6 \mathrm{MP} 1-1.5 \mathrm{mg} / \mathrm{kg} / \mathrm{day}$ ) or a dose regimen based on the pre-treatment revealed TPMT genotype. Patients of the intervention group carrying a genetic variant were treated with $50 \%$ (heterozygous TPMT) or $0-10 \%$ (homozygous TPMT) of the standard thiopurine dose, according to the guidelines of the Dutch Pharmacogenetics Working Group. ${ }^{27}$

All patients were clinically followed for 20 weeks after treatment initiation. Safety was evaluated by measurement of haematological and biochemical parameters one week before initiation and at least at weeks 1, 2, 4, 6, 8 and 20 after initiation of thiopurine therapy.

For the present study, blood samples for 6-TGN and 6-MMPR assessment were collected one week (i.e. $\sim 7 \pm 1$ day) after thiopurine initiation (T1).

Leukopenia was defined by leukocyte counts $<3.0 \times 10^{9} /$ I. Thiopurine dose adjustments were defined as thiopurine dose escalation or reduction. Any decision regarding dose adjustments or discontinuation of thiopurine therapy was made by the responsible physician and was recorded.

For comparison, patients of the first consecutive 272 patients of the TOPIC trial who completed a follow-up period of 8 weeks without leukopenia on stable thiopurine dose were eligible. Patients with thiopurine dose adjustments or thiopurine discontinuation 
during the first 8 weeks, which were not related to leukopenia (leukocyte count $\left.<3.0 \times 10^{9} / \mathrm{I}\right)$, were excluded from analysis.

Thiopurine metabolite concentrations were assessed in the first 272 consecutive included IBD patients and in the remaining patients included in the TOPIC trial who developed leukopenia in the first 8 weeks after treatment initiation.

In this study the association between 6-TGN and 6-MMPR metabolite concentrations and leukopenia was evaluated, therefore dose reduction based on prior-to-treat TPMT genotyping in the course of the TOPIC trial did not interfere with the primary outcome. The design of the study and flow chart for the patient selection are depicted in Figure 6.2 .

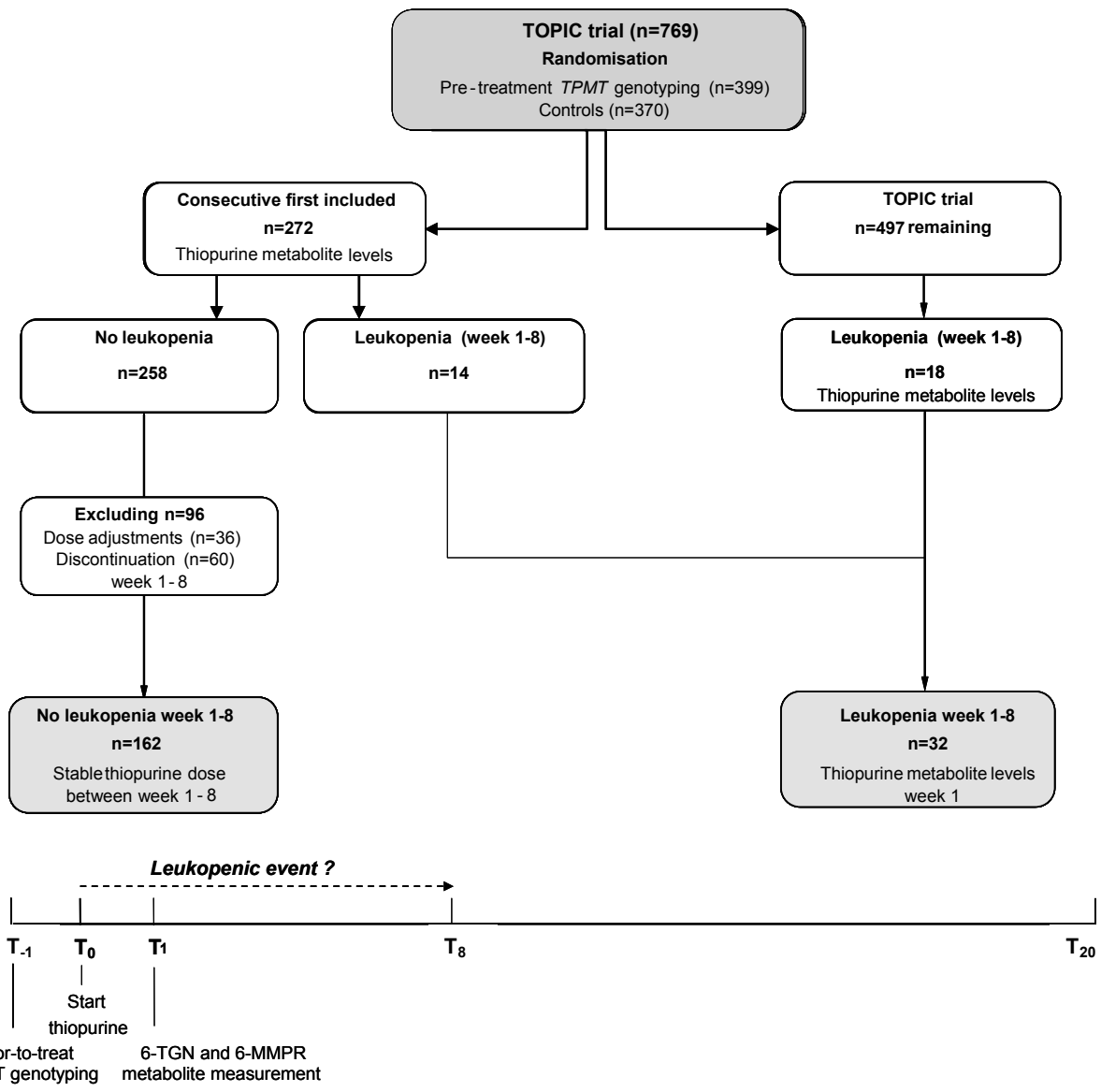

Figure 6.2 Study design and time-line within the TOPIC-trial. 


\section{Measurement of 6-TGN and 6-MMPR metabolite concentrations}

The 6-TGN and 6-MMPR metabolite concentrations were measured after the follow-up period of the TOPIC trial. The thiopurine metabolite concentrations were determined by the modified high performance liquid chromatography (HPLC) method of Lennard and Singleton, as published previously. ${ }^{17}$ The lower-limits of quantification for 6-TGN and 6-MMPR metabolite levels were $40 \mathrm{pmol} / 8 \times 10^{8} \mathrm{RBC}$ and $300 \mathrm{pmol} / 8 \times 10^{8} \mathrm{RBC}$, respectively. To secure metabolite stability, blood samples were immediately stored in the refrigerator $\left(2-8^{\circ} \mathrm{C}\right)$ and subsequently sent to the laboratory of the Department of Clinical Pharmacy and Toxicology of the Orbis Medical Centre (Sittard-Geleen, The Netherlands), where the erythrocytes were washed, counted and stored at $-20^{\circ} \mathrm{C}$ until required.

\section{Data analysis}

Descriptive data analysis was performed by percentages for categorical variables, and by median values and ranges for continuous characteristics. Box-and-whisker plots served as graphical aid. Differences in patient characteristics were evaluated using Pearson's chi-square or Fisher's exact tests; for differences in metabolite concentrations the Mann-Whitney $U$ test or Kolmogorov-Smirnov $Z$ test were applied, when appropriate. P-values of less than 0.05 (two-sided) were considered to be statistically significant. SPSS for windows version 20.0 was used for all data analysis.

To study the discriminative power of 6-TGN and 6-MMPR concentrations regarding leukopenia Receiver Operating Characteristic (ROC) curves were generated, which plot sensitivity rates and false positive rates (1-specificity) for the occurrence of leukopenia. As a summary measure, the area under the ROC curves (AUC) and $95 \%$ confidence interval $(95 \% \mathrm{Cl})$ were calculated.

The predictive power of the metabolites for the development of leukopenia was studied by logistic regression modelling taking covariates into account such as age, gender, disease type (CD/UC), and medication (thiopurine type, thiopurine dose and concurrent use of known potential myelotoxic drugs or drugs interfering with thiopurine metabolism, i.e. mesalazine (5-ASA), anti-TNF therapy (i.e. infliximab (IFX), adalimumab (ADA)).

The results are expressed as odds ratios (OR) with $95 \% \mathrm{Cl}$.

\section{Results}

\section{Baseline cohort analysis}

Fourteen (5\%) of the first 272 consecutive IBD patients included in the TOPIC trial developed leukopenia during the first 8 weeks. Ninety-six (37\%) of the 258 patients without a leukopenic event were excluded from analysis due to thiopurine dose 
adjustments $(n=36,14 \%)$ or treatment discontinuation $(n=60,23 \%)$ during the first 8 weeks, leaving 162 patients without leukopenia for evaluation. Eighteen patients of the remaining 497 patients (4\%) included in the TOPIC trial developed leukopenia during the first 8 weeks of thiopurine therapy and were additionally included, resulting in a total number of 32 patients with leukopenia (Figure 6.2).

Baseline characteristics of the leukopenic and non-leukopenic patient groups are given in Table 6.1. Overall, gender, age, IBD type and thiopurine dose were similar in both patient groups.

Table 6.1 Baseline characteristics of the study population.

\begin{tabular}{|c|c|c|c|c|}
\hline & All patients & Leukopenia & No leukopenia & $\mathrm{p}$-value ${ }^{1}$ \\
\hline Patients (n) & 194 & 32 & 162 & \\
\hline Age in years (median, range) & $38(19-81)$ & $43(19-76)$ & $40(19-81)$ & 0.160 \\
\hline Gender (male / female) & $86 / 108$ & $14 / 18$ & 72 / 90 & 0.942 \\
\hline Bodyweight in kg (median, range) & $70(40-160)$ & $65(50-160)$ & $71(40-100)$ & 0.411 \\
\hline \multicolumn{5}{|l|}{ IBD } \\
\hline Crohn's disease $(n)^{*}$ & 112 & $16(50 \%)$ & 96 (59\%) & \\
\hline Ulcerative colitis $(\mathrm{n})^{*}$ & 81 & $16(50 \%)$ & $65(40 \%)$ & $0.313^{*}$ \\
\hline Indeterminate colitis (n) & 1 & 0 & 1 & \\
\hline $\begin{array}{l}\text { Disease duration in years } \\
\text { (median; range) (missing } n=1 / 1)\end{array}$ & $2.1(0-50)$ & $4.1(0-50)$ & $1.7(0-42)$ & 0.171 \\
\hline \multicolumn{5}{|l|}{ Medication } \\
\hline Azathioprine / 6-mercaptopurine (n) & $129 / 65$ & $9 / 23$ & $120 / 42$ & $<0.000001$ \\
\hline Azathioprine $(\mathrm{n})$ & $129(66 \%)$ & $9(28 \%)$ & $120(74 \%)$ & \\
\hline median AZA dose in mg (range) & $150(50-250)$ & $150(50-225)$ & $150(50-250)$ & 0.723 \\
\hline median AZA dose in $\mathrm{mg} / \mathrm{kg}$ (range) & $2.2(0.8-3.1)$ & $2.2(1.3-2.3)$ & $2.2(0.8-3.1)$ & 0.781 \\
\hline 6-mercaptopurine $(n)$ & $65(34 \%)$ & $23(72 \%)$ & $42(26 \%)$ & \\
\hline median 6-MP dose in mg (range) & $75(25-150)$ & $75(25-150)$ & $75(50-125)$ & 0.782 \\
\hline median 6-MP dose in $\mathrm{mg} / \mathrm{kg}$ (range) & $1.2(0.4-1.5)$ & $1.2(0.4-1.5)$ & $1.2(0.6-1.4)$ & 0.351 \\
\hline \multicolumn{5}{|l|}{ Concurrent IBD medication (n) } \\
\hline 5-ASA & $110(57 \%)$ & $16(50 \%)$ & $94(58 \%)$ & 0.402 \\
\hline Corticosteroids & $160(82 \%)$ & $25(78 \%)$ & $135(83 \%)$ & 0.479 \\
\hline Anti-TNF- $\alpha$ (i.e. IFX, ADA) & $13(7 \%)$ & $6(19 \%)$ & $7(4 \%)$ & 0.006 \\
\hline
\end{tabular}

${ }^{1} \mathrm{p}$-value $<0.05$ was considered to be statistically significant.

The median time to onset of leukopenia was 4.8 weeks (range 1.0-8.3) after thiopurine initiation.

Leukopenia rates were higher in patients treated with $6 \mathrm{MP}$, when compared to AZA (OR 7.3 (95\% Cl: 3.1-17.0)). Patients concomitantly treated with anti-TNF therapy at baseline, developed significantly more often leukopenia, when compared to patients without (OR 5.1 (95\%Cl: 1.6-16.4)). 
6-TGN and 6-MMPR concentrations at week 1 were higher in patients treated with $6 \mathrm{MP}$, compared to AZA. In addition, 6-MMPR concentrations were higher in women at week 1, compared to men. Concomitant treatment with systemic corticosteroids, 5-ASA and anti-TNF- $\alpha$ treatment did not influence the 6-TGN and 6-MMPR concentrations at week 1 (Table 6.2).

Table 6.2 Thiopurine metabolite concentrations at week 1 for different patient characteristics.

\begin{tabular}{|c|c|c|c|c|}
\hline & & Patient number $(\mathrm{n})$ & T1 6-TGN ${ }^{*}$ & T1 6-MMPR ${ }^{*}$ \\
\hline \multicolumn{2}{|l|}{ All Patients } & 194 & $159(40-641)$ & $2,133(300-13,576)$ \\
\hline \multicolumn{5}{|l|}{ Gender } \\
\hline \multirow{2}{*}{\multicolumn{2}{|c|}{$\begin{array}{l}\text { male } \\
\text { female }\end{array}$}} & 86 & $150(42-528)$ & $1,605(300-13,576)$ \\
\hline & & 108 & $168(40-641)$ & $2,750(300-11,705)$ \\
\hline \multicolumn{2}{|l|}{$p$-value } & & 0.165 & 0.001 \\
\hline \multicolumn{5}{|l|}{ IBD } \\
\hline \multicolumn{2}{|l|}{ Crohn's disease } & 112 & $166(40-641)$ & $2,283(300-13,576)$ \\
\hline \multicolumn{2}{|l|}{ Ulcerative colitis } & 81 & $147(42-607)$ & $1,798(300-13,348)$ \\
\hline \multicolumn{2}{|l|}{ p-value } & & 0.286 & 0.402 \\
\hline \multicolumn{5}{|c|}{$\mathrm{n}=1$ missing (indeterminate colitis) } \\
\hline \multicolumn{5}{|l|}{ Thiopurine type } \\
\hline \multicolumn{2}{|l|}{ AZA } & 129 & $139(40-607)$ & $1,812(300-13,576)$ \\
\hline \multicolumn{2}{|l|}{$6 \mathrm{MP}$} & 65 & $207(76-641)$ & $2,725(300-13,348)$ \\
\hline \multicolumn{2}{|l|}{ p-value } & & $<0.000001$ & 0.009 \\
\hline \multicolumn{5}{|c|}{ Concurrent IBD medication } \\
\hline \multirow[t]{3}{*}{ 5-ASA } & yes & 110 & $164(40-607)$ & $2,006(300-11,705)$ \\
\hline & no & 84 & $156(40-641)$ & $2,185(300-13,576)$ \\
\hline & $p$-value & & 0.259 & 0.398 \\
\hline \multirow[t]{3}{*}{ Corticosteroids } & yes & 170 & $156(40-641)$ & $2,133(300-13,576)$ \\
\hline & no & 35 & $163(40-607)$ & $2,171(300-11,230)$ \\
\hline & p-value & & 0.168 & 0.753 \\
\hline \multirow[t]{3}{*}{ Anti-TNF- $\alpha$ (IFX, ADA) } & yes & 13 & $176(51-459)$ & $3,241(300-13,576)$ \\
\hline & no & 181 & $158(40-641)$ & $2,092(300-13,348)$ \\
\hline & p-value & & 0.484 & 0.258 \\
\hline
\end{tabular}

*6-TGN and 6-MMPR concentrations ( $\mathrm{pmol} / 8 \times 10^{8} \mathrm{RBC}$ ) are presented as median values and ranges.

\section{Thiopurine metabolite concentrations at week 1 in relation to leukopenia}

At week 1, the median 6-TGN concentration in patients who developed leukopenia within the first 8 weeks $(n=32)$ was higher than in patients who did not $(n=162): 240$

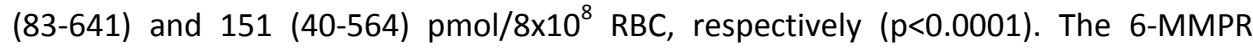
levels were also higher in patients who developed leukopenia $(4,051(300-13,576)$ versus $\left.1,834(300-7,996) \mathrm{pmol} / 8 \times 10^{8} \mathrm{RBC}, \mathrm{p}<0.0001\right)$ (Figure 6.3). The median values of the 6-MMPR/6-TGN ratio were not different between the leukopenic and nonleukopenic patients: 17 (1-117) and 14 (1-64), respectively $(p=0.190)$.

As concomitant anti-TNF therapy was associated with the development of leukopenia, we also performed an analysis excluding 13 patients treated with infliximab or 


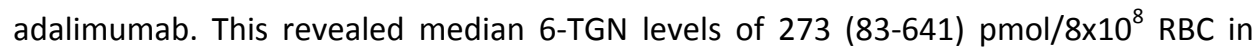
leukopenic patients $(n=26)$ and 151 (40-564) pmol/8x $10^{8}$ RBC in non-leukopenic patients $(n=155) \quad(p<0.0001)$. The median 6-MMPR concentrations were 3,725 $(430-13,348)$ and $1,849(300-7,996) \mathrm{pmol} / 8 \times 10^{8} \mathrm{RBC}$, respectively $(\mathrm{p}=0.001)$.
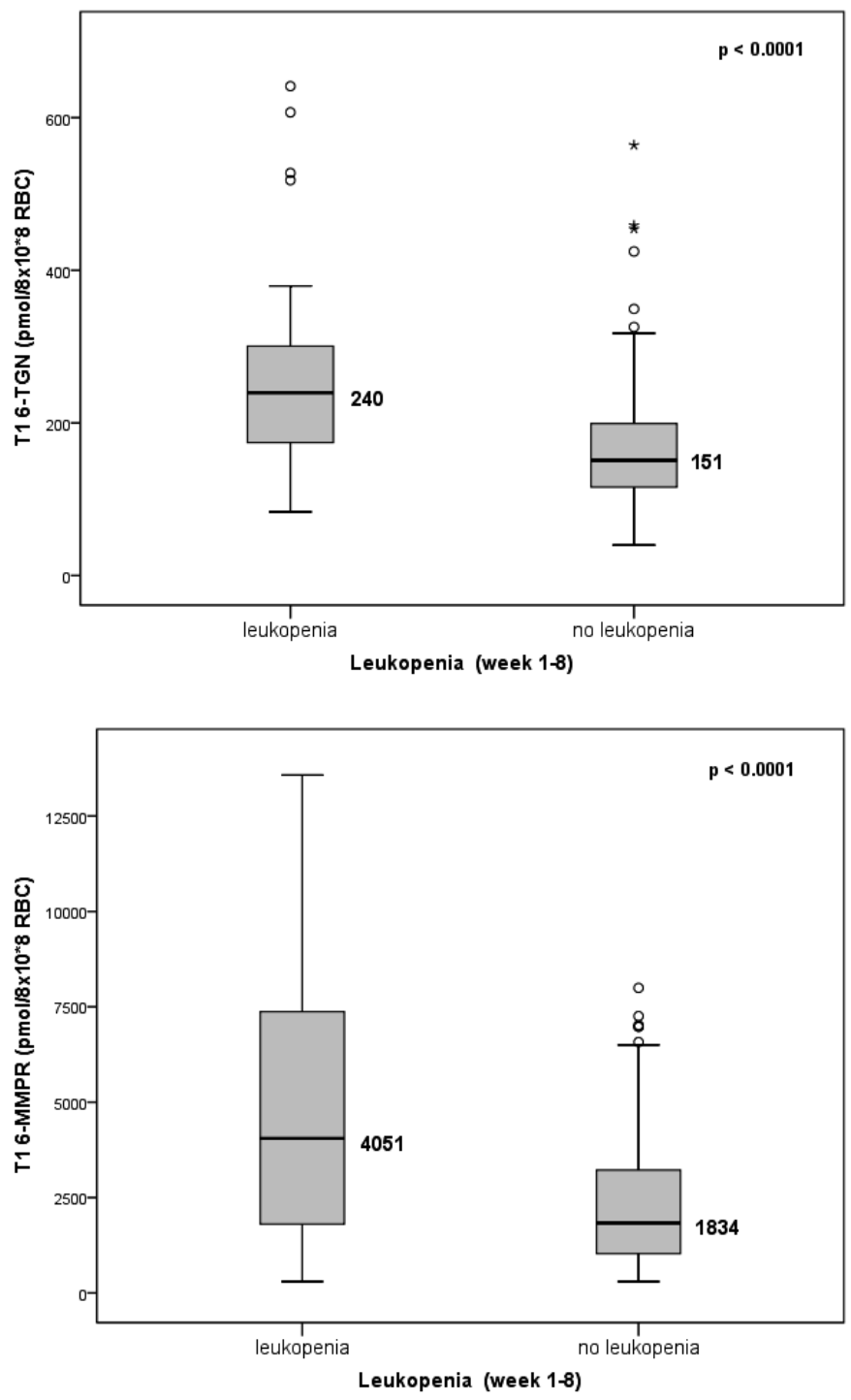

Figure 6.3 Box-and-whisker plots of 6-TGN and 6-MMPR concentrations at week 1 in patients who developed leukopenia $(n=32)$ and patients who did not develop leukopenia $(n=162)$ in week 1-8. 
The area under the ROC curves of the continuous 6-TGN concentration for the occurrence of leukopenia was 0.73 (95\% Cl: $0.63-0.84)$, and 0.72 (95\% Cl: $0.61-0.83)$ for 6-MMPR (Figure 6.4). Excluding patients with concurrent anti-TNF therapy at baseline, resulted in areas under the ROC curves of $0.76(95 \% \mathrm{Cl}: 0.64-0.87)$ for $6-\mathrm{TGN}$ and 0.70 (95\% Cl: 0.58-0.82) for 6-MMPR.

\section{6-TGN and 6-MMPR threshold values}

Based on contingency tables of T1 6-TGN and 6-MMPR concentration quartiles for the development of leukopenia, we defined threshold values for 6-TGN to be $213 \mathrm{pmol} / 8 \times 10^{8} \mathrm{RBC}$ and $3,525 \mathrm{pmol} / 8 \times 10^{8} \mathrm{RBC}$ for $6-\mathrm{MMPR}\left(75^{\text {th }}\right.$ percentiles). The associated specificity and sensitivity rates of $80 \%$ and $59 \%$, respectively, were considered clinical useful. Patients with 6 -TGN levels $>213 \mathrm{pmol} / 8 \times 10^{8} \mathrm{RBC}$ were at significantly increased risk of leukopenia, OR $6.2(95 \% \mathrm{Cl}: 2.8-13.8)$. A similar risk was observed for patients with 6-MMPR levels $>3525 \mathrm{pmol} / 8 \times 10^{8} \mathrm{RBC}$, OR $5.9(95 \% \mathrm{Cl}$ : 2.7-13.3). The discriminative performance of 6-TGN and 6-MMPR dichotomised at threshold, measured by $\mathrm{ROC}$ analysis, revealed an $\mathrm{AUC}=0.70(95 \% \mathrm{Cl}: 0.59-0.81)$ for both.

The predictive power for three cut-off values of 6-TGN and 6-MMPR concentrations at $T 1$, set at specificity rates of 80,90 and $95 \%$, are presented in Table 6.3. Odds ratios serve as overall measure, whereas positive and negative likelihood ratios (true positive versus false positive and false negative versus true negative) facilitate medical practice. The ranges of $O R$, positive and negative likelihood ratios do not reveal preference for a specific threshold value.

Prediction model for leukopenia including thiopurine type and concurrent anti-TNF treatment

A multivariable logistic regression analysis was performed based on the 6-TGN and 6-MMPR threshold parameters, thiopurine type (6MP or AZA) and concomitant antiTNF treatment to predict the development of leukopenia. Patients treated with AZA monotherapy and both 6-TGN and 6-MMPR levels below the defined threshold values were used as the reference group. Patients treated with 6MP monotherapy exceeding either the 6-MMPR or 6-TGN threshold concentration were at increased risk of leukopenia (OR 10.2 (95\% Cl: 2.0-51.6) and OR 11.7 (95\% Cl: 2.5-55.2), respectively). Patients with both elevated 6-TGN and 6-MMPR concentrations were at highly increased risk of developing leukopenia: OR 65.3 (95\% Cl: 11.1-386) and OR $28.0(95 \% \mathrm{Cl}$ : 2.9-271.9) for 6MP and AZA therapy, respectively. The risk of leukopenia was highest among patients concomitantly treated with anti-TNF therapy with elevated 6-TGN and 6-MMPR concentrations: all patients $(n=4)$ treated with 6MP developed leukopenia. The area under the ROC curve for the obtained predicted probabilities based on the four parameters was 0.84 (95\% Cl: 0.76-0.92) (Figure 6.4). 
Table 6.3 Predictive power of three cut-off threshold values of 6-TGN and 6-MMPR concentrations set on specificity rates of $80 \%, 90 \%$ and $95 \%$ for thiopurine-induced leukopenia.

\begin{tabular}{llllll}
\hline Threshold value & Specificity (\%) & Sensitivity (\%) & $\begin{array}{l}\text { Odds ratio } \\
(95 \% \mathrm{Cl})\end{array}$ & $\begin{array}{l}\text { Positive } \\
\text { Likelihood ratio }\end{array}$ & $\begin{array}{l}\text { Negative } \\
\text { Likelihood ratio }\end{array}$ \\
\hline $6-\mathrm{TGN}$ & & & & & \\
213 & 80 & 59 & 6.2 & 3 & 0.5 \\
245 & 90 & 50 & 9.1 & 5 & 0.6 \\
301 & 95 & 25 & 6.4 & 5 & 0.8 \\
$6-\mathrm{MMPR}$ & & & & & \\
3,525 & 80 & 59 & 5.9 & 3 & 0.5 \\
5,220 & 90 & 44 & 7.1 & 4 & 0.6 \\
6,270 & 95 & 38 & 11.6 & 8 & 0.7 \\
\hline
\end{tabular}

* Thiopurine metabolite concentrations are given in $\mathrm{pmol} / 8 \times 10^{8} \mathrm{RBC}$.

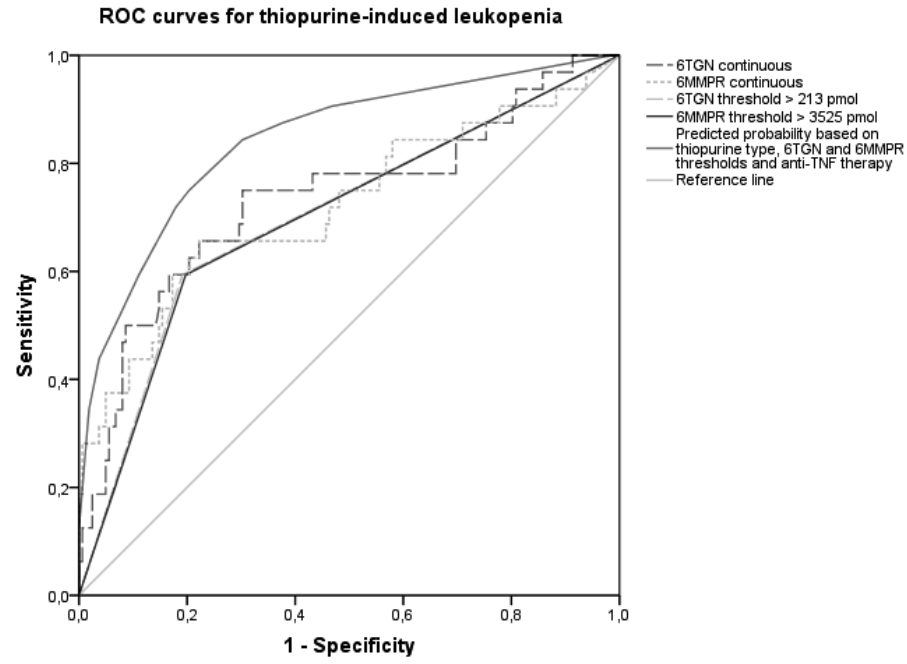

Figure 6.4 Receiver operating characteristic (ROC) curves for 6-TGN and 6-MMPR metabolite concentrations at week 1 with the threshold parameters, thiopurine type and concomitant antiTNF therapy.

\section{Discussion}

In this prospective study we showed for the first time that elevated 6-TGN and 6-MMPR metabolite levels assessed one week after initiation are highly predictive for thiopurineinduced leukopenia during the first 8 weeks of thiopurine therapy in IBD patients.

In previous studies the relation between thiopurine metabolites and leukopenia has been studied during steady-state maintenance therapy, while leukopenia frequently 
occurs in the early stage of therapy, mostly before steady-state concentrations are reached. $^{19,24}$ Therefore, in clinical practice steady-state thiopurine metabolite concentrations are less useful to identify patients at risk to develop leukopenia during the first weeks of thiopurine treatment.

Early identification of patients at increased risk of leukopenia is important as it may reduce morbidity, and to a (much) lesser extent hospitalisation or mortality.

ROC analyses of the 6-TGN and 6-MMPR concentrations predicting leukopenia revealed an area under the ROC curve of 0.73 and 0.72 , respectively, indicating a proper discriminative performance for both thiopurine metabolites. Until now, myelotoxicity has mainly been attributed to elevated 6-TGN concentrations. ${ }^{19,21}$ To our surprise, we observed that the predictive value for the occurrence of leukopenia of the 6-MMPR metabolites at week 1 is similar to the 6-TGN metabolites.

Only a limited number of studies related the development of leukopenia during maintenance therapy to the methylated thiopurine metabolites. Hindorf et al. reported that only 6-methylthioinosine monophosphate (6MTIMP), the first methylated metabolite of the 6-MMPR, was associated with myelotoxicity in IBD patients following a dose escalation schedule for AZA and 6MP. ${ }^{24}$ In addition, two case reports also indicated that extremely elevated 6-MMPR levels with subtherapeutic 6-TGN levels can cause severe myelosuppression. ${ }^{22,23}$

The interpretation of the findings from previous studies on the relation between thiopurine metabolites and myelotoxicity is complex, as often different definitions of leukopenia and myelosuppression (leukopenia, thrombocytopenia and/or neutropenia) were used, and the association was mostly studied during different phases of thiopurine therapy. In addition, different thiopurine metabolites were evaluated and determined by different analytical HPLC methods in relatively small number of patients. ${ }^{17,19,24,28,29}$ These factors make the data hard to compare and to apply these in clinical practice.

The present study results show that both 6-TGN and 6-MMPR are independently correlated with leukopenia and that the cytotoxic effect is presumably enhanced by one another. The cytotoxic effect of the 6-TGN has been ascribed to incorporation into DNA, acting as fraudulent bases resulting in strand breakage. ${ }^{30,31}$ In addition, the 6-MMPR, in particular the cytotoxic metabolite 6MTIMP, have been shown to inhibit the purine de novo synthesis (PDNS) leading to inhibition of DNA formation, decreased cell proliferation and cytotoxic effects. ${ }^{31,32}$ It has even been suggested that AZA and 6MP exert the cytotoxic effect mainly through PDNS inhibition, independently of 6-TGN formation. ${ }^{31}$ The latter may explain, at least for a part, the fact that thiopurine-induced leukopenia is not always related to elevated 6-TGN concentrations due to low TPMT enzyme activity.

Myelosuppression is often multi-factorial or caused by other factors, such as viral infections (e.g. Parvovirus B19, Varicella zoster), medication causing myelosuppression by itself (e.g. captopril, metronidazole or trimethotrim-sulfamethoxazole) or medication interfering with thiopurine metabolism (i.e. allopurinol, mesalazine). ${ }^{14,33}$ 
Viral infections have not been evaluated in our study, due to lack of data. We did evaluate concomitant IBD medication (mesalazine and anti-TNF therapy) for the occurrence of leukopenia. Concurrent treatment of 5-ASA has been demonstrated to result in elevated 6-TGN concentrations, which may lead to an increased risk for development of myelotoxicity. ${ }^{34-36}$ In the present study, we did not confirm this for the metabolite concentrations at week 1 and the occurrence of leukopenia during the first 8 weeks of treatment. However, anti-TNF agents appeared to be an independent risk factor for leukopenia in patients concomitantly treated with thiopurines, while thiopurine metabolite concentrations at week 1 were not affected. Consequently, this finding indicates that the myelotoxic properties of thiopurines may be potentiated by additional myelotoxic effects of anti-TNF agents, as we have previously described for concurrent adalimumab therapy in a small CD patient group showing mainly lowtherapeutic 6-TGN concentrations. ${ }^{37}$ This finding is clinically relevant as combined antiTNF and thiopurine therapy seems to have beneficial therapeutic effects, at least in the treatment of moderate-to-severe active inflammatory bowel disease. ${ }^{38-40}$

Some remarks should be made concerning our study design and patient selection. First, leukopenia was defined by a leukocyte count $<3.0 \times 10^{9} / \mathrm{l}$ in order to study the relation between thiopurine metabolites and moderate-to-severe leukopenia. We were unable to evaluate the 6-TGN and 6-MMPR metabolite concentrations during any leukopenic event. Second, patients of whom thiopurine dose was adjusted by the clinician due to any adverse event, but not related to leukopenia, were excluded from analysis. Consequently, patients whose dose was reduced due to a mild leukopenia (leukocyte count 3.0-4.0 $\times 10^{9} / \mathrm{l}$ ) were not evaluated, possibly explaining the moderate sensitivity (both 59\%) of the defined predictive 6-TGN and 6-MMPR threshold values for developing leukopenia. The moderate sensitivity rates may also be explained by the fact that the metabolite concentrations are assessed in erythrocytes, as a surrogate marker for the actual target cells, the leukocytes. Furthermore, patients who developed leukopenia after the follow-up period of 8 weeks of treatment were not evaluated. Third, in the present study, patients treated with 6MP were at higher risk of leukopenia, compared to patients treated with AZA. Although AZA and 6MP in all patients were equivalently dosed (AZA 2-2.5 mg/kg/day and 6-MP 1-1.5 mg/kg/day), this observation may be partly explained by the fact that the concentrations of 6-TGN and 6-MMPR were both higher in patients treated with 6MP.

The formation of 6-TGN and 6-MMPR metabolites is often inversely related within each individual, so that the predictive value of one may be affected by the other. In addition, patients treated with 6MP and concurrent anti-TNF therapy were more likely to develop leukopenia. Multivariable regression analysis of these determinants revealed that patients with both 6-TGN and 6-MMPR above the predictive threshold concentrations have a highly increased risk of leukopenia, followed by patients with either 6-TGN or 6-MMPR levels above the defined thresholds at week 1. Patients on concurrent anti-TNF therapy with elevated T1 6-TGN and 6-MMPR levels all developed leukopenia. Consequently, based on different combinations of the aforementioned 
parameters, patients can be classified using this accurate predictive algorithm $(A \cup C=0.84)$ to assess the degree of risk for the development of thiopurine-induced leukopenia.

Thiopurine metabolism is complex and unpredictable due to the involvement of various metabolic enzymes, which results in a great interindividual variation of pharmacological active thiopurine metabolite formation. ${ }^{8}$ Assessment of thiopurine metabolites is an attractive way to reveal a patients 'ultimate phenotype', displayed by the proposed most important metabolites, 6-TGN and 6-MMPR.

Early identification of patients at risk for leukopenia is important. Therefore, in addition to pre-treatment TPMT genotype testing, T1 thiopurine metabolite assessment may be helpful to identify patients at risk to develop leukopenia due to high cytotoxic 6-TGN and 6-MMPR concentrations resulting from other causes than a TPMT deficiency, such as overdosing or a skewed thiopurine metabolism towards 6-MMPR formation. In these patients intensive safety monitoring and adequate thiopurine dose reduction is advocated. Of course, in future research the proposed algorithm should be validated in a prospective randomised-controlled intervention study, evaluating whether optimising strategies based on the T1 metabolite profile will prevent the occurrence of leukopenia.

In conclusion, both 6-TGN and 6-MMPR metabolites predict the occurrence of leukopenia during the first 8 weeks of therapy. Assessment of 6-TGN and 6-MMPR metabolite concentrations one week after thiopurine initiation helps to identify patients at risk of developing thiopurine-induced leukopenia. 


\section{References}

1. Prefontaine E, Macdonald JK, Sutherland LR. Azathioprine or 6-mercaptopurine for induction of remission in Crohn's disease. Cochrane Database Syst Rev 2009(4):CD000545.

2. Prefontaine E, Sutherland LR, Macdonald JK, Cepoiu M. Azathioprine or 6-mercaptopurine for maintenance of remission in Crohn's disease. Cochrane Database Syst Rev 2009(1):CD000067.

3. Timmer A, McDonald JW, Macdonald JK. Azathioprine and 6-mercaptopurine for maintenance of remission in ulcerative colitis. Cochrane Database Syst Rev 2007(1):CD000478.

4. Schwab M, Schäffeler E, Marx C, Fischer C, Lang T, Behrens C, Gregor M, Eichelbaum M, Zanger UM, Kaskas BA. Azathioprine therapy and adverse drug reactions in patients with inflammatory bowel disease: impact of thiopurine S-methyltransferase polymorphism. Pharmacogenetics 2002;12:429-36.

5. Jharap B, Seinen ML, de Boer NK, van Ginkel JR, Linskens RK, Kneppelhout JC, Mulder CJ, van Bodegraven AA. Thiopurine therapy in inflammatory bowel disease patients: analyses of two 8-year intercept cohorts. Inflamm Bowel Dis 2010;16:1541-9.

6. Chaparro M, Ordás I, Cabré E, Garcia-Sanchez V, Bastida G, Peñalva M, Gomollón F, García-Planella E, Merino O, Gutiérrez A, Esteve M, Márquez L, Garcia-Sepulcre M, Hinojosa J, Vera I, Muñoz F, Mendoza JL, Cabriada JL, Montoro MA, Barreiro-de Acosta M, Ceña G, Saro C, Aldeguer X, Barrio J, Maté J, Gisbert JP. Safety of thiopurine therapy in inflammatory bowel disease: long-term follow-up study of 3931 patients. Inflamm Bowel Dis 2013;19:1404-10.

7. Gisbert JP, Gomollon F. Thiopurine-induced myelotoxicity in patients with inflammatory bowel disease: a review. Am J Gastroenterol 2008;103:1783-800.

8. Derijks LJ, Wong DR. Pharmacogenetics of thiopurines in inflammatory bowel disease. Curr Pharm Des 2010;16:145-54.

9. Weinshilboum RM, Sladek SL. Mercaptopurine pharmacogenetics: monogenic inheritance of erythrocyte thiopurine methyltransferase activity. Am J Hum Genet 1980;32:651-62.

10. Derijks LJ, Gilissen LP, Hooymans PM, Hommes DW. Review article: thiopurines in inflammatory bowel disease. Aliment Pharmacol Ther 2006;24:715-29.

11. Higgs JE, Payne K, Roberts C, Newman WG. Are patients with intermediate TPMT activity at increased risk of myelosuppression when taking thiopurine medications? Pharmacogenomics 2010;11:177-88.

12. Ujiie S, Sasaki T, Mizugaki M, Ishikawa M, Hiratsuka M. Functional characterization of 23 allelic variants of thiopurine S-methyltransferase gene (TPMT*2 - *24). Pharmacogenet Genomics 2008;18:887-93.

13. Hindorf $U$, Appell ML. Genotyping should be considered the primary choice for pre-treatment evaluation of thiopurine methyltransferase function. J Crohns Colitis 2012;6:655-9.

14. Colombel JF, Ferrari N, Debuysere H, Marteau P, Gendre JP, Bonaz B, Soulé JC, Modigliani R, Touze Y, Catala P, Libersa C, Broly F. Genotypic analysis of thiopurine S-methyltransferase in patients with Crohn's disease and severe myelosuppression during azathioprine therapy. Gastroenterology 2000;118:1025-30.

15. Dewit O, Moreels T, Baert F, Peeters H, Reenaers C, de Vos M, Van Hootegem P, Muls V, Veereman G, Mana F, Van Outryve M, Holvoet J, Naegels S, Piessevaux H, Horsmans Y, Gala JL; Belgian Inflammatory Bowel Disease Research Group (BIRD). Limitations of extensive TPMT genotyping in the management of azathioprine-induced myelosuppression in IBD patients. Clin Biochem 2011;44:1062-6.

16. Coenen MJ, de Jong DJ, van Marrewijk C J, Derijks LJJ, Vermeulen SH, Wong DR, Klungel OH, Verbeek ALM, Hooymans PM, Peters WHM, te Morsche RHM, Newman WG, Scheffer H, Guchelaar H-J, Franke B. Screening of the TPMT gene followed by personalized dosing of azathioprine and mercaptopurine treatment reduces the risk of leucopenia in patients with inflammatory bowel disease: a randomized trial. Submitted for publication 2014.

17. Derijks LJ, Gilissen LP, Engels LG, Bos LP, Bus PJ, Lohman JJ, Curvers WL, Van Deventer SJ, Hommes DW, Hooymans PM. Pharmacokinetics of 6-mercaptopurine in patients with inflammatory bowel disease: implications for therapy. Ther Drug Monit 2004;26:311-8.

18. Lennard L, Singleton HJ. High-performance liquid chromatographic assay of the methyl and nucleotide metabolites of 6-mercaptopurine: quantitation of red blood cell 6-thioguanine nucleotide, 6-thioinosinic acid and 6-methylmercaptopurine metabolites in a single sample. J Chromatogr 1992; 583:83-90. 
19. Dubinsky MC, Lamothe S, Yang HY, Targan SR, Sinnett D, Théorêt Y, Seidman EG. Pharmacogenomics and metabolite measurement for 6-mercaptopurine therapy in inflammatory bowel disease. Gastroenterology 2000;118:705-13.

20. Cuffari C, Theoret Y, Latour S, Seidman G. 6-Mercaptopurine metabolism in Crohn's disease: correlation with efficacy and toxicity. Gut 1996;39:401-6.

21. Seidman EG. Clinical use and practical application of TPMT enzyme and 6-mercaptopurine metabolite monitoring in IBD. Rev Gastroenterol Disord 2003;3 Suppl 1:S30-8.

22. Gilissen LP, Derijks LJ, Verhoeven HM, Bierau J, Hooymans PM, Hommes DW, Engels LG. Pancytopenia due to high 6-methylmercaptopurine levels in a 6-mercaptopurine treated patient with Crohn's disease. Dig Liver Dis 2007;39:182-6.

23. Seinen $M L$, van Bodegraven $A A$, van Kuilenburg $A B$, de Boer NK. High TPMT activity as a risk factor for severe myelosuppression during thiopurine therapy. Neth J Med 2013;71:222.

24. Hindorf $U$, Lindqvist $M$, Peterson C, Söderkvist $P$, Ström M, Hjortswang $H$, Pousette A, Almer S. Pharmacogenetics during standardised initiation of thiopurine treatment in inflammatory bowel disease. Gut 2006;55:1423-31.

25. Lennard L, Lilleyman JS, Van Loon J, Weinshilboum RM. Genetic variation in response to 6-mercaptopurine for childhood acute lymphoblastic leukaemia. Lancet 1990;336:225-9.

26. Cuffari C, Seidman EG, Latour S, Theoret Y. Quantitation of 6-thioguanine in peripheral blood leukocyte DNA in Crohn's disease patients on maintenance 6-mercaptopurine therapy. Can J Physiol Pharmacol 1996;74:580-5.

27. Swen JJ, Nijenhuis M, de Boer A, Grandia L, Maitland-van der Zee AH, Mulder H, Rongen GA, van Schaik $\mathrm{RH}$, Schalekamp T, Touw DJ, van der Weide J, Wilffert B, Deneer VH, Guchelaar HJ. Pharmacogenetics: from bench to byte--an update of guidelines. Clin Pharmacol Ther 2011;89:662-73.

28. Pettersson B, Almer S, Albertioni F, Soderhall S, Peterson C. Differences between children and adults in thiopurine methyltransferase activity and metabolite formation during thiopurine therapy: possible role of concomitant methotrexate. Ther Drug Monit 2002;24:351-8.

29. Armstrong VW, Shipkova M, von Ahsen N, Oellerich M. Analytic aspects of monitoring therapy with thiopurine medications. Ther Drug Monit 2004;26:220-6.

30. Lennard L. The clinical pharmacology of 6-mercaptopurine. Eur J Clin Pharmacol 1992;43:329-39.

31. Coulthard SA, Hogarth LA, Little M, Matheson EC, Redfern CP, Minto L, Hall AG. The effect of thiopurine methyltransferase expression on sensitivity to thiopurine drugs. Mol Pharmacol 2002;62:102-9.

32. Dervieux T, Blanco JG, Krynetski EY, Vanin EF, Roussel MF, Relling MV. Differing contribution of thiopurine methyltransferase to mercaptopurine versus thioguanine effects in human leukemic cells. Cancer Res 2001;61:5810-6.

33. van Asseldonk DP, Kanis BM, de Boer NK, van Bodegraven AA. Leukopenia due to parvovirus B19 in a Crohn's disease patient using azathioprine. Digestion 2009;79:211-4.

34. Lowry PW, Franklin CL, Weaver AL, Szumlanski CL, Mays DC, Loftus EV, Tremaine WJ, Lipsky JJ, Weinshilboum RM, Sandborn WJ. Leucopenia resulting from a drug interaction between azathioprine or 6-mercaptopurine and mesalamine, sulphasalazine, or balsalazide. Gut 2001;49:656-64.

35. de Boer NK, Wong DR, Jharap B, de Graaf P, Hooymans PM, Mulder CJ, Rijmen F, Engels LG, van Bodegraven AA. Dose-dependent influence of 5-aminosalicylates on thiopurine metabolism. Am J Gastroenterol 2007;102:2747-53.

36. Gao X, Zhang FB, Ding L, Liu H, Wang XD, Chen BL, Bi HC, Xiao YL, Zhao LZ, Chen MH, Huang M, Hu PJ. The potential influence of 5-aminosalicylic acid on the induction of myelotoxicity during thiopurine therapy in inflammatory bowel disease patients. Eur J Gastroenterol Hepatol 2012;24:958-64.

37. Wong DR, Pierik M, Seinen ML, van Bodegraven AA, Gilissen LP, Bus P, Bakker JA, Masclee AA, Neef C, Engels LG, Hooymans PM. The pharmacokinetic effect of adalimumab on thiopurine metabolism in Crohn's disease patients. J Crohns Colitis 2014;8:120-8.

38. Colombel JF, Sandborn WJ, Reinisch W, Mantzaris GJ, Kornbluth A, Rachmilewitz D, Lichtiger S, D'Haens G, Diamond RH, Broussard DL, Tang KL, van der Woude CJ, Rutgeerts P; SONIC Study Group. Infliximab, azathioprine, or combination therapy for Crohn's disease. N Engl J Med 2010;362:1383-95.

39. Reenaers C, Louis E, Belaiche J, Seidel L, Keshav S, Travis S. Does co-treatment with immunosuppressors improve outcome in patients with Crohn's disease treated with adalimumab? Aliment Pharmacol Ther 2012;36:1040-8. 
40. Panaccione R, Ghosh S, Middleton S, Márquez JR, Scott BB, Flint L, van Hoogstraten HJ, Chen AC, Zheng $H$, Danese S, Rutgeerts $P$. Combination therapy with infliximab and azathioprine is superior to monotherapy with either agent in ulcerative colitis. Gastroenterology 2014;146:392-400 e3. 


\section{Chapter 7}

Early elevated 6-methylmercaptopurine levels predict thiopurine-induced hepatotoxicity in inflammatory bowel disease

DR Wong, MJH Coenen, LJ Derijks, SH Vermeulen, CJ van Marrewijk, OH Klungel, H Scheffer, B Franke, HJ Guchelaar, DJ de Jong, LGJB Engels, ALM Verbeek, PM Hooymans

\section{Submitted}




\section{Abstract}

\section{Background \& Aims}

Hepatotoxicity, gastrointestinal complaints and general malaise are the most common adverse reactions of azathioprine and 6-mercaptopurine in inflammatory bowel disease (IBD) patients, related to high 6-methylmercaptopurine ribonucleotide (6-MMPR) metabolite concentrations. We determined the predictive value of 6-MMPR concentrations for the development of these adverse reactions during the first 20 weeks of treatment.

\section{Methods}

The cohort study consisted of the first 270 IBD patients starting thiopurine treatment as part of the Dutch randomised-controlled multi-center TOPIC trial (ClinicalTrials.gov NCT00521950). Blood samples for metabolite assessment were collected one week after initiation. Hepatotoxicity was defined by alanine aminotransaminase, aspartate aminotransaminase or alkaline phosphatase elevations up to at least twice the upper limit of normal.

\section{Results}

Forty-seven patients (17\%) presented signs of hepatotoxicity during the first 20 weeks of thiopurine treatment. A 6-MMPR threshold level at week 1 of $3,615 \mathrm{pmol} / 8 \times 10^{8}$ erythrocytes was defined. 6-MMPR concentrations above the threshold correlated with gastrointestinal complaints (i.e. nausea, vomiting and anorexia; $\mathrm{OR}=2.4(95 \% \mathrm{Cl}$ : $1.4-4.3))$ and general malaise $(\mathrm{OR}=2.0$ (95\% Cl: 1.1-3.7). Analysis of patients on stable thiopurine dose $(n=174)$ showed that those exceeding the 6-MMPR threshold were at increased risk of hepatotoxicity, OR $3.8(95 \% \mathrm{Cl}$ : 1.8-8.0).

A predictive algorithm was developed based on the relevant determinants age, gender, $\mathrm{BMI}$ and the 6-MMPR threshold to assess the risk of hepatotoxicity (AUC 0.83 ( $95 \% \mathrm{Cl}: 0.75-0.91)$ ).

\section{Conclusions}

Patients at risk for thiopurine-induced hepatotoxicity, gastrointestinal complaints and general malaise can be identified by assessment of the 6-MMPR concentration one week after initiation, allowing optimising thiopurine treatment in IBD. 


\section{Introduction}

The conventional immunosuppressive thiopurine drugs azathioprine (AZA) and 6-mercaptopurine (6MP) are commonly used in the management of both moderate-tosevere Crohn's disease (CD) and ulcerative colitis (UC), and have proven efficacy in inducing, but particularly in maintaining remission of these inflammatory bowel diseases (IBD). ${ }^{1-3}$

Neither AZA nor 6MP has intrinsic pharmacological properties and both drugs need to undergo various intracellular transformations to form active metabolites of which 6-thioguanine nucleotides (6-TGN) and 6-methylmercaptopurine ribonucleotides (6-MMPR) are considered the most important. Thiopurine S-methyltransferase (TPMT) plays a pivotal role in the balance of 6-TGN and the methylated 6-MMPR metabolite formation, but the enzyme activity shows high interindividual variation due to genetic diversity (Figure 7.1). ${ }^{4}$

Up to a quarter of the patients discontinue thiopurine therapy due to adverse events within the first 3 months of treatment. ${ }^{5}$ The most common adverse drug reactions prompting to discontinuation are gastrointestinal intolerance (i.e. nausea and vomiting) and hepatotoxicity. ${ }^{5,6}$

Thiopurine-induced hepatotoxicity may occur in up to $10 \%$ of patients, most frequently during the first months of therapy and is therefore one of the most common and limiting adverse events in IBD often resulting in therapeutic failure. ${ }^{7-9}$ Dose-related hepatotoxicity induced by thiopurine drugs, has been related to high steady-state 6-MMPR metabolite concentrations. ${ }^{10-12}$ In a paediatric study, steady-state 6-MMPR metabolite concentrations $>5,700 \mathrm{pmol} / 8 \times 10^{8}$ red blood cells (RBC) have been associated with an increased risk of developing hepatotoxicity, which is in accordance with other studies. ${ }^{10,11,13}$ In adult IBD patients elevated steady-state 6-MMPR metabolite concentrations have also been related to hepatotoxicity and subsequent thiopurine withdrawal. ${ }^{5}$ On the other hand, hepatotoxicity may also be observed in patients with low 6-MMPR concentrations, therefore the precise role of 6-MMPR levels in predicting hepatotoxicity remains unclear. ${ }^{14,15}$

Early identification of patients at risk of thiopurine-related adverse events is important in order to improve thiopurine effectiveness in IBD, because alternative therapeutic options are limited.

The main objective of our study in IBD patients was to assess the predictive value of 6-MMPR metabolite concentrations, determined one week after thiopurine initiation, for the development of thiopurine-induced hepatotoxicity during the first 20 weeks of therapy. We also evaluated the association between 6-MMPR metabolites and the occurrence of gastrointestinal complaints and general malaise. 


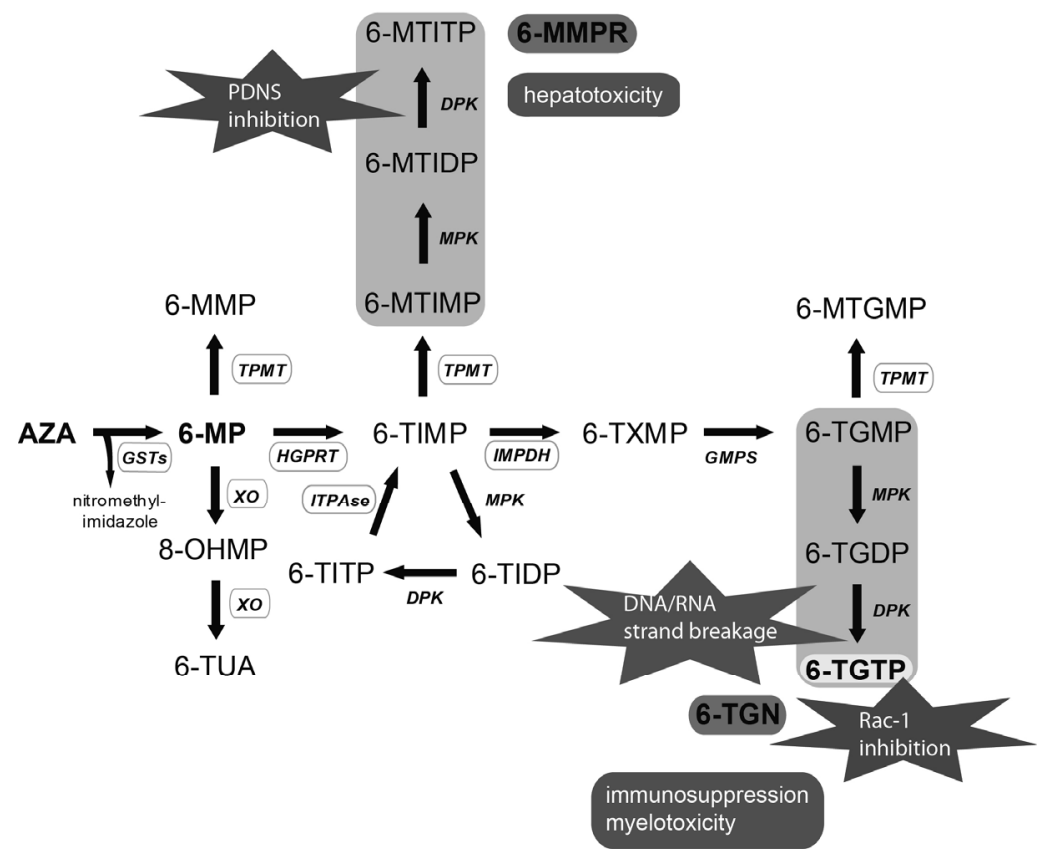

Figure 7.1 Proposed thiopurine metabolism.

AZA, azathioprine; 6MP, 6-mercaptopurine; 6-MMP, 6-methylmercaptopurine; 8-OHMP, 8-hydroxy-6-mercaptopurine; 6-TUA, 6-thiouric acid; 6-MTIMP, 6-methylthioinosine monophosphate; 6-MTIDP, 6-methylthioinosine diphosphate; 6-MTITP, 6-methylthioinosine triphosphate; 6-TIMP, 6-thioinosine monophosphate; 6-TIDP, 6-thioinosine diphosphate; 6-TITP, 6-thioinosine triphosphate; 6-TXMP, 6-thioxanthosine monophosphate; 6-TGMP, 6-thioguanine monophosphate; 6-TGDP, 6-thioguanine diphosphate; 6-TGTP, 6-thioguanine triphosphate; 6-MTGMP, 6-methylthioguanine monophosphate; 6-TG, 6-thioguanine; 6-MTG, 6-methylthioguanine; $\mathrm{XO}$, xanthine oxidase; GST, glutathione S-transferase; TPMT, thiopurine S-methyltransferase; HGPRT, hypoxanthine phosphoribosyl transferase; IMPDH, inosine monophosphate dehydrogenase; GMPS, guanosine monophosphate synthetase; MPK, monophosphate kinase; DPK, diphosphate kinase; ITPase, inosine triphosphate pyrophosphatase; PDNS, purine de novo synthesis; DNA, deoxyribonucleic acid; RNA, ribonucleic acid. 6-MTIMP, 6-MTIDP and 6-MTITP together form the 6-methylmercaptopurine ribonucleotides (6-MMPR). 6-TGMP, 6-TGDP and 6-TGTP together form the 6-thioguaninenucleotides (6-TGN). Enzymes encoded by genes that are subject to known genetic polymorphisms are circled in grey. (Adapted from Derijks et al. ${ }^{4}$ ).

\section{Materials and methods}

\section{Study design and outcome definition}

The study was performed among the first 270 consecutive included patients of the TOPIC study, a large randomised-controlled trial in 769 IBD patients evaluating the 
efficacy and cost-effectiveness of pre-treatment TPMT genotyping (Figure 7.2). The TOPIC study was approved by the Medical Ethics Committees of the participating centres. Written informed consent was obtained from all participating patients. The study is registered at clinicaltrials.gov, as Cost-Effectiveness of TPMT Pharmacogenetics (TOPIC), trial number NCT00521950. For a detailed description of the TOPIC trial, we refer to Coenen et al.. ${ }^{16}$

Briefly, patients were assessed for eligibility by their gastroenterologist. The inclusion criteria of the TOPIC trial were: age $>18$ years, diagnosis of IBD and the indication for AZA or 6MP treatment. Exclusion criteria were: previous use of AZA or 6MP, treatment with allopurinol, known TPMT enzyme activity or genotype, current pregnancy, and a leukocyte count $<3.0 \times 10^{9} /$, liver test abnormalities (alanine transaminase (ALAT), aspartate transaminase (ASAT), alkaline phosphatase (AP), and/or gamma glutamate transpeptidase $\geq$ twice the upper limit of the normal value), and impaired renal function (serum creatinine level $\geq$ twice the upper limit of the normal value) at baseline.

Patients meeting the inclusion criteria were randomly assigned to either standard thiopurine treatment (AZA $2-2.5 \mathrm{mg} / \mathrm{kg} /$ day or $6 \mathrm{MP} 1-1.5 \mathrm{mg} / \mathrm{kg} /$ day) or a dose regimen based on the pre-treatment determined TPMT genotype. Patients of the intervention group carrying a genetic TPMT variant were treated with $50 \%$ (TPMT heterozygous) or $0-10 \%$ (TPMT homozygous) of the standard thiopurine dose, according to the guideline of the Dutch Pharmacogenetics Working Group. ${ }^{17}$ All patients were clinically followed for $20( \pm 6)$ consecutive weeks after treatment initiation. Safety was evaluated by measurement of haematological and biochemical parameters one week before initiation and at least at weeks 1, 2, 4, 6, 8 and 20 after initiation of thiopurine therapy. For the present study, blood samples for 6-MMPR and 6-TGN measurements were collected one week (i.e. $~ 7 \pm 1$ day) after thiopurine initiation (T1). The thiopurine metabolite concentrations were measured after the follow-up period.

Thiopurine dose adjustments were defined as thiopurine dose escalation or reduction. Any decision regarding dose adjustments or discontinuation of thiopurine therapy due to myelotoxicity, hepatotoxicity, (acute) pancreatitis and other adverse events or thiopurine intolerance were made by the responsible physician and was subsequently recorded.

Hepatotoxicity, considered as signs of hepatocellular injury, was defined by elevation from baseline of at least one of the liver enzymes ALAT, ASAT or AP, up to at least twice the upper limit of the reference values (2N). Nausea, vomiting and/or anorexia were considered as gastrointestinal complaints based on patient reports.

Thiopurine-induced hepatotoxicity, gastrointestinal complaints and general malaise were evaluated in relation to 6-MMPR and 6-TGN concentrations assessed at T1. Therefore, a prior-to-treat TPMT genotype-guided dose regimen in the course of the TOPIC trial did not interfere with our primary outcome.

The design of the study and patient selection is depicted in Figure 7.2. 


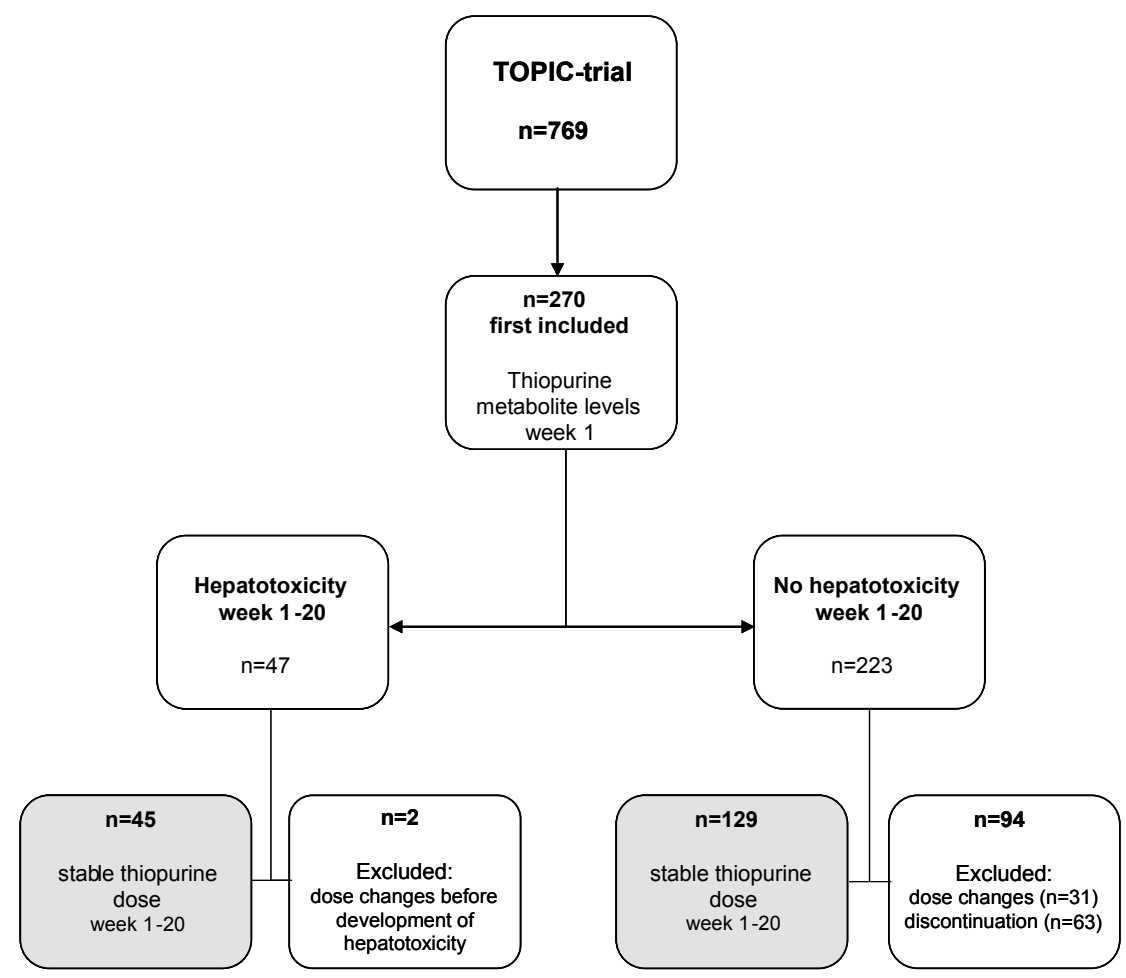

Signs of hepatotoxicity?

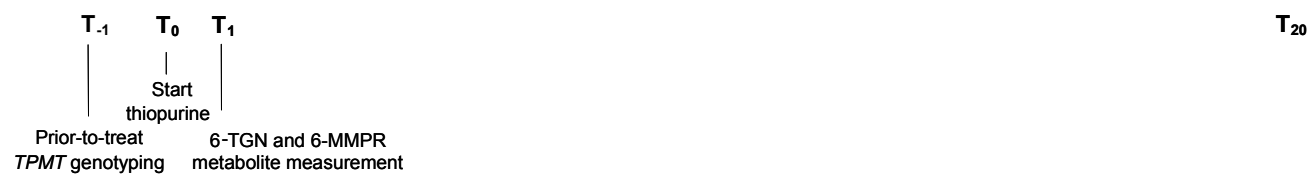

Figure 7.2 Study design and time-line within the TOPIC-trial.

\section{Measurement of 6-MMPR and 6-TGN metabolite concentrations}

6-MMPR and 6-TGN concentrations were determined in erythrocytes by a modified high performance liquid chromatography (HPLC) method of Lennard and Singleton, as published previously by Derijks et al.. ${ }^{18}$ The lower-limits of quantification for 6-TGN and 6-MMPR metabolite levels were $40 \mathrm{pmol} / 8 \times 10^{8} \mathrm{RBC}$ and $300 \mathrm{pmol} / 8 \times 10^{8} \mathrm{RBC}$, respectively.

Blood samples for thiopurine metabolite measurement were immediately stored in the refrigerator $\left(2-8^{\circ} \mathrm{C}\right)$ in order to secure metabolite stability and subsequently sent to the Laboratory of the Department of Clinical Pharmacy and Toxicology of the Orbis Medical 
Centre (Sittard-Geleen, The Netherlands), where the erythrocytes were washed, counted and stored at $-20^{\circ} \mathrm{C}$ until required.

\section{Measurement of TPMT enzyme activity}

TPMT enzyme activity was measured in red blood cells using a HPLC method as described previously by Ford and colleagues. ${ }^{19,20}$ Blood samples for TPMT enzyme activity measurement were collected one week before thiopurine initiation and were immediately stored at $-80^{\circ} \mathrm{C}$ until required. TPMT enzyme activity measurement was performed after the follow-up period of 20 weeks of the TOPIC trial. ${ }^{16}$

\section{Data analysis}

Descriptive data analysis was performed by percentages for categorical variables and by median values and ranges for continuous characteristics. Box-and-whisker plots served for visual presentation. Differences in patient characteristics were evaluated using the Pearson Chi-square test; for differences in metabolite concentrations the MannWhitney $U$ test or Kolmogorov-Smirnov $Z$ test were applied, when appropriate. P-values of less than 0.05 (two-sided) were considered to be statistically significant. SPSS for Windows version 20.0 was used for all data analysis.

The predictive threshold value of the thiopurine metabolites on the occurrence of hepatotoxicity was evaluated in patients on stable thiopurine dose. Patients with thiopurine dose adjustments or who discontinued thiopurine therapy during the followup period of 20 weeks not related to hepatotoxicity were excluded from analysis. To explore the discriminative power of 6-MMPR levels regarding hepatotoxicity Receiver Operating Characteristic (ROC) curves were generated to plot sensitivity and false positive rates (1-specificity). The areas under the ROC curves (AUC) and 95\% confidence interval $(95 \% \mathrm{Cl})$ were used to assess the predictive performance.

The predictive power of the metabolites for the occurrence of hepatotoxicity was also studied by logistic regression modelling taking covariates into account such as age, gender, disease type (CD/UC), body weight / body mass index (BMI) and IBD medication (systemic corticosteroids, thiopurine type, thiopurine dose and concurrent use of drugs interfering with thiopurine metabolism (i.e. mesalazine (5-ASA), anti-TNF- $\alpha$ therapy (infliximab (IFX), adalimumab (ADA), when appropriate. The results are expressed as odds ratios (OR) with $95 \% \mathrm{Cl}$.

\section{Results}

\section{Patient population}

In total, 270 IBD patients (117 males, 153 females) with a median age of 40 years (range 19-81) were analysed. CD and UC was diagnosed in 149 (55\%) and 119 patients (45\%); 
two patients were diagnosed with indeterminate colitis (IBD-UC). Baseline patient characteristics of the study population are given in Table 7.1.

Table 7.1 Baseline characteristics of the study population.

\begin{tabular}{|c|c|c|c|c|}
\hline & All & Hepatotoxicity & No hepatotoxicity & $\mathrm{p}$-value \\
\hline \multicolumn{5}{|l|}{ Patients } \\
\hline Patient number (n) & 270 & 47 & 223 & \\
\hline $\begin{array}{l}\text { Age in years (median, range) } \\
\text { Gender }\end{array}$ & $40(19-81)$ & $51(20-75)$ & $37(19-81)$ & $<0.001$ \\
\hline male (n) & 117 & 29 & 88 & 0.005 \\
\hline Female (n) & 153 & 18 & 135 & \\
\hline $\begin{array}{l}\text { Body weight T0 in } \mathrm{kg} \text { (median, range) } \\
\text { Body Mass Index in } \mathrm{kg} / \mathrm{m}^{2} \\
\text { (median, range) } \\
(\mathrm{n}=244 \text {; missing } \mathrm{n}=26 ; 4 \text { / 22) }\end{array}$ & $\begin{array}{l}72(40-160) \\
24(17-50)\end{array}$ & $\begin{array}{l}82(50-160) \\
27(19-38)\end{array}$ & $\begin{array}{l}71(40-121) \\
23(17-50)\end{array}$ & $\begin{array}{l}<0.001 \\
<0.001\end{array}$ \\
\hline \multicolumn{5}{|l|}{ IBD } \\
\hline Crohn's disease $(n)^{*}$ & 149 & 23 & 126 & $0.311^{*}$ \\
\hline Ulcerative colitis $(n)^{*}$ & 119 & 24 & 95 & \\
\hline Indeterminate colitis (IBD-UC) (n) & 2 & & & \\
\hline $\begin{array}{l}\text { Disease duration in years } \\
\text { (median; range) (missing } n=1 / 0 \text { ) }\end{array}$ & $1.4(0-50)$ & $1.4(0-32)$ & $1.4(0-50)$ & 0.332 \\
\hline $\begin{array}{l}\text { TPMT enzyme activity (week -1) } \\
\text { (mg 6-MTG/mmol } \mathrm{Hb} / \mathrm{h}) ; \text { mean }(95 \% \mathrm{Cl})\end{array}$ & $90.2(83.3-94.8)$ & $89.1(83.3-94.8)$ & $90.4(87.4-93.4)$ & 0.698 \\
\hline \multicolumn{5}{|l|}{ Medication } \\
\hline Azathioprine (n) & 183 & 25 & 158 & 0.019 \\
\hline 6-mercaptopurine (n) & 87 & 22 & 65 & \\
\hline Azathioprine (n) & 183 & 25 & 158 & \\
\hline AZA dose in mg (median, range) & $150(25-250)$ & $150(50-250)$ & $150(25-250)$ & 0.426 \\
\hline AZA dose in $\mathrm{mg} / \mathrm{kg}$ (median, range) & $2.2(0.3-3.1)$ & $2.1(0.6-2.4)$ & $2.2(0.3-3.1)$ & 0.446 \\
\hline 6-mercaptopurine $(n)$ & 87 & 22 & 65 & \\
\hline $6 \mathrm{MP}$ dose in $\mathrm{mg}$ (median, range) & $75(50-150)$ & $100(50-150)$ & $75(50-150)$ & 0.002 \\
\hline $6 \mathrm{MP}$ dose in $\mathrm{mg} / \mathrm{kg}$ (median, range) & $1.2(0.6-2.0)$ & $1.2(0.6-1.5)$ & $1.2(0.6-2.0)$ & 0.625 \\
\hline \multicolumn{5}{|l|}{ Concurrent IBD medication ( $\mathrm{n}$, yes / no) } \\
\hline 5 -ASA & $155 / 115$ & $28 / 19$ & $127 / 96$ & 0.741 \\
\hline Corticosteroids & $223 / 47$ & $41 / 6$ & 182 / 41 & 0.356 \\
\hline Biologicals (IFX, ADA) & $16 / 254$ & $4 / 43$ & 12 / 211 & 0.493 \\
\hline
\end{tabular}

\section{Thiopurine related adverse drug events}

Eighty-six of 270 patients (32\%) discontinued thiopurine therapy within the first 5 months due to thiopurine intolerance or adverse drug events, of whom $84 \%$ (72 of 86) discontinued within the first 8 weeks. The median time of thiopurine withdrawal was 4 weeks (1-24). No difference in thiopurine withdrawal rate was observed between patients treated with 6MP and AZA ( $p=0.142)$.

During the follow-up period of 20 weeks, 47 of 270 patients (17\%) developed hepatotoxicity after a median time of 4 weeks (1-22). Forty-four (94\%) of these patients 
showed an elevation of serum ALAT $>2 \mathrm{~N}$. In 40 of 47 patients (85\%) signs of hepatotoxicity were observed within the first 8 weeks after thiopurine initiation. Fortyeight per cent (130 of 270) of the patients reported gastrointestinal complaints (i.e. nausea (40\%), vomiting (15\%) and/or anorexia (21\%)) during the follow-up period. General malaise was reported by $27 \%$ (73 of 270 ) of the patients (Table 7.2 ).

Table 7.2 Most common thiopurine-limiting adverse reactions during the follow-up period of the first 20 weeks of treatment $(n=270)$.

\begin{tabular}{ll}
\hline Adverse reaction & Cumulative incidence, $\mathrm{n}(\%)$ \\
\hline Hepatotoxicity & \\
ALAT, ASAT or AP $\geq 2 \mathrm{~N}$ & $47(17 \%)$ \\
ALAT and ASAT and/or AP $\geq 2 \mathrm{~N}$ & $44(16 \%)$ \\
& \\
Gastrointestinal complaints & \\
Nausea, vomiting and/or anorexia & $130(48 \%)$ \\
Nausea & $108(40 \%)$ \\
Vomiting & $41(15 \%)$ \\
Anorexia & $57(21 \%)$ \\
General malaise & $73(27 \%)$ \\
\hline
\end{tabular}

\section{Thiopurine metabolite concentrations at week 1 and patient characteristics}

6-MMPR concentrations at week 1 were higher in females (compared to males), in patients treated with $6 \mathrm{MP}$ (compared to AZA) and in patients with a BMI $>25 \mathrm{~kg} / \mathrm{m}^{2}$. Concomitant treatment of systemic corticosteroids, 5-ASA and anti-TNF (i.e. IFX, ADA) did not affect 6-MMPR concentrations at week 1 (Table 7.3).

\section{Thiopurine-induced hepatotoxicity}

Hepatotoxicity was more frequently observed in patients treated with 6MP, when compared to AZA (25\% (22/87) versus 14\% (25/183), OR 2.1 (95\%Cl: 1.1-4.1)). When compared to patients without signs of hepatotoxicity, age, bodyweight (T0), body mass index (BMI) and 6MP dose were higher in the hepatotoxicity group. Furthermore, hepatotoxicity occurred more in males, than in females (33\% (29/88) versus $13 \%$ (18/135), $p=0.005)$. Hepatotoxicity rates were comparable for patients with and without concomitant anti-TNF, mesalazine or corticosteroid treatment (Table 7.1).

Median 6-MMPR metabolite levels at week 1 were significantly higher in patients who developed hepatotoxicity during week 1-20, when compared to patients who did not: $3,111(300-15,691)$ versus $1,811 \mathrm{pmol} / 8 \times 10^{8}$ RBC $(300-11,230)$, respectively $(p<0.001$, Figure 7.3); 6-TGN metabolite concentrations did not differ between the patient groups (147 (40-454) versus $154(40-607) \mathrm{pmol} / 8 \times 10^{8} \mathrm{RBC}$, respectively $\left.(p=0.952)\right)$. The 
6-MMPR/6-TGN ratio at week 1 was significantly higher in patients who developed hepatotoxicity $(22(1.5-74)$ versus $13(0.8-84), p<0.001)$.

Prior-to-treat assessed TPMT enzyme activity was comparable between both patient groups $(p=0.698)$.

Table 7.3 Thiopurine metabolite concentrations at week 1 for different patient characteristics.

\begin{tabular}{|c|c|c|c|c|}
\hline & & Patient number $(n)$ & T1 6-TGN ${ }^{*}$ & T1 6-MMPR ${ }^{*}$ \\
\hline All patients & & 270 & & \\
\hline \multicolumn{5}{|l|}{ Gender } \\
\hline male & & 117 & $149(40-528)$ & $1,637(300-10,009)$ \\
\hline female & & 153 & $155(40-607)$ & $2,606(300-15,691)$ \\
\hline$p$-value & & & 0.285 & 0.002 \\
\hline \multicolumn{5}{|l|}{ IBD } \\
\hline Crohn's disease & & 149 & $161(40-564)$ & $2,173(300-11,230)$ \\
\hline Ulcerative colitis & & 119 & $130(42-607)$ & $1,838(300-15,691)$ \\
\hline \multicolumn{5}{|l|}{$\mathrm{n}=2$ missing (IBD-UC) } \\
\hline $\mathrm{p}$-value & & & 0.031 & 0.861 \\
\hline \multicolumn{5}{|l|}{ Thiopurine type } \\
\hline AZA & & 183 & $133(40-607)$ & $1,798(300-11,965)$ \\
\hline $6 \mathrm{MP}$ & & 87 & $195(44-564)$ & $2,991(300-15,691)$ \\
\hline $\mathrm{p}$-value & & & $<0.00001$ & 0.001 \\
\hline \multicolumn{5}{|l|}{ Body Mass Index $\left(\mathrm{kg} / \mathrm{m}^{2}\right)$} \\
\hline $\mathrm{BMI}<25$ & & 146 & $158(50-528)$ & $1,816(300-11,230)$ \\
\hline $\mathrm{BMI}>25$ & & 98 & $134(40-607)$ & $2,665(300-15,691)$ \\
\hline p-value & & & 0.142 & 0.005 \\
\hline \multicolumn{5}{|l|}{$\mathrm{n}=26$ missing (length) } \\
\hline \multicolumn{5}{|c|}{ Concurrent IBD medication at baseline } \\
\hline \multirow[t]{3}{*}{ 5-ASA } & yes & 155 & $151(40-607)$ & $1,812(300-15,691)$ \\
\hline & no & 115 & $155(40-528)$ & $2,244(300-11,042)$ \\
\hline & p-value & & 0.440 & 0.272 \\
\hline \multirow[t]{3}{*}{ Corticosteroids } & yes & 223 & $139(40-528)$ & $2,013(300-15,691)$ \\
\hline & no & 47 & $179(40-607)$ & $1,849(300-11,230)$ \\
\hline & p-value & & 0.005 & 0.955 \\
\hline \multirow[t]{3}{*}{ Anti-TNF- $\alpha$ (IFX, ADA) } & yes & 16 & $159(51-459)$ & $2,059(300-10,009)$ \\
\hline & no & 254 & $151(40-607)$ & $1,942(300-15,691)$ \\
\hline & $p$-value ${ }^{\#}$ & & 0.990 & 0.595 \\
\hline
\end{tabular}

*6-TGN and 6-MMPR concentrations ( $\mathrm{pmol} / 8 \times 10^{8} \mathrm{RBC}$ ) are presented as median values and ranges

\# Kolmogorov-Smirnov Z test.

In order to assess the predictive value for 6-MMPR at week 1 for hepatotoxicity, patients with dose adjustments ( $n=33 ; n=2$ developed afterwards hepatotoxicity) and/or discontinuation ( $n=63$ ) not related to hepatotoxicity were excluded (Figure 7.2). Forty-five patients of the 174 remaining patients developed hepatotoxicity during week 1-20.

The area under the ROC curve of the continuous 6-MMPR concentration for the occurrence of hepatotoxicity during week $1-20$ was $0.65(95 \% \mathrm{Cl}: 0.55-0.75 ; p=0.002)$. 


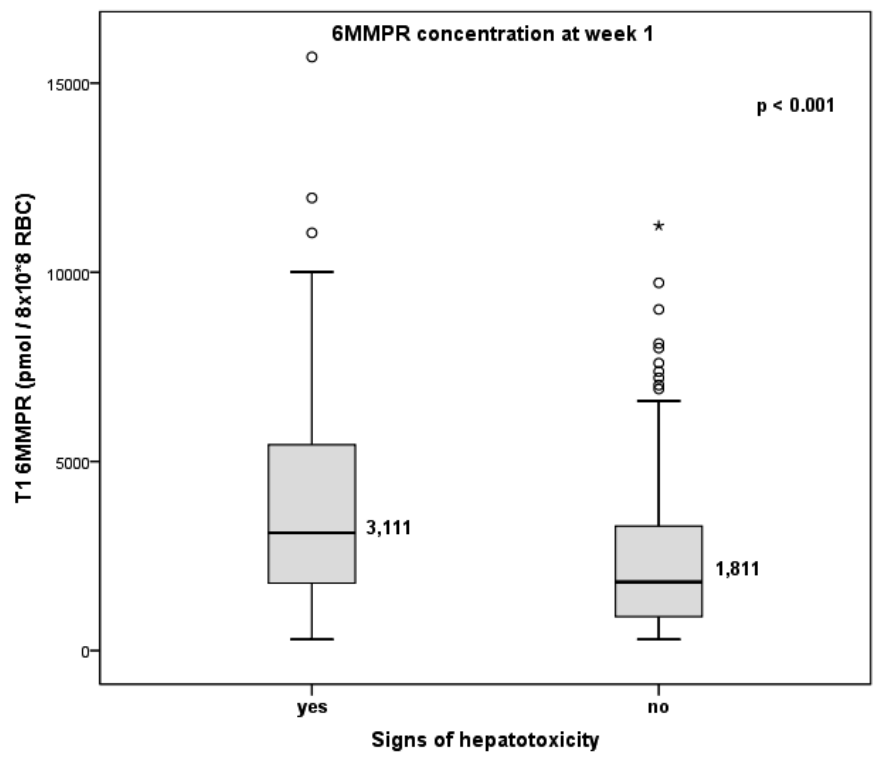

Figure 7.3 Box-whisker plots of the 6-MMPR concentrations at week 1 for the occurrence of hepatotoxicity $(n=47)$ reported during the follow-up period of the first 20 weeks of thiopurine therapy in IBD patients $(n=270)$.

Based on the quartile analysis of the $T 16-M M P R$ concentrations, we defined a 6-MMPR threshold value of $3,615 \mathrm{pmol} / 8 \times 10^{8} \mathrm{RBC}$ to predict hepatotoxicity, using the lower limit of the upper quartile $\left(75^{\text {th }}\right.$ percentile). The associated specificity and sensitivity rates for hepatotoxicity were $84 \%$ and $42 \%$, respectively.

At the $T 1$ 6-MMPR threshold of 3,615 pmol $/ 8 \times 10^{8} \mathrm{RBC}$, patients with elevated $T 1$

6-MMPR concentrations were at increased risk of hepatotoxicity: $48 \%$ (19/40) of the patients above the threshold developed hepatotoxicity versus $19 \%(26 / 134)$ below the threshold (OR 3.8 (95\% Cl: 1.8-8.0); $\mathrm{p}<0.001)$. The area under the ROC curve of the $T 16$ MMPR threshold level for the development of hepatotoxicity was $0.63(95 \% \mathrm{Cl}$ : 0.53-0.73; $p=0.010)$. An area under the ROC curve of 0.61 (95\% Cl: $0.52-0.71 ; p=0.016)$ and an OR of $2.9(95 \% \mathrm{Cl}: 1.5-5.7 ; \mathrm{p}=0.001)$ were found, when all 270 patients were evaluated.

\section{Multivariable prediction model for thiopurine-induced hepatotoxicity}

Univariate regression analysis of patients on stable thiopurine therapy showed that age, gender, bodyweight and BMI at baseline and thiopurine type were relevant determinants for the development of hepatotoxicity during thiopurine therapy. Age and $\mathrm{BMI}$ appeared to be the best discriminative parameters showing areas under the ROC curves of 0.75 (95\% Cl: $0.67-0.83 ; \mathrm{p}<0.001)$ and $0.77(95 \% \mathrm{Cl}: 0.69-0.85 ; \mathrm{p}<0.001)$, 
respectively. Age dichotomised at 50 years (median value of the hepatotoxicity group) and $\mathrm{BMI}$ at $25 \mathrm{~kg} / \mathrm{m}^{2}$, to distinct normal weight and overweight, yielded ORs and predictive characteristics for the development of hepatotoxicity, as presented in Table 7.4. A multivariable regression analysis of the 16 categories was performed based on age $>50$ years, $\mathrm{BMI}>25 \mathrm{~kg} / \mathrm{m}^{2}$, male gender and the $T 16-\mathrm{MMPR}$ threshold parameter. For comparison, females $<50$ years with a BMI $<25 \mathrm{~kg} / \mathrm{m}^{2}$ and a $T 1$ 6-MMPR concentration $<3,615 \mathrm{pmol} / 8 \times 10^{8} \mathrm{RBC}$ were used as the reference group. Fifteen patients ( $n=3$ with hepatotoxicity) were excluded from analysis, due to missing data of length of these patients. Multivariable regression analysis of the various categories including 159 patients ( $n=42$ with hepatotoxicity) revealed that males irrespective of age (age $>50$ years $n=7$ or age $<50$ years $n=4$ ) with a $B M l>25 \mathrm{~kg} / \mathrm{m}^{2}$ and a $T 16-M M P R$ $>3,615 \mathrm{pmol} / 8 \times 10^{8} \mathrm{RBC}$ were at the highest risk, as these patients all developed hepatotoxicity. Then, females aged $>50$ years with a $\mathrm{BMl}>25 \mathrm{~kg} / \mathrm{m}^{2}$ and $T 16-\mathrm{MMPR}$ concentration above the threshold were at highest risk to develop hepatotoxicity ( 3 of 4 patients, OR 36.0 (95\% Cl: $2.8-462)$ ), followed by females (3 of 5) and males (4 of 8 ) older than 50 years with a $\mathrm{BMI}>25 \mathrm{~kg} / \mathrm{m}^{2}$ and a $T 16-\mathrm{MMPR}$ level below the threshold (OR 18.0 (95\% Cl: 2.1-153) and OR 12.0 (95\% $\mathrm{Cl}:$ 1.9-74), respectively). Patients not meeting the described criteria were at a lower risk of developing hepatotoxicity. The area under the ROC curve for the obtained predicted probabilities based on the four defined categories was $0.83(95 \% \mathrm{Cl}: 0.75-0.91 ; \mathrm{p}<0.00001)$. Figure 7.4 presents the area under the ROC curves for the 6-MMPR concentration, the predictive threshold and the predicted probabilities based on the relevant determinants for the development of thiopurine-induced hepatotoxicity.

Table 7.4 Univariate analyses of the relevant independent determinants for the development of hepatotoxicity in patients on stable thiopurine dose $(n=174)$.

\begin{tabular}{lllll}
\hline Determinant & Odds ratio $(95 \% \mathrm{Cl})$ & AUC ROC $(95 \% \mathrm{Cl})$ & Specificity & Sensitivity \\
\hline Age $>50$ years & $4.5(2.2-9.3)$ & $0.67(0.57-0.77)$ & 0.78 & 0.56 \\
$\mathrm{BMI}>25 \mathrm{~kg} / \mathrm{m}^{2 \#}$ & $4.6(2.2-9.7)$ & $0.68(0.59-0.78)$ & 0.72 & 0.64 \\
Gender $($ male) & $2.5(1.3-5.1)$ & $0.61(0.52-0.71)$ & 0.60 & 0.62 \\
T1 6-MMPR $>3,615$ pmol $^{*}$ & $3.8(1.8-8.0)$ & $0.63(0.53-0.73)$ & 0.84 & 0.42 \\
\hline
\end{tabular}

* Thiopurine metabolite concentration in pmol/8x10 RBC; $n=159$ ( $n=42$ with hepatotoxicity and $n=117$ without hepatotoxicity), because the length of 15 patients was missing.

Signs of hepatotoxicity resulted often in thiopurine discontinuation: $77 \%(23 / 30)$ of the patients with hepatotoxicity discontinued, compared to $15 \%(22 / 144)$ of patients who continued therapy (OR $18.2(95 \% \mathrm{Cl}: 7.0-47.6)$. In addition, in 53\% (10/19) of the patients with hepatotoxicity thiopurine dose was reduced, compared to $23 \%$ of patients (35/155) without hepatotoxicity, OR 3.8 (95\%Cl: 1.4-10.1).

Signs of hepatotoxicity correlated well with reported gastrointestinal complaints (OR $2.6(95 \% \mathrm{Cl}: 1.3-5.2))$, but not with general malaise $(\mathrm{p}=0.147)$. 


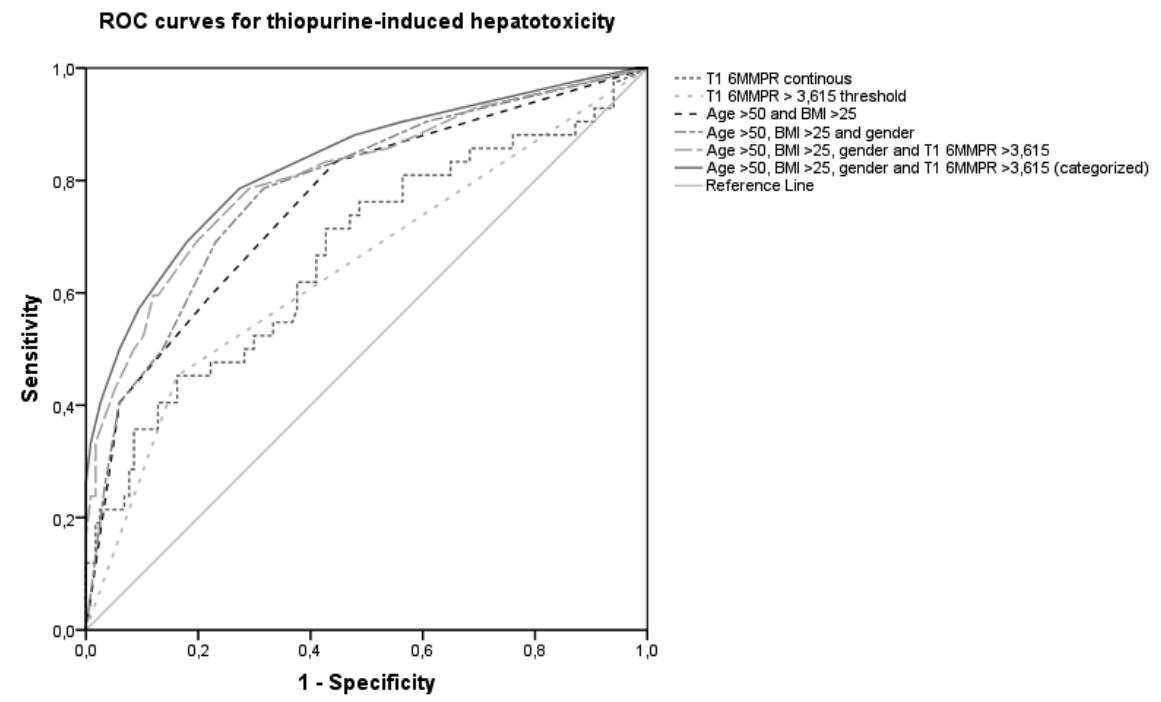

Figure 7.4 Receiver operating characteristic curves for 6-MMPR metabolite concentrations at week 1, the threshold parameter and the predicted probabilities based on the relevant determinants age, BMI and gender.

\section{Gastrointestinal complaints and general malaise}

In patient groups who did or did not report gastrointestinal complaints (i.e. nausea, vomiting and/or anorexia) and general malaise, no difference between age and bodyweight at baseline was found. BMI $\left(\mathrm{T}_{0}\right)$ was higher in patients who reported gastrointestinal complaints, when compared to patients without (median 25 (17-50) resp. $\left.23(17-40) \mathrm{kg} / \mathrm{m}^{2}, p=0.043\right)$, but not for general malaise $(p=0.195)$. Females reported more gastrointestinal complaints than males (OR 1.8 (95\% $\mathrm{Cl}$ : 1.1-2.9); no differences were observed between patients with $C D$ or UC. The incidence of gastrointestinal complaints was higher in patients treated with $6 \mathrm{MP}$, when compared to AZA (60\% (52/87) versus 43\% (78/183); OR 2.0 (95\% $\mathrm{Cl}: 1.2-3.4)$, but not for general malaise $(p=0.058)$.

Thiopurine discontinuation rate was higher in patients who reported gastrointestinal complaints (OR 2.6 (95\% Cl: 1.5-4.4) and general malaise (OR 3.4 (95\% Cl: 1.9-5.9).

At week 1, higher 6-MMPR levels were found in patients with gastrointestinal complaints, when compared to patients without these complaints $(2,599(300-15,691)$ versus $\left.1,731(300-11,965) \mathrm{pmol} / 8 \times 10^{8} \mathrm{RBC}, \mathrm{p}=0.009\right)$. 6-TGN concentrations were comparable for both patient groups $(p=0.291)$, consequently the 6-MMPR/6-TGN ratio at week 1 was higher in patients with gastrointestinal complaints (18 (0.9-84) versus 12 $(0.8-78), p=0.018)$. 
In addition, 6-MMPR levels and 6-MMPR/6-TGN ratio at week 1 were higher in patients who reported general malaise, compared to patients without these complaints $(2,480$ (300-15,691) versus $1,848(300-11,965) \mathrm{pmol} / 8 \times 10^{8} \mathrm{RBC} ; \mathrm{p}=0.030$, and 20 (0.9-71) versus 13 (0.8-84); $p=0.016$, respectively).

TPMT enzyme activity $\left(T_{-1}\right)$ was comparable between patients with and without gastrointestinal complaints or general malaise $(p=0.424$ and $p=0.650$, respectively).

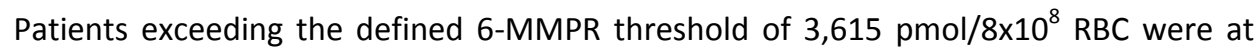
increased risk to experience gastrointestinal complaints (OR 2.4 (95\% Cl: 1.4-4.3) and general malaise (OR 2.0 (95\% Cl: 1.1-3.7).

The areas under the ROC curves of the continuous 6-MMPR concentrations and the defined threshold for the occurrence of gastrointestinal complaints were $0.59(95 \% \mathrm{Cl}$ : $0.53-0.66 ; p=0.009)$ and $0.58(95 \% \mathrm{Cl}: 0.51-0.65 ; \mathrm{p}=0.024)$, respectively, and 0.59 (95\% $\mathrm{Cl}: 0.51-0.66, p=0.030)$ and $0.57(95 \% \mathrm{Cl}: 0.49-0.65, p=0.079)$, respectively, for general malaise. The predicted probabilities obtained from multivariable regression analysis of the dichotomised BMI at $25 \mathrm{~kg} / \mathrm{m}^{2}$, gender, thiopurine type and the 6-MMPR threshold concentration for the occurrence of gastrointestinal complaints revealed an area under the ROC curve of $0.63(95 \% \mathrm{Cl}: 0.56-0.70 ; \mathrm{p}=0.001)$ and $0.59(95 \% \mathrm{Cl}$ : $0.51-0.68, p=0.024$ ) for general malaise.

\section{Discussion}

In this prospective study we developed for the first time a highly accurate prediction model for thiopurine-induced hepatotoxicity, based on 6-MMPR metabolite concentrations assessed at week one and the relevant patient-related determinants age, gender and BMI. Thiopurine-associated hepatotoxicity occurred in more than $80 \%$ of the patients during the first 8 weeks of treatment and strongly correlated with thiopurine withdrawal and reduction of thiopurine dose.

Steady-state thiopurine metabolite concentrations are generally reached after approximately four weeks of therapy. ${ }^{18}$ Dubinsky and colleagues reported in a prospective study of paediatric IBD patients a 3-fold increased risk of hepatotoxicity for steady-state 6-MMPR concentrations above $5,700 \mathrm{pmol} / 8 \times 10^{8} \mathrm{RBC}^{10}$

In the present study, adult patients with 6-MMPR concentrations exceeding the defined threshold of $3,615 \mathrm{pmol} / 8 \times 10^{8} \mathrm{RBC}$ at week one were approximately 4 times more at risk of hepatotoxicity during the first 20 weeks of treatment. The predictive power of the 6-MMPR metabolite concentration is rather poor for clinical practice (AUC 0.65), which may be explained by the fact that hepatotoxicity also occurred in patients with lower 6-MMPR levels. Previous studies have reported similar results, indicating that thiopurine-associated hepatotoxicity is caused by one or more other determinants, such as the duration of exposure to high 6-MMPR levels or idiosyncratic reactions. ${ }^{8,10,15,21,22}$ 
Patients with hepatotoxicity often complain of various symptoms, including nausea, anorexia, fatigue, malaise and abdominal pain. ${ }^{11,22}$ In our study, signs of hepatotoxicity correlated well with gastrointestinal intolerance, but not with general malaise. Nausea and general malaise are the most frequently observed dose-dependent adverse reactions of thiopurines. ${ }^{23}$ Recently, it has been reported on a large long-term follow-up study that up to $80 \%$ of patients with thiopurine-induced nausea had to discontinue therapy. ${ }^{6}$ In the present study, patients who reported gastrointestinal complaints or general malaise were 2.5 to 3.5 times more likely to discontinue thiopurine treatment. Also, patients exceeding the T1 6-MMPR threshold were up to 2.5 times more at risk to experience gastrointestinal complaints or general malaise. Although this observation points to a dose-dependent or 6-MMPR concentration-dependent character, the discriminative value again is rather poor, suggesting that other causative factors may play an additional role. ${ }^{24,25}$

TPMT enzyme activity in the liver and erythrocytes are similar, therefore it is plausible to assume that high TPMT activity, presumably resulting in high erythrocyte 6-MMPR concentrations, may be a determining factor of thiopurine-induced hepatotoxicity, gastrointestinal complaints or general malaise. ${ }^{26}$ However, we did not observe a difference in TPMT enzyme activity in both patient groups, which suggests that there may be other mechanisms resulting in the extent of 6-MMPR production. ${ }^{27}$

The exact pathogenesis of thiopurine-induced hepatotoxicity is currently unknown. In vitro and animal studies point to involvement of depletion of the hepatoprotective antioxidant glutathione, increased oxidative stress, mitochondrial injury and proinflammatory factors, which may result in apoptotic or necrotic cell death. ${ }^{28-32}$ Xanthine oxidase (XO), involved in thiopurine metabolism, exerts the metabolic conversion of 6MP into 6-thiouric acid and has shown to be a critical source of free reactive oxygen species, potentially leading to toxic hepatocyte damage by oxidative stress. ${ }^{28,33}$ As the XO enzyme activity in the human liver is about $20 \%$ higher in males than females, it may potentially account for the male gender predominance of thiopurine-induced liver injury. $^{28,34,35}$

Except for the male gender and cytotoxic 6-MMPR metabolite concentrations, other specific risk factors for thiopurine-associated hepatotoxicity are currently unknown. ${ }^{8,10,12,35}$ It has been shown that concomitant corticosteroid therapy was associated with hepatotoxicity and a protective role of anti-TNF therapy has been suggested, findings we could not confirm in our study population. ${ }^{7}$ In general, it is known that adults are more susceptible for drug-induced hepatotoxicity than children. The most common susceptibility factors contributing to drug hepatotoxicity are age (>60 year) and genetic variability. ${ }^{36}$ In addition, obesity, alcohol and malnutrition may deplete glutathione in hepatocytes, which may increase susceptibility for drug-induced hepatotoxicity. Obviously, patients with hyperlipidemia frequently show liver test abnormalities because of non-alcoholic fatty liver disease, which may presumably be secondary to an older age, overweight and in some cases the (previous) use of corticosteroids. $^{36,37}$ In the present study, we found that thiopurine-induced 
hepatotoxicity independently correlated with a patient's gender, age, bodyweight and $\mathrm{BMI}$. Age and BMI demonstrated the best discriminative value to predict hepatotoxicity in patients starting thiopurine therapy. Thiopurine-induced hepatotoxicity therefore may be explained by influence of multiple factors, of which the 6-MMPR metabolites certainly seem a critical parameter. Therefore, based on these relevant determinants, we developed a multivariable regression model to predict hepatotoxicity with good accuracy (AUC 0.83). ${ }^{24}$ Males, irrespective of age, with a BMI $>25 \mathrm{~kg} / \mathrm{m}^{2}$ and 6-MMPR concentrations at week 1 exceeding the defined threshold of $3,615 \mathrm{pmol} / 8 \times 10^{8} \mathrm{RBC}$, were at highest risk. Females meeting these criteria were then at highly increased risk of hepatotoxicity, followed by males or females older than 50 years with a BMI $>25 \mathrm{~kg} / \mathrm{m}^{2}$ with $T 1$ 6-MMPR concentrations below the defined threshold. The proposed multivariable regression model may be used in clinical practice as a predictive algorithm to classify patients and determine the degree of risk for thiopurine-induced hepatotoxicity.

Several remarks should be made concerning the study design and observations. First, in the present prospective study we observed a hepatotoxicity incidence of $17 \%$, which is higher compared to previous reports on IBD patients. ${ }^{7,38}$ In these reports predefined dose escalation schedules were described, while our patients received the full thiopurine dose directly from start. The lack of a universally standardised definition of drug-induced liver toxicity and the absence of specific tests or markers may be another explanation. In previous retrospective and prospective studies different definitions of hepatotoxicity have been used, which make it hard to evaluate the exact prevalence or incidence in IBD patients using standard dosages of thiopurines. ${ }^{8}$ Elevations of $\gamma$-glutamyl transpeptidase and total or conjugated bilirubine were not evaluated in our study, since we aimed to focus specifically on hepatocellular and cholestatic hepatitis. Second, alcohol abuse, malnutrition, steatohepatitis, viral hepatitis infections and the use of other hepatotoxic drugs that may play a role in the development of liver injury were not evaluated. ${ }^{22,36}$ Third, gastrointestinal intolerance and hepatotoxicity were more frequently reported in patients treated with $6 \mathrm{MP}$, when compared to AZA. Although $\mathrm{AZA}$ and $6 \mathrm{MP}$ were equivalently dosed (AZA 2-2.5 mg/kg/day and 6-MP $1-1.5 \mathrm{mg} / \mathrm{kg} /$ day), this finding may partly be explained by the higher 6-MMPR concentrations found in patients treated with 6MP. In addition, 6MP dose was higher in patients who developed hepatotoxicity resulting from a statistically significant higher body weight, of which we demonstrated to be related to hepatotoxicity.

Therapeutic drug monitoring of thiopurine metabolites is currently advocated in case of therapy resistance to reveal non-adherence or underdosing, and in case of dosedependent adverse events. ${ }^{39}$ Early identification of patients at risk for thiopurine limiting adverse reactions is important in order to prevent unnecessary therapeutic failure. The findings of the present study indicate that already one week after initiation elevated 6-MMPR metabolite concentrations can be revealed, before steady-state levels have reached. ${ }^{18}$ In clinical practice, the proposed predictive algorithm may be used to identify patients at risk for thiopurine-induced hepatotoxicity. ${ }^{18}$ In these 
patients intensive liver test monitoring is warranted. For patients showing high 6-MMPR concentrations resulting from overdosing or a skewed metabolism, an optimisation strategy can be considered, including reducing the thiopurine dose or a switch to alternative therapy (e.g. 6-thioguanine or a dose reduction combined with allopurinol) in order to prevent 6-MMPR-associated liver toxicity, gastrointestinal intolerance or general malaise. ${ }^{40-45}$ Future studies should evaluate whether the predictive algorithm and the proposed preventive strategies result in a reduction of the incidence of these adverse reactions.

In conclusion, 6-MMPR metabolite measurement, one week after thiopurine initiation, is helpful to identify patients who are at increased risk of developing hepatotoxicity, gastrointestinal intolerance and general malaise, the most common limiting adverse events of thiopurines. A predictive algorithm was developed for thiopurine-induced hepatotoxicity, based on the 6-MMPR threshold parameter and the relevant determinants age, gender and BMI. 


\section{References}

1. Prefontaine E, Macdonald JK, Sutherland LR. Azathioprine or 6-mercaptopurine for induction of remission in Crohn's disease. Cochrane Database Syst Rev 2009(4):CD000545.

2. Prefontaine E, Sutherland LR, Macdonald JK, Cepoiu M. Azathioprine or 6-mercaptopurine for maintenance of remission in Crohn's disease. Cochrane Database Syst Rev 2009(1):CD000067.

3. Timmer A, McDonald JW, Macdonald JK. Azathioprine and 6-mercaptopurine for maintenance of remission in ulcerative colitis. Cochrane Database Syst Rev 2007(1):CD000478.

4. Derijks L, Wong DR. Pharmacogenetics of thiopurines in inflammatory bowel disease. Curr Pharm Des 2010;16:145-54.

5. Jharap B, Seinen ML, de Boer NK, van Ginkel JR, Linskens RK, Kneppelhout JC, Mulder CJ, van Bodegraven AA. Thiopurine therapy in inflammatory bowel disease patients: analyses of two 8-year intercept cohorts. Inflamm Bowel Dis 2010;16:1541-9.

6. Chaparro M, Ordás I, Cabré E, Garcia-Sanchez V, Bastida G, Peñalva M, Gomollón F, García-Planella E, Merino O, Gutiérrez A, Esteve M, Márquez L, Garcia-Sepulcre M, Hinojosa J, Vera I, Muñoz F, Mendoza JL, Cabriada JL, Montoro MA, Barreiro-de Acosta M, Ceña G, Saro C, Aldeguer X, Barrio J, Maté J, Gisbert JP. Safety of thiopurine therapy in inflammatory bowel disease: long-term follow-up study of 3931 patients. Inflamm Bowel Dis 2013;19:1404-10.

7. Bastida G, Nos P, Aguas M, Beltrán B, Rubín A, Dasí F, Ponce J. Incidence, risk factors and clinical course of thiopurine-induced liver injury in patients with inflammatory bowel disease. Aliment Pharmacol Ther 2005;22:775-82.

8. Gisbert JP, Gonzalez-Lama Y, Mate J. Thiopurine-induced liver injury in patients with inflammatory bowel disease: a systematic review. Am J Gastroenterol 2007;102:1518-27.

9. Bermejo F, López-Sanromán A, Algaba A, Van-Domselaar M, Gisbert JP, García-Garzón S, Garrido E, Piqueras B, De La Poza G, Guerra I. Mercaptopurine rescue after azathioprine-induced liver injury in inflammatory bowel disease. Aliment Pharmacol Ther 2010;31:120-4.

10. Dubinsky MC, Lamothe S, Yang HY, Targan SR, Sinnett D, Théorêt Y, Seidman EG. Pharmacogenomics and metabolite measurement for 6-mercaptopurine therapy in inflammatory bowel disease. Gastroenterology 2000;118:705-13.

11. Dubinsky MC, Yang H, Hassard PV, Seidman EG, Kam LY, Abreu MT, Targan SR, Vasiliauskas EA. 6-MP metabolite profiles provide a biochemical explanation for 6-MP resistance in patients with inflammatory bowel disease. Gastroenterology 2002;122:904-15.

12. Nygaard U, Toft N, Schmiegelow K. Methylated metabolites of 6-mercaptopurine are associated with hepatotoxicity. Clin Pharmacol Ther 2004;75:274-81.

13. Cuffari C, Theoret Y, Latour S, Seidman G. 6-Mercaptopurine metabolism in Crohn's disease: correlation with efficacy and toxicity. Gut 1996;39:401-6.

14. Goldenberg BA, Rawsthorne $P$, Bernstein CN. The utility of 6-thioguanine metabolite levels in managing patients with inflammatory bowel disease. Am J Gastroenterol 2004;99:1744-8.

15. Wright S, Sanders DS, Lobo AJ, Lennard L. Clinical significance of azathioprine active metabolite concentrations in inflammatory bowel disease. Gut 2004;53:1123-8.

16. Coenen MJ, de Jong DJ, van Marrewijk CJ, Derijks LJ, Vermeulen SH, Wong DR, Klungel OH, Verbeek ALM, Hooymans PM, Peters WHM, te Morsche RHM, Newman WG, Scheffer H, Guchelaar H-J, Franke B. Screening of the TPMT gene followed by personalized dosing of azathioprine and mercaptopurine treatment reduces the risk of leucopenia in patients with inflammatory bowel disease: a randomized trial. Submitted for publication 2014.

17. Swen JJ, Nijenhuis M, de Boer A, Grandia L, Maitland-van der Zee AH, Mulder H, Rongen GA, van Schaik RH, Schalekamp T, Touw DJ, van der Weide J, Wilffert B, Deneer VH, Guchelaar HJ. Pharmacogenetics: from bench to byte--an update of guidelines. Clin Pharmacol Ther 2011;89:662-73.

18. Derijks LJ, Gilissen LP, Engels LG, Bos LP, Bus PJ, Lohman JJ, Curvers WL, Van Deventer SJ, Hommes DW, Hooymans PM. Pharmacokinetics of 6-mercaptopurine in patients with inflammatory bowel disease: implications for therapy. Ther Drug Monit 2004;26:311-8.

19. Ford L, Graham V, Berg J. Whole-blood thiopurine S-methyltransferase activity with genotype concordance: a new, simplified phenotyping assay. Ann Clin Biochem 2006;43:354-60. 
20. Ford LT, Berg JD. Determination of thiopurine S-methyltransferase activity in erythrocytes using 6thioguanine as substrate and a non-extraction liquid chromatographic technique. J Chromatogr $B$ Analyt Technol Biomed Life Sci 2003 5;798:111-5.

21. Shaye OA, Yadegari M, Abreu MT, Poordad F, Simon K, Martin P, Papadakis KA, Ippoliti A, Vasiliauskas E, Tran TT. Hepatotoxicity of 6-mercaptopurine (6-MP) and Azathioprine (AZA) in adult IBD patients. Am J Gastroenterol 2007;102:2488-94.

22. Navarro VJ, Senior JR. Drug-related hepatotoxicity. N Engl J Med 2006;354:731-9.

23. de Jong DJ, Derijks LJ, Naber AH, Hooymans PM, Mulder CJ. Safety of thiopurines in the treatment of inflammatory bowel disease. Scand J Gastroenterol Suppl 2003(239):69-72.

24. Fan J, Upadhye S, Worster A. Understanding receiver operating characteristic (ROC) curves. CJEM 2006;8:19-20.

25. McGovern DP, Travis SP, Duley J, Shobowale-Bakre el M, Dalton HR. Azathioprine intolerance in patients with IBD may be imidazole-related and is independent of TPMT activity. Gastroenterology 2002;122:838-9.

26. Szumlanski CL, Honchel R, Scott MC, Weinshilboum RM. Human liver thiopurine methyltransferase pharmacogenetics: biochemical properties, liver-erythrocyte correlation and presence of isozymes. Pharmacogenetics 1992;2:148-59.

27. van Egmond R, Chin P, Zhang M, Sies CW, Barclay ML. High TPMT enzyme activity does not explain drug resistance due to preferential 6-methylmercaptopurine production in patients on thiopurine treatment. Aliment Pharmacol Ther 2012;35:1181-9.

28. Tapner MJ, Jones BE, Wu WM, Farrell GC. Toxicity of low dose azathioprine and 6-mercaptopurine in rat hepatocytes. Roles of xanthine oxidase and mitochondrial injury. J Hepatol 2004;40:454-63.

29. Menor C, Fernández-Moreno MD, Fueyo JA, Escribano O, Olleros T, Arriaza E, Cara C, Lorusso M, Di Paola M, Román ID, Guijarro LG. Azathioprine acts upon rat hepatocyte mitochondria and stressactivated protein kinases leading to necrosis: protective role of $\mathrm{N}$-acetyl-L-cysteine. J Pharmacol Exp Ther 2004;311:668-76.

30. Eklund BI, Moberg M, Bergquist J, Mannervik B. Divergent activities of human glutathione transferases in the bioactivation of azathioprine. Mol Pharmacol 2006;70:747-54.

31. Lee AU, Farrell GC. Mechanism of azathioprine-induced injury to hepatocytes: roles of glutathione depletion and mitochondrial injury. J Hepatol 2001;35:756-64.

32. Russmann S, Kullak-Ublick GA, Grattagliano I. Current concepts of mechanisms in drug-induced hepatotoxicity. Curr Med Chem 2009;16:3041-53.

33. Matsuo K, Sasaki E, Higuchi S, Takai S, Tsuneyama K, Fukami T, Nakajima M, Yokoi T. Involvement of oxidative stress and immune- and inflammation-related factors in azathioprine-induced liver injury. Toxicol Lett 2014;224:215-24.

34. Guerciolini R, Szumlanski C, Weinshilboum RM. Human liver xanthine oxidase: nature and extent of individual variation. Clin Pharmacol Ther 1991;50:663-72.

35. Present DH, Meltzer SJ, Krumholz MP, Wolke A, Korelitz BI. 6-Mercaptopurine in the management of inflammatory bowel disease: short- and long-term toxicity. Ann Intern Med 1989;111:641-9.

36. Larrey D. Drug-induced liver diseases. J Hepatol 2000;32(1 Suppl):77-88.

37. Rojas-Feria M, Castro M, Suarez E, Ampuero J, Romero-Gomez M. Hepatobiliary manifestations in inflammatory bowel disease: the gut, the drugs and the liver. World J Gastroenterol 2013;19:7327-40.

38. Hindorf $U$, Lindqvist $M$, Peterson $C$, Söderkvist $P$, Ström $M$, Hjortswang $H$, Pousette A, Almer S. Pharmacogenetics during standardised initiation of thiopurine treatment in inflammatory bowel disease. Gut 2006;55:1423-31.

39. van Asseldonk DP, Sanderson J, de Boer NK, Sparrow MP, Lémann M, Ansari A, Almer SH, Florin TH, Gearry RB, Mulder CJ, Mantzaris G, van Bodegraven AA; Thiopurine Task Force Interest Group. Difficulties and possibilities with thiopurine therapy in inflammatory bowel disease--proceedings of the first Thiopurine Task Force meeting. Dig Liver Dis 2011;43:270-6.

40. de Boer NK, Jharap B, Mulder C, van Bodegraven AA. Low and adequately dosed 6-thioguanine: not so bad after all. Inflamm Bowel Dis 2008;14:1166-7.

41. Gilissen LP, Derijks L, Driessen A, Bos LP, Hooymans PM, Stockbrügger RW, Engels LG. Toxicity of 6-thioguanine: no hepatotoxicity in a series of IBD patients treated with long-term, low dose 6-thioguanine. Some evidence for dose or metabolite level dependent effects? Dig Liver Dis 2007;39: 156-9. 
42. van Asseldonk DP, Seinen ML, de Boer NK, van Bodegraven AA, Mulder CJ. Hepatotoxicity associated with 6-methyl mercaptopurine formation during azathioprine and 6-mercaptopurine therapy does not occur on the short-term during 6-thioguanine therapy in IBD treatment. J Crohns Colitis 2012;6:95-101.

43. Ansari A, Elliott T, Baburajan B, Mayhead P, O'Donohue J, Chocair P, Sanderson J, Duley J. Long-term outcome of using allopurinol co-therapy as a strategy for overcoming thiopurine hepatotoxicity in treating inflammatory bowel disease. Aliment Pharmacol Ther 2008;28:734-41.

44. Leong RW, Gearry RB, Sparrow MP. Thiopurine hepatotoxicity in inflammatory bowel disease: the role for adding allopurinol. Expert Opin Drug Saf 2008;7:607-16.

45. Hoentjen F, Seinen ML, Hanauer SB, de Boer NK, Rubin DT, Bouma G, Harrell LE, van Bodegraven AA. Safety and effectiveness of long-term allopurinol-thiopurine maintenance treatment in inflammatory bowel disease. Inflamm Bowel Dis 2013;19:363-9. 


\section{Chapter 8}

Safe 6-thioguanine therapy of a TPMT deficient Crohn's disease patient by using therapeutic drug monitoring

WGN Mares, DR Wong, LPL Gilissen, AAM Masclee, PM Hooymans, LGJB Engels J Crohns Colitis 2009;3:128-130 


\section{Abstract}

The immunosuppressive thiopurines, azathioprine (AZA) and 6-mercaptopurine (6-MP), have proven efficacy in steroid-dependent or refractory inflammatory bowel disease (IBD). In case of TPMT deficiency serious myelosuppression may occur. 6-thioguanine (6-TG) has been suggested in case of AZA and 6-MP resistant or intolerant patients.

Our case demonstrates that very low dose 6-TG under close clinical surveillance and frequent therapeutic drug monitoring, may be a rescue drug for IBD-patients with low or without functional TPMT activity. 


\section{Introduction}

In steroid-dependent or refractory inflammatory bowel diseases (IBD) the thiopurines, azathioprine (AZA) and 6-mercaptopurine (6-MP) are effective, but also harbour potentially serious side effects. ${ }^{1,2}$ Main thiopurine metabolites are the active 6-thioguanine nucleotides (6-TGN) and 6-methylmercaptopurine ribonucleotides (6-MMPR).

Thiopurine S-methyltransferase (TPMT) is considered as the most important enzyme in this regard. ${ }^{3}$ Because of its genetic polymorphisms serious myelosuppression may arise on standard AZA/6-MP therapy in case of TPMT deficiency. ${ }^{4}$ Recently another thiopurine compound, 6-thioguanine (6-TG), has been suggested as an alternative in case of AZA and 6-MP resistance or failure. ${ }^{5,6}$

We describe the case of a 20-year-old man with fistulising Crohn's disease, who developed severe pancytopenia on a normal dose 6-MP due to complete TPMT deficiency. The patient was successfully treated with a very low dose of 6-TG in combination with infliximab.

\section{Case}

In 1992 a 20-year-old man with ileocolonic Crohn's disease (CD) was successfully treated with sulphasalazine and prednisolone for one year. The patient withdrew from out patient clinic control against medical advice. In 1999 urgent subtotal colectomy had to be performed because of toxic perforated colitis. Subsequently, he was treated with oral mesalazine. Furthermore in $\mathbf{2 0 0 2}$ he was operated for parastomal herniation using mash grafts. In 2004, a fistula tract appeared adjacent to the ileostoma trespassing the mash grafts. Also active $C D$ in the neoterminal ileum including the ileostomy was found. $6-\mathrm{MP}$ was started at $50 \mathrm{mg}$ daily $(0.64 \mathrm{mg} / \mathrm{kg}$ body weight). Four weeks later the patient was admitted for severe myelosuppression (haemoglobin $4.3 \mathrm{mmol} / \mathrm{l}$, thrombocytes $24 \times 10^{9} / \mathrm{l}$, leukocytes $\left.0.9 \times 10^{9} / \mathrm{l}\right)$. The $6-\mathrm{TGN}$ concentration was myelotoxic $(1,282 \mathrm{pmol} /$ $8 \times 10^{8}$ red blood cells (RBC) and 6-MMPR-level was negative.

Phenotyping revealed non-detectable TPMT-activity. 6-MP was discontinued and the patient was treated with erythrocyte and thrombocyte transfusions. Two months later blood constituents were completely normalised. Because of ileocutaneous fistulae he was treated with repeated infliximab infusions ( $5 \mathrm{mg} / \mathrm{kg}$ body weight) at week 0,2 and 6 and then every 8 weeks with temporary improvement. Fistula output decreased after infliximab infusion, but started to produce some weeks before the next infusion.

Because of this condition, and regarding the TPMT deficiency, in 2006 a very low dose of 6-TG (20 mg per week, $0.036 \mathrm{mg} / \mathrm{kg}$ body weight daily) was started after obtaining informed consent about possible side-effects. 6-TGN levels, biochemical safety parameters and complete blood counts were obtained every week. After 1, 2 and 3 weeks the 6 -TGN trough levels were 460,674 and $1,003 \mathrm{pmol} / 8 \times 10^{8} \mathrm{RBC}$ respectively, 
without developing myelosuppression. Since 6-TGN trough levels raised till $1,003 \mathrm{pmol} / 8 \times 10^{8} \mathrm{RBC}$, the 6-TG dose was adjusted to $20 \mathrm{mg}$ every two weeks $(0.018 \mathrm{mg} / \mathrm{kg}$ body weight daily) resulting in 6-TGN trough levels between 500 and $900 \mathrm{pmol} / 8 \times 10^{8} \mathrm{RBC}$.

After starting 6-TG in combination with infliximab the fistulae closed and the patient was in clinical remission. Complete blood counts and liver tests remained normal, for 30 months now. The patient was advised to undergo a liver biopsy to exclude nodular regenerative hyperplasia after twelve months of treatment, but he refused.

\section{Discussion}

In IBD patients on AZA or 6-MP treatment, both 6-TGN and 6-MMPR concentrations do not correlate with drug dose per kilograms bodyweight and show large interindividual differences. Therapeutic drug monitoring (TDM) may help improving the efficacy by demonstrating suboptimal dosing and predicting risks of complications such as leukopenia and hepatitis. ${ }^{7,8}$ Generally 6 -TGN levels greater than $235 \mathrm{pmol} / 8 \times 10^{8} \mathrm{RBC}$ are recommended as a therapeutic cut-off level for patients on AZA or 6-MP., ${ }^{3,9}$ High levels of 6-TGN may cause myelotoxicity; levels above $500 \mathrm{pmol} / 8 \times 10^{8} \mathrm{RBC}$ increase the risk for leukocytopenia or even pancytopenia, whereas 6-MMPR-levels above $5,700 \mathrm{pmol} / 8 \times 10^{8} \mathrm{RBC}$ are correlated with hepatotoxicity. ${ }^{3,7}$

IBD patients with homozygous mutant and hence severe TPMT deficiency are generally recommended not to receive AZA or 6-MP at all. ${ }^{5}$ In Caucasian population the frequency distribution of TPMT activity is trimodal: approximately $89 \%$ of the population are homozygous for the wild-type allele $\left(T P M T^{H}\right)$ with high enzyme activity, $11 \%$ are heterozygous with intermediate TPMT activity $\left(T P M T^{H} / T P M T^{L}\right)$ and 1 in 300 subjects have low or no functional activity $\left(T P M T^{L} / T P M T^{L}\right) .{ }^{10}$

In case of TPMT deficiency thiopurine therapy is dangerous, because of the increased risk of development of life-threatening pancytopenia. ${ }^{5}$ Nevertheless, two cases have been described demonstrating safe thiopurine therapy in homozygous TPMT mutancy by applying very low drug dose (AZA $0.16-0.29 \mathrm{mg} / \mathrm{kg} /$ day; normally a drug dose of $2-2.5 \mathrm{mg} / \mathrm{kg} /$ day is used in IBD-patients) and therapeutic drug monitoring. ${ }^{11}$

In the presented patient, pancytopenia developed after starting 6-MP therapy with high 6-TGN levels (up to $1,282 \mathrm{pmol} / 8 \times 10^{8} \mathrm{RBC}$ ), resulting from a complete TPMTdeficiency. After discontinuation of 6-MP and starting infliximab monotherapy, Crohn's disease was not in remission. The patient refused to receive 6-MP anymore. Adalimumab was not available in the Netherlands at the time. Surgery was discussed with the patient, but considered to be a suboptimal option because of the earlier operations and the implicated large resection. The patient refused surgery for this reasons and chose for a conservative treatment. 
6-TG seems a good alternative for patients who have to discontinue AZA or 6-MP because of side-effects or lack of clinical response. Several studies proved good shortterm safety and efficacy. ${ }^{6,12-14}$ In contrast with the metabolism of AZA and 6-MP, 6-TG is more directly converted to the active 6-TGN metabolites, without the formation of hepatotoxic 6-MMPR. ${ }^{14}$ No dose-finding studies have been performed, but previous reports showed good efficacy with relatively low 6-TG doses (not exceeding $20 \mathrm{mg} /$ day)..$^{12}$

Because 6-TG is partly methylated by TPMT to 6-methylthioguanine (6-MTG), 6-TG was prescribed in a very low dose in our case, starting with $20 \mathrm{mg}$ every week $(0.036 \mathrm{mg} / \mathrm{kg} /$ day $)$ using therapeutic drug monitoring, complete blood count and biochemical parameters measurement weekly.

For 6-TG therapy in IBD patients, no clear therapeutic or toxic 6-TGN levels have been described yet. It seems however that hepatotoxicity, in particular nodular regenerative hyperplasia (NRH) and veno-occlusive disease (VOD), is dose-dependent and related with 6-TGN levels higher than $1,000 \mathrm{pmol} / 8 \times 10^{8} \mathrm{RBC}^{12,15,16}$ In our case, the 6 -TG dose was adjusted to $20 \mathrm{mg}$ every other week $(0.018 \mathrm{mg} / \mathrm{kg} /$ day) and 6-TGN trough levels were between 500 and $900 \mathrm{pmol} / 8 \times 10^{8} \mathrm{RBC}$. The patient has been treated successfully with infliximab and 6-TG and the CD has already been in remission for 30 months.

6-TG seems a safe and effective alternative thiopurine in AZA- and 6-MP intolerant IBD patients, when used in a low dose and under strict control. Our case demonstrates that 6-TG in a very low dose may be an effective and safe rescue drug for IBD-patients without functional activity of TPMT who develop myelosuppression on AZA or 6-MP therapy and who are refractory or intolerant for all other immunosuppressives. Therapeutic drug monitoring is crucial in partial or complete TPMT deficiency and this therapy should be given under close surveillance of 6-TGN levels, complete blood counts and biochemical parameters to detect myelosuppression and hepatotoxicity. Therapeutic 6-TGN levels when using 6-TG are not exactly known. A safe and therapeutic level is probably between 500 and 800 pmol $/ 8 \times 10^{8} \mathrm{RBC}^{16}$ Before starting 6-TG therapy the patient should be informed about the possible risks of 6-TG. We suggest that 6-TGN levels are measured weekly after starting 6-TG in IBD-patients with diminished or without functional TPMT activity. The 6-TG dose should be adjusted by means of 6-TGN level measurement until therapeutic steady-state 6-TGN levels are reached. 


\section{References}

1. Pearson DC, May GR, Fick GH, Sutherland LR. Azathioprine and 6-mercaptopurine in Crohn disease. A meta-analysis. Ann Intern Med 1995;123:132-42.

2. Adler DJ, Korelitz BI. The therapeutic efficacy of 6-mercaptopurine in refractory ulcerative colitis. Am J Gastroenterol 1990;85:717-22.

3. Dubinsky MC, Lamothe S, Yang HY, Targan SR, Sinnett D, Théorêt Y, Seidman EG. Pharmacogenomics and metabolite measurement for 6-mercaptopurine therapy in inflammatory bowel disease. Gastroenterology 2000;118:705-13.

4. Lennard L, Van Loon JA, Weinshilboum RM. Pharmacogenetics of acute azathioprine toxicity: relationship to thiopurine methyltransferase genetic polymorphism. Clin Pharmacol Ther 1989;46: 149-54.

5. Sandborn WJ. Rational dosing of azathioprine and 6-mercaptopurine. Gut 2001;48:591-2.

6. Dubinsky MC, Hassard PV, Seidman EG, Kam LY, Abreu MT, Targan SR, Vasiliauskas EA. An open-label pilot study using thioguanine as a therapeutic alternative in Crohn's disease patients resistant to 6mercaptopurine therapy. Inflamm Bowel Dis 2001;7:181-9.

7. Derijks LJ, Gilissen LP, Engels LG, Bos LP, Bus PJ, Lohman JJ, Curvers WL, Van Deventer SJ, Hommes DW, Hooymans PM. Pharmacokinetics of 6-mercaptopurine in patients with inflammatory bowel disease: implications for therapy. Ther Drug Monit 2004;26:311-8.

8. Gilissen LP, Derijks L, Bos LP, Bus PJ, Hooymans PM, Engels LG. Therapeutic drug monitoring in patients with inflammatory bowel disease and established azathioprine therapy. Clin Drug Investig 2004;24: 479-86.

9. Cuffari C, Hunt S, Bayless T. Utilisation of erythrocyte 6-thioguanine metabolite levels to optimise azathioprine therapy in patients with inflammatory bowel disease. Gut 2001;48:642-6.

10. Lennard L. TPMT in the treatment of Crohn's disease with azathioprine. Gut 2002;51:143-6.

11. Kaskas BA, Louis E, Hindorf U, Schaeffeler E, Deflandre J, Graepler F, Schmiegelow K, Gregor M, Zanger UM, Eichelbaum M, Schwab M. Safe treatment of thiopurine S-methyltransferase deficient Crohn's disease patients with azathioprine. Gut 2003;52:140-2.

12. Derijks L, de Jong DJ, Gilissen LP, Engels LG, Hooymans PM, Jansen JB, Mulder CJ. 6-Thioguanine seems promising in azathioprine- or 6-mercaptopurine-intolerant inflammatory bowel disease patients: a short-term safety assessment. Eur J Gastroenterol Hepatol 2003;15:63-7.

13. Herrlinger KR, Kreisel W, Schwab $M$, Schoelmerich J, Fleig WE, Ruhl A, Reinshagen $M$, Deibert $P$, Fellermann K, Greinwald R, Stange EF. 6-thioguanine--efficacy and safety in chronic active Crohn's disease. Aliment Pharmacol Ther 2003;17:503-8.

14. Dubinsky MC, Feldman EJ, Abreu MT, Targan SR, Vasiliauskas EA. Thioguanine: a potential alternate thiopurine for IBD patients allergic to 6-mercaptopurine or azathioprine. Am J Gastroenterol 2003;98:1058-63.

15. de Boer NK, Derijks L, Gilissen LP, Hommes DW, Engels LG, de-Boer SY, den Hartog G, Hooymans PM, Mäkelburg $A B$, Westerveld $B D$, Naber $A H$, Mulder $C J$, de Jong DJ. On tolerability and safety of a maintenance treatment with 6-thioguanine in azathioprine or 6-mercaptopurine intolerant IBD patients. World J Gastroenterol 2005;11:5540-4.

16. Gilissen LP, Derijks LJ, Driessen A, Bos LP, Hooymans PM, Stockbrügger RW, Engels LG. Toxicity of 6-thioguanine: no hepatotoxicity in a series of IBD patients treated with long-term, low dose 6-thioguanine. Some evidence for dose or metabolite level dependent effects? Dig Liver Dis 2007;39:156-9. 


\section{Part III}

Pharmacokinetic interactions and thiopurines 



\section{Chapter 9}

Dose-dependent influence of 5-aminosalicylates on thiopurine metabolism

NKH de Boer, DR Wong, B Jharap, P de Graaf, PM Hooymans, CJJ Mulder, F Rijmen, LGJB Engels, AA van Bodegraven

Am J Gastroenterol 2007;102:2747-2753 


\section{Abstract}

Introduction

Studies indicated that 5-aminosalicylates (5-ASA) may influence the metabolism of thiopurines; however, conclusions were restricted as a result of number of patients or study design.

Aim

To determine the influence of 5-ASA on thiopurine metabolism, we performed a prospective multicenter pharmacokinetic interaction study of two different 5-ASA dosages ( $2 \mathrm{~g}$ daily followed by $4 \mathrm{~g}$ daily) in 26 IBD patients during steady-state AZA or 6-MP therapy.

Results

The 4-weeks coadministration of $2 \mathrm{~g}$ 5-ASA daily, followed by a 4-weeks period of $4 \mathrm{~g}$ 5-ASA daily, led to a statistical significant increase of $40 \%$ (absolute $84 \mathrm{pmol} / 8 \times 10^{8} \mathrm{RBC}$ ) and $70 \%$ (absolute $154 \mathrm{pmol} / 8 \times 10^{8} \mathrm{RBC}$ ) in 6-thioguanine nucleotide levels (6-TGN), respectively. A rise in 6-TGN levels was observed in $100 \%$ of patients after a 4 -weeks period of $4 \mathrm{~g}$ 5-ASA daily. The 6-methylmercaptopurine ribonucleotides levels did not change. Signs of myelotoxicity were observed in $7.7 \%$ of patients $(n=2)$.

Conclusions

The level of the pharmacological active 6-TGN significantly increases in a dose-dependent manner during 5-ASA coadministration. IBD patients who are unresponsive or refractory to standard thiopurine therapy may benefit from the coadministration of 5-ASA, leading to an increase in 6-TGN levels. 


\section{Introduction}

Azathioprine (AZA) and 6-mercaptopurine (6-MP) are frequently used immunosuppressive drugs in the treatment of ulcerative colitis (UC), indeterminate colitis (IC) and Crohn's disease (CD). ${ }^{1}$ The complex metabolism of these thiopurines has been partly unraveled in recent years (Figure 9.1). The 6-thioguanine nucleotides (6-TGN), consisting of three phosphorylated forms (6-thioguanine monophosphate, 6-thioguanine diphosphate and 6-thioguanine triphosphate), are considered to be the major pharmacologically active end-metabolites. ${ }^{2}$ Monitoring of 6-TGN in red blood cells (RBC) has been proposed to optimise thiopurine therapy. 6-Thioguanine nucleotides levels above $235 \mathrm{pmol} / 8 \times 10^{8} \mathrm{RBC}$ have been associated with the best probability of response, but grossly elevated 6-TGN levels (above $490 \mathrm{pmol} / 8 \times 10^{8} \mathrm{RBC}$ ) have been associated with an increased risk of developing myelotoxicity. ${ }^{3}$ The enzyme thiopurine S-methyltransferase (TPMT) plays a key role in the complex metabolisation process of thiopurines (Figure 9.1). High activity of TPMT will lead to an increased formation of methylated thiopurine metabolites (6-methylmercaptopurine (6-MMP) and 6-methylmercaptopurine ribonucleotides (6-MMPR)). High concentrations of 6-MMPR (above 5,700 pmol/ $8 \times 10^{8} \mathrm{RBC}$ ) during thiopurine therapy have been associated with an increased risk of developing hepatotoxicity, but also with treatment failure. ${ }^{3}$ A 6-MMPR/6-TGN ratio below 11 was found to correlate with clinical response to 6-MP therapy. Above this point a lack of efficacy is seen, even after dose escalation. ${ }^{4}$ Therefore, a substantial number of patients are unable to benefit from thiopurine therapy due to the development of inadequate metabolite levels.

Mesalazine (5-aminosalicylates (5-ASA)) is also a commonly prescribed drug in the treatment of inflammatory bowel diseases (IBD). The precise mechanism of action of 5-ASA is not known, but is likely due to a local anti-inflammatory effect from the luminal site in the diseased parts of the gut. ${ }^{5}$ The oral ingestion of uncoated mesalazine is followed by rapid and almost complete absorption in the upper intestine; absorption from the colon is considerably lower. Absorption of 5-ASA is followed by extensive metabolism to the major inactive $\mathrm{N}$-acetyl-5-aminosalicylate ( $\mathrm{N}$-acetyl-5-ASA) by the $\mathrm{N}$-acetyl-transferase 1 enzyme in intestinal epithelial cells and the liver.

Several studies indicated that 5-ASA may influence the metabolism of thiopurines, ${ }^{6-10}$ potentially leading to an increased risk of developing leukopenia due to the generation of elevated 6-TGN levels. ${ }^{11,12}$ However, conclusions were restricted due to number of patients or study design (e.g. withdrawal of 5-ASA compounds during maintenance combination therapy with 5 -ASA and thiopurines ${ }^{7,10}$ ). The mechanism of this interaction remains to be elucidated, although several authors have suggested that the TPMT activity is inhibited by sulphasalazine and other 5-ASA delivering drugs. ${ }^{6-8}$

In order to determine the influence of 5-ASA on thiopurine metabolism, we performed a prospective multicenter pharmacokinetic evaluation of two different 5-ASA dosages ( $2 \mathrm{~g}$ daily followed by $4 \mathrm{~g}$ daily) in $\mathrm{CD}$ and $\mathrm{UC}$ patients during steady maintenance AZA or 6-MP therapy. 


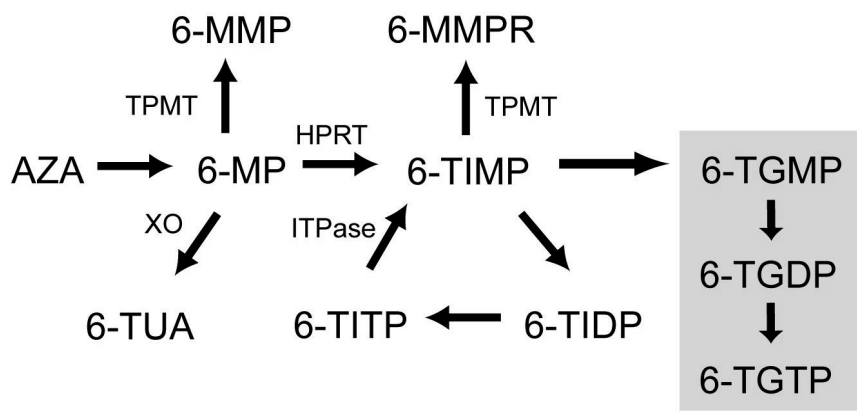

Figure 9.1 Thiopurine metabolism (simplified).

Azathioprine (AZA) is non-enzymatically degraded to 6-mercaptopurine (6-MP). Xanthine oxidase inactivates 6-MP by the formation of 6-thiouric acid (6-TUA). Thiopurine S-methyltransferase (TPMT) methylates 6-MP into 6-methylmercaptopurine (6-MMP). Via hypoxanthine phosphoribosyl transferase (HPRT), 6-MP is converted to 6-thioinosine monophosphate (6-TIMP). Via two other enzymatic steps, inosine monophosphate dehydrogenase (IMPDH) and guanosine monophosphate synthetase (GMPS), the pool of 6-thioguanine nucleotides (6-TGN) is ultimately generated, consisting of 6-thioguaninemonophosphate (6-TGMP), 6-thioguanine diphosphate (6-TGDP) and 6-thioguanine triphosphate (6-TGTP). 6-TIMP may also be methylated by TPMT leading to 6-methylmercaptopurine ribonucleotides (6-MMPR) (consisting of 6-methyl-thioinosine monophosphate, 6-methyl-thioinosine diphosphate and 6-methyl-thioinosine triphosphate). In a cycle, 6-TIMP may be phosphorylated to 6-thioinosine diphosphate (6-TIDP), subsequently to 6-thioinosin -triphosphate (6-TITP) and ultimately back to 6-TIMP due to inosine triphosphate pyrophosphatase (ITPase).

\section{Materials and methods}

\section{Patient selection}

Patients visiting the Outpatient Clinic of the VU University Medical Centre (university hospital, Amsterdam, the Netherlands) or the Maasland Hospital (general district hospital, Sittard, the Netherlands), were eligible for the study when meeting the following study criteria. Inclusion criteria were: age between 18 and $80 \mathrm{yr}$, diagnosis of UC, IC or CD for at least 6 months, immunosuppressive therapy with AZA or 6-MP in a stable dosage for at least eight consecutive weeks, normal kidney and liver tests (defined as aspartate transaminase (ASAT), alkaline phosphatase (AP) and/or creatinin levels below twice the upper reference limits). Exclusion criteria were: signs of myelosuppression (defined as a leukocyte count below $2.5 \times 10^{9} / \mathrm{l}$ and/or a platelet count below $100 \times 10^{9} / \mathrm{l}$ ), presence of an active infection (defined as fever in combination with a C-reactive protein (CRP) level above the upper reference limit), anaemia (defined as a haemoglobin $(\mathrm{Hb})$ level below $6 \mathrm{mmol} / \mathrm{l}$ ), known extensive proximal small bowel $C D$ possibly interfering with resorptive area, small bowel surgery 
significantly reducing the intestinal resorptive area, documented intolerance to 5-ASA compounds, use of 5-ASA compounds within the last 30 days before study entry, concomitant use of allopurinol, mycophenolate mofetil, angiotensin I-converting enzyme inhibitors, or diuretics, current pregnancy or intention to become pregnant within 6 months or lactation.

\section{Study design}

A prospective multicenter pharmacokinetic evaluation of the influence of two different 5-ASA dosages (Pentasa ${ }^{\circledR}$ microgranules, Ferring, Hoofddorp, the Netherlands) on thiopurine metabolism was carried out. To study the influence of 5-ASA and N-acetyl5-ASA on thiopurine metabolite levels, the prolonged-release ethylcellulose-coated mesalazine preparation (Pentasa ${ }^{\circledR}$ microgranules) were used. 5-ASA is released gradually from Pentasa ${ }^{\circledR}$ granules in the small and large intestine, nearly resulting in constant plasma concentrations of 5-ASA and N-acetyl-5-ASA. ${ }^{5}$. Demographic data and disease activity (Harvey-Bradshaw index for CD and Truelove-Witts index for UC) were collected at baseline. Patients received consecutively per protocol two different dosages of 5-ASA (Figure 9.2). Laboratory parameters $(\mathrm{Hb}$, leukocyte, platelet, erythrocyte count, mean cellular volume (MCV), CRP, ASAT, alanine transaminase (ALAT), AP, gammaglutamyl transferase (GGT), bilirubin, lactate dehydrogenase (LDH), albumin, amylase, and creatinin), 6-TGN levels, 6-MMPR levels, 5-ASA levels, and $\mathrm{N}$-acetyl-5-ASA levels were determined before initiation of 5-ASA therapy ( $T=1)$, after 4 weeks of 2 grams 5-ASA per day ( $T=2)$, after 4 weeks of 4 grams 5-ASA per day ( $T=3)$ and after 4 weeks following cessation of 5-ASA therapy $(T=4)$ (Figure 9.2). We chose a dosage regime of four weeks in the study design, as after approximately 20 days a steady-state concentration is expected.

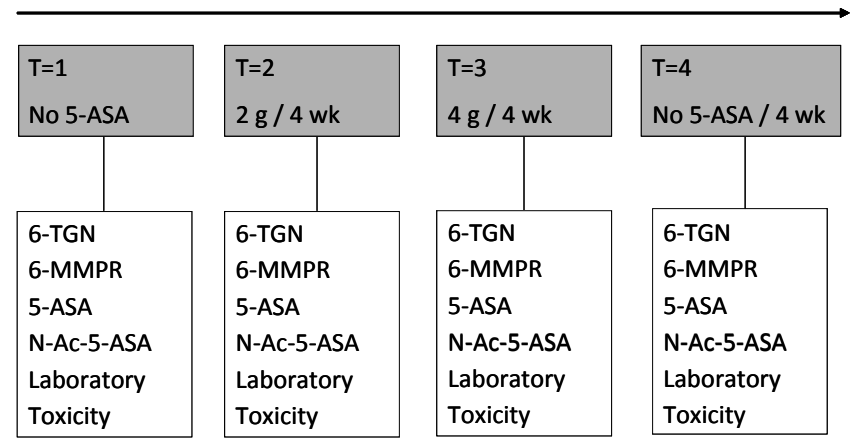

Figure 9.2 Trial design.

6-thioguanine nucleotides (6-TGN), 6-methymercaptopurine ribonucleotides (6-MMPR), 5-aminosalicylate (5-ASA), N-acetyl-5-aminosalicylic acid (N-Ac-5-ASA). 
The study protocol was approved by the ethical committees of the VU University Medical Centre and Maasland Hospital. All patients gave written consent before being enrolled in the study.

\section{Outcome measurements}

Primary outcome measures were the determination of the concentration of 6-TGN, 6-MMPR, 5-ASA and N-acetyl-5-ASA during the different 5-ASA regimes. Additionally, routine laboratory parameters were monitored for safety issues.

\section{Measurement of 6-TGN and 6-MMPR in erythrocytes}

The blood samples were collected in coated lithium-heparin tubes. The samples were centrifuged to isolate erythrocytes and after washing with PBS buffer solution, erythrocyte counts were performed. Samples were stored at $-20^{\circ} \mathrm{C}$ until analysis. Red blood cell 6-TGN and 6-MMPR levels were measured in the laboratory of the Department of Clinical Pharmacy \& Toxicology, Maasland Hospital (Sittard, The Netherlands) using a high performance liquid chromatography (HPLC) assay as previously reported. ${ }^{13}$

\section{Measurement of 5-ASA and N-acetyl-5-ASA levels}

Blood samples were collected in serum tubes. The samples were centrifuged for at least 5 minutes at $1500 \mathrm{x}$. Subsequently, the serum was separated and stored at $-20^{\circ} \mathrm{C}$ until analysis. 5-ASA and $\mathrm{N}$-acetyl-5-ASA serum levels were measured in the laboratory of the Department of Clinical Pharmacy \& Toxicology, Maasland Hospital (Sittard, The Netherlands) using a slightly modified assay. ${ }^{14}$ The within-run coefficient of variation was maximally $3.3 \%$ for both 5-ASA and $\mathrm{N}$-acetyl-5-ASA in the range of the calibration. The lower limit of quantification of the assay was determined at $10 \mathrm{ng} / \mathrm{ml}$ for both 5-ASA and N-acetyl-5-ASA.

\section{Statistical analysis}

Data are given descriptively and expressed as mean with standard deviation (SD) or median with range, when indicated. A mixed model analysis was used to test for differences in thiopurine metabolite levels across the four measurement occasions and for the determination of the influence of 5-ASA parameters. Paired sample $T$-tests were used to analyse the increase or decrease in 6-MMPR/6-TGN ratio. Correlations between parameters were determined using the Pearson's or Spearman's test, when indicated. $P$ values of less than 0.05 were considered significant. SPSS for windows version 11.0 (SPSS Inc., Chicago, IL) was used for statistical analysis. 


\section{Results}

\section{Patient demographics}

Twenty-nine IBD patients were screened and enrolled (twenty-five patients were enrolled by VU University Medical Centre and four patients by Maasland Hospital). Three patients were excluded from analysis: two patients due to protocol violation and one patient developed adverse events even before the initiation of 5-ASA coadministration, leaving 26 patients for analysis. Patient characteristics are depicted in Table 9.1.

Table 9.1 Patient characteristics.

\begin{tabular}{|c|c|}
\hline \multicolumn{2}{|l|}{ Cohort of 26 patients at inclusion } \\
\hline Male / Female & $11 / 15$ \\
\hline Age & 42 yr (SD 25) \\
\hline Body mass index & 21.8 (SD 3.7) \\
\hline Duration of disease & 11 yr (SD 10) \\
\hline CD / UC / IC & $18 / 7 / 1$ \\
\hline Truelove-Witts index ( $\mathrm{n}=7)$ (median) & 0 (range $0-1$ ) \\
\hline Harvey-Bradshaw index $(n=18)$ (median) & 1 (range $0-4$ ) \\
\hline \multirow[t]{3}{*}{ Behaviour of $C D(n=18)$} & Inflammatory = 14 \\
\hline & Stenosis $=2$ \\
\hline & Fistulising $=3$ \\
\hline AZA / 6-MP therapy & $20 / 6$ \\
\hline \multirow[t]{2}{*}{ Daily dosage } & $\mathrm{AZA}=151 \mathrm{mg}(\mathrm{SD} 38)$ \\
\hline & $6-\mathrm{MP}=63 \mathrm{mg}(\mathrm{SD} 21)$ \\
\hline \multirow[t]{2}{*}{ Dosage per kg bodyweight } & $\mathrm{AZA}=2.00 \mathrm{mg} / \mathrm{kg}(\mathrm{SD} 0,34)$ \\
\hline & $6-\mathrm{MP}=0.85 \mathrm{mg} / \mathrm{kg}(\mathrm{SD} 0,53)$ \\
\hline Duration of thiopurine use & 46 months (SD 32) \\
\hline
\end{tabular}

\section{Pharmacokinetic outcomes}

\section{Baseline ( $T=1)$}

Before initiation of 5-ASA (T=1), the median 6-TGN and 6-MMPR levels were $201 \mathrm{pmol} / 8 \times 10^{8} \mathrm{RBC}$ (range 0-601, mean $243 \mathrm{pmol} / 8 \times 10^{8} \mathrm{RBC}$ ) and 1,302 pmol $/ 8 \times 10^{8}$

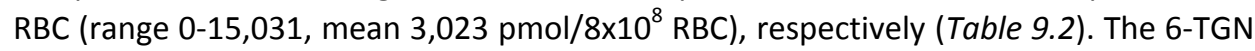
or 6-MMPR levels were not correlated with the absolute thiopurine dosage or the relative dosage per kilogram bodyweight ( $p>0.05)$. As expected, no 5-ASA or $\mathrm{N}$-acetyl5-ASA was detected in the serum of any patient. 


\section{4 weeks of 2 g 5-ASA (T=2)}

After 4 weeks of $2 \mathrm{~g} 5$-ASA per day $(\mathrm{T}=2)$, the median 6 -TGN level increased significantly to $316 \mathrm{pmol} / 8 \times 10^{8} \mathrm{RBC}$ (range 69-717, mean $326 \mathrm{pmol} / 8 \times 10^{8} \mathrm{RBC}$ ) ( $\mathrm{p}=0.001$ ) (Figure 9.3). An elevation of 6-TGN levels was observed in $88 \%$ of patients: the mean relative increase was $40 \%$, which is equivalent to an absolute rise of $84 \mathrm{pmol} / 8 \times 10^{8}$ RBC.

Table 9.2 Thiopurine and 5-ASA metabolites.

\begin{tabular}{|c|c|c|c|c|}
\hline & $\begin{array}{l}\mathrm{T}=1 \\
(0 \mathrm{~g} / \text { day } 5-\mathrm{ASA})\end{array}$ & $\begin{array}{l}\mathrm{T}=2 \\
(2 \mathrm{~g} / \text { day } 5-\mathrm{ASA})\end{array}$ & $\begin{array}{l}\mathrm{T}=3 \\
\text { (4 g/day 5-ASA) }\end{array}$ & $\begin{array}{l}T=4 \\
(0 \mathrm{~g} / \text { day } 5-A S A)\end{array}$ \\
\hline 6-TGN & $\begin{array}{l}201 \\
(0-601)\end{array}$ & $\begin{array}{l}316 \\
(69-717)\end{array}$ & $\begin{array}{l}354 \\
(103-926)\end{array}$ & $\begin{array}{l}239 \\
(0-731)\end{array}$ \\
\hline 6-MMPR & $\begin{array}{l}1,302 \\
(0-15,031)\end{array}$ & $\begin{array}{l}1,258 \\
(0-18,831)\end{array}$ & $\begin{array}{l}1,013 \\
(0-21,933)\end{array}$ & $\begin{array}{l}1,321 \\
(0-14,526)\end{array}$ \\
\hline 5-ASA & 0 & $\begin{array}{l}319 \\
(6-6,546)\end{array}$ & $\begin{array}{l}1,222 \\
(4-10,912)\end{array}$ & 0 \\
\hline N-Ac-5-ASA & 0 & $\begin{array}{l}1,706 \\
(116-6,241)\end{array}$ & $\begin{array}{l}2,861 \\
(47-9,426)\end{array}$ & 0 \\
\hline
\end{tabular}

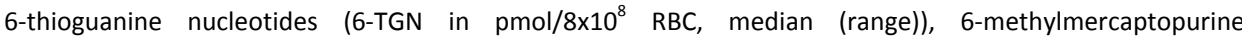
ribonucleotides (6-MMPR in $\mathrm{pmol} / 8 \times 10^{8} \mathrm{RBC}$, median (range)), 5-Aminosalicylate (5-ASA in $\mathrm{ng} / \mathrm{ml}$ serum, median (range)), $\mathrm{N}$-acetyl-5-aminosalicylic acid (N-Ac-5-ASA in $\mathrm{ng} / \mathrm{ml}$ serum, median (range)).

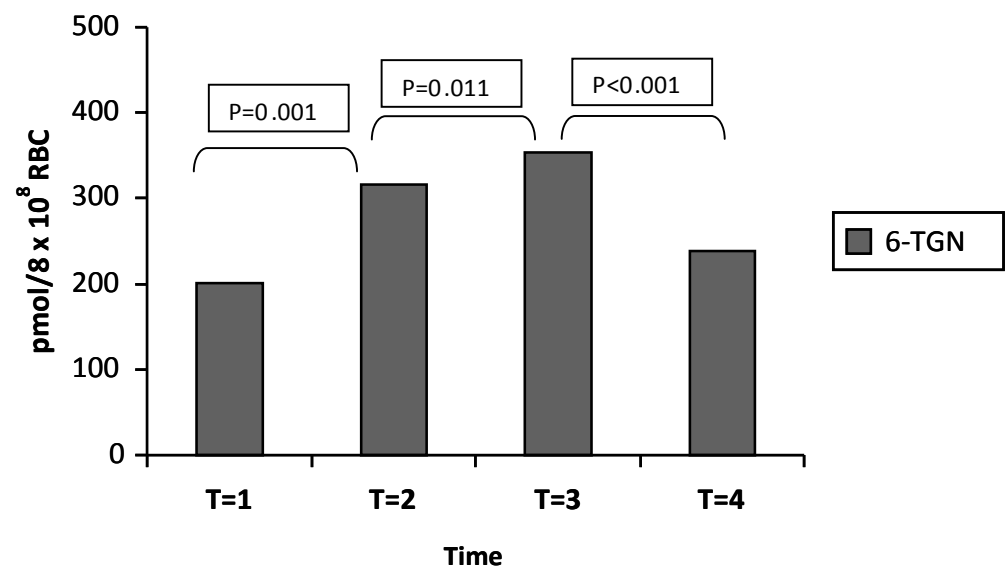

Figure 9.3 6-TGN levels during 5-ASA coadministration.

6-TGN, 6-thioguanine nucleotides.

The rise in 6-TGN levels was not dependent on 5-ASA (median $319 \mathrm{ng} / \mathrm{ml}$ serum, range 6-6,546, mean 1,055) ( $>>0.05$ ) or $\mathrm{N}$-acetyl-5-ASA serum levels (median 1,706 ng/ml serum, range $116-6,241$, mean 2,287 ) ( $p>0.05$ ), but only on the 5-ASA dosage itself. 


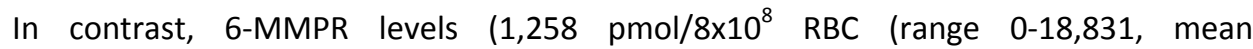
$3,516 \mathrm{pmol} / 8 \times 10^{8} \mathrm{RBC}$ )) did not change significantly (Figure 9.4).

No correlations were observed between thiopurine and 5-ASA metabolites.

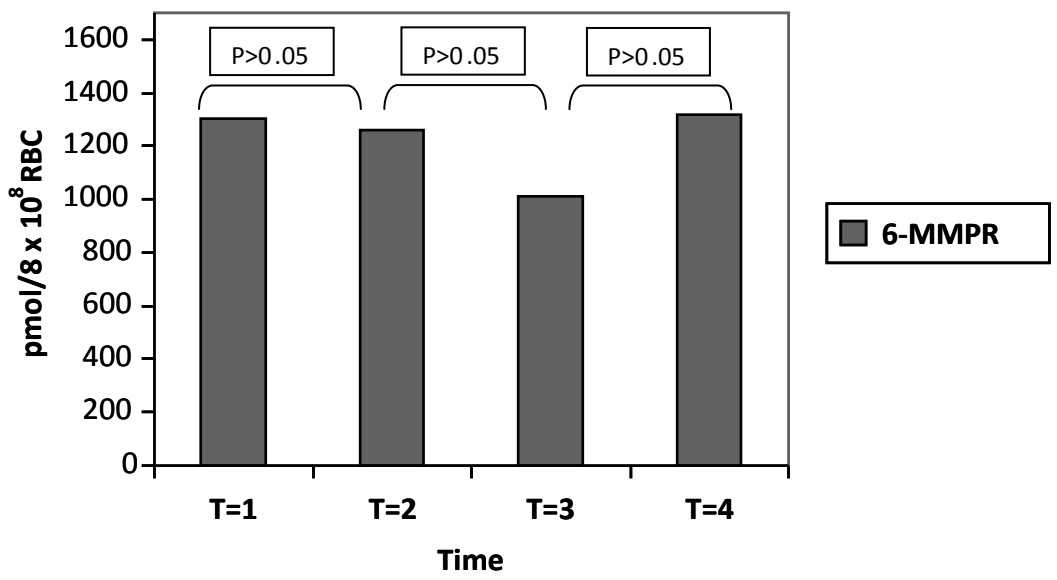

Figure 9.4 6-MMPR levels during 5-ASA coadminstration.

6-MMPR, 6-methylmercaptopurine ribonucleotides.

\section{4 weeks of $4 \mathrm{~g}$ 5-ASA (T=3)}

After 4 weeks of $4 \mathrm{~g}$ 5-ASA per day $(\mathrm{T}=3)$, the median 6-TGN levels (median $354 \mathrm{pmol} / 8 \times 10^{8} \mathrm{RBC}$, range 103-926, mean 396) increased significantly compared to $T=2(p=0.011)$ and $T=1 \quad(p<0.01)$. When compared to baseline $(T=1)$, an elevation in 6-TGN levels was observed in $100 \%$ of patients: the mean relative increase was $70 \%$, which is equivalent to an absolute rise of $154 \mathrm{pmol} / 8 \times 10^{8} \mathrm{RBC}$. When compared to $\mathrm{T}=2$, an elevation in 6-TGN levels was observed in $85 \%$ of patients: the mean relative increase was $31 \%$, which is equivalent to an absolute rise of $70 \mathrm{pmol} / 8 \times 10^{8} \mathrm{RBC}$.

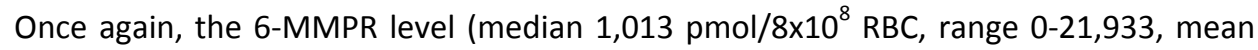
$2,896)$ did not change significantly compared to $T=2$ and $T=1$ ( $p>0.05)$.

Once again, the rise in 6-TGN level was not dependent on 5-ASA (median 1,222 ng/ml serum, range 4-10,912, mean 2,422) ( $p>0.05$ ) or $\mathrm{N}$-acetyl-5-ASA serum levels (median $2,861 \mathrm{ng} / \mathrm{ml}$ serum, range $47-9,426$, mean 3,822 ) ( $p>0.05)$, but was only dependent on the 5-ASA dosage itself.

The 5-ASA ( $p<0.01)$ and $\mathrm{N}$-acetyl-5-ASA levels $(p<0.01)$ increased significantly when compared to $T=2$. No correlations were observed between thiopurine and 5-ASA metabolites. 


\section{At least 4 weeks without 5-ASA (T=4)}

At least 4 weeks after cessation of 5-ASA therapy $(T=4)$, the 6 -TGN level (median $239 \mathrm{pmol} / 8 \times 10^{8} \mathrm{RBC}$, range $0-731$, mean 286 ) significantly decreased compared to $\mathrm{T}=3$ $(p<0.001)$. A fall of 6 -TGN levels was observed in $88 \%$ of patients: mean relative decrease was $28 \%$, which is equivalent to an absolute decrease of $110 \mathrm{pmol} / 8 \times 10^{8} \mathrm{RBC}$. The 6 -TGN level was higher at $\mathrm{T}=4$ in $88 \%$ of patients (mean relative increase $22 \%$, with a mean absolute increase of $43 \mathrm{pmol} / 8 \times 10^{8} \mathrm{RBC}$ ) when compared to $\mathrm{T}=1$; this difference was not statistically significant $(p=0.069)$.

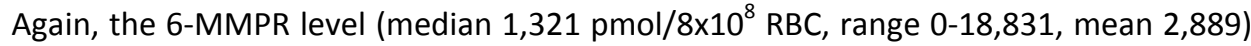
did not change significantly.

Not unexpectedly, no 5-ASA nor $\mathrm{N}$-acetyl-5-ASA were detected in the serum of any patient.

\section{Ratio of 6-MMPR and 6-TGN levels}

A 6-MMPR/6-TGN ratio below 11 correlates with clinical response to 6-MP therapy. ${ }^{4}$ In our study, the mean 6-MMPR/6-TGN ratio outcomes decreased significantly during 5-ASA coadministration. At $T=1$, the mean 6-MMPR/6-TGN ratio was 14.1 (SD 19.4, median 7.3, and range: $0-83$ ). A 6-MMPR/6-TGN ratio below 11 was observed in $60 \%$ of patients.

The 6-MMPR/6-TGN ratio (mean ratio 12.6, SD 20.6) did not significantly decrease after 4 weeks of 2 g 5-ASA ( $T=2)$ ( $p>0.05)$. A ratio below 11 was observed in $65 \%$ of patients. After 4 weeks of $4 \mathrm{~g}$ 5-ASA (T=3), the mean 6-MMPR/6-TGN ratio significantly decreased to 8.1 (SD 14.5) compared to $T=1(p=0.021)$ and $T=2(p=0.012)$. A 6-MMPR/ 6 -TGN ratio below 11 was observed in $77 \%$ of patients on $\mathrm{T}=3$.

After cessation of 5-ASA administration ( $T=4)$, the mean 6-MMPR/6-TGN ratio significantly increased (ratio 16.5, SD 16.5, median 3.3, range: $0-73$ ) compared to $T=3$ $(p=0.011)$. A 6-MMPR/6-TGN ratio below 11 was observed in $68 \%$ of patients on $T=4$.

No significant difference in mean ratio outcome was observed between $T=1$ and $T=4$ $(p=0.224)$.

\section{Laboratory parameters and adverse events}

Leukocyte and platelet counts did not change significantly during the different 5-ASA dosages. However, two patients developed a temporary leukopenia (2/26 patients $(7.7 \%))$, which resolved without cessation of 5-ASA. The first patient developed leukopenia (leukocyte count of $2.4 \times 10^{9} / \mathrm{l}$ ) after 4 weeks of $2 \mathrm{~g} 5$-ASA daily (T=2) with a corresponding 6-TGN level of $380 \mathrm{pmol} / 8 \times 10^{8} \mathrm{RBC}$, despite an increase in the 6-TGN level on $\mathrm{T}=3\left(520 \mathrm{pmol} / 8 \times 10^{8} \mathrm{RBC}\right)$ the leukocyte count increased to $3.2 \times 10^{9} / \mathrm{l}$. The second patient developed a leukocyte count of $2.4 \times 10^{9} / /$ after 4 weeks of $4 \mathrm{~g} 5$-ASA ( $\mathrm{T}=3$ ) with a corresponding 6-TGN level of $349 \mathrm{pmol} / 8 \times 10^{8} \mathrm{RBC}$ (Table 9.3). 
The erythrocyte count decreased significantly on $T=3$, compared to $T=1(p=0.003)$ and $T=2(p=0.037)$. The MCV increased significantly throughout the study: $T=1$ versus $T=2$ $(p=0.024), T=1$ versus $T=3(p<0.001), T=2$ versus $T=3(p=0.003)$ and $T=1$ versus $T=4$ $(p<0.001)$. The erythrocyte and MCV outcomes were not correlated with 6-TGN levels. During the entire study period neither laboratory nor clinical signs of hepato-, pancreatico- or nephrotoxicity were observed.

Five patients complained at one time during the study of mild gastrointestinal complaints (e.g. flatulence, nausea or change in stool frequency) possibly related to the 5-ASA administration. One patient complained of an increase of fatigue after cessation of 5-ASA.

Table 9.3 Laboratory parameters

\begin{tabular}{|c|c|c|c|c|}
\hline & $\begin{array}{l}\mathrm{T}=1 \\
(0 \mathrm{~g} / \text { day } 5-\mathrm{ASA})\end{array}$ & $\begin{array}{l}\mathrm{T}=2 \\
(2 \mathrm{~g} / \text { day } 5-\mathrm{ASA})\end{array}$ & $\begin{array}{l}\mathrm{T}=3 \\
(4 \mathrm{~g} / \text { day } 5-\mathrm{ASA})\end{array}$ & $\begin{array}{l}T=4 \\
(0 \mathrm{~g} / \text { day } 5-A S A)\end{array}$ \\
\hline Leukocytes & 5.1 (SD 1.5) & 5.1 (SD 1.8) & 4.7 (SD 1.7) & 5.5 (SD 1.9) \\
\hline Platelets & 287 (SD 75) & 293 (SD 74) & 296 (SD 97) & 274 (SD 70) \\
\hline Erythrocytes & 4.3 (SD 0.4) & 4.2 (SD 0.5) & 4.1 (SD 0.5) & 4.2 (SD 0.4) \\
\hline MCV & 93 (SD 6) & 94 (SD 6) & 95 (SD 6) & 96 (SD 6) \\
\hline $\mathrm{Hb}$ & 8.4 (SD 0.8) & 8.3 (SD 0.8) & 8.3 (SD 0.8) & 8.5 (SD 0.7) \\
\hline ASAT & 25 (SD 10) & 25 (SD 8) & 24 (SD 10) & 25 (SD 11) \\
\hline ALAT & 27 (SD 21) & 27 (SD 18) & 27 (SD 20) & 27 (SD 23) \\
\hline AP & 76 (SD 26) & 73 (SD 23) & 72 (SD 21) & 71 (SD 22) \\
\hline GGT & 23 (SD 21) & 23 (SD 22) & 23 (SD 21) & 23 (SD 22) \\
\hline Amylase & 124 (SD 52) & 126 (SD 47) & 133 (SD 79) & 125 (SD 44) \\
\hline Albumin & 42 (SD 3) & 42 (SD 3) & 42 (SD 3) & 42 (SD 3) \\
\hline Creatinin & 88 (SD 11) & 88 (SD 10) & 87 (SD 10) & 88 (SD 12) \\
\hline
\end{tabular}

Mean cellular volume (MCV), haemoglobin $(\mathrm{Hb})$, aspartate transaminase (ASAT), alanine transaminase (ALAT), alkaline phosphatase (AP), gammaglutamyl transferase (GGT).

\section{Discussion}

This prospective pharmacokinetic study shows for the first time that the level of the pharmacologically active thiopurine metabolites 6-TGN significantly increases in a dosedependent manner during 5-ASA coadministration in IBD patients. The 4-weeks coadministration of $2 \mathrm{~g}$ 5-ASA daily, followed by a 4-weeks period of $4 \mathrm{~g}$ 5-ASA daily, during steady maintainance thiopurine therapy, led to an increase of $40 \%$ (absolute $84 \mathrm{pmol} / 8 \times 10^{8} \mathrm{RBC}$ ) and $70 \%$ (absolute $154 \mathrm{pmol} / 8 \times 10^{8} \mathrm{RBC}$ ) in 6-TGN levels, respectively. The different 5-ASA dosages had no significant influence on the 6-MMPR levels, which indicates that the in vivo activity of the methylating enzyme TPMT is not 
influenced by 5-ASA or its metabolites, when 5-ASA is administered in dosages currently used in daily practice. This is in contrast to several in vitro studies that have demonstrated an inhibition of TPMT by 5-ASA compounds. Our findings are in agreement with a recent prospective study showing that the TPMT activity was not significantly affected by mesalazine monotherapy during a whole year of treatment. ${ }^{15}$

Thiopurine metabolism in humans is complex, resulting in a broad range of metabolites, of which only those considered to be of pivotal importance with regard to prediction of efficacy and toxicity, 6-TGN and 6-MMPR, were assessed. This limits the number of hypotheses that can be put forward to explain the observed interaction between thiopurines and 5-ASA. Firstly, the bioavailablity of thiopurines may be enlarged during 5-ASA coadminstration due to increased intestinal absorption. However, this would lead to an increase in both 6-TGN and 6-MMPR levels. Therefore, it is unlikely that the bioavailability of thiopurines changes during 5-ASA coadministration. Secondly, 5-ASA compounds may lead to an inhibition or enhancement of the activity of enzymes responsible for thiopurine metabolism (Figure 9.1). An enhancement of the enzymes inosine monophosphate dehydrogenase (IMPDH) or guanosine monophosphate synthetase (GMPS) may provide an explanation. These enzymes transform 6-thioinosine monophosphate (6-TIMP) into 6-TGN, during these two steps no 6-MMP or 6-MMPR is generated. Another explanation concerns the possible influence of 5-ASA compounds on the activity of multidrug-resistance transporters, especially multidrugresistance protein 4 and $5 .{ }^{16}$ Theoretically, an inhibition of the activity of 6-TGN-efflux transporters by 5-ASA will lead to a rise in 6-TGN concentrations. However, definitive conclusions regarding this potential interaction can not be drawn from our study design and results.

We can conclude that the rise in 6-TGN levels is dependent on the 5-ASA dosage but the separate 5-ASA metabolite concentrations itself do not play a crucial role in the pharmacokinetic interaction between 5-ASA and thiopurines, because no correlations were observed. The influence of 5-ASA on thiopurine metabolism is only temporary and reversible, as after withdrawal of 5-ASA the 6-TGN levels decreased towards the baseline outcomes.

The results of our study may be influenced by the delayed-release microgranular form of the 5-ASA compound administered to our patients, and the observed changes in thiopurine metabolism may be dependent on the release and absorption characteristics of this 5-ASA preparation.

During our study, signs of myelotoxicity were observed in $7.7 \%$ of patients $(n=2)$ during the combination of 5-ASA and thiopurine therapy. Our results are in accordance with the incidence of leukopenia (10\%) reported in a study by Lowry et al. ${ }^{11}$ Remarkably, during their leukopenia both patients in our study had a 6-TGN level below $490 \mathrm{pmol} / 8 \times 10^{8} \mathrm{RBC}$, which has been proposed as the upper reference level associated 
with therapeutic efficacy. ${ }^{3}$ Therefore, another explanation for the induction of leukopenia during 5-ASA and thiopurine therapy, besides elevated 6-TGN levels, is likely. Importantly, we note that myelosuppression is also a rare adverse effect of 5-ASA itself, so that combination may lead to an additive myelotoxic effect. In our study, we did not find a significant change in leukocyte and platelet counts during the different 5-ASA dosages. Although a significant decrease in erythrocyte count and rise in MCV, not explained by 6-TGN levels, were observed. Our results emphasise the need for close monitoring of patients during 5-ASA and thiopurine therapy as there appears to be an increased risk of developing myelotoxicity, especially after initiation of this combination therapy or after changes in 5-ASA dosages.

The observed pharmacokinetic influence of 5-ASA on thiopurine metabolism, leading to an increase in only 6-TGN levels may have, besides the changed toxicity profile, important clinical implications. Patients refractory or unresponsive to thiopurine therapy may benefit from 5-ASA coadminstration as higher 6-TGN levels have been associated with a better response to thiopurine therapy. ${ }^{17}$ In addition, the 6-MMPR/6-TGN ratio decreased during 5-ASA coadminstration, indicating an improvement of metabolite profiles and associated with potentiation of thiopurine efficacy. ${ }^{4}$ However, our study was not designed to draw any firm conclusions about therapeutic efficacy but it seems promising to administer 5-ASA compounds in IBD patients who do not benefit from thiopurine therapy alone or have developed inadequate 6-TGN levels (below $235 \mathrm{pmol} / 8 \times 10^{8} \mathrm{RBC}$ ). In clinical practice, our findings could also be beneficial in patients with preferential shunting to potentially hepatotoxic 6-MMPR levels, often resulting in low and clinically inadequate 6-TGN levels. This pharmacological approach constitutes an alternative way to an increase in AZA or 6-MP dosages in order to optimise 6-TGN levels, as this will inevitably lead to a concomitant increase of (potentially toxic) 6-MMPR levels.

In conclusion, introduction of 5-ASA during steady-state thiopurine therapy induces a dose-dependent increase of 6-TGN levels ( $2 \mathrm{~g} 40 \%$ and $4 \mathrm{~g} 70 \%$ increase) but has no effect on 6-MMPR levels. This finding results from synergistic pharmacological effects of 5-ASA on thiopurine metabolism. The underlying mechanism is still unknown. Expectedly, this combination of drugs is associated with a slightly increased risk of developing myelotoxicity (7.7\%). Clinicians should be aware of this higher risk for myelosuppression. IBD patients who are unresponsive or refractory to standard thiopurine therapy may benefit from the coadministration of 5-ASA leading to an increase in the pharmacologically active 6-TGN levels. ${ }^{17}$ 


\section{References}

1. Al Hadithy AF, de Boer NK, Derijks LJ, Escher JC, Mulder CJ, Brouwers JR. Thiopurines in inflammatory bowel disease: pharmacogenetics, therapeutic drug monitoring and clinical recommendations. Dig Liver Dis 2005;37:282-97.

2. Neurath MF, Kiesslich R, Teichgräber U, Fischer $C$, Hofmann U, Eichelbaum M, Galle PR, Schwab M. 6-thioguanosine diphosphate and triphosphate levels in red blood cells and response to azathioprine therapy in Crohn's disease. Clin Gastroenterol Hepatol 2005;3:1007-14.

3. Dubinsky MC, Lamothe S, Yang HY, Targan SR, Sinnett D, Théorêt Y, Seidman EG. Pharmacogenomics and metabolite measurement for 6-mercaptopurine therapy in inflammatory bowel disease. Gastroenterology 2000;118:705-13.

4. Dubinsky MC, Yang H, Hassard PV, Seidman EG, Kam LY, Abreu MT, Targan SR, Vasiliauskas EA. 6-MP metabolite profiles provide a biochemical explanation for 6-MP resistance in patients with inflammatory bowel disease. Gastroenterology 2002;122:904-15.

5. Van Bodegraven AA, Mulder CJ. Indications for 5-aminosalicylate in inflammatory bowel disease: is the body of evidence complete?. World J Gastroenterol 2006;12:6115-23.

6. Szumlanski $\mathrm{CL}$, Weinshilboum RM. Sulphasalazine inhibition of thiopurine methyltransferase: possible mechanism for interaction with 6-mercaptopurine and azathioprine. Br J Clin Pharmacol 1995;39: 456-9.

7. Dewit O, Vanheuverzwyn R, Desager JP, Horsmans Y. Interaction between azathioprine and aminosalicylates: an in vivo study in patients with Crohn's disease. Aliment Pharmacol Ther 2002;16: 79-85.

8. Xin H, Fischer C, Schwab M, Klotz U. Effects of aminosalicylates on thiopurine S-methyltransferase activity: an ex vivo study in patients with inflammatory bowel disease. Aliment Pharmacol Ther 2005;21:1105-9.

9. Hande S, Wilson-Rich N, Bousvaros A, Zholudev A, Maurer R, Banks P, Makrauer F, Reddy S, Burakoff R, Friedman S. 5-Aminosalicylate therapy is associated with higher 6-thioguanine levels in adults and children with inflammatory bowel disease in remission on 6-mercaptopurine or azathioprine. Inflamm Bowel Dis 2006;12:251-7.

10. Gilissen LP, Bierau J, Derijks LJ, Bos LP, Hooymans PM, van Gennip A, Stockbrügger RW, Engels LG. The pharmacokinetic effect of discontinuation of mesalazine on mercaptopurine metabolite levels in inflammatory bowel disease patients. Aliment Pharmacol Ther 2005;22:605-11.

11. Lowry PW, Franklin CL, Weaver AL, Szumlanski CL, Mays DC, Loftus EV, Tremaine WJ, Lipsky JJ, Weinshilboum RM, Sandborn WJ. Leucopenia resulting from a drug interaction between azathioprine or 6-mercaptopurine and mesalamine, sulphasalazine, or balsalazide. Gut 2001;49:656-64.

12. Lewis LD, Benin A, Szumlanski CL, Otterness DM, Lennard L, Weinshilboum RM, Nierenberg DW. Olsalazine and 6-mercaptopurine-related bone marrow suppression: a possible drug-drug interaction. Clin Pharmacol Ther 1997;62:464-75.

13. Derijks LJ, Gilissen LP, Engels LG, Bos LP, Bus PJ, Lohman JJ, Curvers WL, Van Deventer SJ, Hommes DW, Hooymans PM. Pharmacokinetics of 6-mercaptopurine in patients with inflammatory bowel disease: implications for therapy. Ther Drug Monit 2004;26:311-8.

14. Hussain FN, Ajjan RA, Moustafa M, Anderson JC, Riley SA. Simple method for the determination of 5-aminosalicylic and $\mathrm{N}$-acetyl-5-aminosalicylic acid in rectal tissue biopsies. J Chromatogr. B Biomed Sci Appl 1998; 716; 257-66.

15. Dilger K, Schaeffeler E, Lukas M, Strauch U, Herfarth H, Müller R, Schwab M. Monitoring of thiopurine methyltransferase activity in postsurgical patients with Crohn's disease during 1 year of treatment with azathioprine or mesalazine. Ther Drug Monit 2007;29:1-5.

16. van der Heijden JW, Dijkmans BA, Scheper RJ, Jansen G. Drug Insight: resistance to methotrexate and other disease-modifying antirheumatic drugs-from bench to bedside. Nat Clin Pract Rheumatol 2007;3:26-34.

17. Osterman MT, Kundu R, Lichtenstein GR, Lewis JD. Association of 6-thioguanine nucleotide levels and inflammatory bowel disease activity: a meta-analysis. Gastroenterology 2006;130:1047-53. 


\section{Chapter 10}

Influence of 5-aminosalicylic acid on 6-thioguanosine phosphate metabolite levels: a prospective study in patients under steady thiopurine therapy

P de Graaf, NKH de Boer, DR Wong, S Karner, B Jharap, PM Hooymans, Al Veldkamp, CJJ Mulder, AA van Bodegraven, M Schwab

Br J Pharmacol. 2010;160:1083-1091 


\section{Abstract}

Background and purpose

5-aminosalicylate (5-ASA) raises levels of 6-thioguanine nucleotides (6-TGN), the active metabolites of thiopurines such as azathioprine (AZA). Changes in levels of each individual 6TGN - 6-thioguanosine mono-, di- and triphosphate (6-TGMP, 6-TGDP, 6-TGTP) - and of 6-methylmercaptopurine ribonucleotides (6-MMPR) after 5-ASA are not known.

Experimental approach

Effects of increasing 5-ASA doses on AZA metabolites were investigated prospectively in 22 patients with inflammatory bowel disease in 4-week study periods. Patients started with $2 \mathrm{~g}$ 5-ASA daily, and then were increased to $4 \mathrm{~g}$ daily and followed by a washout period. Thiopurine doses remained unchanged throughout the entire study. Levels of 6-TGMP, 6-TGDP, 6-TGTP and 6-MMPR as well as of 5-ASA and $\mathrm{N}$-acetyl-5-aminosalicylic acid (N-Ac-5-ASA) were determined each study period.

Key results

Median baseline levels in 17 patients of 6-TGDP, 6-TGTP and 6-MMPR were 52, 319 and $1,676 \mathrm{pmol} / 8 \times 10^{8}$ red blood cells, respectively. After co-administration of $2 \mathrm{~g} 5$-ASA daily, median 6-TGDP and 6-TGTP levels increased but median 6-MMPR levels were unchanged. Increasing 5-ASA to $4 \mathrm{~g}$ daily did not affect median 6-TGDP and 6-TGTP levels, but median 6-MMPR levels decreased. After discontinuation of 5-ASA, both 6-TGDP and 6-TGTP levels decreased and median 6-MMPR levels increased. The 6-TGTP/(6-TGDP+6-TGTP)-ratio did not change during the study, but 6-MMPR/6-TGN ratios decreased.

Conclusions and implications

Individual 6-TGN metabolites increased after addition of 5-ASA, but 6-MMPR-levels and the 6-MMPR/6-TGN ratios decreased. Further studies are needed to decide whether this pharmacokinetic interaction would result in improvement of efficacy and/or increased risk of toxicity of AZA. 


\section{Introduction}

Azathioprine (AZA) and 6-mercaptopurine (6-MP) are immunosuppressive drugs used for treatment of ulcerative colitis (UC), Crohn's disease (CD) and indeterminate colitis (IC). ${ }^{1}$ Their metabolism is complex (Figure 10.1) and involves in part polymorphically expressed drug metabolising enzymes such as thiopurine S-methyltransferase (TPMT) and inosine triphosphate pyrophosphatase (ITPase). The metabolic pathway ultimately results in formation of 6-thioguanine nucleotides (6-TGN) and methylated compounds (e.g. 6-methylmercaptopurine ribonucleotides, 6-MMPR). ${ }^{2}$ Pharmacological activity of thiopurines in inflammatory bowel diseases (IBD) is attributed to the specific metabolite 6-thioguanosine-triphosphate (6-TGTP), which is the final product of successive intracellular phosphorylation of 6-thioguanosine mono- and 6-thioguanosine diphosphate (6-TGMP; 6-TGDP). Tiede et al. revealed that binding of 6-TGTP to the GTPase Rac1 resulted in a mitochondrial pathway of apoptosis in CD3-CD28 costimulated T cells. ${ }^{3}$ In detail, 6-TGTP primarily blocks the guanosine exchange factor Vav1, leading to accumulation of inactive 6-TGDP-loaded Rac1 and subsequent suppression of CD-28 lamellipodia formation and interferon (IFN)- $\nu$ production. ${ }^{4}$

In IBD, optimal therapeutic response has been associated with 6-TGN levels above $235 \mathrm{pmol} / 8 \times 10^{8}$ red blood cells (RBC), although 6-TGN levels higher than $490 \mathrm{pmol} / 8 \times 10^{8} \mathrm{RBC}$ are associated with an increased risk to develop myelotoxicity.

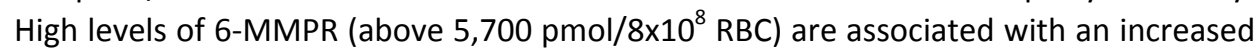
risk for hepatotoxicity, but also with treatment failure. ${ }^{5,6}$ A 6-MMPR/6-TGN ratio lower than 11 is suggested to be indicative of a good response to AZA/6-MP therapy, whereas a ratio higher than 11 appears to be an indicator for failure of AZA/6-MP therapy, even after dose escalation. ${ }^{7}$ Routine therapeutic monitoring of 6-TGN and 6-MMPR in RBC during thiopurine therapy is advocated to optimise thiopurine therapy and verify therapeutic compliance. ${ }^{5,8}$ Nevertheless, the risk of developing adverse events requires frequent monitoring of routine laboratory parameters.

In addition to thiopurines, 5-aminosalicylates (5-ASA) is also frequently used in treatment of IBD. The exact pharmacological mechanism of 5-ASA is unknown, although a local anti-inflammatory effect is considered. ${ }^{9}$ After absorption, 5-ASA is metabolised to the major inactive metabolite $\mathrm{N}$-acetyl-5-aminosalicylate ( $\mathrm{N}-\mathrm{Ac}-5-\mathrm{ASA}$ ) by the $\mathrm{N}$-acetyl-transferase-1 enzyme in intestinal epithelial and liver cells. ${ }^{10,11}$

Various studies have reported elevated 6-TGN levels, due to an alteration in thiopurine metabolism probably caused by coadministration of 5-ASA during thiopurine therapy. This increase has been associated with a higher risk for leukopenia, but also improved efficacy. ${ }^{12-18}$ The underlying pharmacological mechanism remains to be verified. It has been suggested that TPMT activity may be inhibited by 5-ASA, shunting metabolite formation from 6-MMPR towards 6-TGN, ${ }^{12-14}$ but the data are in part contradictory. ${ }^{19}$

Recently, we have published a prospective pharmacokinetic interaction study in IBD patients on steady state maintenance AZA or 6-MP therapy. ${ }^{18} \mathrm{~A}$ dose-dependent effect was observed for two different 5-ASA dosages ( $2 \mathrm{~g}$ followed by $4 \mathrm{~g}$, both daily during 
4 weeks) on total levels of 6-TGN and 6-MMPR metabolites. Total 6-TGN levels increased significantly whereas 6-MMPR metabolite levels tended to decrease. However, the effect of 5-ASA on levels of the individual nucleotides (6-TGMP, 6-TGDP and the pivotal 6-TGTP) is currently unknown. Therefore, the present study aimed to determine the influence of 5-ASA on levels of 6-TGMP, 6-TGDP and 6-TGTP as well as 6-MMPR, using a subcohort of the previously described study population.

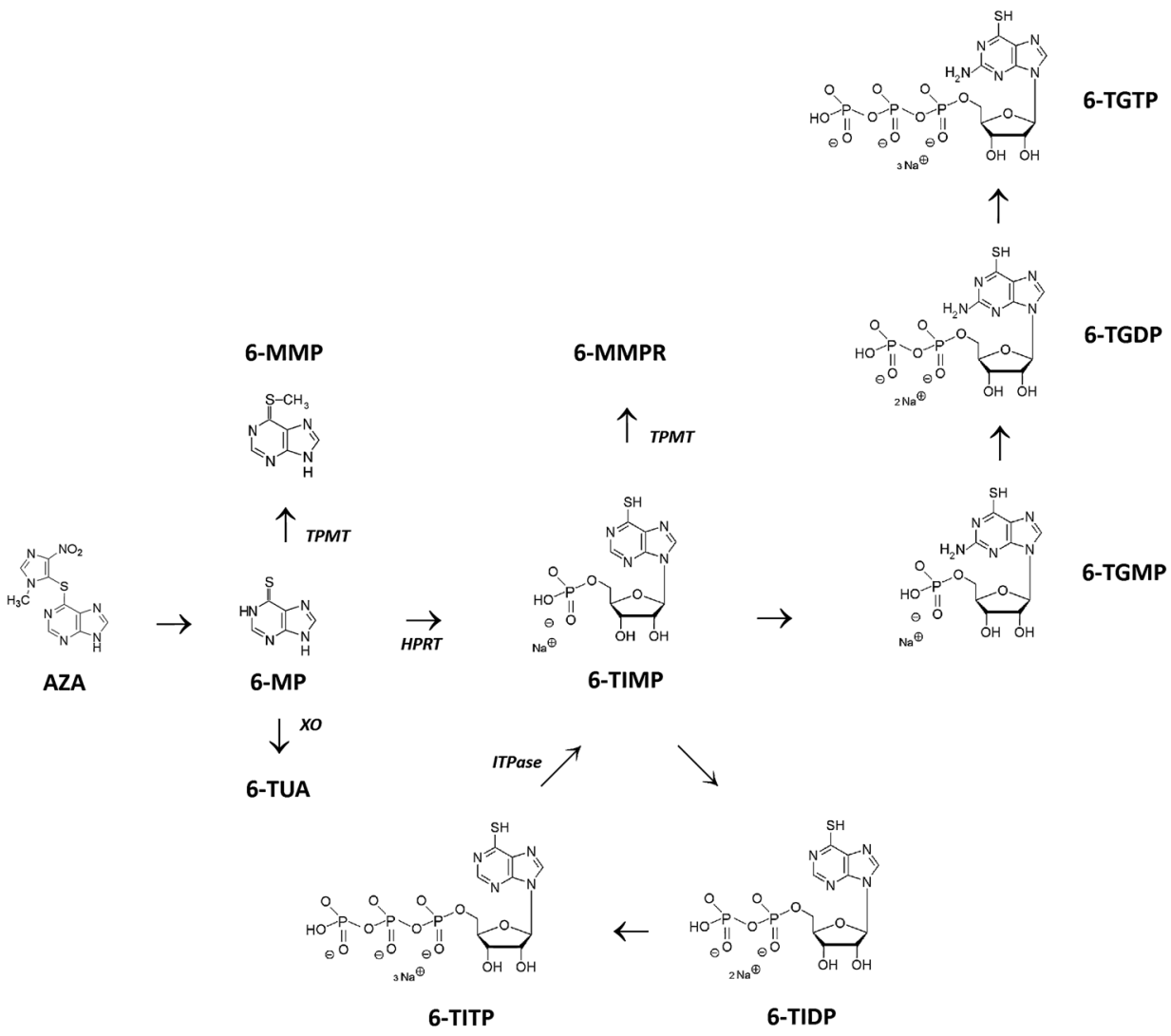

Figure 10.1 Thiopurine metabolism (simplified).

Azathioprine (AZA) is non-enzymatically degraded to 6-mercaptopurine (6-MP). Xanthine oxidase inactivates 6-MP by the formation of 6-thiouric acid (6-TUA). Thiopurine S-methyltransferase (TPMT) methylates 6-MP into 6-methylmercaptopurine (6-MMP). Via hypoxanthine phosphoribosyl transferase (HPRT), 6-MP is converted to 6-thioinosine monophosphate (6-TIMP). Via two other enzymatic steps, inosine monophosphate dehydrogenase (IMPDH) and guanosine monophosphate synthetase (GMPS), a pool of 6-thioguanine nucleotides (6-TGN) is ultimately generated, consisting of 6-thioguanine monophosphate (6-TGMP), 6-thioguanine diphosphate (6-TGDP) and 6-thioguanine triphosphate (6-TGTP). 6-TIMP may also be methylated by TPMT leading to 6-methylmercaptopurine ribonucleotides (6-MMPR) (consisting of 6-methyl-thioinosine monophosphate, 6-methyl-thioinosine diphosphate and 6-methyl-thioinosine triphosphate). In a closed cycle, 6-TIMP may be phosphorylated to 6-thioinosine diphosphate (6-TIDP), subsequently to 6-thioinosine triphosphate (6-TITP) and ultimately back to 6-TIMP via the inosine triphosphate pyrophosphatase (ITPase). 


\section{Materials and methods}

\section{Patient selection}

The study protocol was approved by the ethical committee of the VU University Medical Centre and all patients gave written consent before enrolment in the study. The detailed inclusion criteria and prospective study design have been fully described elsewhere. ${ }^{18}$ All IBD patients were on steady-state thiopurine therapy at baseline with AZA or 6-MP for at least eight consecutive weeks (T1). Study scheme consisted of $2 \mathrm{~g}$ 5-ASA once daily (T2), followed by 2 g 5-ASA twice daily (T3), both during 4 weeks with continuation of AZA or 6-MP in unchanged doses (Figure 10.2). Finally, there was a wash-out period of 4 weeks (T4). The 4-week intervals were chosen in order to reach steady-state levels of thiopurine metabolites. ${ }^{20}$ The 5-ASA was given as Pentasa microgranules (Ferring, Hoofddorp; The Netherlands). Metabolites were assessed at baseline (T1) and at the end of each 4-week study interval (T2-T4). Demographic data and disease activity (Harvey-Bradshaw index for CD and Truelove-Witts index for UC) were collected at baseline. Levels of thiopurine metabolites (6-MMPR and 6-TGMP, 6-TGDP and 6-TGTP in RBC) as well as 5-aminosalicylate levels (5-ASA- and N-Ac-5-ASA levels in serum) were determined at each study interval.

\section{Outcome measurements}

Primary outcome parameters were the changes in levels and ratios of the 6-TGMP, 6-TGDP, 6-TGTP and 6-MMPR metabolites during the different 5-ASA dosage regimes.

\section{Determination of 6-TGMP/6-TGDP/6-TGTP, 6-MMPR and 6-TGN levels in RBC}

Blood samples were collected in coated lithium-heparin tubes, homogenised immediately and divided in equal portions for analysis of 6-MMPR or 6-TGMP/ 6-TGDP/6-TGTP levels, respectively. The 6-MMPR sample was refrigerated, and subsequently sent to the laboratory of the Clinical Pharmacy \& Toxicology in the Orbis Medical Centre (Sittard-Geleen, The Netherlands) for analysis and kept frozen until analysis of the 6-MMPR levels. The 6-MMPR levels were determined using a validated assay, based on a modified method as previously described by Derijks et al.. ${ }^{20}$

The lower limit of detection (LOD) and lower limit of quantification (LOQ) of the 6-MMPR assay was found to be 150 and $300 \mathrm{pmol} / 8 \times 10^{8} \mathrm{RBC}$, respectively. Determination of 6-TGMP, 6-TGDP and 6-TGTP levels in RBC requires a strict preanalytical sample procedure since substantial interconversion between these metabolites due to phosphorylation and transphosphorylation processes in RBC has been found. Furthermore, the stability of the 6-TGN metabolites after patient sampling is critical. ${ }^{21,22}$ The second sample was therefore centrifuged immediately for isolation of RBC, subsequently washed with PBS buffer solution and counting of RBC was performed. All samples were stored at $-80^{\circ} \mathrm{C}$ until analysis. Levels of 6-TGMP, 6-TGDP 
and 6-TGTP in RBCs were determined at the Dr. Margarete Fischer-Bosch Institute of Clinical Pharmacology by a previously described high performance liquid chromatography (HPLC) assay. ${ }^{23}$

The LOD for all 6-thioguanosine phosphates (TGMP, TGDP,TGTP) was $20 \mathrm{pmol} \cdot \mathrm{ml}^{-1} \mathrm{RBC}$, corresponding to approximately $1.6 \mathrm{pmol} / 8 \times 10^{8} \mathrm{RBC}$. The LOQ for all analytes was $100 \mathrm{pmol} \cdot \mathrm{ml}^{-1}$, corresponding to approximately $8.0 \mathrm{pmol} / 8 \times 10^{8} \mathrm{RBC}$.

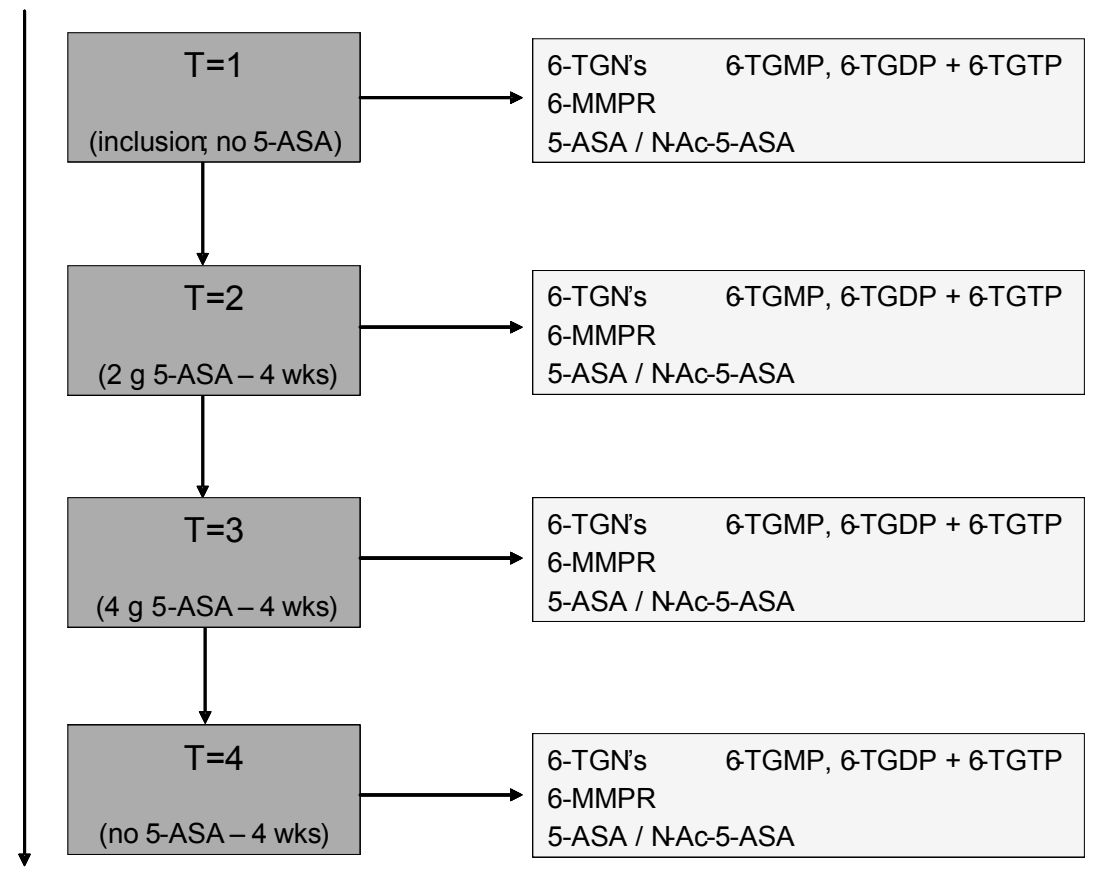

Figure 10.2 Trial design.

The effect of increasing 5-ASA doses on AZA metabolite levels was investigated in three study periods of 4 weeks. Patients started with 2 g 5-ASA daily, which was subsequently increased to $4 \mathrm{~g}$ daily and followed by a washout period. Thiopurine doses remained unchanged throughout the entire study. Levels of 6-TGMP, 6-TGDP, 6-TGTP and 6-MMPR as well as those of 5-ASA and $\mathrm{N}$-acetyl-5-aminosalicylic acid (N-Ac-5-ASA) were determined in each study period. 5-ASA, 5-aminosalicylate; 6-MMPR, 6-methylmercaptopurine ribonucleotides; 6-TGDP, 6-thioguanosine diphosphate; 6-TGMP, 6-thioguanosine monophosphate; 6-TGN, 6-thioguanine nucleotides; 6-TGTP, 6-thioguanosine triphosphate; AZA, azathioprine.

\section{Determination of 5-ASA and N-Ac-5-ASA levels}

Blood samples were collected in serum tubes. The samples were centrifuged for at least 5 minutes at $1500 \mathrm{x}$. Subsequently, the serum was separated and stored at $-20^{\circ} \mathrm{C}$ until analysis. The 5-ASA and N-Ac-5-ASA serum levels were measured in the Laboratory of Pharmacology \& Toxicology (Orbis Medical Centre, Sittard-Geleen, The Netherlands), using a slightly modified assay of Hussain et al.. ${ }^{24}$ 
The LOD for both 5-ASA and N-acetyl-5-ASA was $4 \mathrm{ng} \cdot \mathrm{mL}^{-1}$. LOQ for both 5 -ASA and $\mathrm{N}$-acetyl-5-ASA was $10 \mathrm{ng} \cdot \mathrm{mL}^{-1}$. The within-run coefficient of variation was maximally $3.3 \%$ for both $5-A S A$ and $\mathrm{N}$-acetyl-5-ASA in the range of the calibration.

\section{Statistical analysis}

Data are shown descriptively and expressed as median with range. Differences in metabolite levels, changes in 6-MMPR/6-TGN and 6-TGTP/(6-TGDP+6-TGTP) ratios were calculated and compared using the Wilcoxon Matched-Pairs Signed-Ranks Test. A mixed model analysis was used to detect parameters that influenced metabolite levels. Correlations between parameters were determined using the Pearson's or Spearman's test, when indicated. P-values of less than 0.05 were considered significant. Statistical Package for the Social Sciences (SPSS) for windows version 15.0 was used for statistical analysis.

\section{Results}

\section{Patient demographics}

Twenty-two IBD patients were included at the VU University Medical Centre. Five patients were excluded from analysis due to incomplete data (all insufficient sample material), leaving 17 patients for analysis. Patient characteristics are presented in Table 10.1. All patients used AZA with a median dose of $1.90 \mathrm{mg} / \mathrm{kg}$.

Table 10.1 Patient characteristics.

\begin{tabular}{|c|c|}
\hline Male / Female & $6 / 11(n=17)$ \\
\hline Age (median) & 41 years (range: $21-57)$ \\
\hline Body mass index (median) & 21.7 (range: 15.9-27.8) \\
\hline CD / UC / IC & $11 / 5 / 1$ \\
\hline Truelove-Witts index ( $n=5)$ (median) & 0 (range $0-1)$ \\
\hline Harvey-Bradshaw index $(n=12)$ (median) & 1 (range $0-4$ ) \\
\hline \multirow[t]{3}{*}{ Behaviour of $\mathrm{CD}(\mathrm{N}=11)$} & Inflammatory $=8$ \\
\hline & Stenotising = 1 \\
\hline & Fistulising $=2$ \\
\hline Daily dosage (median) & $A Z A=150 \mathrm{mg} /$ day (range: $100-200)$ \\
\hline Dosage per kg bodyweight (median) & $\mathrm{AZA}=1.90 \mathrm{mg} / \mathrm{kg}$ (range: $1.47-2.74)$ \\
\hline Duration of thiopurine use (median) & 59 months (range: $11-147)$ \\
\hline
\end{tabular}

CD: Crohn's disease, UC: Ulcerative colitis, IC: Indeterminate colitis, AZA: azathioprine. 


\section{Pharmacokinetic outcomes}

Median values of the individual 6-TGN, total 6-TGN, total 6-MMPR levels and 5-ASA/N-5-Ac-ASA concentrations are shown in Figure 10.3

\section{Metabolite levels at T1}

Median levels (and range) of total 6-TGN, 6-TGTP, 6-TGDP and 6-MMPR were 370 (96-682), 319 (83-583), $52(14-192)$ and 1,676 $(0-9,837) \mathrm{pmol} / 8 \times 10^{8} \mathrm{RBC}$, respectively; 6-TGMP levels were below the detection limit in all patients.

The median total 6-TGN levels consisted of 85.5\% 6-TGTP and 14.5\% 6-TGDP. All except four patients showed total 6-TGN levels higher than $235 \mathrm{pmol} / 8 \times 10^{8} \mathrm{RBC}$. As expected, no 5-ASA or N-Ac-5-ASA was detected. No correlation was found between thiopurine dose (absolute or the relative dose per $\mathrm{kg}$ bodyweight), individual and total 6-TGN or 6-MMPR levels.

\section{Metabolite levels at T2}

Co-medication with $2 \mathrm{~g}$ 5-ASA daily over 4 weeks (T2) led to a significant increase in 6-TGTP ( $p=0.0002), 6-T G D P(p=0.0002)$ and total 6-TGN $(p=0.0002)$ metabolite levels. Median levels (and range) of total 6-TGN, 6-TGTP, 6-TGDP and 6-MMPR were 553 (141-1,139), 482 (123-949), 77 (18-242) and 1,447 (0-10,412), respectively; 6-TGMP levels were below the LOQ for all patients except one subject, reaching a value marginally above LOQ $\left(123 \mathrm{pmol} \cdot \mathrm{ml}^{-1}\right)$. Serum levels of 5-ASA and N-Ac-5-ASA were 175 $(6-4,301)$ and $1,260(116-6,241) \mathrm{ng} \cdot \mathrm{ml}^{-1}$. The median relative increase in 6-TGDP, 6-TGTP and total 6-TGN-levels was $48 \%, 51 \%$ and $49 \%$, respectively, being equivalent to an absolute rise of 25, 163 and $183 \mathrm{pmol} / 8 \times 10^{8} \mathrm{RBC}$. The 6-TGTP and 6-TGDP levels formed $84 \%$ and $16 \%$ of the total 6 -TGN levels, similar to T1. Median 6-MMPR levels at T2 remained unchanged. All except three patients revealed total 6-TGN levels above $235 \mathrm{pmol} / 8 \times 10^{8} \mathrm{RBC}$. Detailed analysis showed an increase in total 6-TGN levels in 14 patients (82\%). Concerning 6-TGDP and 6-TGTP, elevation of metabolite levels was observed in 16 and 14 patients, respectively (94\% and 82\%). Although statistically insignificant $(p=0.82)$, an overall decrease in $6-M M P R$ levels of $14 \%$ was observed, representing a median decrease of $229 \mathrm{pmol} / 8 \times 10^{8} \mathrm{RBC}$. No correlations were observed between 6-TGN, 6-TGDP, 6-TGTP or 6-MMPR levels and serum 5-ASA or N-Ac-5-ASA levels at T2, nor was the rise in individual 6-TGN, 6-TGDP and 6-TGTP levels associated with serum levels of 5-ASA or N-Ac-5-ASA.

\section{Metabolite levels at T3}

Further dose escalation of 5-ASA towards $4 \mathrm{~g}$ daily (T3) did not reveal a significant change in median levels of 6-TGDP, 6-TGTP and total 6-TGN levels compared with T2 ( $p=0.404, p=0.669$ and $p=0.84$, respectively). Median levels (and range) of total 6-TGN, 
6-TGTP, 6-TGDP and 6-MMPR were 572 (184- 1,207), 483 (155-908), 89 (29-298) and $880(0-9,431)$, respectively. Again, 6-TGMP levels were below the LOQ except in one case $\left(141 \mathrm{pmol} \cdot \mathrm{ml}^{-1}\right)$, in which a very low level of 6-TGMP was also determined at T2. Serum levels of 5-ASA and N-Ac-5-ASA were 1,295 $(4-10,912)$ and 3,049 $(47-8,348)$ $\mathrm{ng} \cdot \mathrm{ml}^{-1}$. The 6-TGTP and 6-TGDP levels attributed $84 \%$ and $16 \%$ to the total $6-\mathrm{TGN}$ levels, comparable to T1 and T2. All except three patients had total 6-TGN levels above $235 \mathrm{pmol} / 8 \times 10^{8} \mathrm{RBC}$.

Compared with baseline (T1), an elevation of 6-TGDP and 6-TGTP metabolite levels was observed in 16 and 14 patients, respectively. The median absolute increase in total 6-TGN, 6-TGDP, 6-TGTP-levels at T3 was comparable to those found at T2, being 203, 36 and $164 \mathrm{pmol} / 8 \times 10^{8} \mathrm{RBC}$, respectively. Compared with $\mathrm{T} 2$, the median relative increase was marginal. Median 6-MMPR levels decreased significantly compared with T2 $(p=0.045)$ with a median decrease of $567 \mathrm{pmol} / 8 \times 10^{8} \mathrm{RBC}$. Again, no correlations were observed between 6-TGN, 6-TGDP and 6-TGTP or 6-MMPR levels and serum 5-ASA or $\mathrm{N}$-Ac-5-ASA levels at T3, nor was the increase in 6-TGN, 6-TGDP and 6-TGTP levels associated with 5-ASA or N-Ac-5-ASA serum levels.

\section{Metabolite levels at T4}

After discontinuation of 5-ASA (T4), the median levels of total 6-TGN ( $p=0.004), 6-T G T P$ $(p=0.006)$ and 6-TGDP ( $p=0.009)$ decreased significantly. Median levels (and range) of total 6-TGN, 6-TGTP, 6-TGDP and 6-MMPR were 449 (97-816), 381 (68-624), 69 (15-196) and 1,190 (0-9,811), respectively. Levels of 6-TGMP were undetectable in all patients, comparable to T1. Concentrations of 5-ASA and N-Ac-5-ASA could not be detected in the serum of any patient at T4. Of note, two patients showed total 6-TGN levels still higher than $235 \mathrm{pmol} / 8 \times 10^{8} \mathrm{RBC}$. Total 6-TGN levels consisted of 83\% 6-TGTP and $17 \%$ 6-TGDP levels, again comparable to all previous study phases. With respect to T3 the decline in median total 6-TGN levels by $123 \mathrm{pmol} / 8 \times 10^{8} \mathrm{RBC}$ was observed in 13 patients (77\%). For 6-TGDP and 6-TGTP, in 14 and 12 patients $(82 \%$ and $71 \%$, respectively) a median absolute decline of 19 and $102 \mathrm{pmol} / 8 \times 10^{8} \mathrm{RBC}$, respectively, was determined. Compared with T1, median metabolite levels of total 6 -TGN $(p=0.034)$ and 6-TGDP $(p=0.018)$ levels were still significantly higher. The relative difference was $13 \%$ and $30 \%$, representing 79 and $17 \mathrm{pmol} / 8 \times 10^{8}$ RBC. For 6-TGTP, the difference was $61 \mathrm{pmol} / 8 \times 10^{8} \mathrm{RBC}(+19 \%)$, but only a trend towards significance was calculated $(p=0.057)$. Median levels of $6-$ MMPR at T4 increased slightly by $310 \mathrm{pmol} / 8 \times 10^{8} \mathrm{RBC}$ when compared with $\mathrm{T} 3$, but were still lower at $486 \mathrm{pmol} / 8 \times 10^{8} \mathrm{RBC}$ when compared with T1. However, both changes were non-significant $(p=0.083$ and $p=0.78)$.

\section{Changes in 6-MMPR/6-TGN ratio}

A 6-MMPR/6-TGN ratio below 11 has been shown to correlate with clinical response to 6-MP therapy. ${ }^{7}$ In our study, the median 6-MMPR/6-TGN ratio was below this value at any time point (Figure 10.4). However, total 6 -TGN levels were always $>235 \mathrm{pmol} / 8 \times 10^{8}$ 
$\mathrm{RBC}$ in patients with a 6-MMPR/6-TGN ratio <11. At T1, the median 6-MMPR/6-TGN ratio was 4.7 (range: 0-36); a ratio below 11 was observed in 13 patients (60\%). Comedication with 2 g 5-ASA (T2) showed a non-significant decrease of the median 6-MMPR/6-TGN ratio towards 2.4 (range: $0-24 ; p=0.13$ ). A ratio below 11 was observed in 14 patients (82\%). Increasing 5-ASA dosage towards $4 \mathrm{~g}$ daily (T3) reduced the median 6-MMPR/6-TGN ratio further towards 1.7 (range: $0-11 ; p=0.032$ ) and a 6-MMPR/6-TGN ratio below 11 was observed in all patients at T3.

Discontinuation of 5-ASA at T4 resulted in a significant increase of the median 6-MMPR/6-TGN ratio to 2.9 (range: $0-14 ; p=0.01$ ) when compared with T3. A 6-MMPR/6-TGN ratio below 11 was still observed in 12 patients (71\%). No significant difference in median ratio was observed between T1 and T4 ( $p=0.5)$.

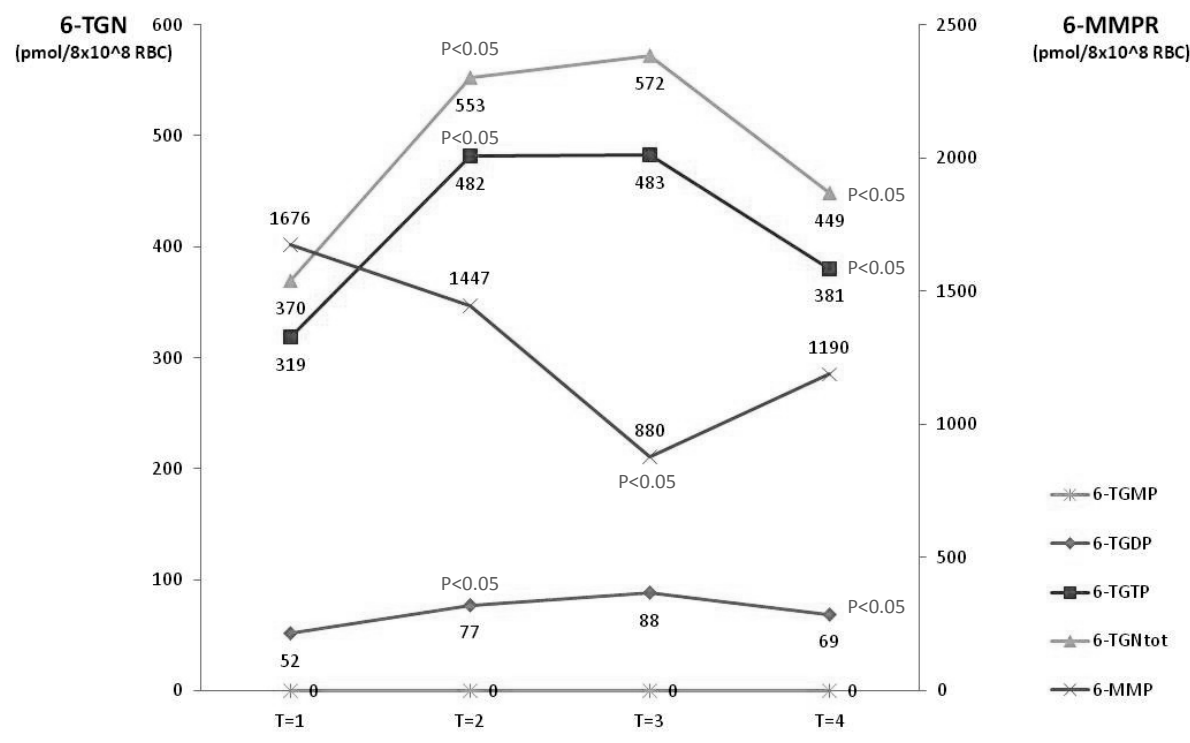

Figure 10.3 Influence of 5-ASA on individual thiopurine metabolite levels.

The influence of different doses of 5-ASA on the median thiopurine metabolite levels of 6-TGMP, 6-TGDP, 6-TGTP and 6-MMPR in patients on steady-state thiopurine therapy. T1, baseline, no 5-ASA; T2, 2 g 5-ASA daily; T3, 4 g 5-ASA daily; T4, washout, no 5-ASA; note that thiopurine doses were not altered over these study periods. 5-ASA, 5-aminosalicylate; 6-MMPR, 6-methylmercaptopurine ribonucleotides; 6-TGDP, 6-thioguanosine diphosphate; 6-TGMP, 6-thioguanosine monophosphate; 6-TGN, 6-thioguanine nucleotides; 6-TGTP, 6-thioguanosine triphosphate. 


\section{6-TGDP- and 6-TGTP-concentrations in relation to total 6-TGN-levels}

During the entire study period no alteration was seen for the relative contribution of 6-TGDP and 6-TGTP to total 6-TGN levels as calculated by the 6-TGTP/(6-TGTP+6-TGDP) ratio (Figure 10.4). Concerning 6-TGDP, the contribution was $14.5 \%$ at T1 (range: 9.6-28.8\%), $15.8 \%$ at T2 (range: $12.2-23.8 \%$ ), $15.7 \%$ at T3 (range: $12.1-32.4 \%$ ) and 17.1\% (range: $10.5-29.7 \%$ ) at T4. For 6-TGTP, the relative contribution to total 6-TGN was $85.5 \%$ at T1 (range: $71.2-90.4 \%$ ), $84.2 \%$ at T2 (range: $76.2-87.8 \%$ ), $84.3 \%$ at T3 (range: $67.6-87.9 \%$ ) and $82.9 \%$ (range: $70.3-89.5 \%$ ) at T4. In line with previous publications, ${ }^{25,26,27}$ the 6-TGMP levels were below or at the limit of detection. As the contribution of individual 6-TGN levels did not change, the median 6-TGTP/ (6-TGTP+6-TGDP) ratios were also comparable between all study periods: 0.86 at T1 (range: $0.71-0.90), 0.84$ at T2 (range: $0.76-0.88$ ), 0.84 at T3 (range: $0.68-0.88$ ) and 0.83 (range: $0.71-0.90$ ) at T4.

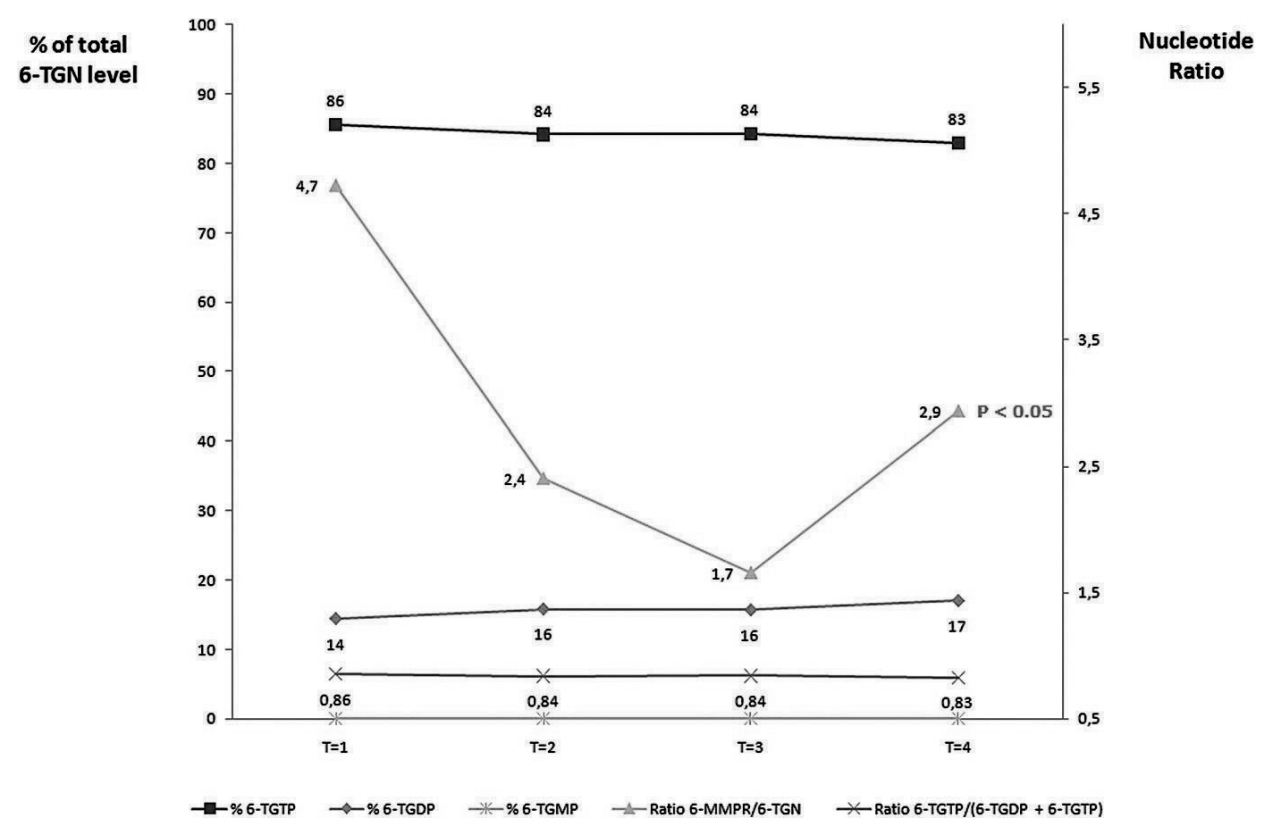

Figure 10.4 Influence of 5-ASA on different thiopurine metabolite ratios.

Contribution of the individual levels of 6-TGDP and 6-TGTP to total 6-TGN-levels and the changes in 6-MMPR/6-TGN-ratio under coadministration of different 5-ASA doses in patients under steady-state thiopurine therapy. T1, baseline, no 5-ASA; T2, 2 g 5-ASA daily; T3, 4 g 5-ASA daily; T4, washout, no 5-ASA; note that thiopurine doses were not altered over these study periods. 5-ASA, 5-aminosalicylate; 6-MMPR, 6-methylmercaptopurine ribonucleotides; 6-TGDP, 6-thioguanosine diphosphate; 6-TGMP, 6-thioguanosine monophosphate; 6-TGN, 6-thioguanine nucleotides; 6-TGTP, 6-thioguanosine triphosphate. 


\section{Discussion}

Increase of total 6-TGN levels after addition of 5-ASA has been reported before. ${ }^{12-18}$ However, in the present study we have demonstrated that the elevation of total 6-TGN levels is caused only by an increase in both 6-TGTP and 6-TGDP concentrations whereas 6-TGMP levels were under the LOQ. This is also reflected by a constant ratio of 6-TGTP/(6-TGTP+6-TGDP), not influenced by increasing doses of 5-ASA. The metabolite 6-TGTP is the main metabolite within the total pool of 6-TGN (approximately 85\%). Additionally, after dose escalation of 5-ASA to $4 \mathrm{~g}$ daily we have shown decreased 6-MMPR levels and subsequently an alteration of the 6-MMPR/6-TGN ratio.

As the metabolic pathway of thiopurines is complex, it is difficult to address the exact mechanism for this pharmacokinetic interaction. TPMT and xanthine oxidase (XO) are key enzymes in thiopurine metabolism, as both play an important role in the formation of the key metabolites, 6-TGN and 6-MMPR. Furthermore, both enzymes are also subject to pharmacogenetic polymorphisms. ${ }^{2}$

\section{The role of TPMT}

The TPMT-enzyme shunts thiopurine metabolism towards the formation of 6-MMPR after absorption (Figure 10.1). Therefore, inhibition of TPMT by 5-ASA could result in reduced 6-MMPR levels in favour of an increased formation of 6-TGN metabolites as we observed in our study. The effect of 5-ASA on TPMT-activity or thiopurine metabolite levels has been demonstrated in vitro and ex-vivo. ${ }^{12,14}$ However, conflicting data are available as several in vivo studies do not confirm these results. ${ }^{13,19,25}$ This may be the result of analytical problems, such as untimely 5-ASA removal from the sample matrix during analysis. However, Xin et al. have recently reported in vitro inhibitory concentrations $\left(/ C_{50}\right.$-values) values of 5-ASA $(129-236 \mu \mathrm{M})$ and N-Ac-5-ASA $(58-74 \mu \mathrm{M})$ for TPMT activity. ${ }^{14}$ Although in our study the median 5-ASA and N-Ac-5-ASA serum trough concentrations were below the published $I_{50}$-values for TPMT, our findings are in line with the results reported by others. ${ }^{26}$ Moreover, we cannot exclude the possibility that the $\mathrm{IC}_{50}$-values could be achieved intracellular, or for instance in $\mathrm{RBC}$ by influencing the expression/function of efflux membrane transporters, such as $A B C$ transporters, thereby resulting in an increase of 6-TGN as observed at T2 and a decrease of 6-MMPR levels at T3.

\section{Effect of 5-ASA on 6-TGMP, 6-TGDP and 6-TGTP-levels}

Earlier, we had demonstrated a dose-dependent effect of 5-ASA on total 6-TGN levels. ${ }^{18}$ From our current findings, it is clear that phosphorylation of 6-TGDP to 6-TGTP is not influenced by 5-ASA or N-Ac-5-ASA as no changes in 6-TGTP/(6-TGTP+6-TGDP) ratios were observed at all time points. This interaction therefore seems relevant for all 5-ASA formulations, including the new MMX extended release formulation (although this 
should be confirmed in clinical trials). Due to an increase in total 6-TGN and a decrease in 6-MMPR levels, the median 6-MMPR/6-TGN ratio decreased significantly. Of note, our present data clearly indicate that the increase in total 6-TGN levels is explained by the elevation of the individual 6-TGTP- and 6-TGDP levels. Furthermore, 6-TGTP remains the main metabolite within the total pool of 6-TGN (approximately 85\%) whereas the lack of 6-TGMP metabolites is in line with previous observations in IBD patients where individual 6-TGN levels were determined. ${ }^{23,27}$ Overall, our results show that determination of total 6-TGN levels will suffice for routine monitoring of thiopurine metabolites.

\section{Possible clinical importance of this pharmacological interaction}

Publications concerning clinical relevance of individual 6-thioguanosine phosphate levels are few, following the landmark study by Neurath et al.. ${ }^{23}$ They showed that higher 6-TGN levels combined with higher 6-TGDP levels were associated with reduced clinical response to AZA therapy. Furthermore, a 6-TGDP content of more than $15 \%$ of total 6-TGN levels was associated with poor response. Our study shows that the combination of AZA with $2 \mathrm{~g}$ 5-ASA daily will result in increased total 6-TGN as well as 6-TGTP levels while maintaining 6-TGDP levels around $15 \%$.

In addition, a reduction of 6-MMPR levels was observed after dose-escalation of 5-ASA to $4 \mathrm{~g}$ daily. Thus, theoretically an improved clinical response to AZA-therapy in IBD patients could be expected after co-administration with 5-ASA. Moreover, the combination of AZA and 5-ASA offers the opportunity to reduce the risk for hepatotoxic adverse reactions related to AZA, as elevated 6-MMPR levels have been clearly linked to hepatotoxicity. ${ }^{6,7}$ In consequence the compliance in taking AZA may also be improved. Shunting thiopurine metabolism from 6-MMPR towards 6-TGN has been suggested earlier and can probably be achieved by adding $5-\mathrm{ASA}^{13}$ and even possibly by allopurinol, a potent $\mathrm{XO}$ inhibitor, as recently mentioned. ${ }^{28,29}$

On the other hand, co-administration of 5-ASA could ultimately result in an increased risk for haematotoxicity such as leukopenia or bone marrow suppression. Signs of reversible myelotoxicity were observed in one patient of our study, possibly explained by a combined myelosuppressive effect of both 5-ASA and increased 6-TGN levels. Decreased TPMT activity based on genetic polymorphisms could be an additional risk factor for developing AZA-related myelosuppression, ${ }^{2,30}$ but unfortunately TPMT testing was not carried out in our trial.

Nevertheless, it should be mentioned that haematotoxicity can occur independent of TPMT activity in IBD patients treated with AZA, due to several other reasons, such as viral infection. ${ }^{31}$ Moreover, serious haematological adverse events will occur early in patients with intermediate or low TPMT activity after onset of AZA therapy. ${ }^{31,32}$ This is in line with the concept of a gene-dose effect related to TPMT. ${ }^{33}$ Thus even in IBD patients without TPMT testing, co-administration of 5-ASA with increasing doses might 
be a feasible approach. Nevertheless, regular laboratory controls (including haematological, hepatic and pancreatic markers), therapeutic drug monitoring and/or a prior-to-treat determination of TPMT activity may be helpful to predict a favourable risk/benefit ratio in the utilisation of thiopurines in IBD patients.

\section{Conclusion}

In conclusion, we have demonstrated in this study that coadministration of 5-ASA $2 \mathrm{~g}$ daily in patients on steady-state AZA therapy induces an increase in median 6-TGDP, 6-TGTP and total 6-TGN-levels. Subsequent increase of the 5-ASA dosage to $4 \mathrm{~g}$ daily showed no further elevation of individual 6-TGN levels in RBC, but a reduction in total 6-MMPR levels and a substantial decrease of the 6-MMPR/6-TGN ratio. Elevation of total 6-TGN levels was caused by an increase of both 6-TGTP and 6-TGDP metabolite levels. No alteration of 6-thioguanosine phosphate levels was found during treatment with 5-ASA. In theory, IBD patients unresponsive or refractory to standard thiopurine therapy may benefit from the coadministration of 5-ASA, leading to an increase of the pharmacologically active 6-TGN (i.e. 6-TGTP), a decrease in 6-MMPR levels and a reduced 6-MMPR/6-TGN-ratio. Monitoring of individual 6-TGN and 6-MMPR levels may provide detailed information concerning clinical response and treatment failure. To improve pharmacotherapy of thiopurines in treatment of patients with IBD, further clinical studies are warranted to elucidate our study results in representative patient cohorts. 


\section{References}

1. de Boer NK, van Bodegraven AA, Jharap B, de Graaf P, Mulder CJ. Drug Insight: pharmacology and toxicity of thiopurine therapy in patients with IBD. Nat Clin Pract Gastroenterol Hepatol 2007;4:686-94.

2. Teml A, Schaeffeler E, Herrlinger KR, Klotz U, Schwab M. Thiopurine treatment in inflammatory bowel disease: clinical pharmacology and implication of pharmacogenetically guided dosing. Clin Pharmacokinet 2007;46:187-208.

3. Tiede I, Fritz G, Strand S, Poppe D, Dvorsky R, Strand D, Lehr HA, Wirtz S, Becker C, Atreya R, Mudter J, Hildner K, Bartsch B, Holtmann M, Blumberg R, Walczak H, Iven H, Galle PR, Ahmadian MR, Neurath MF. CD28-dependent Rac1 activation is the molecular target of azathioprine in primary human CD4+ $T$ lymphocytes. J Clin Invest 2003;111:1133-45.

4. Poppe D, Tiede I, Fritz G, Becker C, Bartsch B, Wirtz S, Strand D, Tanaka S, Galle PR, Bustelo XR, Neurath MF. Azathioprine suppresses ezrin-radixin-moesin-dependent T cell-APC conjugation through inhibition of Vav guanosine exchange activity on Rac proteins. J Immunol 2006;176:640-51.

5. Osterman MT, Kundu R, Lichtenstein GR, Lewis JD. Association of 6-thioguanine nucleotide levels and inflammatory bowel disease activity: a meta-analysis. Gastroenterology 2006;130:1047-53.

6. Nygaard U, Toft N, Schmiegelow K. Methylated metabolites of 6-mercaptopurine are associated with hepatotoxicity. Clin Pharmacol Ther 2004;75:274-81.

7. Dubinsky MC, Yang H, Hassard PV, Seidman EG, Kam LY, Abreu MT, Targan SR, Vasiliauskas EA. 6-MP metabolite profiles provide a biochemical explanation for 6-MP resistance in patients with inflammatory bowel disease. Gastroenterology 2002;122:904-15.

8. Bokemeyer B, Teml A, Roggel C, Hartmann P, Fischer C, Schaeffeler E, Schwab M. Adherence to thiopurine treatment in out-patients with Crohn's disease. Aliment Pharmacol Ther 2007;26:217-25.

9. van Bodegraven AA, Mulder CJ. Indications for 5-aminosalicylate in inflammatory bowel disease: is the body of evidence complete? World J Gastroenterol 2006;12:6115-23.

10. Allgayer H, Ahnfelt NO, Kruis W, Klotz U, Frank-Holmberg K, Söderberg HN, Paumgartner G. Colonic $\mathrm{N}$-acetylation of 5-aminosalicylic acid in inflammatory bowel disease. Gastroenterology 1989;97:38-41.

11. Klotz U. Clinical pharmacokinetics of sulphasalazine, its metabolites and other prodrugs of 5-aminosalicylic acid. Clin Pharmacokinet 1985;10:285-302.

12. Szumlanski $\mathrm{CL}$, Weinshilboum RM. Sulphasalazine inhibition of thiopurine methyltransferase: possible mechanism for interaction with 6-mercaptopurine and azathioprine. Br J Clin Pharmacol 1995;39:456-9.

13. Dewit O, Vanheuverzwyn R, Desager JP, Horsmans Y. Interaction between azathioprine and aminosalicylates: an in vivo study in patients with Crohn's disease. Aliment Pharmacol Ther 2002;16: 79-85.

14. Xin H, Fischer C, Schwab M, Klotz U. Effects of aminosalicylates on thiopurine S-methyltransferase activity: an ex vivo study in patients with inflammatory bowel disease. Aliment Pharmacol Ther 2005;21:1105-9.

15. Hande S, Wilson-Rich N, Bousvaros A, Zholudev A, Maurer R, Banks P, Makrauer F, Reddy S, Burakoff R, Friedman S. 5-aminosalicylate therapy is associated with higher 6-thioguanine levels in adults and children with inflammatory bowel disease in remission on 6-mercaptopurine or azathioprine. Inflamm Bowel Dis 2006;12:251-7.

16. Lowry PW, Franklin CL, Weaver AL, Szumlanski CL, Mays DC, Loftus EV, Tremaine WJ, Lipsky JJ, Weinshilboum RM, Sandborn WJ. Leucopenia resulting from a drug interaction between azathioprine or 6-mercaptopurine and mesalamine, sulphasalazine, or balsalazide. Gut 2001;49:656-64.

17. Lewis LD, Benin A, Szumlanski CL, Otterness DM, Lennard L, Weinshilboum RM, Nierenberg DW. Olsalazine and 6-mercaptopurine-related bone marrow suppression: a possible drug-drug interaction. Clin Pharmacol Ther 1997;62:464-75.

18. de Boer NK, Wong DR, Jharap B, de Graaf P, Hooymans PM, Mulder CJ, Rijmen F, Engels LG, van Bodegraven AA. Dose-dependent influence of 5-aminosalicylates on thiopurine metabolism. Am J Gastroenterol 2007;102:2747-53.

19. Dilger K, Schaeffeler E, Lukas M, Strauch U, Herfarth H, Müller R, Schwab M. Monitoring of thiopurine methyltransferase activity in postsurgical patients with Crohn's disease during 1 year of treatment with azathioprine or mesalazine. Ther Drug Monit 2007;29:1-5. 
20 Derijks LJ, Gilissen LP, Engels LG, Bos LP, Bus PJ, Lohman JJ, Curvers WL, Van Deventer SJ, Hommes DW, Hooymans PM. Pharmacokinetics of 6-mercaptopurine in patients with inflammatory bowel disease: implications for therapy. Ther Drug Monit 2004;26:311-8.

21. de Graaf P, de Boer NK, Jharap B, Mulder CJ, van Bodegraven AA, Veldkamp Al. Stability of thiopurine metabolites: a potential analytical bias. Clin Chem 2008;54:216-8.

22. Karner S, Shi S, Fischer C, Schaeffeler E, Neurath MF, Herrlinger KR, Hofmann U, Schwab M. Determination of 6-thioguanosine diphosphate and triphosphate and nucleoside diphosphate kinase activity in erythrocytes: novel targets for thiopurine therapy? Ther Drug Monit 2010;32:119-28

23 Neurath MF, Kiesslich R, Teichgräber U, Fischer C, Hofmann U, Eichelbaum M, Galle PR, Schwab M. 6-thioguanosine diphosphate and triphosphate levels in red blood cells and response to azathioprine therapy in Crohn's disease. Clin Gastroenterol Hepatol 2005;3:1007-14.

24 Hussain FN, Ajjan RA, Moustafa M, Anderson JC, Riley SA. Simple method for the determination of 5aminosalicylic and $\mathrm{N}$-acetyl-5-aminosalicylic acid in rectal tissue biopsies. J Chromatogr B Biomed Sci Appl 1998;716:257-66.

25. Gilissen LP, Bierau J, Derijks L, Bos LP, Hooymans PM, van Gennip A, Stockbrügger RW, Engels LG. The pharmacokinetic effect of discontinuation of mesalazine on mercaptopurine metabolite levels in inflammatory bowel disease patients. Aliment Pharmacol Ther 2005;22:605-11.

26. Sandborn WJ, Hanauer SB. Systematic review: the pharmacokinetic profiles of oral mesalazine formulations and mesalazine pro-drugs used in the management of ulcerative colitis. Aliment Pharmacol Ther 2003;17:29-42.

27 Vikingsson S, Carlsson B, Almer SH, Peterson C Monitoring of thiopurine metabolites in patients with inflammatory bowel disease-what is actually measured? Ther Drug Monit 2009;31:345-50.

28. Sparrow MP, Hande SA, Friedman S, Cao D, Hanauer SB. Effect of allopurinol on clinical outcomes in inflammatory bowel disease nonresponders to azathioprine or 6-mercaptopurine. Clin Gastroenterol Hepatol 2007;5:209-14.

29 Ansari A, Elliott T, Baburajan B, Mayhead P, O'Donohue J, Chocair P, Sanderson J, Duley J. Long-term outcome of using allopurinol co-therapy as a strategy for overcoming thiopurine hepatotoxicity in treating inflammatory bowel disease. Aliment Pharmacol Ther 2008;28:734-41.

30 Schwab M, Schäffeler E, Marx C, Fischer C, Lang T, Behrens C, Gregor M, Eichelbaum M, Zanger UM, Kaskas BA. Azathioprine therapy and adverse drug reactions in patients with inflammatory bowel disease: impact of thiopurine S-methyltransferase polymorphism. Pharmacogenetics 2002;12:429-36.

31 Gisbert JP, Gomollon F. Thiopurine-induced myelotoxicity in patients with inflammatory bowel disease: a review. Am J Gastroenterol 2008;103:1783-800.

32 Colombel JF, Ferrari N, Debuysere H, Marteau P, Gendre JP, Bonaz B, et al. Genotypic analysis of thiopurine S-methyltransferase in patients with Crohn's disease and severe myelosuppression during azathioprine therapy. Gastroenterology 2000;118:1025-30.

33 Schaeffeler E, Fischer C, Brockmeier D, Wernet D, Moerike K, Eichelbaum M, Zanger UM, Schwab M. Comprehensive analysis of thiopurine S-methyltransferase phenotype-genotype correlation in a large population of German-Caucasians and identification of novel TPMT variants. Pharmacogenetics 2004;14:407-17. 


\section{Chapter 11}

The pharmacokinetic effect of adalimumab on thiopurine metabolism in Crohn's disease patients

DR Wong, M Pierik, ML Seinen, AA van Bodegraven, LPL Gilissen, P Bus, JA Bakker, AAM Masclee, C Neef, LGJB Engels, PM Hooymans

J Crohns Colitis 2014;8:120-128 


\section{Abstract}

Background and Aims

A drug interaction between infliximab and azathioprine has previously been reported in Crohn's disease patients: the concentration of the main active thiopurine metabolites, the 6-thioguanine nucleotides (6-TGN), increased 1-3 weeks after the first infliximab infusion with $50 \%$ compared to baseline.

The aim of this prospective study was to determine the effect of adalimumab on thiopurine metabolism in Crohn's disease patients, evaluated by 6-TGN and 6-methylmercaptopurine ribonucleotides (6-MMPR) concentration measurement.

\section{Methods}

Crohn's disease patients on azathioprine or mercaptopurine maintenance therapy starting with concomitant adalimumab treatment were included. 6-TGN and 6-MMPR concentrations were determined before initiation of adalimumab and after 2, 4, 6 and 12 weeks of combination therapy. The activity of three essential enzymes involving thiopurine metabolism, thiopurine S-methyltransferase (TPMT), hypoxanthine-guanine phosphoribosyltransferase (HGPRT) and inosine-triphosphate pyrophosphatase (ITPase), were evaluated at baseline and week 4. Clinical outcome was evaluated by the Crohn's disease activity index and C-reactive protein concentrations at baseline, week 4 and week 12 .

\section{Results}

Twelve Crohn's disease patients were analyzed. During the follow-up period of 12 weeks the median 6-TGN and 6-MMPR concentrations did not significantly change compared to baseline. TPMT, ITPase and HGPRT enzyme activity did not change either after 4 weeks. In two patients (17\%) myelotoxicity was observed within 2-4 weeks, in whom both low therapeutic 6-TGN concentrations were found.

\section{Conclusions}

In this study in Crohn's disease patients no pharmacokinetic interaction was shown between adalimumab and the conventional thiopurines, azathioprine and mercaptopurine. 


\section{Introduction}

The immunomodulating thiopurines, azathioprine (AZA) and mercaptopurine (6-MP), are effective for induction and particularly maintaining remission in the treatment of moderate to severe inflammatory bowel disease (IBD). Additionally, these drugs act as steroid-sparing agents. ${ }^{1,2,3}$

Neither AZA nor 6-MP has intrinsic pharmacological activity. AZA is a pro-drug that is converted to 6-MP by glutathion-S-transferase in the liver. 6-MP needs to undergo extensive metabolic transformations, involving pivotal enzymes like thiopurine S-methyltransferase (TPMT), xanthine oxidase (XO), hypoxanthine-guanine phosphoribosyl transferase (HGPRT) and inosine triphosphate pyrophosphatase (ITPase), yielding to a variety of pharmacological active metabolites. The thiopurine metabolites, 6-thioguanine nucleotides (6-TGN) and 6-methylmercaptopurine ribonucleotides (6-MMPR), are considered to be clinically the most important. The proposed thiopurine metabolism in human is given in Figure 11.1.

Patients with 6-TGN concentrations above the proposed therapeutic threshold of $235 \mathrm{pmol} / 8 \times 10^{8}$ red blood cells (RBC) are more likely to be in clinical remission than patients with a 6-TGN concentration below this threshold., ${ }^{5,6}$ Further, the risk for myelotoxicity increases with 6-TGN above $490 \mathrm{pmol} / 8 \times 10^{8} \mathrm{RBC}^{7,8}$ High concentrations of 6-MMPR (above $5,700 \mathrm{pmol} / 8 \times 10^{8} \mathrm{RBC}$ ) are associated with an increased risk of hepatotoxicity and treatment failure. ${ }^{8}$

In clinical practice therapeutic drug monitoring (TDM) of the thiopurine metabolites 6-TGN and 6-MMPR is a practical tool to optimise drug therapy in order to improve efficacy or avoid thiopurine toxicity. ${ }^{9,10}$

The biological drugs, infliximab and adalimumab, are monoclonal antibodies directed against tumour necrosis factor- $\alpha$ (TNF- $\alpha$ ), a pro-inflammatory cytokine produced by macrophages and activated T-cells, which plays a key role in the pathophysiology of inflammatory bowel diseases. Infliximab and adalimumab are effective for induction and maintenance of remission of Crohn's disease (CD) and ulcerative colitis (UC). ${ }^{11}$

According to national and international guidelines, anti-TNF- $\alpha$ treatment is indicated for steroid-refractory, steroid-dependent, or complex fistulising CD. ${ }^{11,12,13}$

In the SONIC-trial it was shown that patients with moderate-to-severe $C D$, who were treated with infliximab in combination with AZA, were more likely to have a corticosteroid-free clinical remission than those receiving AZA or infliximab monotherapy. ${ }^{14}$

In 2003, Roblin et al. described a pharmacokinetic interaction between infliximab and AZA in CD patients: 1-3 weeks after the first infliximab infusion the mean 6-TGN concentration increased in infliximab responders with 1.5 times the baseline level. Interestingly, the increase of 6-TGN concentration correlated with the clinical response to infliximab and AZA combination therapy. Three months after the first infliximab infusion, 6-TGN concentrations were comparable with the baseline-levels, which suggests a reversible effect of infliximab on AZA metabolism. ${ }^{15}$ Elevation of the active 
6-TGN, may result to an increased clinical efficacy, but may also increase the risk of severe myelotoxic side effects. ${ }^{8,16}$ Stringent safety monitoring is thus warranted.

The aim of this prospective study was to evaluate the effect of adalimumab on thiopurine pharmacokinetics in $C D$ patients being treated with stable thiopurine maintenance therapy.

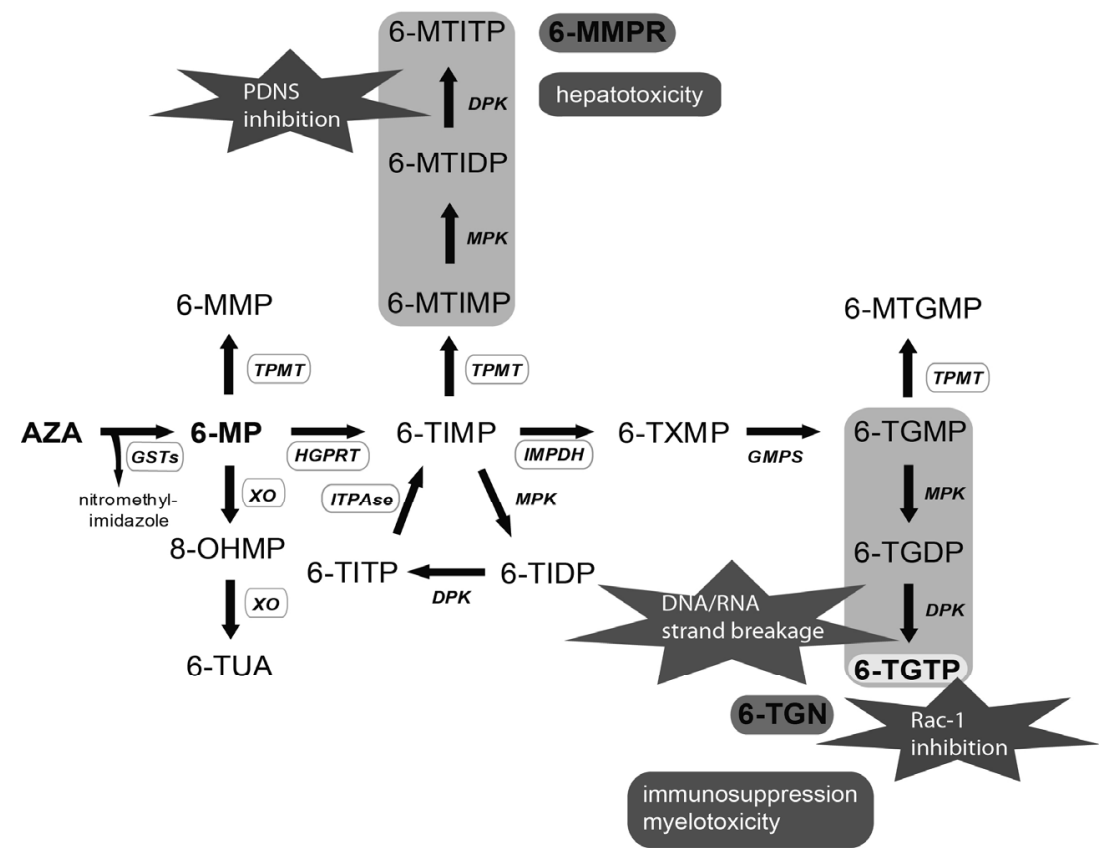

Figure 11.1 Proposed thiopurine metabolism.

AZA, azathioprine; 6-MP, 6-mercaptopurine; 6-MMP, 6-methylmercaptopurine; 8-OHMP, 8-hydroxy-6-mercaptopurine; 6-TUA, 6-thiouric acid; 6-MTIMP, 6-methylthioinosine monophosphate; 6-MTIDP, 6-methylthioinosine diphosphate; 6-MTITP, 6-methylthioinosine triphosphate; 6-TIMP, 6-thioinosine monophosphate; 6-TIDP, 6-thioinosine diphosphate; 6-TITP, 6-thioinosine triphosphate; 6-TXMP, 6-thioxanthosine monophosphate; 6-TGMP, 6-thioguanine monophosphate; 6-TGDP, 6-thioguanine diphosphate; 6-TGTP, 6-thioguanine triphosphate; 6-MTGMP, 6-methylthioguanine monophosphate; XO, xanthine oxidase; TPMT, thiopurine S-methyl transferase; HGPRT, hypoxanthine-guanine phosphoribosyl transferase; IMPDH, inosine monophosphate dehydrogenase; GMPS, guanosine monophosphate synthetase; MPK, monophosphate kinase; DPK, diphosphate kinase; ITPase, inosine triphosphate pyrophosphatase; PDNS, purine synthesis de novo; DNA, deoxyribonucleic acid; RNA, ribonucleic acid. 6-MTIMP, 6-MTIDP and 6-MTITP together form the 6-methylmercaptopurine ribonucleotides (6-MMPR). 6-TGMP, 6-TGDP and 6-TGTP form together the 6-thioguanine nucleotides (6-TGN). (Adapted from Derijks et al. ${ }^{4}$ ). 


\section{Materials and methods}

\section{Patient selection}

A prospective study was performed in a group of $C D$ patients under surveillance between July 2009 and December 2011 in five hospitals in the Netherlands (two university hospitals (Maastricht University Medical Centre and the VU University Medical Centre), and three general district hospitals (Orbis Medical Centre Sittard, Laurentius Hospital Roermond and Catharina Hospital Eindhoven)).

Steroid-dependent or steroid-refractory patients with ileo-colonic, colonic or peri-anal (fistulising) CD during maintenance AZA/6-MP therapy, who were scheduled for concomitant treatment with adalimumab, were prospectively included when meeting the following inclusion criteria: age between 18-70 years old, diagnosis of CD for at least 6 months ascertained by usual clinical, endoscopic and histological criteria, AZA/6-MP maintenance therapy for at least 3 months in stable dose for at least 6 weeks, normal renal function and no liver test abnormalities. Concomitant mesalazine therapy was registered and had to be stable for at least 8 consecutive weeks, because of the known interference with thiopurine metabolism in vivo. ${ }^{17}$

Patients were excluded from the study when one or more of the following criteria was met: signs of myelosuppression (leukocyte count $<2.5 \times 10^{9} / \mathrm{l}$ and/or platelet count $<100 \times 10^{9} / \mathrm{l}$ ), severe anaemia (haemoglobin $(\mathrm{Hb})$ concentration below $6 \mathrm{mmol} / \mathrm{l}$ ), known extensive proximal small bowel $C D$ possibly interfering with resorptive area, small bowel surgery leading to intestinal failure or short bowel syndrome, symptomatic ileal strictures or stenosis, concomitant use of allopurinol, angiotensin I-converting enzyme inhibitors, mycofenolate mofetil, infliximab or furosemide within 6 weeks before inclusion, concomitant use of methotrexate, current pregnancy or intention to become pregnant within 6 months before treatment or lactation.

\section{Study design}

Adalimumab therapy started with a subcutaneous induction dose of $160 \mathrm{mg}$ at week 0 and $80 \mathrm{mg}$ at week 2 , subsequently followed by a maintenance dose-regimen of $40 \mathrm{mg}$ subcutaneous every other week. Demographic and clinical data, concomitant medication and disease activity (Crohn's Disease Activity Index (CDAI)) were collected at baseline.

The study follow-up lasted for 12 weeks starting from the first adalimumab induction dose.

At baseline, week 2, 4, 6 and 12, blood samples were collected for 6-TGN and 6-MMPR metabolite assessment and evaluation of haematological and biochemical safety parameters. Adverse events were also recorded at these time points.

Clinical outcome was assessed at week 4 and 12 of concomitant adalimumab therapy, evaluated by CDAl calculation and C-reactive protein (CRP) serum concentration measurement. 
Written informed consent was obtained from all participating patients.

\section{Outcome parameters}

Primary objectives were changes in 6-TGN and 6-MMPR metabolite concentrations compared to baseline, resulting from concurrent adalimumab therapy. Secondary objectives were enzyme activity changes of three essential thiopurine metabolising enzymes, TPMT, ITPase and HGPRT, assessed before and 4 weeks after initiation of adalimumab therapy. Clinical response and safety were also evaluated during combination therapy.

Clinical remission was defined by a CDAl $<150$. In addition to CDAl evaluation, CRP serum concentrations were compared to baseline.

Safety was evaluated by measurement of the following haematological and biochemical parameters at baseline, weeks 2, 4, 6 and 12: haemoglobin count, leukocyte count, platelet count and erythrocyte count, mean corpuscular volume (MCV), haematocrit, CRP, aspartate aminotransaminase (ASAT), alanine aminotransaminase (ALAT), alkaline phosphatase (AP), gamma-glutamyl transferase (GGT), bilirubin, lactate dehydrogenase (LDH), albumin, amylase and creatinine.

\section{Analytical procedures}

\section{6-TGN and 6-MMPR concentrations}

Blood samples were immediately stored in the refrigerator $\left(2-8^{\circ} \mathrm{C}\right)$ and subsequently sent to the laboratory of the Department of Clinical Pharmacy \& Toxicology of the Orbis Medical Centre (Sittard-Geleen, The Netherlands), where the samples were processed and stored at $-20^{\circ} \mathrm{C}$ until required.

6-TGN and 6-MMPR metabolite concentrations were measured with a modified high performance liquid chromatography (HPLC) method of Lennard et al. as published previously. ${ }^{16}$ The lower-limits of quantification for 6-TGN and 6-MMPR were $40 \mathrm{pmol} / 8 \times 10^{8} \mathrm{RBC}$ and $300 \mathrm{pmol} / 8 \times 10^{8} \mathrm{RBC}$, respectively. The inter-assay variability for both thiopurine metabolites was less than $10 \%$.

\section{TPMT, HGPRT and ITPase enzyme activity}

TPMT enzyme activity was measured in erythrocyte lysates as described by JacquesAigrain using reversed phase HPLC for quantification. ${ }^{18}$

ITPase enzyme activity was measured in erythrocyte lysates by the production of inosine monophosphate (IMP) from inosine triphosphate. IMP was quantified using ionpairing HPLC with an external calibrant. ${ }^{19}$ 
HGPRT enzyme activity was measured by the production of IMP from hypoxanthine and phosphoribosyl pyrophosphate with a modified HPLC method of Jacomelli et al. using dried blood spot. IMP was quantified using ion-pairing HPLC with an external calibrant. $^{20}$

The enzyme activity measurements were performed at the Laboratory of Biochemical Genetics of the University Hospital Maastricht (Maastricht, The Netherlands). The interassay variation of each enzyme assay was less than $10 \%$.

\section{Statistical analysis}

Data are presented descriptively as the median and range. The data were compared using the non-parametric Wilcoxon test, since the outcome parameters did not show a normal distribution. Missing data were excluded from the relevant statistical analysis, when necessary. Correlation coefficients were calculated and significance of correlations were tested using Spearman's or Kendall's tau test, when appropriate. A p-value of less than 0.05 was considered to be statistically significant.

SPSS for windows version 17.0 was used for statistical analysis.

Power calculation of the sample size was based on a clinically relevant change of 6-TGN concentrations of more than $150 \mathrm{pmol} / 8 \times 10^{8} \mathrm{RBC}$ and a standard deviation of $75 \mathrm{pmol} / 8 \times 10^{8} \mathrm{RBC}$, according to a previous report from our group. ${ }^{16}$ Based on these data, at least 6 patients should be included to be able to demonstrate a statistically significant difference in 6-TGN levels with a probability of $80 \%$.

\section{Medical Ethics}

The study was initially approved by the local Medical Ethical Committee of Orbis Medical Centre, Sittard (The Netherlands) and subsequently by all other participating medical centres.

\section{Results}

\section{Patient demographics}

Thirteen patients ( 6 men, 7 women) with a median age of 24 years (range 18-46) were initially included.

Patient characteristics at baseline are given in Table 11.1. In two patients adalimumab dose was increased to $40 \mathrm{mg}$ every week because of a lack of response after 4 weeks and 10 weeks of treatment, respectively. The median daily AZA dose was $1.78 \mathrm{mg} / \mathrm{kg}$ (range 1.29-2.24; $\mathrm{n}=4$ ), and the median daily 6-MP dose was $0.77 \mathrm{mg} / \mathrm{kg}$ (range 0.27-1.23; $\mathrm{n}=9$ ). One patient required a reduced maintenance dose of 6-MP $(0.27 \mathrm{mg} / \mathrm{kg} /$ day) due to a previously revealed heterozygous TPMT genotype, assessed prior to thiopurine treatment. 
One patient treated with AZA, was excluded from data analysis due to non-compliance. Further analysis was performed with data from the remaining 12 patients.

\section{Thiopurine metabolite concentrations}

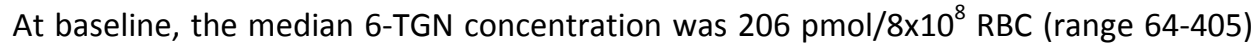

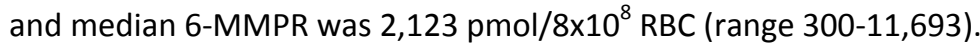
In the case of two patients the thiopurine blood sample of week 4 and week 6 were lost during shipping.

Table 11.1 Baseline patient characteristics.

\begin{tabular}{|c|c|}
\hline Patients & \\
\hline Patient number: $\mathrm{n}$ & 13 \\
\hline Median age in years (range) & $24(18-46)$ \\
\hline Gender (male / female) & $6 / 7$ \\
\hline CDAI (median) & 257 \\
\hline \multicolumn{2}{|l|}{ Crohn's disease } \\
\hline $\begin{array}{l}\text { Location }(\mathrm{n}=13 \text { ) } \\
\text { proctosigmoid } \\
\text { colon } \\
\text { ileal } \\
\text { ileo-colonic } \\
\text { perianal disease }\end{array}$ & $\begin{array}{l}2 \\
4 \\
5 \\
2 \\
1^{a}\end{array}$ \\
\hline $\begin{array}{l}\text { Behaviour ( } n=13 \text { ) } \\
\text { Inflammatory } \\
\text { Fistulising } \\
\text { Obstructive }\end{array}$ & $\begin{array}{l}12 \\
1 \\
2\end{array}$ \\
\hline Median disease duration in years (range) & $8(1-21)$ \\
\hline \multicolumn{2}{|l|}{ Thiopurine therapy } \\
\hline $\begin{array}{l}\text { Azathioprine }(\mathrm{n}) \\
\text { median AZA dose in } \mathrm{mg} / \mathrm{kg} \text { (range) }\end{array}$ & $\begin{array}{l}4 \\
1.78(1.29-2.24)\end{array}$ \\
\hline $\begin{array}{l}\text { 6-mercaptopurine }(\mathrm{n}) \\
\text { median 6-MP dose in } \mathrm{mg} / \mathrm{kg} \text { (range) }\end{array}$ & $\begin{array}{l}9 \\
0.77(0.27-1.23)\end{array}$ \\
\hline Median thiopurine exposure in months (range) & $48(3-149)$ \\
\hline \multicolumn{2}{|l|}{ Concomitant IBD medication at baseline } \\
\hline $\begin{array}{l}\text { Oral mesalazine } \\
\text { Rectal mesalazine }\end{array}$ & $\begin{array}{l}6 \\
1\end{array}$ \\
\hline $\begin{array}{l}\text { Oral steroids (i.e. predniso(lo)ne) } \\
\text { Topical steroids }\end{array}$ & $\begin{array}{l}7 \\
5\end{array}$ \\
\hline
\end{tabular}

\footnotetext{
${ }^{a}$ this patient suffered also from ileal Crohn's disease.
}

Figure 11.2 shows the individual 6-TGN and 6-MMPR concentration curves in time, based on the levels assessed at baseline and weeks 2, 4, 6 and 12. Overall, 6-TGN and 
6-MMPR metabolite concentrations did not significantly change during the first twelve weeks of concomitant adalimumab and thiopurine therapy (Table 11.2, Figure 11.3).

Before adalimumab therapy, in 8 of 13 patients (62\%) 6-TGN concentrations were found to be below the therapeutic threshold of $235 \mathrm{pmol} / 8 \times 10^{8} \mathrm{RBC}$. In 7 of 11 patients (64\%), who completed the study follow-up period, still suboptimal 6-TGN

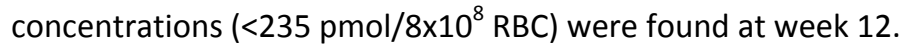

\section{TPMT, ITPase and HGPRT enzyme activity}

TPMT and ITPase enzyme activity were measured in 11 of 12 analysed patients, since the baseline sample of one patient was lost during shipping. HGPRT enzyme activity was measured in 8 of 12 patients at baseline and week 4, because three samples of the remaining patients were not appropriate for HGPRT enzyme activity analysis due to logistic problems.
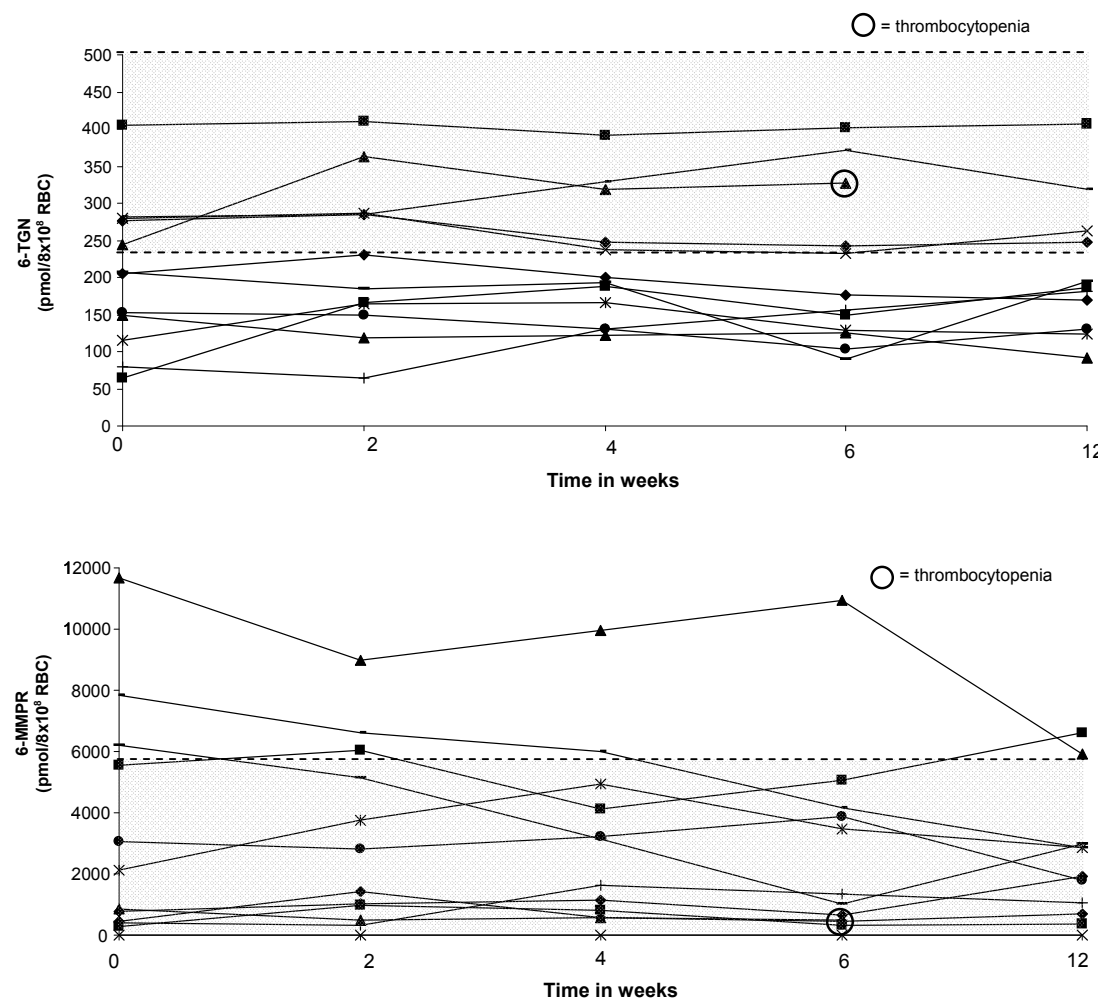

Figure 11.2 Individual 6-TGN level and 6-MMPR concentration curves until 12 weeks after initiation adalimumab therapy. 


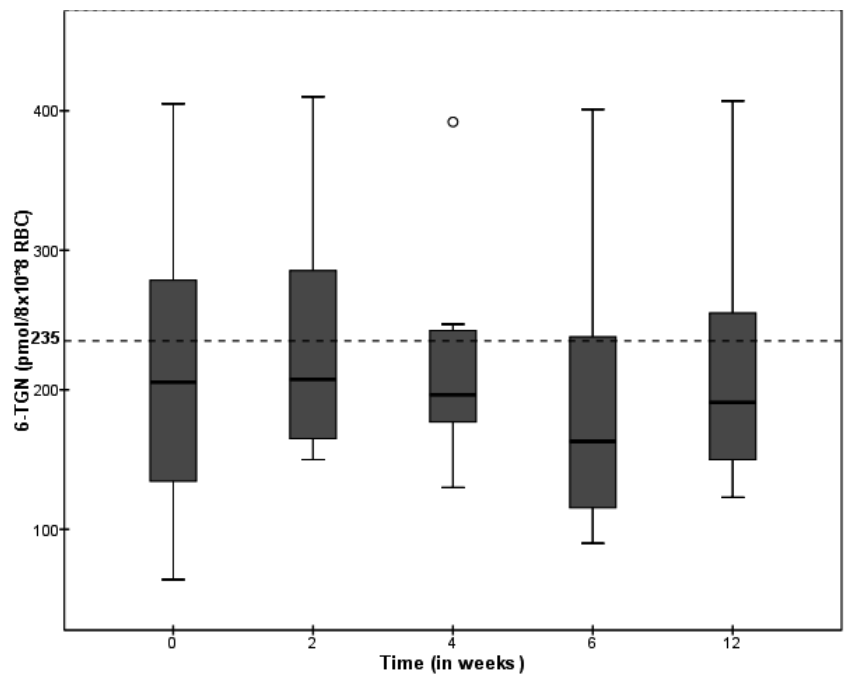

Figure 11.3 6-TGN concentration at baseline and 2, 4, 6 and 12 weeks after initiation of concurrent adalimumab therapy.

Table 11.2 Thiopurine metabolite levels at baseline (week 0) and 2, 4, 6 and 12 weeks after initiation of adalimumab therapy.

\begin{tabular}{|c|c|c|c|c|c|}
\hline & Week 0 & Week 2 & Week 4 & Week 6 & Week 12 \\
\hline $\begin{array}{l}\text { 6-TGN }{ }^{\mathrm{a}} \\
\left(\mathrm{pmol} / 8 \times 10^{8} \mathrm{RBC}\right)\end{array}$ & $\begin{array}{l}206 \\
(64-405)\end{array}$ & $\begin{array}{l}208 \\
(65-410)\end{array}$ & $\begin{array}{l}197 \\
(130-392)\end{array}$ & $\begin{array}{l}177 \\
(90-401)\end{array}$ & $\begin{array}{l}187 \\
(92-407)\end{array}$ \\
\hline $\begin{array}{l}\text { Difference compared to week } 0 \\
\text { (p-value) }\end{array}$ & ---- & 0.239 & 0.507 & 0.722 & 0.894 \\
\hline $\begin{array}{l}\text { 6-MMPR } \\
\left(\mathrm{pmol} / 8 \times 10^{8} \mathrm{RBC}\right)\end{array}$ & $\begin{array}{l}2,123 \\
(300-11,693)\end{array}$ & $\begin{array}{l}2,831 \\
(323-8,978)\end{array}$ & $\begin{array}{l}1,643 \\
(581-4,932)\end{array}$ & $\begin{array}{l}2,249 \\
(313-10,939)\end{array}$ & $\begin{array}{l}2,377 \\
(374-5,930)\end{array}$ \\
\hline $\begin{array}{l}\text { Difference compared to week } 0 \\
\text { (p-value) }\end{array}$ & ---- & 0.859 & 0.678 & 0.333 & 0.508 \\
\hline
\end{tabular}

${ }^{a}$ Median (range); 6-TGN and 6-MMPR were not normally distributed; $p$-value $<0.05$ was considered as statistically significant by comparison thiopurine levels of weeks 2, 4, 6 and 12 with baseline (week 0: $n=12$ ); weeks 4, 6 and 12: $n=11$.

Compared to baseline, TPMT, ITPase and HGPRT enzyme activity did not significantly change after 4 weeks of adalimumab and thiopurine co-treatment in the whole study group (Table 11.3).

In one patient an intermediate TPMT enzyme activity was found at baseline $(0.28 \mu \mathrm{mol}$ $6 \mathrm{MMP} /(\mathrm{mmol} \mathrm{Hb} \times \mathrm{h})$; ref. 0.45-0.67 $\mu \mathrm{mol} 6 \mathrm{MMP} /(\mathrm{mmol} \mathrm{Hb} \times \mathrm{h}))$. In another patient an intermediate ITPase enzyme activity was found $(0.03 \mathrm{mmol} \mathrm{IMP} /(\mathrm{mmol} \mathrm{Hb} \times \mathrm{h})$; ref. 3.00-10.00 mmol IMP/(mmol $\mathrm{Hb} \times \mathrm{h}))$. In all patients a normal HGPRT enzyme activity was measured (ref. 2.10-3.25 $\mathrm{mmol} \mathrm{IMP} /(\mathrm{mmol} \mathrm{Hb} \times \mathrm{h})$ ). 
Table 11.3 Median TPMT, ITPase and HGPRT enzyme activity at baseline and 4 weeks after adalimumab combination therapy.

\begin{tabular}{llll}
\hline & $\begin{array}{l}\text { TPMT } \\
(\mu \mathrm{mol} 6 \mathrm{MMP} / \\
(\mathrm{mmol} \mathrm{Hb} \times \mathrm{h})\end{array}$ & $\begin{array}{l}\text { ITPase } \\
(\mathrm{mmol} \mathrm{IMP/} \\
(\mathrm{mmol} \mathrm{Hb} \times \mathrm{h}))\end{array}$ & $\begin{array}{l}\text { HGPRT } \\
(\mathrm{mmol} \mathrm{IMP} / \\
(\mathrm{mmol} \mathrm{Hb} \times \mathrm{h}))\end{array}$ \\
\hline Baseline (week 0, range) & 0.54 & 3.96 & 2.55 \\
& $(0.28-0.78)$ & $(0.03-6.23)$ & $(2.25-3.24)$ \\
Week 4 (range) & 0.60 & 4.73 & 2.51 \\
& $(0.33-0.95)$ & $(0.01-11.3)$ & $(2.25-3.43)$ \\
Patient number $(\mathrm{n})$ & 11 & 11 & 8 \\
$\begin{array}{l}\text { Difference compared to week } 0 \\
\text { (p-value) }\end{array}$ & 0.142 & 0.142 & 0.799 \\
\hline
\end{tabular}

\section{Clinical outcome}

Before combination therapy, two of 12 patients (17\%) met the criteria of clinical remission (CDAI <150), but were treated with adalimumab because of steroiddependency. After 4 weeks of concurrent therapy, 7 of 12 patients (58\%) were in clinical remission. The median CDAI score at week 4 (127, range 67-292) significantly decreased compared to baseline (257, range 97-366; $p=0.004)$. Likewise, after 4 weeks the median CRP concentration (5, range 1-58) decreased compared to baseline (16, range $2.9-156, p=0.023$ ). After 12 weeks, $64 \%$ of the patients (7 of 11 ) were in clinical remission. Median CDAI score at week 12 (127, range 42-370) significantly decreased compared to baseline (257, range 97-366, $\mathrm{p}=0.008$ ). The median CRP concentration at week 12 (3.5, range 1-97) decreased compared to baseline (16, range 2.9-156, $p=0.047)$, but not compared to week $4(5$, range $1-58 ; p=0.796)$. There was no correlation between the clinical response rate, represented by the CDAI, and 6-TGN concentrations measured at weeks 4 and 12 ( $p=0.25$ resp. $p=0.401)$, and there was also no correlation between CRP and 6-TGN at these time points $(p=0.354$ and $p=0.272$, respectively).

\section{Laboratory safety parameters and adverse events}

During the entire study period no relevant elevated liver tests (ALAT, ASAT, AP, GGT, bilirubin) were observed in this study population. Parameters concerning pancreas (amylase) and renal function (creatinine) did not change either (data not shown). No alteration of MCV or erythrocyte count was observed. Compared to baseline, leukocyte count did not change at week 2, week 4, and week 6 . After 12 weeks of adalimumab therapy a decrease of the median leukocyte count was observed compared to baseline $\left(6.1 \times 10^{9} / /(4.0-10.6)\right.$ versus $\left.8.5 \times 10^{9} / /(4.3-10.6), p=0.011\right)$.

After 4 weeks one patient developed a temporary leukocytopenia with a leukocyte count of $3.5 \times 10^{9} /$ I (reference value: $4-10 \times 10^{9} / I$ ). Two weeks later the leukocyte count normalised without intervention. Another patient developed a thrombocytopenia after 
2 weeks, with a platelet count of $82 \times 10^{9} /$ / (reference value: $150-400 \times 10^{9} /$ I). Four weeks later platelet count dropped to $17 \times 10^{9} /$, whereupon 6-MP and adalimumab therapy were discontinued. Autoimmune thrombocytopenia was diagnosed, allegedly induced by adalimumab treatment. The patient was successfully treated with high-dose prednisolone. Overall, in the whole study group platelet count did not statistically significantly change during the follow-up period.

\section{Discussion}

In the present study in CD patients on stable maintenance thiopurine therapy, no significant effect of adalimumab was observed on 6-TGN and 6-MMPR metabolite concentration during the first 12 weeks of adalimumab and thiopurine combination therapy. Although after initiation of adalimumab therapy small individual changes in thiopurine metabolite concentrations were seen in several patients, we found no unequivocal overall effect in our study population, as opposed to the effect of infliximab on metabolism of AZA.

TPMT, ITPase and HGPRT enzyme activities did not change after 4 weeks of concomitant adalimumab therapy. Consequently, we believe that adalimumab neither induce nor inhibit these pivotal thiopurine metabolising enzymes in vivo.

Recent studies showed that AZA and infliximab combination therapy is more effective than when either is used as monotherapy, at least in naïve patients with moderate-tosevere active $C D$ and $U C .^{14,21,22}$ In line with this, results from a recent retrospective study may be interpreted that early combination of adalimumab and immunosuppressive therapy may lead to a decrease of flares and a lower risk of adalimumab failure. ${ }^{23}$ Combined anti-TNF- $\alpha$ and thiopurine therapy, started shortly after diagnosis, seems to have additional beneficial therapeutic effects in the treatment of active disease, although it is still debated for how long the combination should preferably be continued. ${ }^{14,21,23}$

The presented results concerning adalimumab in $C D$ patients treated with AZA or 6-MP, contrast with the earlier reported effect of infliximab on pharmacokinetics of AZA. ${ }^{15}$ An increase of 6-TGN concentration was shown within 1-3 weeks after the first infusion in $C D$ patients who received one (week $0 ; n=17$ ) or three infliximab infusions (week 0,2 and $6 ; n=15)$. Three months after the first infusion $6-$ TGN concentrations were similar to the concentrations before infliximab treatment in both patient groups. ${ }^{15} 6-\mathrm{MMPR}$ thiopurine metabolite concentrations were not reported and the pharmacological mechanism underlying this reversible pharmacokinetic interaction remained elusive. Since these patients received only one or three infusions and the follow-up was only the first three months, the clinical relevance of this interaction for the long-term clinical outcome is still unknown. We do not have an adequate explanation why infliximab affects 6-TGN formation and adalimumab does not, at least in our study cohort. However, in almost two-third of our patient group subtherapeutic 6-TGN 
concentrations were found, which is in contrast with the patients of the study population of Roblin et al. of whom in 90\% 6-TGN higher than $250 \mathrm{pmol} / 8 \times 10^{8} \mathrm{RBC}$ were found before infliximab infusion. ${ }^{15}$ Although unlikely, we can not exclude that addition of adalimumab to patients with higher or therapeutic thiopurine metabolites may lead to an increase of 6-TGN. Differences in anti-TNF- $\alpha$ plasma concentrations during infliximab and adalimumab therapy may also be an explanation, although this should be elucidated in future investigation. In a recent study of 81 patients performed by Teichgräber et al., a trend toward higher 6-TGN concentrations in patients on AZA and infliximab combination therapy did not reach statistical significance compared to patients on AZA monotherapy, which in fact is not in line with the findings of Roblin and colleagues. ${ }^{24}$ Interestingly, their results did reveal that infliximab co-treatment resulted in a higher 6-thioguanine triphosphate (6-TGTP) concentration, the 6-thioguanine nucleotide which in particular is considered to contribute to the immunosuppressive effect of thiopurines. ${ }^{24,25}$ In the present study only the sum of the 6-thioguanine nucleotides was assessed, so that the influence of adalimumab on the individual 6-thioguanine monophosphate, 6-thioguanine diphosphate and 6-thioguanine triphosphate formation remains elusive.

Addition of adalimumab to thiopurine monotherapy resulted in a decrease in leukocyte count at week 12 in our patients, which was also described 1-3 weeks after infliximab was added to AZA. ${ }^{15}$ Two patients (17\%) showed clinically relevant myelotoxicity within 2-4 weeks on combination therapy, which in one patient eventually lead to discontinuation of 6-MP and adalimumab, conceivably due to an autoimmune thrombocytopenia.

Myelotoxicity is one of the dose-dependent adverse events of thiopurines, mostly associated with elevated 6-TGN above $490 \mathrm{pmol} / 8 \times 10^{8} \mathrm{RBC}$. The reported rate of clinically relevant leukocytopenia in IBD patients varies between 2 and 11\%, depending on the definition of leukocytopenia. ${ }^{26}$ Haematologic adverse events are also frequently reported for adalimumab. Leukocytopenia occurs in more than $10 \%$ of patients treated with adalimumab and thrombocytopenia in $1-10 \% .{ }^{27}$ Since our patients developed myelotoxicity after addition of adalimumab and given the fact that in both patients low therapeutic 6-TGN concentrations were found, it indicates that combination therapy may lead to an increased risk of myelotoxicity, which is not attributable to elevated toxic 6-TGN, but rather to adalimumab. Alternatively, the myelotoxic effect of thiopurines may be potentiated by an additive myelotoxic effect of adalimumab, as has been documented before with concomitant use of mesalazine. ${ }^{17}$ Obviously, the observed decrease of leukocyte count at week 12 in the whole study population could also be attributed to a decrease of inflammatory disease activity resulting from combination therapy.

Our study has some limitations that should be mentioned. First, our study population of twelve patients is relatively small compared to previous relevant pharmacokinetic interaction studies on thiopurines. ${ }^{15,28,29}$ Second, there are some missing thiopurine metabolite concentration and enzyme activity data. Because of stability issues, strict 
storage and shipping time applied to these blood samples, we had to exclude the samples from analysis, as these were inappropriate for reliable measurement resulting from long shipping time. Third, international IBD guidelines advice standard daily dose regimen of AZA and 6-MP of 2-2.5 mg/kg and 1-1.5 mg/kg, respectively. ${ }^{10,13}$ The median daily thiopurine dose in our study was relatively low $(1.78 \mathrm{mg} \mathrm{AZA} / \mathrm{kg}$ and $0.77 \mathrm{mg}$ 6-MP/kg), which suggests suboptimal dosing. Patients treated with AZA or 6-MP who relapse or show poor response should be evaluated for adherence to therapy or underdosing. $^{6,10}$ In poor responding patients with low 6-TGN and low 6-MMPR concentrations due to underdosing, thiopurine dosage should be optimised before considering a change to methotrexate or costly anti-TNF- $\alpha$ therapy. ${ }^{10,13}$

In conclusion, this prospective study in CD patients does not provide evidence for a pharmacokinetic interaction between adalimumab and the conventional thiopurines AZA and 6-MP. Clinicians should be aware of an increased risk for myelotoxicity combining these immunosuppressive drugs. 


\section{References}

1. Prefontaine E, MacDonald JK, Sutherland LR. Azathioprine or 6-mercaptopurine for induction of remission in Crohn's disease. Cochrane Database of Systematic Reviews 2009: CD000545.

2. Prefontaine E, Sutherland LR, MacDonald JK, Cepoiu M. Azathioprine or 6-mercaptopurine for maintenance of remission in Crohn's disease. Cochrane Database of Systematic Reviews 2009:CD000067.

3. Timmer A, McDonald JW, MacDonald JK. Azathioprine and 6-mercaptopurine for maintenance of remission in ulcerative colitis. Cochrane Database of Systematic Reviews 2007:CD000478.

4. Derijks LJ, Wong DR. Pharmacogenetics of thiopurines in inflammatory bowel disease. Curr Pharm Des 2010;16:145-54.

5. Osterman MT, Kundu R, Lichtenstein GR and Lewis JD. Association of 6-thioguanine nucleotide levels and inflammatory bowel disease activity: a meta-analysis. Gastroenterology 2006; 130:1047-53.

6. Gilissen LP, Wong DR, Engels LG, Bierau J, Bakker JA, Paulussen AD, Romberg-Camps MJ, Stronkhorst A, Bus $P$, Bos LP, Hooymans PM, Stockbrügger RW, Neef C, Masclee AA. Therapeutic drug monitoring of thiopurine metabolites in adult thiopurine tolerant IBD patients on maintenance therapy. J Crohns Colitis 2012;6:698-707.

7. Cuffari C, Théorêt Y, Latour S, Seidman G. 6-Mercaptopurine metabolism in Crohn's disease: correlation with efficacy and toxicity. Gut 1996;39:401-6.

8. Dubinsky MC, Lamothe S, Yang HY, Targan SR, Sinnett D, Théorêt Y, Seidman EG. Pharmacogenomics and metabolite measurement for 6-mercaptopurine therapy in inflammatory bowel disease. Gastroenterology 2000;118:705-13.

9. Haines ML, Ajlouni Y, Irving PM, Sparrow MP, Rose R, Gearry RB, Gibson PR. Clinical usefulness of therapeutic drug monitoring in patients with inadequately controlled inflammatory bowel disease. Inflamm Bowel Dis 2011;17:1301-7.

10. Chouchana L, Narjoz C, Beaune P, Loriot MA, Roblin X. Review article: the benefits of pharmacogenetics for improving thiopurine therapy in inflammatory bowel disease. Aliment Pharmacol Ther 2012;35: 15-36.

11. D'Haens GR, Panaccione R, Higgins PD, Vermeire S, Gassull M, Chowers $Y$, Hanauer SB, Herfarth $H$, Hommes DW, Kamm M, Löfberg R, Quary A, Sands B, Sood A, Watermeyer G, Lashner B, Lémann M, Plevy S, Reinisch W, Schreiber S, Siegel C, Targan S, Watanabe M, Feagan B, Sandborn WJ, Colombel JF, Travis S. The London Position Statement of the World Congress of Gastroenterology on Biological Therapy for IBD with the European Crohn's and Colitis Organization: when to start, when to stop, which drug to choose, and how to predict response? Am J Gastroenterol. 2011;106:199-212; quiz 213. Review. Erratum in: Am J Gastroenterol 2011;106:375.

12. Van Bodegraven AA, Van Everdingen JJE, Dijkstra G, de Jong DJ, Oldenburg B en Hommes DW. Richtlijn 'Diagnostiek en behandeling van inflammatoire darmziekten bij volwassenen'. I. Diagnostiek en behandeling. Ned Tijdschr Geneeskd 2010;154:A1899.

13. Dignass A, Van Assche G, Lindsay JO, Lémann M, Söderholm J, Colombel JF, Danese S, D'Hoore A, Gassull M, Gomollón F, Hommes DW, Michetti P, O'Morain C, Oresland T, Windsor A, Stange EF, Travis SP; European Crohn's and Colitis Organisation (ECCO). The second European evidence-based Consensus on the diagnosis and management of Crohn's disease: Current management. J Crohns Colitis 2010;4: 28-62.

14. Colombel JF, Sandborn WJ, Reinisch W, Mantzaris GJ, Kornbluth A, Rachmilewitz D, Lichtiger S, D'Haens G, Diamond RH, Broussard DL, Tang KL, van der Woude CJ, Rutgeerts P; the SONIC Study Group. Infliximab, Azathioprine, or Combination Therapy for Crohn's Disease. N Engl J Med 2010;362:1383-95.

15. Roblin X, Serre-Debeauvais F, Phelip JM, Bessard G, Bonaz B. Drug interaction between infliximab and azathioprine in patients with Crohn's disease. Aliment Pharmacol Ther 2003; 18;917-925.

16. Derijks LJJ, Gilissen LPL, Engels LGJB, Bos LP, Bus PJ, Lohman JJHM, Curvers WL, Van Deventer SJH, Hommes DW, Hooymans PM. Pharmacokinetics of 6-mercaptopurine in patients with inflammatory bowel disease; implications for therapy. Ther Drug Monit 2004;26:311-8.

17. De Boer NK, Wong DR, Jharap B, de Graaf P, Hooymans PM, Mulder CJ, Rijmen F, Engels LG, van Bodegraven AA. Dose-dependent influence of 5-Aminosalicylates on thiopurine metabolism. Am J Gastroenterol 2007;102:1-7. 
18. Jacques-Aigrain E, Bessa E, Medard $\mathrm{Y}$, Mircheva $\mathrm{Y}$, Vilmer $\mathrm{E}$. Thiopurine methyltransferase activity in a French population: HPLC assay conditions and effects of drugs and inhibitors. Br J Clin Pharmacol 1994;38:1-8.

19. Bierau J, Bakker JA, Lindhout M, van Gennip AH. Determination of ITPase activity in erythrocyte lysates obtained for determination of TPMT activity. Nucleosides Nucleotides Nucleic Acids 2006;25:1129-32.

20. Jacomelli G, Micheli V, Peruzzi L, Notarantonio L, Cerboni B, Sestini S, Pompucci G. Simple nonradiochemical HPLC-linked method for screening for purine metabolism disorders using dried blood spot. Clin Chim Acta 2002;324:135-9.

21. Panaccione R, Ghosh S, Middleton S, Velazquez J, Khalif I, Flint L, van Hoogstraten HJ, Zheng H, Danese $S$, Rutgeerts $P$. Infliximab, azathioprine, or infliximab + azathioprine for treatment of moderate to severe ulcerative colitis: The UC SUCCESS trial. Oral presentation (Abstract OP 13): Presented at the European Crohn's and Colitis Organization (ECCO) congress, Stockholm 25 September 2011.

22. D'Haens G, Baert F, van Assche G, Caenepeel P, Vergauwe P, Tuynman H, De Vos M, van Deventer S, Stitt L, Donner A, Vermeire S, Van de Mierop FJ, Coche JC, van der Woude J, Ochsenkühn T, van Bodegraven AA, Van Hootegem PP, Lambrecht GL, Mana F, Rutgeerts P, Feagan BG, Hommes D; Belgian Inflammatory Bowel Disease Research Group; North-Holland Gut Club. Early combined immunosuppression or conventional management in patients with newly diagnosed Crohn's disease: an open randomised trial. Lancet 2008;371:660-7.

23. Reenaers C, Louis E, Belaiche J, Seidel L, Keshav S, Travis S. Does co-treatment with immunosuppressors improve outcome in patients with Crohn's disease treated with adalimumab? Aliment Pharmacol Ther 2012;36:1040-8.

24. Teichgräber U, Atreya I, Atreya R, Schwab M, Neurath MF. Infliximab treatment induces levels of the active azathioprine metabolite 6-TGTP in Crohn's disease. Inflamm Bowel Dis. 2013;19:E54-5.

25. Neurath MF, Kiesslich R, Teichgraber U, Fischer C, Hofmann U, Eichelbaum M, Galle PR, Schwab M. 6thioguanosine diphosphate and triphosphate levels in red blood cells and response to azathioprine therapy in Crohn's disease. Clin Gastroenterol Hepatol 2005;3:1007-14.

26. de Jong DJ, Derijks L, Naber AH, Hooymans PM, Mulder CJ. Safety of thiopurines in the treatment of inflammatorybowel disease. Scand J Gastroenterol Suppl 2003(239):69-72.

27. EMA. Summary of Product Characteristics: Humira , Abbott Laboratories Ltd, United Kingdom; [Internet]. Available at: http://www.emea.europa.eu/docs/nl_NL/document_library/EPAR_Product_ Information/human/000481/WC500050870.pdf. Accessed February 2nd, 2013.

28. Gilissen LP, Bierau J, Derijks LJ, Bos LP, Hooymans PM, van Gennip A, Stockbrügger RW, Engels LG. The pharmacokinetic effect of discontinuation of mesalazine on mercaptopurine metabolite levels in inflammatory bowel disease patients. Aliment Pharmacol Ther 2005;22:605-11.

29. de Graaf P, de Boer NK, Wong DR, Karner S, Jharap B, Hooymans PM, Veldkamp Al, Mulder CJ, van Bodegraven AA, Schwab M. Influence of 5-aminosalicylic acid on 6-thioguanosine phosphate metabolite levels: a prospective study in patients under steady thiopurine therapy. Br J Pharmacol 2010;160: 1083-91. 
Chapter 12

General discussion and future perspectives 
190 Chapter 12 


\section{General discussion and future perspectives}

The exact aetiology of IBD is multifactorial and still largely unknown. ${ }^{1}$ The main goal of treatment is to induce remission of disease, followed by therapy to maintain remission in order to achieve mucosal healing, reduce rates of hospital admission or avoid surgery. ${ }^{2-4}$

In the induction of remission of disease, aminosalicylates (5-ASA, mesalazine), budesonide and antibiotics are used in patients with mild disease, whereas systemic corticosteroids, immunomodulators and anti-tumor necrosis factor (TNF)- $\alpha$ agents are used in patients with moderate-to-severe IBD or in patients with mild disease not responding to standard medication. ${ }^{2,3}$ The immunomodulators include methotrexate, which is mainly effective in CD, and the thiopurines, azathioprine (AZA) and mercaptopurine (6MP). ${ }^{5-7}$

The monoclonal antibodies directed against the pro-inflammatory cytokine TNF- $\alpha$, are used to treat patients with moderate-to-severe IBD or who are refractory to conventional therapy. ${ }^{2,3}$ Anti-TNF agents have been shown to result in good efficacy and safety in UC and CD. . $^{8-15}$

Unfortunately, they are extremely costly and the long-term safety profile is not fully known. In addition, in view of the "loss of response" which may be seen in the course of time and the limited number of other effective drugs in IBD, it is important to optimise conventional IBD therapy. ${ }^{4}$

Thiopurines still are the mainstay of conventional maintenance therapy in IBD. In CD, induction of remission is achieved in at least half of patients receiving $A Z A$, while maintenance of remission is achieved in approximately $75 \%$ of the initial responders. ${ }^{5}$ In UC, according to a meta-analysis induction of clinical remission was reported in up to $78 \%$ of patients treated with thiopurines, while maintenance of clinical remission was observed in $60-75 \%$ of initial responders. ${ }^{16}$

In daily clinical practice the use of thiopurine immunosuppressants is limited due to occurrence of adverse drug events. ${ }^{17,18}$ Overall, the conventional thiopurine analogues AZA and 6MP lack efficacy in one-third of IBD patients. In addition, up to one-quarter of patients discontinue thiopurine therapy due to adverse reactions within the first months. $^{18,19}$

It has been shown that correctly optimised thiopurine treatment will safely maintain long-term remission in the majority of IBD patients. In this respect, correct dosing is essential and should become a target to improve prolonged efficacy and safety of thiopurine therapy.

Human thiopurine metabolism is complex because of the involvement of various metabolic enzymes resulting in large interindividual variations of active metabolite formation. $^{20,21}$ 
This thesis has presented new insights obtained from clinical studies on pharmacogenetics in thiopurine metabolism and assessment of 6-TGN and 6-MMPR metabolites in patients on thiopurine therapy, which have led to new strategies to improve efficacy and safety in the treatment of IBD.

\section{Main findings of the thesis}

- Despite the increasing knowledge on thiopurine metabolism, genetic polymorphisms of genes encoding for the crucial enzymes and their consequences, there is only limited information how to translate this knowledge into medical practice (Chapter 2).

- Thiopurine S-transferase (TPMT) genotype-based dosing results in a significant reduction of leukopenia in patients carrying a TPMT variant without impeding treatment efficacy (Chapter 3).

- TPMT genotype or a decreased TPMT enzyme activity cannot explain all cases of thiopurine-induced leukopenia, indicating that myelosuppression is often caused by other factors (Chapter 3).

- Xanthine oxidase is one of the most important enzymes involved in thiopurine metabolism, being responsible for large interindividual variations in active thiopurine metabolite formation. High xanthine oxidase enzyme activity may rarely even result in an inability to form active thiopurine metabolites (Chapter 4). Clinicians should be aware of this phenomenon when patients honestly declare to be thiopurine adherent.

- The previously reported therapeutic threshold of 6-TGN is confirmed in adult IBD patients to be $235 \mathrm{pmol} / 8 \times 10^{8} \mathrm{RBC}$. Thiopurine compliant IBD patients with steady-state 6-TGN concentrations above this therapeutic threshold are approximately 4 times more likely to have quiescent disease than patients with levels below the threshold (Chapter 5).

- Approximately one in six patients with IBD showed thiopurine non-compliance, both in patients with active and quiescent disease. Therapeutic drug monitoring of the active thiopurine metabolites is a practical clinical tool, which can be used in IBD patients on thiopurine maintenance therapy to reveal non-compliance or underdosing, and, especially in case of non-response, to optimise thiopurine therapy (Chapter 5).

- Both 6-TGN and 6-MMPR metabolites, assessed one week after initiation of thiopurine therapy (T1), are strongly correlated with the development of leukopenia during the first 8 weeks of therapy. In order to be able to predict the development of leukopenia, $T 1$ threshold concentrations of $213 \mathrm{pmol} / 8 \times 10^{8} \mathrm{red}$

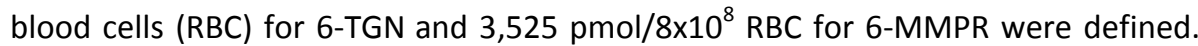
IBD patients exceeding either one of these values were approximately at a six-fold higher risk to develop leukopenia. Concomitant anti-TNF therapy demonstrated to be an additional independent risk factor for development of leukopenia in patients 
starting thiopurine therapy. A predictive algorithm was developed to identify patients at increased risk for thiopurine-induced leukopenia (Chapter 6).

- Hepatotoxicity, gastrointestinal complaints and general malaise are frequently occurring dose-dependent adverse reactions of thiopurines, related to elevated 6-MMPR metabolite concentrations. Patients exceeding the defined predictive threshold 6-MMPR concentration of $3,615 \mathrm{pmol} / 8 \times 10^{8} \mathrm{RBC}$ at week 1 were approximately at a 4-fold higher risk of hepatotoxicity during the first 20 weeks of treatment, and up to a 2.5 times higher risk to experience gastrointestinal complaints (i.e. nausea, vomiting and anorexia) and general malaise (Chapter 7).

- Thiopurine-induced hepatotoxicity strongly correlated with the male gender, higher age and body mass index (BMI). A predictive algorithm was developed to identify patients at risk of thiopurine-associated hepatotoxicity (Chapter 7).

- TPMT-deficient IBD patients can be successfully treated with a very low maintenance dose of the alternative thiopurine drug, 6-thioguanine, based on frequent metabolite testing to adjust and optimise thiopurine dose (Chapter 8 ).

- 5-aminosalicylates (5-ASA) influence the metabolism of thiopurines, which results in a dose-dependent increase of the active 6-TGN without an increase of the hepatotoxic 6-MMPR concentration (Chapter 9). Elevation of the total 6-TGN is caused by an individual increase of 6-thioguanine diphosphate (6-TGDP), but mainly 6-thioguanine triphosphate (6-TGTP) metabolite levels, which is considered to contribute to the immunosuppressive effects of the thiopurine drugs. IBD patients may benefit from the coadministration of 5-ASA, leading to an increase of the pharmacologically active 6-TGTP (Chapter 10).

- The results of a prospective interaction study in Crohn's disease patients did not provide evidence for a pharmacokinetic interaction between adalimumab and the conventional thiopurines AZA and 6MP (Chapter 11).

\section{Pharmacogenetics in thiopurine metabolism}

Thiopurine metabolism in human is complex yielding to a great variety of metabolites, as has been described in the Introduction (Chapter 1). The observed and reported interindividual differences in therapeutic response to and toxicity of thiopurines are to a large extent explained by the variable formation of active metabolites, which is at least partly caused by genetic polymorphisms of the genes encoding for the enzymes involving in thiopurine metabolism. In Chapter 2, pharmacogenetics of the crucial enzymes involving in thiopurine metabolism have been discussed.

In clinical practice, the dosages of thiopurines are based on bodyweight (AZA $(2.0-2.5 \mathrm{mg} / \mathrm{kg} /$ day $), 6 \mathrm{MP}(1.0-1.5 \mathrm{mg} / \mathrm{kg} / \mathrm{day})$ and $6 \mathrm{TG}(0.3 \mathrm{mg} / \mathrm{kg} / \mathrm{day}$, max. $25 \mathrm{mg} /$ day)), although there is no clear relation between the dose and thiopurine metabolite concentration due to large inter-patient variability. ${ }^{2,3,20-22}$ 
Glutathione S-transferase, xanthine oxidase and thiopurine S-methyltransferase enzymes are currently considered to be the most important enzymes contributing to the large interindividual variations in active metabolite formation (Chapter 2).

Polymorphisms in the gene encoding for glutathione S-transferase (i.e. M1-1) and the variable expression of GST (i.e. A1-1 and A2-2) imply differences in the rate of 6MP release from $A Z A$, influencing the drug activation and modulating oxidative stress and apoptosis affecting the risk of adverse reactions. ${ }^{23,24}$ Knowledge of genetic polymorphisms in genes for GSTs may be useful to predict response to AZA, but more in vitro and clinical validation studies are needed. ${ }^{25,26}$

However, these pharmacogenetic variations can easily be circumvented in clinical practice by using $6 \mathrm{MP}$ as such, rather than AZA. In fact, 6MP is the drug of choice for IBD in the United States of America, whereas in Europe, AZA is the only registered thiopurine for this indication.

Xanthine oxidase $(X O)$ is probably the most important enzyme determining thiopurine bioavailability. ${ }^{25}$ Interindividual differences in XO activity could be either genetically determined, or caused by environmental factors. ${ }^{27-29}$

Rapid XO metabolisers may fail to respond to thiopurine therapy due to low active metabolite formation or even the inability to generate detectable active thiopurine metabolites, as was demonstrated in the case of a woman with autoimmune hepatitis who was treated with a high dose of AZA, as described in Chapter 4. Although not explored, it is plausible that the high $\mathrm{XO}$ activity had a genetic basis. ${ }^{30}$ Clinicians should be aware of this rarely occurring phenomenon.

Interindividual differences in XO activity were demonstrated in a Japanese cohort, which were divided into low, normal and high enzyme activity. Two variants in the XO gene demonstrated to result in an increase of $X O$ enzyme activity. ${ }^{31}$ However, it is unclear to date whether these pharmacogenetic data can be extrapolated to Caucasian and/or other populations. At the moment, the added value of standard pharmacogenetic screening prior to treatment for genes expressing $\mathrm{XO}$ is questionable. More studies of larger populations are needed to evaluate the influence of $X O$ polymorphisms on clinical outcome and safety profile of thiopurine treatment of IBD. Since XO enzyme activity is influenced by environmental factors, including proinflammatory cytokines involved in chronic inflammatory diseases, it may contribute to a large extent to the interindividual, and perhaps intraindividual, variation of thiopurine metabolism.

Therefore, pharmacogenetics of xanthine oxidase and the relevant environmental factors influencing enzyme activity should become topics for future research on thiopurine therapy in IBD.

Thiopurine methyl-S-transferase enzyme determines the delicate balance between the formation of the immunosuppressive 6-TGN and the cytotoxic 6-MMPR metabolites. Genetic polymorphisms of TPMT have been extensively studied. Single nucleotide 
polymorphisms (SNPs) account for the major low TPMT activity forms, of which the TPMT $^{*} 3$ and TPMT ${ }^{*} 2$ mutant alleles are the most important and covering over $95 \%$ of the low activity alleles. Until now, over 35 variant TPMT alleles are reported. ${ }^{32}$

In Caucasians, the distribution of TPMT activity is trimodal, which can be related to genetic variants in the TPMT gene: $11 \%$ of the population is a heterozygous carrier of an inactive TPMT allele and $0.3 \%$ is homozygous for a TPMT variant, resulting in an intermediate and low or absent TPMT activity, respectively. Caucasians carrying two active alleles, approximately $89 \%$, show normal TPMT enzyme activity. Decreased TPMT enzyme activity shifts thiopurine metabolism towards increased formation of active cytotoxic 6-TGN metabolites. $^{33,34}$

Based on mainly retrospective studies, it has been shown that prior-to-treat TPMT testing helps to identify patients at risk of high accumulation of 6-TGN due to intermediate or absent TPMT enzyme activity. Patients with intermediate TPMT activity or carrying one TPMT variant allele were approximately four times more at risk of leukopenia during the early stage of treatment compared to patients with normal TPMT enzyme activity. $^{35,36}$

Since a large variation of TPMT activity among patients exists, some reports state that phenotype testing for practical purposes has a better predictive value for myelosuppression and allows for more accurate personalised dosing when compared to genotype. $^{37-40}$ of course, genotype-phenotype concordance can be improved by screening for greater numbers of TPMT variants. ${ }^{41}$

As presented in Chapter 3, a multicentre randomised-controlled trial (TOPIC) was performed in 769 IBD patients starting thiopurine therapy to evaluate whether pretreatment TPMT genotype-based thiopurine dosing reduces the risk of leukopenia compared to standard thiopurine dosing. Seventy-four patients $(9.6 \%)$ carried a TPMT variant $\left({ }^{*} 2,{ }^{*} 3 A\right.$ or $\left.{ }^{*} 3 C\right)$, and $58(7.5 \%)$ developed leukopenia (leukocyte count $<3.0 \times 10^{9} /$ I). Overall, the prevalence of leukopenia did not differ between the intervention and control group (7.2\% vs. $7.8 \%)$. However, compared to the control group, TPMT genotype-based dosing resulted in a significant reduction of leukopenia in patients carrying a TPMT variant in the intervention group (22.9\% versus $2.6 \%$, relative risk 0.11 (95\% $\mathrm{Cl}$ : 0.01-0.85). Steady-state 6-TGN and 6-MMPR assessed in patients of both groups were in the same range. ${ }^{42}$ In addition, prior-to-treat TPMT screening of IBD patients produced substantial cost-savings over the initial 20 weeks, without impeding treatment efficacy. ${ }^{43}$

The results of this study demonstrated that the TPMT genotype cannot explain all cases of leukopenia, a finding that is in accordance with previous reports. Thiopurine-induced leukopenia must therefore also be attributed to other factors besides a TPMT variant and a diminished TPMT enzyme activity, such as co-medication and/or viral infections. Since thiopurine-induced myelosuppression can occur at any time, and TPMT genotype or phenotype testing alone does not identify all patients at risk developing leukopenia, monitoring of full blood counts throughout the course of thiopurine treatment remains mandatory. ${ }^{44,45}$ 
Previous prospective studies have shown that TPMT phenotype assessment can be used to predict target doses and the likelihood of clinical response in patients with intermediate TPMT enzyme activity. ${ }^{46,47}$ Patients with an intermediate TPMT enzyme activity are unlikely to tolerate full doses of thiopurines and require $33-50 \%$ of the standard dose. ${ }^{17,46}$

IBD patients with homozygous TPMT variants and hence very low or even absent TPMT enzyme activity are generally recommended not to receive AZA or 6MP at all, because of the highly increased risk of serious thiopurine myelotoxicity when treated with a standard thiopurine dose. ${ }^{48}$ Chapter 8 describes the case of a patient with Crohn's disease who developed severe pancytopenia on a $50 \mathrm{mg} 6 \mathrm{MP}$ dose, which was associated with a high myelotoxic 6-TGN metabolite concentration resulting from complete TPMT enzyme deficiency. We demonstrated how this patient was successfully treated with a low dose of 6-thioguanine (6TG), which is suggested as an alternative thiopurine rescue drug in patients resistant or intolerant to conventional thiopurine therapy. $^{22}$ The 6TG dose was adjusted and optimised by means of frequent 6-TGN metabolite and complete blood cell count measurements, eventually resulting in a 6TG maintenance dose of $20 \mathrm{mg}$ every two weeks, approximately $7 \%$ of the standard dose. ${ }^{49}$ Four cases have previously been described demonstrating safe thiopurine therapy in homozygous TPMT mutant carriers by applying very low AZA dose ( 8-12\% of the standard AZA dose in IBD), guided by therapeutic drug monitoring. ${ }^{50,51}$ Recently an 18-year-old male Korean patient with Crohn's disease homozygous for TPMT*3C has been reported to be treated successfully on an AZA dose of $0.1-0.2 \mathrm{mg} / \mathrm{kg} / \mathrm{day}{ }^{52}$ However, great caution is warranted as a recent case demonstrated that even a very low AZA dose (5\% of standard) may result in myelotoxicity due to high 6-TGN concentrations. $^{53}$

These cases clearly demonstrate that TPMT deficiency not necessarily excludes thiopurine therapy. Thiopurine dosing should be carefully adjusted based on 6-TGN metabolite concentrations in a setting of intensive monitoring of metabolites and haematological parameters.

Despite the rapidly increasing knowledge on thiopurine metabolism, polymorphisms of genes encoding for the crucial enzymes and their consequences, only limited information exists on how to apply this knowledge to medical care. For example, in the Netherlands, the Pharmacogenetics Working Party has developed and implemented a national guideline with advices to apply TPMT pharmacogenetics in clinical practice in order to prevent thiopurine-induced myelosuppression in TPMT deficient patients. ${ }^{54}$

The translation of the currently existing knowledge into practical guidelines to be able to personalise thiopurine therapy will take much effort and costs. Future research is needed to evaluate the influence of genetic polymorphisms on clinical outcome of thiopurine treatment in IBD, and last but not least, how to implement this knowledge into clinical practice. 
Nevertheless, measurement of the most important active 6-TGN and 6-MMPR thiopurine metabolites reveals relevant insight of the 'ultimate phenotype' of all enzymes involving in the complex thiopurine metabolism in human.

\section{Therapeutic drug monitoring of thiopurine maintenance therapy in IBD}

\section{Therapeutic drug monitoring to optimise thiopurine efficacy}

The immunosuppressive effects of thiopurines are mainly attributed to the 6-TGN metabolites. ${ }^{55}$ In the clinical studies presented in this thesis, the thiopurine metabolites were assessed by a modified high-performance liquid chromatography (HPLC) method, which has been applied in the majority of clinical studies. ${ }^{20,56}$

The therapeutic range has been defined as a steady-state 6-TGN concentration between 235 and $490 \mathrm{pmol} / 8 \times 10^{8}$ red blood cells (RBC). ${ }^{35,57,58}$ A 6-MMPR/6-TGN ratio higher than 11 is correlated with a lower frequency of clinical response. ${ }^{20,59}$

Although most studies found no correlation between metabolite levels and thiopurine dose resulting from inter-individual differences in metabolism, in daily clinical practice thiopurine drugs are dosed based on the patient's bodyweight (AZA 2-2.5 mg/kg and 6MP $1-1.5 \mathrm{mg} / \mathrm{kg})^{2,3,48}$

Most of the data of studies on 6-TGN metabolite therapeutic thresholds have been obtained in small groups of IBD patients, in the early phase after initiating thiopurine therapy, in paediatric IBD cohorts and in some cases in retrospective studies. ${ }^{20,21,35,58-66}$ In the prospective cross-sectional study in adult IBD patients on thiopurine maintenance therapy, presented in Chapter 5, no significant difference of 6-TGN concentrations in patients with an exacerbation of disease was observed compared to patients in remission. However, the previous reported therapeutic 6-TGN cut-off level of $235 \mathrm{pmol} / 8 \times 10^{8} \mathrm{RBC}$ was confirmed in this study: 26 of the 41 patients (63\%) with active disease had 6-TGN levels below this threshold compared with 24 of 59 IBD patients $(41 \%)$ in clinical remission $(p=0.04){ }^{67}$

We found no correlation between 6-TGN levels, 6-MMPR/6-TGN ratio and disease activity, which is in line with conclusions from other reports on thiopurine metabolite testing. ${ }^{61,63-65}$

Thiopurine adherent IBD patients with steady-state 6-TGN concentrations above the therapeutic threshold were 3.8 times more likely to be in clinical remission. Our findings were in accordance with the results of a previously reported meta-analysis of six studies on the therapeutic 6-TGN threshold value showing that higher 6-TGN levels were correlated with clinical remission in both adults and children with IBD, with a pooled odds ratio of 3.3. ${ }^{66}$ A recent updated meta-analysis of ten studies using the reference HPLC method by Lennard et al. found a pooled Odds ratio (OR) of 3.15 for clinical remission among patients with 6-TGN levels exceeding a therapeutic cut-off 
level between 230 and $260 \mathrm{pmol} / 8 \times 10^{8} \mathrm{RBC}$. In the analysis of 17 studies enrolling 2,049 IBD patients over a 20-year period, an association between 6-TGN levels and clinical remission could be established: IBD patients with 6-TGN levels above the defined threshold were twice more likely to be in clinical remission. Important sources of heterogeneity of the latter analysis were the heterogeneity among the studies and the use of different HPLC reference methods. ${ }^{68}$

Based on the analysis of all patients in our study (Chapter 5), including the noncompliant patients, the defined therapeutic threshold 6 -TGN level of $235 \mathrm{pmol} / 8 \times 10^{8}$ showed rather poor sensitivity, specificity and positive predictive value of $72 \%, 60 \%$ and $52 \%$ respectively, resulting in a negative predictive value of $70 \%$ and an accuracy of $67 \%$, as has been reported in previous studies. ${ }^{66}$ The poor correlation between thiopurine dose and 6-TGN concentrations, indicates that individual metabolism of thiopurines is more important than the dose. ${ }^{66,67,69}$

Algorithms have recently been proposed to optimise therapy based on thiopurine metabolite testing. ${ }^{70-72}$

Up to now, the association between 6-TGN and clinical remission in IBD remains unclear. The study described in Chapter 5 was observational and not designed as interventional with dose adjustment based on thiopurine metabolite levels.

In order to improve the efficacy of thiopurine therapy, TDM with dose optimisation should be undertaken. Reinshagen et al. addressed this issue and concluded from a prospective study that an AZA dose-adjustment strategy based on therapeutic 6-TGN levels was not clinically superior to standard dosing with $2.5 \mathrm{mg} / \mathrm{kg}$ in patients with CD and normal TPMT activity. ${ }^{73}$ No significant differences concerning 6-TGN levels and disease activity were found between the standard and the intervention patient group at weeks 16 and 24. However, the study may have been underpowered $(n=57)$, which may explain why no significant results have been obtained. Gonzalez-Lama et al. tried to identify a therapeutic threshold for TPMT enzyme activity and 6-TGN concentration to predict safety and efficacy in steroid-resistant or -dependent IBD patients starting thiopurine therapy. No proper 6-TGN threshold value to adjust thiopurine dose could be identified. Early determination of TPMT activity and thiopurine metabolites proved not to be useful to predict clinical response and thiopurine safety. ${ }^{74}$ Once again, only a small number of patients has been evaluated and no significant differences in 6-TGN concentrations in responders and non-responders were observed.

A recent post-hoc analysis from a 1-year double-blind, randomised trial of 23 patients with moderate-to-severe post-operative recurrence of $C D$ reported for the first time on the correlation of thiopurine metabolites with mucosal healing. Only patients with an average concentration of $6-\mathrm{TGN}>193 \mathrm{pmol} / 8 \times 10^{8} \mathrm{RBC}$ had a significant improvement in their endoscopic score. These results provide first evidence on the utility of thiopurine metabolite testing to achieve mucosal response in $\mathrm{CD} .^{75}$

The role of thiopurine metabolite testing to optimise thiopurine therapy remains a matter of debate. In thiopurine-metabolite optimising strategies many confounding factors are involved, such as the large interindividual variation of thiopurine metabolite 
formation (also during therapy), the delayed onset of response after initiation (in general 2-4 months), the natural relapsing and remitting character of IBD, contributing to high placebo-response rates in clinical trials, thiopurine resistance among IBD patients and the fact that thiopurine metabolite levels in erythrocytes actually are a surrogate marker for the target cells, the leukocytes. ${ }^{21,25,70,76}$ In addition, the heterogeneity of IBD patient groups, which is often a mix of CD and UC patients, the use of different IBD activity scores and the effect of concurrent IBD medication, may be relevant factors as well.

Higher 6-TGN levels were found to be associated with clinical remission (Chapter 5). These data support the role of TDM in thiopurine maintenance therapy in IBD to reveal non-compliance or underdosing, and can be used as a practical tool to optimise thiopurine therapy, especially in case of thiopurine non-response. ${ }^{69,77-80}$

There is a need for larger long-term, prospective, randomised-controlled studies which follow patients on thiopurine treatment directly from the start of therapy, examining failure of treatment, efficacy and toxicity at several time points, comparing conventional follow up with dose optimisation guided by TDM.

\section{Therapeutic drug monitoring to reveal thiopurine non-compliance and underdosing}

The great interindividual variation in metabolism and the lack of a clear dose-response relation make the assessment of thiopurine metabolites useful to personalise thiopurine therapy. In Chapter 5 and 11, we demonstrated that the assessment of 6-TGN and 6-MMPR metabolites during thiopurine maintenance therapy reveals underdosing, but also nonadherence. ${ }^{67,81}$

An important finding of the study described in Chapter 5, was the relative high prevalence of nonadherence. In the population of one hundred IBD patients we studied, zero or very low thiopurine metabolite levels suggested non-compliance in $17 \%$ of all patients. In other words, approximately one in six patients was not adherent to thiopurine therapy, which was observed in patients with both active and quiescent disease. $^{67}$

Since medication nonadherence is associated with an increased rate of relapse, clinicians should be well aware that nonadherence may occur in up to $50 \%$ of the IBD patients. ${ }^{77,82-85}$ In daily practice, when patients on thiopurine maintenance therapy present with a flare of disease, frequently a switch to an alternative immunomodulator (i.e. methotrexate or anti-TNF therapy) is made. This has been demonstrated in the small study in CD patients starting with adalimumab: in two-thirds of patients subtherapeutic 6-TGN concentrations were found before anti-TNF therapy initiation, pointing to suboptimal thiopurine dosing (Chapter 11)..$^{81}$ In poorly responding patients with low 6-TGN and low 6-MMPR concentrations due to underdosing, thiopurine dosage should be optimised before considering a change to methotrexate or costly 
anti-TNF therapy. ${ }^{67,70}$ Given the limited therapeutic options to treat IBD, noncompliance or underdosing should first be excluded in these patients. ${ }^{81}$

Adherence behaviour of thiopurine therapy is hard to predict in clinical practice. Metabolite measurement is the only useful tool, especially when nonadherence is suspected, but denied by the patient. ${ }^{71,86}$ Once nonadherence is proven, patients should be supported and educated about the possible unfavourable consequences for their clinical course. Repeated metabolite testing may reveal insight whether the incentive approach works. In fact, since clinical data show that well optimised thiopurines will maintain long-term remission in the majority of IBD patients. Regular TDM of thiopurine therapy and frequent education may even help to prevent an exacerbation of disease.

It is important to note that very low or absent metabolite levels are not always related to non-adherence or underdosing. Rarely rapid XO metabolisers may be unable to generate detectable active thiopurine metabolites, as has been demonstrated in Chapter $4 .^{30}$ Clinicians should be aware of this phenomenon when patients honestly declare to be thiopurine adherent. In these cases, after non-compliance is ruled out, low-dose thiopurine maintenance therapy could be optimised in combination with allopurinol carefully directed by thiopurine metabolite testing and haematological monitoring. ${ }^{30,87-89}$

\section{Early assessment of Thiopurine metabolites to optimise safety in IBD}

\section{Early Thiopurine Metabolite assessment to predict leukopenia}

Thiopurines are associated with myelosuppression, most frequently leukopenia, which is considered to be the most important and potentially lethal adverse effect of thiopurine treatment in IBD patients. Leukopenia occurs most frequently during the first weeks to months after initiation of therapy in $2-5 \%$ of the patients. The risk of leukopenia increases for 6-TGN steady-state concentrations higher than $490 \mathrm{pmol} / 8 \times 10^{8} \mathrm{RBC}^{20,35,90}$ but extremely elevated 6-MMPR metabolites have also been associated with severe myelosuppression during thiopurine maintenance therapy. . $^{17,91,92}$

Steady-state thiopurine metabolite concentrations are generally reached after 4 weeks of treatment. ${ }^{20}$ Therefore, in clinical practice thiopurine metabolite assessment during steady-state is less useful to identify patients at risk to develop leukopenia during the first weeks of thiopurine treatment.

In Chapter 6, we reported on a prospective study on the predictive value of 6-TGN and 6-MMPR metabolites, assessed one week after initiation (T1), for the development of leukopenia during the first 8 weeks of treatment starting thiopurine therapy. ${ }^{93} T 1$ metabolite concentrations of 6-TGN and 6-MMPR were evaluated in patients with and 
without leukopenia ( $n=32$ and $n=162$, respectively). Both 6-TGN and 6-MMPR metabolite levels at $T 1$ were correlated with the occurrence of leukopenia: area under the Receiver Operating Characteristic (ROC) curves were $0.73(95 \% \mathrm{Cl}: 0.63-0.84$; $\mathrm{p}<0.0001)$ and $0.72(95 \% \mathrm{Cl}: 0.61-0.83 ; \mathrm{p}<0.0001)$, respectively. At the defined $T 1$ predictive threshold values of $213 \mathrm{pmol} / 8 \times 10^{8} \mathrm{RBC}$ for $6-\mathrm{TGN}$ and $3,525 \mathrm{pmol} / 8 \times 10^{8}$ RBC for 6-MMPR, patients with elevated T1 6-TGN or 6-MMPR levels were at increased risk of leukopenia during week 1-8: ORs were $6.2(95 \% \mathrm{Cl}: 2.8-13.8)$ and $5.9(95 \% \mathrm{Cl}$ : 2.7-13.3), respectively.

Until now, leukopenia has been mainly attributed to high cytotoxic 6-TGN concentrations, as a result of low TPMT enzyme activity or, in some cases, overdosing. Surprisingly, the 6-MMPR metabolites appeared also to relate to the occurrence of leukopenia showing a similar predictive value as the 6-TGN metabolites. Our study results showed that both 6-TGN and 6-MMPR are independently correlated with leukopenia and that the cytotoxic effect is presumably enhanced by one another. This may explain, at least for a part, the fact that thiopurine-induced leukopenia is not always related to low TPMT enzyme activity (Chapter 3 ).

Patients with leukopenia were more frequently treated with $6 \mathrm{MP}(1.0-1.5 \mathrm{mg} / \mathrm{kg} / \mathrm{day})$ than AZA (2-2.5 mg/kg/day) (Odds Ratio (OR)=7.3; 95\% Cl: 3.1-17.0). In addition, concurrent anti-TNF therapy at baseline (i.e. infliximab or adalimumab) was demonstrated to be an independent risk factor for the development of leukopenia in patients starting thiopurine therapy (OR $5.1 ; 95 \% \mathrm{Cl}: 1.6-16.4)$. Consequently, this finding indicates that the myelotoxic properties of thiopurines may be potentiated by additional myelotoxic effects of anti-TNF agents, as we previously reported on a small CD patient group showing mainly low-therapeutic 6-TGN concentrations and starting concomitant adalimumab therapy (Chapter 11). ${ }^{81}$ This observation is clinically relevant, since combined anti-TNF and thiopurine therapy seems to have beneficial therapeutic effects in the treatment of moderate-to-severe active inflammatory bowel disease and has been recommended to minimise the immunogenicity of anti-TNF monoclonal antibodies to prevent loss of response and infusion reactions. ${ }^{2,3,94-99}$

In order to predict the development of leukopenia in clinical practice a multivariable logistic regression model was developed based on the defined 6-TGN and 6-MMPR threshold values, thiopurine type and concomitant anti-TNF therapy. In our population the algorithm showed an accurate discriminatory power to predict leukopenia in clinical practice (AUC=0.84). ${ }^{100}$ Patients on concurrent anti-TNF therapy with elevations of both 6-TGN and 6-MMPR were demonstrated to be at highest risk to develop leukopenia. Patients on thiopurine monotherapy with both T1 6-TGN and 6-MMPR elevations were then at highest risk of developing leukopenia, followed by patients exceeding only the 6-MMPR or 6-TGN predictive threshold level at $T 1$.

Early identification of patients at increased risk of leukopenia is important as it may reduce morbidity, and to a (much) lesser extent hospitalisation or mortality. Therefore, in addition to pre-treatment TPMT genotyping, early assessment of 6-TGN and 6-MMPR 
metabolites at week 1 is a helpful tool to identify patients at increased risk of thiopurine-induced leukopenia.

\section{Early Thiopurine Metabolite assessment to predict hepatotoxicity, gastrointestinal complaints and general malaise}

Up to a quarter of IBD patients discontinue thiopurine treatment due to intolerable adverse events within the first 3 months of thiopurine therapy. ${ }^{18}$ Common adverse drug reactions prompting to discontinuation are gastrointestinal intolerance (i.e. nausea and vomiting) and hepatotoxicity. ${ }^{18,19}$

Thiopurine-induced hepatotoxicity occurs in up to $10 \%$ of patients, most frequently during the first months of therapy and often results to therapeutic failure. ${ }^{101-103}$

In a paediatric study, steady-state 6-MMPR metabolite concentrations exceeding $>5,700 \mathrm{pmol} / 8 \times 10^{8} \mathrm{RBC}$ have been associated with a 3 -fold increased risk of developing hepatotoxicity, which is in line with other reports in paediatric and adult patients. ${ }^{35,57,59,104}$ Thiopurine withdrawal due to hepatotoxicity has also been related to elevated steady-state 6-MMPR metabolite concentrations in adult IBD patients. ${ }^{18}$

The prospective study presented in Chapter 7 evaluated the predictive value of 6-MMPR metabolite concentrations, determined one week after thiopurine initiation (T1), for the development of thiopurine-induced hepatotoxicity, gastrointestinal complaints and general malaise. ${ }^{105}$

Forty-seven of 270 patients (17\%) presented signs of hepatotoxicity during the first 20 weeks of thiopurine treatment. 6-MMPR concentrations at week 1 were significantly higher in patients who developed hepatotoxicity and who reported gastrointestinal complaints (i.e. nausea, vomiting and/or anorexia) and/or general malaise. Patients exceeding the defined T1 6-MMPR threshold of $3,615 \mathrm{pmol} / 8 \times 10^{8} \mathrm{RBC}$ were at approximately 4-fold higher risk of hepatotoxicity (OR $3.8(95 \% \mathrm{Cl}: 1.8-8.0)$ ). The area under the ROC curve for the 6-MMPR concentration and hepatotoxicity in week 1-20 was $0.65(95 \% \mathrm{Cl}=0.55-0.75, \mathrm{p}=0.002)$, revealing a rather poor discriminative power. ${ }^{100}$ Previous studies have reported similar results, which underlines that hepatotoxicity also occurs in patients with low 6-MMPR concentrations and that thiopurine-associated hepatotoxicity may be caused by other factors. ${ }^{21,35,61,102,104}$

In our population, thiopurine-induced hepatotoxicity independently correlated with the male gender, age, bodyweight and body mass index (BMI) pointing to a combined influence of multiple factors. Age and BMI demonstrated the best discriminative value to predict hepatotoxicity in patients starting thiopurine therapy. Based on the most relevant determinants age, gender, BMI and the 6-MMPR threshold, we developed a multivariable regression model to predict hepatotoxicity with good accuracy $(\mathrm{AUC}=0.83)^{100}$

The predictive algorithm revealed that males, irrespective of age, with a BMI $>25 \mathrm{~kg} / \mathrm{m}^{2}$ and 6-MMPR concentrations at week 1 exceeding the defined threshold of $3,615 \mathrm{pmol} / 8 \times 10^{8} \mathrm{RBC}$, were at highest risk and all developed hepatotoxicity. Females 
meeting these criteria were then at highly increased risk of hepatotoxicity, followed by males or females older than 50 years with a BMI $>25 \mathrm{~kg} / \mathrm{m}^{2}$ with $T 1$ 6-MMPR concentrations below the defined threshold.

Interestingly, patients exceeding the $T 1$ 6-MMPR threshold of 6-MMPR $>3,615 \mathrm{pmol} / 8 \times 10^{8} \mathrm{RBC}$ were up to approximately 2.5 times more at risk to experience gastrointestinal complaints and general malaise. This is an important finding, as recently has been reported on a large long-term follow-up study that up to $80 \%$ of patients with thiopurine-induced nausea had to discontinue therapy. ${ }^{19}$ In our study, we found that patients who reported gastrointestinal complaints or general malaise were 2.5 to 3.5 times more likely to discontinue thiopurine treatment during the first five months.

Assessment of the 6-MMPR concentration one week after thiopurine initiation identifies patients at risk of hepatotoxicity, gastrointestinal complaints and general malaise and can be helpful to optimise thiopurine treatment in IBD to prevent these common adverse events frequently leading to thiopurine withdrawal.

Early Thiopurine Metabolite testing: a novel strategy to optimise thiopurine safety and tolerance in the future?

There are two important disadvantages of thiopurines that potentially limit the use of thiopurines in IBD. First, the intolerable adverse effects that mostly occur during the early stage of treatment. ${ }^{18,19,106}$ The second disadvantage is the delayed onset of the immunosuppressive effect, which can be expected after 2 to 4 months of therapy. Further, only up to $50-75 \%$ of the patients initially seem to benefit from thiopurine treatment, since a part of the patients discontinue during the course of therapy. ${ }^{18}$ Alternative therapeutic options are limited, therefore early identification of patients at risk of thiopurine limiting adverse events is important to improve thiopurine tolerance and effectiveness in IBD.

The two prospective studies presented in Chapter 6 and 7 demonstrated that the occurrence of the most common dose-dependent adverse reactions (i.e. leukopenia, hepatotoxicity, gastrointestinal complaints and general malaise) can be predicted based on the relevant risk factors and thiopurine metabolite measurements at week 1 , before steady-state concentrations are reached. ${ }^{20,93,105}$ Therefore, our findings are helpful to optimise thiopurine therapy in order to prevent unnecessary therapeutic failure, especially during the early stage of treatment.

The predictive threshold concentrations of 6-MMPR for thiopurine-induced leukopenia and hepatotoxicity, gastrointestinal intolerance and general malaise are by chance similar. In daily practice, a predictive $T 1$ 6-MMPR threshold concentration of $\sim 3,600 \mathrm{pmol} / 8 \times 10^{8} \mathrm{RBC}$ may be used to determine the risk of these adverse effects. The proposed 6-TGN and 6-MMPR threshold values and the predictive algorithms may be 
used to identify patients who are at increased risk of thiopurine-induced leukopenia and hepatotoxicity as early as one week after initiation. In these cases intensive haematological and liver test (LT) monitoring, early thiopurine dose reduction or a switch to alternative therapy may be warranted.

Assessment of the metabolite concentration at week 1 provides the clinician relevant information about the level of dosing, especially overdosing, when taking the defined 6-TGN therapeutic threshold of $235 \mathrm{pmol} / 8 \times 10^{8} \mathrm{RBC}$ and proposed therapeutic ranges at steady-state in account. $35,57,67,68$

In patients showing high 6-TGN and 6-MMPR concentrations at week 1 resulting from overdosing, thiopurine dose should be reduced. Patients showing elevated 6-TGN, but low 6-MMPR metabolite concentrations, presumably resulting from a diminished TPMT enzyme activity, thiopurine dosage should be lowered guided by haematological monitoring and metabolite assessment. Patients showing elevated 6-MMPR, but a relatively low 6-TGN concentration due to a preferential 6-MMPR metabolism (6-MMPR/6-TGN ratio $>20$ ), precautionary measures can be taken by reducing the thiopurine dose in combination with $100 \mathrm{mg} /$ day allopurinol. ${ }^{89,107-109}$ or by a switch to the alternative thiopurine 6-thioguanine $(0.3 \mathrm{mg} / \mathrm{kg} / \mathrm{day}) .{ }^{110-113}$

In patients who develop AZA-related hepatotoxicity or intolerance due to nausea, vomiting and flu-like symptoms, $6 \mathrm{MP}$ may be a good alternative, before definitely discarding thiopurine therapy. ${ }^{103,114}$ Also, a split-dose administration of AZA or 6MP has been reported to be an alternative option in patients with a preferential 6-MMPR metabolism and 6-MMPR associated side effects. ${ }^{115}$ As we have shown in Chapter 9 and 10 , addition of 5-ASA may be an attractive option to shift thiopurine metabolism towards the active 6-TGN guided by metabolite testing and safety monitoring. ${ }^{116,117}$ Especially in paediatric IBD patients this might be a safe option, given the limited efficacy and safety data on the use of 6TG and the coadministration of thiopurines and allopurinol in these patients.

Of course, the proposed predictive algorithms and thiopurine optimising considerations should be validated in future prospective randomised-controlled trials to evaluate whether the proposed strategies actually result into an improved efficacy and safety profile.

Patients with a skewed metabolism towards the 6-MMPR metabolites are more likely to fail to achieve adequate drug response due to subtherapeutic 6-TGN concentrations, resulting in a high 6-MMPR/6-TGN metabolite ratio (>20). Dose escalations result often in minor changes in 6-TGN, but major increases of the potentially toxic 6-MMPR, which frequently results in dose-dependent adverse effects and therapeutic failure. ${ }^{59,107}$

High TPMT enzyme activity appeared not to be the major reason for preferential 6-MMPR production in patients showing a high metabolite ratio. ${ }^{59,107}$

Recently, it has been demonstrated that a skewed metabolism leads to early therapeutic failure in the majority of IBD patients, mainly due to intolerance or refractoriness ( $70 \%) .{ }^{118}$ In $37 \%$ of the patients (101 of 270 ) we studied in Chapter 7 a 
high 6-MMPR/6-TGN metabolite ratio (>20) was found based on the metabolite levels assessed at week 1 . This indicates that patients with a skewed metabolism already may be identified after 1 week of therapy, which might be an interesting field for future research in order to reduce therapeutic failure.

The elimination half-life of 6-TGN and 6-MMPR metabolites is approximately 5 days, with a large variability of 3-13 days, at least for 6-TGN. Steady-state concentrations are reached after 25-30 days. ${ }^{20,119,120}$ The $T 1$ metabolite concentrations may provide information about the level of the concentration that will be reached at steady-state. Future prospective studies should be performed to elucidate the predictive value of the $T 1$ metabolite concentrations in this regard, but also on therapeutic response, which is interesting considering the delayed action of onset. Clinicians may then be able to adjust the dose before subtherapeutic or toxic steady-state concentrations have reached.

Thiopurine metabolism is complex and unpredictable due to the involvement of the various metabolic enzymes resulting in a great interindividual variation of pharmacological active thiopurine metabolite formation (Chapter 2). ${ }^{25}$ Therefore, assessment of thiopurine metabolites after one week of therapy is an attractive approach to reveal a patients 'ultimate phenotype'. In fact, the individual activity of all crucial enzymes involving in thiopurine metabolism (i.e. TPMT, HGPRT, XO, GST) is displayed by the proposed most important metabolites, the 6-TGN and 6-MMPR.

$T 1$ thiopurine metabolite testing offers new perspectives to improve thiopurine therapy by a more personalised pharmacological approach in the treatment of IBD.

\section{Pharmacokinetic interactions in thiopurine metabolism}

\section{5-Aminosalicylates and thiopurine therapy}

As described in Chapter 2, the activity of most enzymes is influenced by genetic and environmental factors, which may lead to an inhibition or induction of the enzymatic action. Some drugs have been reported to interfere in the thiopurine metabolic pathway. Several studies indicated that 5-aminosalicylic acid (5-ASA) compounds, such as mesalazine, olsalazine and sulphasalazine, may influence the metabolism of thiopurines, potentially leading to an increased risk of leukopenia due to the generation of elevated 6-TGN metabolites. ${ }^{121-126}$ Conclusions were restricted due to the number of patients or study design. ${ }^{122,125}$

The mechanism of this pharmacokinetic interaction remains to be elucidated, although several authors have suggested that TPMT activity is inhibited by sulphasalazine and other 5-ASA delivering drugs. ${ }^{121-123}$ 
In Chapter 9, a prospective pharmacokinetic study on the influence of 5-ASA on thiopurine metabolism in IBD patients during steady-state AZA or 6MP maintenance therapy has been described. ${ }^{116}$ We observed a dose-dependent increase of the 6-TGN concentrations after starting concurrent 5-ASA therapy: concomitant use of $2 \mathrm{~g}$ 5-ASA daily for four weeks resulted in a $40 \%$ increase of the median 6-TGN concentration (absolute $84 \mathrm{pmol} / 8 \times 10^{8} \mathrm{RBC}$ ), whereas addition of a daily dose of $4 \mathrm{~g} \mathrm{5-ASA}$ led to a $70 \%$ increase (absolute $154 \mathrm{pmol} / 8 \times 10^{8} \mathrm{RBC}$ ) compared to baseline.

In $8 \%$ of the patients signs of myelotoxicity were observed, which points to an increased risk of myelosuppression resulting from combination therapy, as previously was reported. ${ }^{64}$

The 6-thioguanine nucleotides consist of three different phosphorylated forms, namely 6-thioguanine monophosphate (6-TGMP), 6-thioguanine diphosphate (6-TGDP) and 6-thioguanine triphosphate (6-TGTP), as presented in Figure $1.1 .^{127}$ 6-TGTP, in particular, is considered to contribute to the immunosuppressive effects of the thiopurine drugs by inhibiting the function of the small GTPase Rac1 in activated lymphocytes leading to T-cell apoptosis. ${ }^{127,128}$

In the study described in Chapter 10, we evaluated the influence of 5-ASA on the individual 6-thioguanine phosphate metabolite concentrations in IBD patients $(n=17)$ on thiopurine maintenance therapy. ${ }^{117}$ We observed that coadministration of 5-ASA $2 \mathrm{~g}$ daily in patients on steady-state AZA therapy induced an increase in median 6-TGDP, 6-TGTP and total 6-TGN levels. A subsequent dose-escalation of the 5-ASA dosage to $4 \mathrm{~g}$ daily showed no further elevation of the individual 6-TGN levels in RBC, but an unexpected reduction in total 6-MMPR levels was observed. Elevation of total 6-TGN levels was caused by an increase of both 6-TGTP and 6-TGDP metabolite levels; 6-TGTP remained the main metabolite within the total pool of 6-TGN (approximately $85 \%$ of total 6-TGN).

In the study described in Chapter 9, overall the 6-MMPR concentrations were not affected, which indicates that the activity of the methylating TPMT enzyme was not influenced by 5-ASA or its metabolites in vivo, which is in contrast to several in vitro studies that demonstrated an inhibition of TPMT by 5-ASA compounds. The results of a prospective study showing that TPMT activity was not significantly affected by mesalazine monotherapy during a whole year of treatment were in line with our observations. ${ }^{129}$ These conflicting findings may be explained by analytical conditions, such as the untimely removal of 5-ASA from the target cell during in vitro analysis. A recent prospective study in 183 IBD patients showed overall no differences of TPMT enzyme activity, 6-TGN and 6-MMPR levels between patients with and without 5-ASA co-treatment. $^{130}$

On the other hand, in the study described in Chapter 10, we found that the 6-MMPR concentration decreased after a dose-escalation to $4 \mathrm{~g}$ 5-ASA in a subcohort of patients. In addition, no correlations were found between the rise of the (individual) 6-TGN, the 6-MMPR and serum 5-ASA or its metabolite N-Acetyl-5-ASA levels (Chapter 9 and 10). ${ }^{116,117}$ 
These findings emphasize the complexity of thiopurine metabolism and indicate that there may be more intracellular targets of interest besides TPMT, such as the possible influence on the function of the multidrug resistant efflux transporters MRP4 and MRP5 (Chapter 2). ${ }^{25}$

Current evidence regarding the pharmacokinetic interaction is strong, but the clinical relevance of 5-ASA and thiopurine combination is not yet proven. ${ }^{130,131}$ Two reports have shown that patients on AZA and 5-ASA with UC in remission may relapse severely when 5-ASA was withdrawn. ${ }^{132,133}$ The withdrawal of 5-ASA from patients on stable thiopurine therapy leads to a reduction of active 6-TGN metabolite levels, although it is not known whether this leads to reduced efficacy. ${ }^{116,125}$

The observed dose-dependent interaction may have important clinical implications in specific situations. IBD patients who are unresponsive or refractory to standard thiopurine therapy due to a preferential 6-MMPR metabolism often resulting in inadequate 6 -TGN levels ( $<235 \mathrm{pmol} / 8 \times 10^{8} \mathrm{RBC}$ ), may theoretically benefit from the coadministration of 5-ASA. In these patients combination therapy may lead to an increase of the pharmacologically active 6-TGN levels (i.e. 6-TGTP), without affecting or even decreasing the toxic 6-MMPR concentration. ${ }^{59,68,107}$ However, there is general evidence that concurrent therapy is associated with impaired drug adherence ${ }^{131}$ and clinicians should be aware of the increased risk of myelotoxicity and other limiting adverse effects (i.e. gastrointestinal complaints) when combining these drugs. ${ }^{64,134,135}$

Long-term prospective studies are needed to elucidate whether thiopurine and 5-ASA combination therapy results in an improvement of clinical outcome in thiopurine intolerant or resistant IBD patients due to inadequate thiopurine metabolite profiles.

\section{Anti-TNF agents and thiopurine therapy}

Infliximab is a monoclonal antibody against the pro-inflammatory cytokine tumor necrosis factor- $\alpha$ (TNF- $\alpha$ ) and has shown good efficacy in the induction and maintenance of remission in IBD. ${ }^{10,11}$ According to national and international guidelines, anti-TNF treatment is indicated for steroid-refractory, steroid-dependent, or complex fistulising CD. ${ }^{2,3,136,137}$

In 2003, Roblin et al. first described a pharmacokinetic interaction between infliximab and $A Z A$ in $C D$ patients. One to three weeks after the first infliximab infusion an increase in the mean 6-TGN concentration in infliximab responders was observed with $50 \%$ over the baseline level. Three months after the first infliximab infusion, 6-TGN concentrations were comparable with the baseline level, which suggests a reversible pharmacokinetic influence of infliximab on AZA metabolism. 6-MMPR thiopurine metabolite concentrations were not reported and the pharmacological mechanism underlying this reversible pharmacokinetic interaction remains elusive. ${ }^{138}$

Elevation of the active 6-TGN may result in an increased clinical efficacy, but may also increase the risk of severe myelotoxic side effects. ${ }^{20,35}$ 
The aim of the prospective study as described in Chapter 11 was to evaluate the effect of another anti-TNF agent (adalimumab) on thiopurine pharmacokinetics in twelve CD patients on stable AZA or 6MP maintenance therapy. During the follow-up period of 12 weeks the median 6-TGN and 6-MMPR concentrations did not significantly change compared to baseline. TPMT, ITPase and HGPRT enzyme activity did not change either after 4 weeks. Two patients (17\%) developed myelotoxicity within 2-4 weeks, in whom both low therapeutic 6-TGN and 6-MMPR concentrations were found.

The prospective study in CD patients did not provide evidence for a pharmacokinetic interaction between adalimumab and the conventional thiopurines. Our results are in contrast with the earlier reported effect of infliximab on the pharmacokinetics of AZA. ${ }^{138}$ Since these patients received only 1-3 infusions and the follow-up was only three months, the clinical relevance of this interaction for the long-term clinical outcome is still unknown.

We do not have an adequate explanation why infliximab affects 6-TGN formation and adalimumab does not, at least in our study. In a recent prospective study no significant differences were seen in either 6-TGN or in 6-MMPR or in TPMT enzyme activity between patients with and without concomitant treatment of AZA and infliximab. ${ }^{130}$ In another recent study, a trend towards higher 6-TGN concentrations in 81 patients on AZA and infliximab combination therapy did not reach statistical significance compared to patients on AZA monotherapy. ${ }^{139}$ In fact, the results of these studies are not in line with the previous findings of Roblin and colleagues. However, the latter study did reveal that infliximab co-treatment resulted in significant higher 6-TGTP concentrations. $^{139}$

Nevertheless, it has been proven that thiopurine and anti-TNF combination therapy is clinically more effective than either in monotherapy, at least in naïve patients with moderate-to-severe IBD. ${ }^{94-96}$ In addition, concomitant immunosuppression has been recommended to minimise the immunogenicity of anti-TNF monoclonal antibodies. ${ }^{98}$ Taking this in consideration, the combination of anti-TNF and immunomodulators has an important role in the treatment of IBD. Therefore, we believe that the clinical relevance of the pharmacokinetic influence on the (individual) 6-TGN metabolites of the older anti-TNF agents, but also the newer anti-TNF antibodies indicated to treat CD or UC, such as certolizumab pegol (off-label in the Netherlands) and golimumab, should be elucidated in further prospective clinical studies. 


\section{References}

1. Molodecky NA, Soon IS, Rabi DM, Ghali WA, Ferris M, Chernoff G, Benchimol El, Panaccione R, Ghosh S, Barkema HW, Kaplan GG. Increasing incidence and prevalence of the inflammatory bowel diseases with time, based on systematic review. Gastroenterology 2012;142:46-54 e42; quiz e30.

2. Dignass A, Lindsay JO, Sturm A, Windsor A, Colombel JF, Allez M, D'Haens G, D'Hoore A, Mantzaris G, Novacek G, Oresland T, Reinisch W, Sans M, Stange E, Vermeire S, Travis S, Van Assche G. Second European evidence-based consensus on the diagnosis and management of ulcerative colitis part 2: current management. J Crohns Colitis 2012;6:991-1030.

3. Dignass A, Van Assche G, Lindsay JO, Lémann M, Söderholm J, Colombel JF, Danese S, D'Hoore A, Gassull M, Gomollón F, Hommes DW, Michetti P, O'Morain C, Oresland T, Windsor A, Stange EF, Travis SP; European Crohn's and Colitis Organisation (ECCO). The second European evidence-based Consensus on the diagnosis and management of Crohn's disease: Current management. J Crohns Colitis 2010;4: 28-62.

4. Taylor KM, Irving PM. Optimization of conventional therapy in patients with IBD. Nat Rev Gastroenterol Hepatol 2011;8:646-56.

5. Prefontaine E, Macdonald JK, Sutherland LR. Azathioprine or 6-mercaptopurine for induction of remission in Crohn's disease. Cochrane Database Syst Rev 2009(4):CD000545.

6. Prefontaine E, Sutherland LR, Macdonald JK, Cepoiu M. Azathioprine or 6-mercaptopurine for maintenance of remission in Crohn's disease. Cochrane Database Syst Rev 2009(1):CD000067.

7. Timmer A, McDonald JW, Macdonald JK. Azathioprine and 6-mercaptopurine for maintenance of remission in ulcerative colitis. Cochrane Database Syst Rev 2007(1):CD000478.

8. Colombel JF, Sandborn WJ, Rutgeerts P, Enns R, Hanauer SB, Panaccione R, Schreiber S, Byczkowski D, Li $J$, Kent JD, Pollack PF. Adalimumab for maintenance of clinical response and remission in patients with Crohn's disease: the CHARM trial. Gastroenterology 2007;132:52-65.

9. Colombel JF, Sandborn WJ, Rutgeerts P, Kamm MA, Yu AP, Wu EQ, Pollack PF, Lomax KG, Chao J, Mulani PM. Comparison of two adalimumab treatment schedule strategies for moderate-to-severe Crohn's disease: results from the CHARM trial. Am J Gastroenterol 2009;104:1170-9.

10. Hanauer SB, Feagan BG, Lichtenstein GR, Mayer LF, Schreiber S, Colombel JF, Rachmilewitz D, Wolf DC, Olson A, Bao W, Rutgeerts P; ACCENT I Study Group. Maintenance infliximab for Crohn's disease: the ACCENT I randomised trial. Lancet 2002;359:1541-9.

11. Targan SR, Hanauer SB, van Deventer SJ, Mayer L, Present DH, Braakman T, DeWoody KL, Schaible TF, Rutgeerts PJ. A short-term study of chimeric monoclonal antibody CA2 to tumor necrosis factor alpha for Crohn's disease. Crohn's Disease cA2 Study Group. N Engl J Med 1997 9;337:1029-35.

12. Rutgeerts P, Sandborn WJ, Feagan BG, Reinisch W, Olson A, Johanns J, Travers S, Rachmilewitz D, Hanauer SB, Lichtenstein GR, de Villiers WJ, Present D, Sands BE, Colombel JF. Infliximab for induction and maintenance therapy for ulcerative colitis. N Engl J Med 2005;353:2462-76.

13. Sandborn WJ, Feagan BG2, Marano C3, Zhang H3, Strauss R3, Johanns J3, Adedokun OJ3, Guzzo C4, Colombel JF5, Reinisch W6, Gibson PR7, Collins J8, Järnerot G9, Hibi T10, Rutgeerts P11; PURSUIT-SC Study Group. Subcutaneous golimumab induces clinical response and remission in patients with moderate-to-severe ulcerative colitis. Gastroenterology 2014;146:85-95; quiz e14-5.

14. Sandborn WJ, Feagan BG, Marano C, Zhang H, Strauss R, Johanns J, Adedokun OJ, Guzzo C, Colombel JF, Reinisch W, Gibson PR, Collins J, Järnerot G, Rutgeerts P, PURSUIT-Maintenance Study Group. Subcutaneous golimumab maintains clinical response in patients with moderate-to-severe ulcerative colitis. Gastroenterology 2014;146:96-109 e1.

15. Sandborn WJ, van Assche G, Reinisch W, Colombel JF, D'Haens G, Wolf DC, Kron M, Tighe MB, Lazar A, Thakkar RB. Adalimumab induces and maintains clinical remission in patients with moderate-to-severe ulcerative colitis. Gastroenterology 2012;142:257-65 e1-3.

16. Gisbert JP, Linares PM, McNicholl AG, Mate J, Gomollon F. Meta-analysis: the efficacy of azathioprine and mercaptopurine in ulcerative colitis. Aliment Pharmacol Ther 2009;30:126-37.

17. Hindorf U, Lindqvist M, Peterson C, Söderkvist P, Ström M, Hjortswang H, Pousette A, Almer S. Pharmacogenetics during standardised initiation of thiopurine treatment in inflammatory bowel disease. Gut 2006;55:1423-31. 
18. Jharap B, Seinen ML, de Boer NK, van Ginkel JR, Linskens RK, Kneppelhout JC, Mulder CJ, van Bodegraven AA. Thiopurine therapy in inflammatory bowel disease patients: analyses of two 8-year intercept cohorts. Inflamm Bowel Dis 2010;16:1541-9.

19. Chaparro M, Ordás I, Cabré E, Garcia-Sanchez V, Bastida G, Peñalva M, Gomollón F, García-Planella E, Merino O, Gutiérrez A, Esteve M, Márquez L, Garcia-Sepulcre M, Hinojosa J, Vera I, Muñoz F, Mendoza JL, Cabriada JL, Montoro MA, Barreiro-de Acosta M, Ceña G, Saro C, Aldeguer X, Barrio J, Maté J, Gisbert JP. Safety of thiopurine therapy in inflammatory bowel disease: long-term follow-up study of 3931 patients. Inflamm Bowel Dis 2013;19:1404-10.

20. Derijks LJ, Gilissen LP, Engels LG, Bos LP, Bus PJ, Lohman JJ, Curvers WL, Van Deventer SJ, Hommes DW, Hooymans PM. Pharmacokinetics of 6-mercaptopurine in patients with inflammatory bowel disease: implications for therapy. Ther Drug Monit 2004;26:311-8.

21. Wright S, Sanders DS, Lobo AJ, Lennard L. Clinical significance of azathioprine active metabolite concentrations in inflammatory bowel disease. Gut 2004;53:1123-8.

22. de Boer NK, Reinisch W, Teml A, van Bodegraven AA, Schwab M, Lukas M, Ochsenkühn T, Petritsch W, Knoflach P, Almer S, van der Merwe SW, Herrlinger KR, Seiderer J, Vogelsang H, Mulder CJ; Dutch 6-TG working group. 6-Thioguanine treatment in inflammatory bowel disease: a critical appraisal by a European 6-TG working party. Digestion 2006;73:25-31.

23. Stocco G, Martelossi S, Barabino A, Decorti G, Bartoli F, Montico M, Gotti A, Ventura A. Glutathione-Stransferase genotypes and the adverse effects of azathioprine in young patients with inflammatory bowel disease. Inflamm Bowel Dis 2007;13:57-64.

24. Eklund BI, Moberg M, Bergquist J, Mannervik B. Divergent activities of human glutathione transferases in the bioactivation of azathioprine. Mol Pharmacol 2006;70:747-54.

25. Derijks LJ, Wong DR. Pharmacogenetics of thiopurines in inflammatory bowel disease. Curr Pharm Des 2010;16:145-54.

26. Stocco G, Pelin M, Franca R, De ludicibus S, Cuzzoni E, Favretto D, Martelossi S, Ventura A, Decorti G. Pharmacogenetics of azathioprine in inflammatory bowel disease: a role for glutathione-S-transferase? World J Gastroenterol 2014;20:3534-41.

27. Pacher $P$, Nivorozhkin A, Szabo $C$. Therapeutic effects of xanthine oxidase inhibitors: renaissance half a century after the discovery of allopurinol. Pharmacol Rev 2006;58:87-114.

28. Pritsos CA. Cellular distribution, metabolism and regulation of the xanthine oxidoreductase enzyme system. Chem Biol Interact 2000;129:195-208.

29. Zimm S, Collins JM, O'Neill D, Chabner BA, Poplack DG. Inhibition of first-pass metabolism in cancer chemotherapy: interaction of 6-mercaptopurine and allopurinol. Clin Pharmacol Ther 1983;34:810-7.

30. Wong DR, Derijks L, den Dulk MO, Gemmeke EH, Hooymans PM. The role of xanthine oxidase in thiopurine metabolism: a case report. Ther Drug Monit 2007;29:845-8.

31. Kudo M, Moteki T, Sasaki T, Konno Y, Ujiie S, Onose A, Mizugaki M, Ishikawa M, Hiratsuka M. Functional characterization of human xanthine oxidase allelic variants. Pharmacogenet Genomics 2008;18:243-51.

32. Lennard L. Implementation of TPMT testing. Br J Clin Pharmacol 2014;77:704-14.

33. Krynetski EY, Evans WE. Genetic polymorphism of thiopurine S-methyltransferase: molecular mechanisms and clinical importance. Pharmacology 2000;61:136-46.

34. Weinshilboum RM, Sladek SL. Mercaptopurine pharmacogenetics: monogenic inheritance of erythrocyte thiopurine methyltransferase activity. Am J Hum Genet 1980;32:651-62.

35. Dubinsky MC, Lamothe S, Yang HY, Targan SR, Sinnett D, Théorêt Y, Seidman EG. Pharmacogenomics and metabolite measurement for 6-mercaptopurine therapy in inflammatory bowel disease. Gastroenterology 2000;118:705-13.

36. Higgs JE, Payne K, Roberts C, Newman WG. Are patients with intermediate TPMT activity at increased risk of myelosuppression when taking thiopurine medications? Pharmacogenomics 2010;11:177-88.

37. Cooper SC, Ford LT, Berg JD, Lewis MJ. Ethnic variation of thiopurine S-methyltransferase activity: a large, prospective population study. Pharmacogenomics 2008;9:303-9.

38. Fangbin Z, Xiang G, Minhu C, Liang D, Feng X, Min H, Pinjin H Should thiopurine methyltransferase genotypes and phenotypes be measured before thiopurine therapy in patients with inflammatory bowel disease? Ther Drug Monit 2012;34:695-701.

39. Kwan LY, Devlin SM, Mirocha JM, Papadakis KA. Thiopurine methyltransferase activity combined with 6thioguanine metabolite levels predicts clinical response to thiopurines in patients with inflammatory bowel disease. Dig Liver Dis 2008;40:425-32. 
40. Winter JW, Gaffney D, Shapiro D, Spooner RJ, Marinaki AM, Sanderson JD, Mills PR. Assessment of thiopurine methyltransferase enzyme activity is superior to genotype in predicting myelosuppression following azathioprine therapy in patients with inflammatory bowel disease. Aliment Pharmacol Ther 2007;25:1069-77.

41. Booth RA, Ansari MT, Loit E, Tricco AC, Weeks L, Doucette S, Skidmore B, Sears M, Sy R, Karsh J. Assessment of thiopurine S-methyltransferase activity in patients prescribed thiopurines: a systematic review. Ann Intern Med 2011;154:814-23, W-295-8.

42. Coenen MJ, de Jong DJ, van Marrewijk CJ, Derijks LJ, Vermeulen SH, Wong DR, Klungel OH, Verbeek ALM,. Hooymans PM, Peters WHM, te Morsche RHM, Newman WG, Scheffer H, Guchelaar H-J, Franke B. Screening of the TPMT gene followed by personalized dosing of azathioprine and mercaptopurine treatment reduces the risk of leucopenia in patients with inflammatory bowel disease: a randomized trial. Submitted for publication 2014.

43. Coenen MJ van Marrewijk CJ, Derijks LJJ, Vermeulen SH, Wong DR, Klungel OH, Verbeek ALM, Hooymans PM, Scheffer H, Guchelaar H-J, Franke B, de Jong DJ. Personalized thiopurine dosing based on TPMT genotyping reduces leucopenia occurrence and results in cost-savings in IBD patients. Results from a randomized trial in the Netherlands. OP006. European Crohn's and Colitis Organization Congress; Copenhagen. 2014.

44. Colombel JF, Ferrari N, Debuysere H, Marteau P, Gendre JP, Bonaz B, Soulé JC, Modigliani R, Touze Y, Catala $\mathrm{P}$, Libersa C, Broly F. Genotypic analysis of thiopurine S-methyltransferase in patients with Crohn's disease and severe myelosuppression during azathioprine therapy. Gastroenterology 2000;118:1025-30.

45. Dewit O, Moreels T, Baert F, Peeters H, Reenaers C, de Vos M, Van Hootegem P, Muls V, Veereman G, Mana F, Van Outryve M, Holvoet J, Naegels S, Piessevaux H, Horsmans Y, Gala JL; Belgian Inflammatory Bowel Disease Research Group (BIRD). Limitations of extensive TPMT genotyping in the management of azathioprine-induced myelosuppression in IBD patients. Clin Biochem 2011;44:1062-6.

46. Gardiner SJ, Gearry RB, Begg EJ, Zhang M, Barclay ML. Thiopurine dose in intermediate and normal metabolizers of thiopurine methyltransferase may differ three-fold. Clin Gastroenterol Hepatol 2008;6:654-60; quiz 04.

47. Ansari A, Arenas M, Greenfield SM, Morris D, Lindsay J, Gilshenan K, Smith M, Lewis C, Marinaki A, Duley J, Sanderson J. Prospective evaluation of the pharmacogenetics of azathioprine in the treatment of inflammatory bowel disease. Aliment Pharmacol Ther 2008;28:973-83.

48. Sandborn WJ. Rational dosing of azathioprine and 6-mercaptopurine. Gut 2001;48:591-2.

49. Mares WG, Wong DR, Gilissen LP, Masclee AA, Hooymans PM, Engels LG. Safe 6-thioguanine therapy of a TPMT deficient Crohn's disease patient by using therapeutic drug monitoring. J Crohns Colitis 2009;3:128-30.

50. Kaskas BA, Louis E, Hindorf U, Schaeffeler E, Deflandre J, Graepler F, Schmiegelow K, Gregor M, Zanger UM, Eichelbaum M, Schwab M. Safe treatment of thiopurine S-methyltransferase deficient Crohn's disease patients with azathioprine. Gut 2003;52:140-2.

51. Gardiner SJ, Gearry RB, Barclay ML, Begg EJ. Two cases of thiopurine methyltransferase (TPMT) deficiency--a lucky save and a near miss with azathioprine. Br J Clin Pharmacol 2006;62:473-6.

52. Lee MN, Woo HI, Lee YM, Kang B, Kim JW, Choe YH, Lee SY. Successful azathioprine treatment with metabolite monitoring in a pediatric inflammatory bowel disease patient homozygous for TPMT*3C. Yonsei Med J 2013;54:1545-9.

53. Derijks L, van Helden RB, Hommes DW, Stokkers PC. Dosing azathioprine in thiopurine Smethyltransferase deficient inflammatory bowel disease patients. Gut 2008;57:872.

54. Swen JJ, Nijenhuis M, de Boer A, Grandia L, Maitland-van der Zee AH, Mulder H, Rongen GA, van Schaik RH, Schalekamp T, Touw DJ, van der Weide J, Wilffert B, Deneer VH, Guchelaar HJ. Pharmacogenetics: from bench to byte--an update of guidelines. Clin Pharmacol Ther 2011;89:662-73.

55. Lennard L. The clinical pharmacology of 6-mercaptopurine. Eur J Clin Pharmacol 1992;43:329-39.

56. Lennard L, Singleton HJ. High-performance liquid chromatographic assay of the methyl and nucleotide metabolites of 6-mercaptopurine: quantitation of red blood cell 6-thioguanine nucleotide, 6thioinosinic acid and 6-methylmercaptopurine metabolites in a single sample. J Chromatogr 1992;583:83-90.

57. Cuffari C, Theoret Y, Latour S, Seidman G. 6-Mercaptopurine metabolism in Crohn's disease: correlation with efficacy and toxicity. Gut 1996;39:401-6. 
58. Cuffari C, Hunt S, Bayless T. Utilisation of erythrocyte 6-thioguanine metabolite levels to optimise azathioprine therapy in patients with inflammatory bowel disease. Gut 2001;48:642-6.

59. Dubinsky MC, Yang H, Hassard PV, Seidman EG, Kam LY, Abreu MT, Targan SR, Vasiliauskas EA. 6-MP metabolite profiles provide a biochemical explanation for 6-MP resistance in patients with inflammatory bowel disease. Gastroenterology 2002;122:904-15.

60. Achkar JP, Stevens T, Easley K, Brzezinski A, Seidner D, Lashner B. Indicators of clinical response to treatment with six-mercaptopurine or azathioprine in patients with inflammatory bowel disease. Inflamm Bowel Dis 2004;10:339-45.

61. Goldenberg BA, Rawsthorne $\mathrm{P}$, Bernstein CN. The utility of 6-thioguanine metabolite levels in managing patients with inflammatory bowel disease. Am J Gastroenterol 2004;99:1744-8.

62. Hindorf $U$, Lyrenas E, Nilsson A, Schmiegelow K. Monitoring of long-term thiopurine therapy among adults with inflammatory bowel disease. Scand J Gastroenterol 2004;39:1105-12.

63. Belaiche J, Desager JP, Horsmans Y, Louis E. Therapeutic drug monitoring of azathioprine and 6mercaptopurine metabolites in Crohn disease. Scand J Gastroenterol 2001;36:71-6.

64. Lowry PW, Franklin CL, Weaver AL, Szumlanski CL, Mays DC, Loftus EV, Tremaine WJ, Lipsky JJ, Weinshilboum RM, Sandborn WJ. Leucopenia resulting from a drug interaction between azathioprine or 6-mercaptopurine and mesalamine, sulphasalazine, or balsalazide. Gut 2001;49:656-64.

65. Gupta P, Gokhale R, Kirschner BS. 6-mercaptopurine metabolite levels in children with inflammatory bowel disease. J Pediatr Gastroenterol Nutr 2001;33:450-4.

66. Osterman MT, Kundu R, Lichtenstein GR, Lewis JD. Association of 6-thioguanine nucleotide levels and inflammatory bowel disease activity: a meta-analysis. Gastroenterology 2006;130:1047-53.

67. Gilissen LP, Wong DR, Engels LG, Bierau J, Bakker JA, Paulussen AD, Romberg-Camps MJ, Stronkhorst A, Bus P, Bos LP, Hooymans PM, Stockbrügger RW, Neef C, Masclee AA. Therapeutic drug monitoring of thiopurine metabolites in adult thiopurine tolerant IBD patients on maintenance therapy. J Crohns Colitis 2012;6:698-707.

68. Moreau AC, Paul S, Del Tedesco E, Rinaudo-Gaujous M, Boukhadra N, Genin C, Peyrin-Biroulet L, Roblin $X$. Association between 6-thioguanine nucleotides levels and clinical remission in inflammatory disease: a meta-analysis. Inflamm Bowel Dis 2014;20:464-71.

69. Haines ML, Ajlouni Y, Irving PM, Sparrow MP, Rose R, Gearry RB, Gibson PR. Clinical usefulness of therapeutic drug monitoring of thiopurines in patients with inadequately controlled inflammatory bowel disease. Inflamm Bowel Dis 2011;17:1301-7.

70. van Asseldonk DP, Sanderson J, de Boer NK, Sparrow MP, Lémann M, Ansari A, Almer SH, Florin TH, Gearry RB, Mulder CJ, Mantzaris G, van Bodegraven AA; Thiopurine Task Force Interest Group. Difficulties and possibilities with thiopurine therapy in inflammatory bowel disease--proceedings of the first Thiopurine Task Force meeting. Dig Liver Dis 2011;43:270-6.

71. Chevaux JB, Peyrin-Biroulet L, Sparrow MP. Optimizing thiopurine therapy in inflammatory bowel disease. Inflamm Bowel Dis 2011;17:1428-35.

72. Vermeire S, Ferrante M, Rutgeerts P. Recent advances: Personalised use of current Crohn's disease therapeutic options. Gut 2013;62:1511-5.

73. Reinshagen M, Schütz E, Armstrong VW, Behrens C, von Tirpitz C, Stallmach A, Herfarth H, Stein J, Bias P, Adler G, Shipkova M, Kruis W, Oellerich M, von Ahsen N. 6-thioguanine nucleotide-adapted azathioprine therapy does not lead to higher remission rates than standard therapy in chronic active crohn disease: results from a randomized, controlled, open trial. Clin Chem 2007;53:1306-14.

74. González-Lama Y, Bermejo F, López-Sanromán A, García-Sánchez V, Esteve M, Cabriada JL, McNicholl AG, Pajares R, Casellas F, Merino O, Carpio D, Vera MI, Muñoz C, Calvo M, Benito LM, Bujanda L, GarcíaFernández FJ, Ricart E, Ginard D, Velasco M, Carneros JA, Manceñido N, Calvo M, Algaba A, Froilan C, Cara C, Maté J, Abreu L, Gisbert JP; "Grupo Español de Trabajo en Enfermedad de Crohn y Colitis Ulcerosa (GETECCU)". Thiopurine methyl-transferase activity and azathioprine metabolite concentrations do not predict clinical outcome in thiopurine-treated inflammatory bowel disease patients. Aliment Pharmacol Ther 2011;34:544-54.

75. Angelberger S, Schaeffeler E, Teml A, Petritsch W, Shonova O, Lukas M, Bar-Meir S, Dilger K, Greinwald R, Mueller R, Stange EF, Herrlinger KR, Schwab M, Reinisch W. Mucosal improvement in patients with moderate to severe postoperative endoscopic recurrence of Crohn's disease and azathioprine metabolite levels. Inflamm Bowel Dis 2013;19:590-8. 
76. van Asseldonk DP, de Boer NK, van Bodegraven AA. Thiopurine metabolite measurement - not for everyone. Aliment Pharmacol Ther 2011;34:1038-9; author reply 9-40.

77. LeLeiko NS, Lobato D, Hagin S, Hayes C, McQuaid EL, Seifer R, Kopel SJ, Boergers J, Nassau J, Suorsa K, Shapiro J, Bancroft B. 6-Thioguanine levels in pediatric IBD patients: adherence is more important than dose. Inflamm Bowel Dis 2013;19:2652-8.

78. Hanai H, lida T, Takeuchi K, Arai O, Watanabe F, Abe J, Maruyama Y, Oohata A, Ikeya K, Kageoka M, Miwa I, Yoshirou S, Hosoda Y, Kubota T. Thiopurine maintenance therapy for ulcerative colitis: the clinical significance of monitoring 6-thioguanine nucleotide. Inflamm Bowel Dis 2010;16:1376-81.

79. Smith M, Blaker P, Patel C, Marinaki A, Arenas M, Escuredo E, Anderson S, Irving P, Sanderson J. The impact of introducing thioguanine nucleotide monitoring into an inflammatory bowel disease clinic. Int J Clin Pract 2013;67:161-9.

80. Kennedy NA, Asser TL, Mountifield RE, Doogue MP, Andrews JM, Bampton PA. Thiopurine metabolite measurement leads to changes in management of inflammatory bowel disease. Intern Med J 2013;43:278-86.

81. Wong DR, Pierik M, Seinen ML, van Bodegraven AA, Gilissen LP, Bus P, Bakker JA, Masclee AA, Neef C, Engels LG, Hooymans PM. The pharmacokinetic effect of adalimumab on thiopurine metabolism in Crohn's disease patients. J Crohns Colitis 2014;8:120-8.

82. Kane SV, Cohen RD, Aikens JE, Hanauer SB. Prevalence of nonadherence with maintenance mesalamine in quiescent ulcerative colitis. Am J Gastroenterol 2001;96:2929-33.

83. Sewitch MJ, Abrahamowicz M, Barkun A, Bitton A, Wild GE, Cohen A, Dobkin PL. Patient nonadherence to medication in inflammatory bowel disease. Am J Gastroenterol 2003;98:1535-44.

84. Kane S, Huo D, Aikens J, Hanauer S. Medication nonadherence and the outcomes of patients with quiescent ulcerative colitis. Am J Med 2003;114:39-43.

85. Bokemeyer B, Teml A, Roggel C, Hartmann P, Fischer C, Schaeffeler E, Schwab M. Adherence to thiopurine treatment in out-patients with Crohn's disease. Aliment Pharmacol Ther 2007;15;26:217-25.

86. Kane S, Becker B, Harmsen WS, Kurian A, Morisky DE, Zinsmeister AR. Use of a screening tool to determine nonadherent behavior in inflammatory bowel disease. Am J Gastroenterol 2012;107: 154-60.

87. Sparrow MP. Use of allopurinol to optimize thiopurine immunomodulator efficacy in inflammatory bowel disease. Gastroenterol Hepatol (N Y) 2008;4:505-11.

88. Leung Y, Sparrow MP, Schwartz M, Hanauer SB. Long term efficacy and safety of allopurinol and azathioprine or 6-mercaptopurine in patients with inflammatory bowel disease. J Crohns Colitis 2009;3:162-7.

89. Hoentjen F, Seinen ML, Hanauer SB, de Boer NK, Rubin DT, Bouma G, Harrell LE, van Bodegraven AA. Safety and effectiveness of long-term allopurinol-thiopurine maintenance treatment in inflammatory bowel disease. Inflamm Bowel Dis 2013;19:363-9.

90. Seidman EG. Clinical use and practical application of TPMT enzyme and 6-mercaptopurine metabolite monitoring in IBD. Rev Gastroenterol Disord 2003;3 Suppl 1:S30-8.

91. Gilissen LP, Derijks L, Verhoeven HM, Bierau J, Hooymans PM, Hommes DW, Engels LG. Pancytopenia due to high 6-methylmercaptopurine levels in a 6-mercaptopurine treated patient with Crohn's disease. Dig Liver Dis 2007;39:182-6.

92. Seinen ML, van Bodegraven AA, van Kuilenburg AB, de Boer NK. High TPMT activity as a risk factor for severe myelosuppression during thiopurine therapy. Neth J Med 2013;71:222.

93. Wong DR, Coenen MJH, Vermeulen SH, Derijks LJ, van Marrewijk CJ, Klungel OH, Scheffer H, Franke B, Guchelaar HJ, de Jong DJ, Engels LGJB, Verbeek ALM, Hooymans PM, on behalf of the TOPIC consortium. Early assessment of thiopurine metabolites identifies patients at risk of leukopenia in inflammatory bowel disease. Submitted for publication 2014.

94. Colombel JF, Sandborn WJ, Reinisch W, Mantzaris GJ, Kornbluth A, Rachmilewitz D, Lichtiger S, D'Haens G, Diamond RH, Broussard DL, Tang KL, van der Woude CJ, Rutgeerts P; SONIC Study Group. Infliximab, azathioprine, or combination therapy for Crohn's disease. N Engl J Med 2010;362:1383-95.

95. Panaccione R, Ghosh S, Middleton S, Márquez JR, Scott BB, Flint L, van Hoogstraten HJ, Chen AC, Zheng $H$, Danese S, Rutgeerts $P$. Combination therapy with infliximab and azathioprine is superior to monotherapy with either agent in ulcerative colitis. Gastroenterology 2014;146:392-400 e3. 
96. D'Haens G, Baert F, van Assche G, Caenepeel P, Vergauwe P, Tuynman H, De Vos M, van Deventer S, Stitt L, Donner A, Vermeire S, Van de Mierop FJ, Coche JC, van der Woude J, Ochsenkühn T, van Bodegraven AA, Van Hootegem PP, Lambrecht GL, Mana F, Rutgeerts P, Feagan BG, Hommes D; Belgian Inflammatory Bowel Disease Research Group; North-Holland Gut Club. Early combined immunosuppression or conventional management in patients with newly diagnosed Crohn's disease: an open randomised trial. Lancet 2008;371:660-7.

97. Reenaers C, Louis E, Belaiche J, Seidel L, Keshav S, Travis S. Does co-treatment with immunosuppressors improve outcome in patients with Crohn's disease treated with adalimumab? Aliment Pharmacol Ther 2012;36:1040-8

98. Rutgeerts $P$, Van Assche G, Vermeire S. Optimizing anti-TNF treatment in inflammatory bowel disease. Gastroenterology 2004;126:1593-610.

99. Maser EA, Villela R, Silverberg MS, Greenberg GR. Association of trough serum infliximab to clinical outcome after scheduled maintenance treatment for Crohn's disease. Clin Gastroenterol Hepatol 2006;4:1248-54.

100. Fan J, Upadhye S, Worster A. Understanding receiver operating characteristic (ROC) curves. CJEM 2006;8:19-20.

101. Bastida G, Nos P, Aguas M, Beltrán B, Rubín A, Dasí F, Ponce J. Incidence, risk factors and clinical course of thiopurine-induced liver injury in patients with inflammatory bowel disease. Aliment Pharmacol Ther 2005 1;22:775-82.

102. Gisbert JP, Gonzalez-Lama Y, Mate J. Thiopurine-induced liver injury in patients with inflammatory bowel disease: a systematic review. Am J Gastroenterol 2007;102:1518-27.

103. Bermejo F, López-Sanromán A, Algaba A, Van-Domselaar M, Gisbert JP, García-Garzón S, Garrido E, Piqueras B, De La Poza G, Guerra I. Mercaptopurine rescue after azathioprine-induced liver injury in inflammatory bowel disease. Aliment Pharmacol Ther 2010;31:120-4.

104. Shaye OA, Yadegari M, Abreu MT, Poordad F, Simon K, Martin P, Papadakis KA, Ippoliti A, Vasiliauskas E, Tran TT. Hepatotoxicity of 6-mercaptopurine (6-MP) and Azathioprine (AZA) in adult IBD patients. Am J Gastroenterol 2007;102:2488-94.

105. Wong DR, Coenen MJH, Vermeulen SH, Derijks LJ, van Marrewijk CJ, Klungel OH, Scheffer H, Franke B, Guchelaar HJ, de Jong DJ, Engels LGJB, Verbeek ALM, Hooymans PM, on behalf of the TOPIC consortium. Early elevated 6-methylmercaptopurine levels predict thiopurine-induced hepatotoxicity in inflammatory bowel disease. Submitted for publication 2014.

106. de Jong DJ, Derijks LJ, Naber AH, Hooymans PM, Mulder CJ. Safety of thiopurines in the treatment of inflammatory bowel disease. Scand J Gastroenterol Suppl 2003(239):69-72.

107. van Egmond $\mathrm{R}$, Chin P, Zhang M, Sies CW, Barclay ML. High TPMT enzyme activity does not explain drug resistance due to preferential 6-methylmercaptopurine production in patients on thiopurine treatment. Aliment Pharmacol Ther 2012;35:1181-9.

108. Ansari A, Elliott T, Baburajan B, Mayhead P, O'Donohue J, Chocair P, Sanderson J, Duley J. Long-term outcome of using allopurinol co-therapy as a strategy for overcoming thiopurine hepatotoxicity in treating inflammatory bowel disease. Aliment Pharmacol Ther 2008;28:734-41.

109. Leong RW, Gearry RB, Sparrow MP. Thiopurine hepatotoxicity in inflammatory bowel disease: the role for adding allopurinol. Expert Opin Drug Saf 2008;7:607-16.

110. de Boer NK, Jharap B, Mulder C, van Bodegraven AA. Low and adequately dosed 6-thioguanine: not so bad after all. Inflamm Bowel Dis 2008;14:1166-7.

111. Gilissen LP, Derijks L, Driessen A, Bos LP, Hooymans PM, Stockbrügger RW, Engels LG. Toxicity of 6-thioguanine: no hepatotoxicity in a series of IBD patients treated with long-term, low dose 6-thioguanine. Some evidence for dose or metabolite level dependent effects? Dig Liver Dis 2007;39: 156-9.

112. Seinen ML, van Asseldonk DP, Mulder CJ, de Boer NK. Dosing 6-thioguanine in inflammatory bowel disease: expert-based guidelines for daily practice. J Gastrointestin Liver Dis 2010;19:291-4.

113. van Asseldonk DP, Seinen ML, de Boer NK, van Bodegraven AA, Mulder CJ. Hepatotoxicity associated with 6-methyl mercaptopurine formation during azathioprine and 6-mercaptopurine therapy does not occur on the short-term during 6-thioguanine therapy in IBD treatment. J Crohns Colitis 2012;6:95-101.

114. Lees CW, Maan AK, Hansoti B, Satsangi J, Arnott ID. Tolerability and safety of mercaptopurine in azathioprine-intolerant patients with inflammatory bowel disease. Aliment Pharmacol Ther 2008;27:220-7. 
115. Shih DQ, Nguyen M, Zheng L, Ibanez P, Mei L, Kwan LY, Bradford K, Ting C, Targan SR, Vasiliauskas EA. Split-dose administration of thiopurine drugs: a novel and effective strategy for managing preferential 6-MMP metabolism. Aliment Pharmacol Ther 2012;36:449-58.

116. de Boer NK, Wong DR, Jharap B, de Graaf P, Hooymans PM, Mulder CJ, Rijmen F, Engels LG, van Bodegraven AA. Dose-dependent influence of 5-aminosalicylates on thiopurine metabolism. Am J Gastroenterol 2007;102:2747-53.

117. de Graaf P, de Boer NK, Wong DR, Karner S, Jharap B, Hooymans PM, Veldkamp Al, Mulder CJ, van Bodegraven AA, Schwab M. Influence of 5-aminosalicylic acid on 6-thioguanosine phosphate metabolite levels: a prospective study in patients under steady thiopurine therapy. Br J Pharmacol 2010;160: 1083-91.

118. Kreijne JE, Seinen ML, Sinjewel A, Bouma G, Mulder CJ, van Bodegraven AA, de Boer NKH. Skewed thiopurine metabolism leads to therapeutic failure in the majority of thiopurine using IBD patients. P343. European Crohn's and Colitis Organisation Congress; Copenhagen. 2014

119. Lennard L, Lilleyman JS. Individualizing therapy with 6-mercaptopurine and 6-thioguanine related to the thiopurine methyltransferase genetic polymorphism. Ther Drug Monit 1996;18:328-34.

120. Schwab M, Klotz U. Pharmacokinetic considerations in the treatment of inflammatory bowel disease. Clin Pharmacokinet 2001;40:723-51.

121. Szumlanski CL, Weinshilboum RM. Sulphasalazine inhibition of thiopurine methyltransferase: possible mechanism for interaction with 6-mercaptopurine and azathioprine. Br J Clin Pharmacol 1995;39: 456-9.

122. Dewit O, Vanheuverzwyn R, Desager JP, Horsmans Y. Interaction between azathioprine and aminosalicylates: an in vivo study in patients with Crohn's disease. Aliment Pharmacol Ther 2002;16: 79-85.

123. Xin H, Fischer C, Schwab M, Klotz U. Effects of aminosalicylates on thiopurine S-methyltransferase activity: an ex vivo study in patients with inflammatory bowel disease. Aliment Pharmacol Ther 2005;21:1105-9.

124. Hande S, Wilson-Rich N, Bousvaros A, Zholudev A, Maurer R, Banks P, Makrauer F, Reddy S, Burakoff R, Friedman S. 5-aminosalicylate therapy is associated with higher 6-thioguanine levels in adults and children with inflammatory bowel disease in remission on 6-mercaptopurine or azathioprine. Inflamm Bowel Dis 2006;12:251-7.

125. Gilissen LP, Bierau J, Derijks LJ, Bos LP, Hooymans PM, van Gennip A, Stockbrügger RW, Engels LG. The pharmacokinetic effect of discontinuation of mesalazine on mercaptopurine metabolite levels in inflammatory bowel disease patients. Aliment Pharmacol Ther 2005;22:605-11.

126. Lewis LD, Benin A, Szumlanski CL, Otterness DM, Lennard L, Weinshilboum RM, Nierenberg DW. Olsalazine and 6-mercaptopurine-related bone marrow suppression: a possible drug-drug interaction. Clin Pharmacol Ther 1997;62:464-75.

127. Neurath MF, Kiesslich R, Teichgräber U, Fischer C, Hofmann U, Eichelbaum M, Galle PR, Schwab M. 6thioguanosine diphosphate and triphosphate levels in red blood cells and response to azathioprine therapy in Crohn's disease. Clin Gastroenterol Hepatol 2005;3:1007-14.

128. Tiede I, Fritz G, Strand S, Poppe D, Dvorsky R, Strand D, Lehr HA, Wirtz S, Becker C, Atreya R, Mudter J, Hildner K, Bartsch B, Holtmann M, Blumberg R, Walczak H, Iven H, Galle PR, Ahmadian MR, Neurath MF. CD28-dependent Rac1 activation is the molecular target of azathioprine in primary human CD4+ $T$ lymphocytes. J Clin Invest 2003;111:1133-45.

129. Dilger K, Schaeffeler E, Lukas M, Strauch U, Herfarth H, Müller R, Schwab M. Monitoring of thiopurine methyltransferase activity in postsurgical patients with Crohn's disease during 1 year of treatment with azathioprine or mesalazine. Ther Drug Monit 2007;29:1-5.

130. Daperno M, Sostegni R, Canaparo R, Serpe L, Lavagna A, Crocellà L, Castagno F, Vernetto A, Rigazio C, Ercole E, D'Antico S, Pera A, Zara G, Rocca R. Prospective study of the effects of concomitant medications on thiopurine metabolism in inflammatory bowel disease. Aliment Pharmacol Ther 2009;30:843-53.

131. Andrews JM, Travis SP, Gibson PR, Gasche C. Systematic review: does concurrent therapy with 5-ASA and immunomodulators in inflammatory bowel disease improve outcomes? Aliment Pharmacol Ther $2009 ; 29: 459-69$.

132. Actis GC, Marzano A, Pellicano R, Rizzetto M. How important is mesalamine in the maintenance of steroid-refractory colitis? Inflamm Bowel Dis 2008;14:1026. 
133. Stocco G, Martelossi S, Malusa' N, Marino S, Decorti G, Bartoli F, Ventura A. Interruption of mesalamine and reduction of the blood concentration of the active metabolites of azathioprine: possible causes of ulcerative colitis relapse. Dig Dis Sci 2008;53:3246-9.

134. Shah JA, Edwards CM, Probert CS. Should azathioprine and 5-aminosalicylates be coprescribed in inflammatory bowel disease?: an audit of adverse events and outcome. Eur J Gastroenterol Hepatol 2008;20:169-73.

135. Gao X, Zhang FB, Ding L, Liu H, Wang XD, Chen BL, Bi HC, Xiao YL, Zhao LZ, Chen MH, Huang M, Hu PJ. The potential influence of 5-aminosalicylic acid on the induction of myelotoxicity during thiopurine therapy in inflammatory bowel disease patients. Eur J Gastroenterol Hepatol 2012;24:958-64.

136. D'Haens GR, Panaccione R, Higgins PD, Vermeire S, Gassull M, Chowers $Y$, Hanauer SB, Herfarth $H$, Hommes DW, Kamm M, Löfberg R, Quary A, Sands B, Sood A, Watermeyer G, Lashner B, Lémann M, Plevy S, Reinisch W, Schreiber S, Siegel C, Targan S, Watanabe M, Feagan B, Sandborn WJ, Colombel JF, Travis S. The London Position Statement of the World Congress of Gastroenterology on Biological Therapy for IBD with the European Crohn's and Colitis Organization: when to start, when to stop, which drug to choose, and how to predict response? Am J Gastroenterol 2011;106:199-212; quiz 3.

137. van Bodegraven AA, van Everdingen JJ, Dijkstra G, de Jong DJ, Oldenburg B, Hommes DW. [Guideline 'Diagnosis and treatment of inflammatory bowel disease in adults'. I. Diagnosis and treatment]. Ned Tijdschr Geneeskd 2010;154:A1899.

138. Roblin X, Serre-Debeauvais F, Phelip JM, Bessard G, Bonaz B. Drug interaction between infliximab and azathioprine in patients with Crohn's disease. Aliment Pharmacol Ther 2003;18:917-25.

139. Teichgraber U, Atreya I, Atreya R, Schwab M, Neurath MF. Infliximab treatment induces levels of the active azathioprine metabolite TGTP in Crohn's disease. Inflamm Bowel Dis 2013;19:E54-5. 
Chapter 13

Conclusions and clinical considerations 
218 Chapter 13 


\section{Conclusions and clinical considerations}

In the thesis, we have studied the most important thiopurine metabolites of the conventional thiopurines azathioprine (AZA) and mercaptopurine (6MP) in patients with inflammatory bowel diseases (IBD) in relation to therapeutic response and common limiting adverse reactions.

The thiopurine metabolism in human is complex, as presented in Figure 1.1. The observed interindividual differences in therapeutic response and toxicity to thiopurines in IBD are to a large extent explained by the variable formation of active metabolites, which is at least partly caused by genetic polymorphisms of the genes encoding for crucial enzymes involving in thiopurine metabolism. Despite the rapidly increasing knowledge on thiopurine metabolism, polymorphisms of these genes and their consequences, only limited information exists on how to apply this knowledge in medical practice.

However, measurement of the active thiopurine metabolites reveals insight of the 'ultimate phenotype' of all crucial enzymes involving in the complex thiopurine metabolism.

AZA and 6MP undergo extensive intracellular metabolic transformations yielding to the most important pharmacologically active metabolites, the immunosuppressive 6-TGN and the methylated 6-MMPR. Thiopurine S-methyltransferase (TPMT) enzyme plays a pivotal role in the delicate balance of the 6-TGN and 6-MMPR formation. 6-TGN and 6-MMPR steady-state concentrations are generally reached after four to eight weeks and do not correlate well with thiopurine dose.

\section{Thiopurine metabolite testing in thiopurine maintenance therapy}

In clinical practice, 6-TGN and 6-MMPR metabolite measurement during maintenance thiopurine therapy is a helpful tool to explain therapeutic inefficacy (Chapter 5), toxicity or to reveal non-compliance (Chapter 5). The 6-TGN metabolites are responsible for the immunosuppressive effect. 6-TGN steady-state concentrations higher than $235 \mathrm{pmol} / 8 \times 10^{8} \mathrm{RBC}$ are associated with therapeutic response in adult IBD patients on thiopurine maintenance therapy. Thiopurine compliant patients on maintenance therapy with 6-TGN concentrations above the therapeutic threshold of $235 \mathrm{pmol} / 8 \times 10^{8}$ $\mathrm{RBC}$ are 4 times more likely to be in clinical remission (Chapter 5).

Non-compliance occurs frequently in the treatment of chronic diseases. Therapeutic drug monitoring (TDM) of thiopurine metabolites is the only way to reveal thiopurine nonadherence, especially when suspected, but denied by the patient (Chapter 5). However, very low or absent metabolite levels may not always be related to nonadherence or underdosing. Rare rapid xanthine oxidase metabolisers may be unable to generate detectable active thiopurine metabolites. Clinicians must be aware of this rare phenomenon (Chapter 4). 
IBD patients treated with AZA or 6MP who relapse or show poor response should be evaluated for adherence to therapy or underdosing, which both can be revealed by thiopurine metabolite testing. Nonadherent patients should be educated well. In poorly responding patients showing low thiopurine metabolite concentrations due to underdosing, thiopurine dosage should be optimised. Of course, changing their maintenance therapy to methotrexate or costly anti-TNF therapy should be considered in thiopurine resistant patients (Chapter 5 and 11).

Table 13.1 presents clinical recommendations on different thiopurine metabolite profiles in patients on azathioprine or mercaptopurine maintenance therapy showing inadequate response or when dose-dependent adverse effects are suspected.

Table 13.1 Thiopurine metabolite steady-state concentrations and management in IBD patients on azathioprine or mercaptopurine maintenance therapy showing non-response or possible dosedependent adverse effects.

\begin{tabular}{|c|c|c|c|c|}
\hline 6-TGN ${ }^{*}$ & 6-MMPR ${ }^{*}$ & Interpretation & $\begin{array}{l}\text { Dose-dependent } \\
\text { adverse effects }\end{array}$ & $\begin{array}{l}\text { Recommendation or } \\
\text { consideration }\end{array}$ \\
\hline$<<235$ & $<<5,700$ & $\begin{array}{l}\text { Nonadherence } \\
\text { xO hyperactivity? (rare) }\end{array}$ & & Patient education \\
\hline$<235$ & $<5,700$ & Underdosed & & $\begin{array}{l}\text { Increase thiopurine dose } \\
(\mathrm{AZA}+50 \mathrm{mg}, 6 \mathrm{MP}+25 \mathrm{mg})+ \\
\text { TDM after } 4 \text { weeks and } \\
\text { routine safety monitoring }\end{array}$ \\
\hline $235-500$ & $<5,700$ & $\begin{array}{l}\text { Thiopurine resistance, when } \\
6 \mathrm{TGN}>400\end{array}$ & & $\begin{array}{l}\text { Consider dose escalation + } \\
\text { TDM after } 4 \text { weeks and } \\
\text { routine safety monitoring } \\
\text { or change therapy }\end{array}$ \\
\hline$>500$ & $<5,700$ & $\begin{array}{l}\text { Overdosed } \\
\text { Intermediate TPMT? } \\
\text { Thiopurine resistance? }\end{array}$ & $\uparrow$ Risk of myelotoxicity & $\begin{array}{l}\text { Consider dose reduction + } \\
\text { TDM after } 4 \text { weeks } \\
\text { or change therapy }\end{array}$ \\
\hline$>500$ & $>5,700$ & $\begin{array}{l}\text { Overdosed } \\
\text { Thiopurine resistance? }\end{array}$ & $\begin{array}{l}\uparrow \text { Risk of myelotoxicity + } \\
\text { hepatotoxicity/intolerance }\end{array}$ & $\begin{array}{l}\text { Consider dose reduction + } \\
\text { TDM after } 4 \text { weeks } \\
\text { or change therapy }\end{array}$ \\
\hline $235-500$ & $>5,700$ & $\begin{array}{l}\text { Thiopurine resistance, when } \\
6 \mathrm{TGN}>400 \\
\text { 6-MMPR/6-TGN >20: } \\
\text { skewed 6MMP metabolism } \\
\text { ('shunter') }\end{array}$ & $\begin{array}{l}\uparrow \text { Risk of hepatotoxicity + } \\
\text { intolerance }\end{array}$ & $\begin{array}{l}\text { Reduced thiopurine dose }+ \\
\text { allopurinol co-treatment } \\
\text { or change therapy }\end{array}$ \\
\hline$<235$ & $>>5,700$ & $\begin{array}{l}\text { 6-MMPR/6-TGN >20: } \\
\text { skewed 6MMP metabolism } \\
\text { ('shunter') }\end{array}$ & $\begin{array}{l}\uparrow \uparrow \text { Risk of hepatotoxicity }+ \\
\text { intolerance + myelotoxicity } \\
\text { therapeutic failure }\end{array}$ & $\begin{array}{l}\text { Reduced thiopurine dose }+ \\
\text { allopurinol co-treatment } \\
\text { or change therapy }\end{array}$ \\
\hline
\end{tabular}

\footnotetext{
* Concentration of 6-TGN and 6-MMPR in pmol/8×10 RBC; \# Allopurinol combination therapy (100mg/day) requires a thiopurine dose reduction up to $25-30 \%$ of the original dose, guided by metabolite assessment and safety monitoring.
} 


\section{Pharmacokinetic interactions in thiopurine metabolism}

Mesalazine (aminosalicylates, 5-ASA) is a safe, well-tolerated and effective drug in patients with mild-to-moderate ulcerative colitis, but the benefit in Crohn's disease is limited.

5-ASA influences the metabolism of thiopurines in a dose-dependent manner, which can be demonstrated by thiopurine metabolite testing (Chapter 9 and 10).

IBD patients who are unresponsive or refractory to standard thiopurine therapy may benefit from the coadministration of 5-ASA leading to an increase of the pharmacologically active 6-TGN levels and stable (or a decrease of) the hepatotoxic 6-MMPR metabolites. Clinicians should be aware of a slightly increased risk of myelotoxicity when combining these drugs during thiopurine maintenance therapy.

Thiopurine and anti-TNF combination therapy is more effective than either in monotherapy, at least in naïve patients with moderate-to-severe IBD. In addition, concomitant use of an immunosuppressant has been recommended to minimise the immunogenicity of anti-TNF monoclonal antibodies to prevent infusion reactions and loss of response.

Previously a drug interaction between azathioprine and infliximab (IFX) has been reported. However, such an interaction was not demonstrated for adalimumab (ADA). Despite good therapeutic response, 6-TGN and 6-MMPR concentrations did not significantly change during the first 12 weeks of concurrent therapy (Chapter 11).

\section{Novel strategies to optimise thiopurine safety and efficacy in IBD}

Leukopenia is a potentially lethal dose-dependent adverse reaction of thiopurines in IBD patients, which occurs most frequently during the first weeks to months of treatment. 6-TGN steady-state concentrations $>500 \mathrm{pmol} / 8 \times 10^{8} \mathrm{RBC}$ are associated with an increased risk of leukopenia. In addition, severe myelosuppression has been associated with extremely elevated 6-MMPR concentrations.

Individuals carrying variant TPMT alleles show reduced TPMT enzyme activity and are at increased risk of leukopenia when treated with standard thiopurine dose due to the formation of high myelotoxic 6-TGN concentrations.

However, TPMT-deficient IBD patients can be safely treated with a very low maintenance dose of the alternative thiopurine drug 6-thioguanine (6-TG) by metabolite directed dose-optimisation and intensive haematological safety monitoring (Chapter 8).

Prior-to-treatment TPMT genotype-based dosing results in a significant risk reduction of leukopenia in carriers of the three most common TPMT variants in the Caucasian population, $T P M T^{*} 2, T_{P M T}^{*} 3 A$ and $T P M T^{*} 3 C$ (Chapter 3 ). However, only up to $25 \%$ of the cases who develop leukopenia can be explained by TPMT genotype. 
Leukopenia is not always related to high 6-TGN concentrations. In fact, 6-TGN and 6-MMPR are both independently associated with the occurrence of leukopenia during week 1-8 of thiopurine therapy (Chapter 6).

The following predictive threshold concentrations at week 1 (T1) were defined: $6-\mathrm{TGN}=213 \mathrm{pmol} / 8 \times 10^{8} \mathrm{RBC}$ and $6-\mathrm{MMPR}=3,525 \mathrm{pmol} / 8 \times 10^{8} \mathrm{RBC}$. Patients exceeding either one or both of these thresholds at week 1 are at increased risk of leukopenia during the first 8 weeks. Concurrent anti-TNF therapy (i.e. IFX, ADA) is an independent risk factor for the development of leukopenia in patients starting thiopurine therapy.

A predictive algorithm for thiopurine-induced leukopenia was developed, based on the defined 6-TGN and 6-MMPR threshold values and the independent risk factors thiopurine type (6MP $1.0-1.5 \mathrm{mg} / \mathrm{kg}$ ) and concomitant anti-TNF therapy. In addition to pre-treatment TPMT genotyping, early assessment of 6-TGN and 6-MMPR metabolites at week 1 by using the proposed predictive algorithm is a helpful tool to identify patients at increased risk of thiopurine-induced leukopenia (Chapter 6).

Hepatotoxicity is a common adverse reaction related to the 6-MMPR metabolites, most frequently occurring in the first months of thiopurine therapy. Steady-state 6-MMPR concentrations higher than $5,700 \mathrm{pmol} / 8 \times 10^{8} \mathrm{RBC}$ are associated with a 3-fold increased risk of hepatotoxicity, although sensitivity and specificity are poor.

Hepatotoxicity, gastrointestinal complaints (i.e. nausea, vomiting and/or anorexia) and general malaise are correlated with elevated 6-MMPR concentrations (Chapter 7). Patients exceeding the 6-MMPR threshold concentration of $3,615 \mathrm{pmol} / 8 \times 10^{8} \mathrm{RBC}$ at week 1 are at increased risk of hepatotoxicity ( $\sim$-fold), gastrointestinal complaints ( 2.5-fold) and general malaise ( 2-fold) during the first 5 months of therapy.

Age, BMI and gender are important determinants for the development of thiopurineinduced hepatotoxicity. A predictive algorithm was developed based on the T1 6-MMPR threshold of $3,615 \mathrm{pmol} / 8 \times 10^{8} \mathrm{RBC}$ and the relevant determinants age $>50$ years, gender (male), BMI $>25 \mathrm{~kg} / \mathrm{m}^{2}$ to assess the risk for thiopurine-induced hepatotoxicity (Chapter 7).

For patients who are at increased risk of leukopenia or hepatotoxicity, intensive safety monitoring and/or adequate thiopurine dose reduction is advocated - in specific situations ('6-MMP-shunters') a switch to alternative therapy may be appropriate (i.e. 6-TG or a combination of $25-30 \%$ of standard thiopurine dose with $100 \mathrm{mg}$ allopurinol) (Chapter 6 and 7).

Table 13.2 presents clinical considerations and recommendations based on thiopurine metabolite profiles assessed one week after initiation (T1) (i.e. $7+/-1$ day), which may be used in the future to optimise thiopurine tolerance and safety in IBD patients starting thiopurine treatment. 
Table 13.2 Proposed considerations to optimise thiopurine tolerance and safety based on thiopurine metabolite assessment one week after initiation (T1).

\begin{tabular}{|c|c|c|c|}
\hline T1 6-TGN ${ }^{*}$ & T1 6-MMPR ${ }^{*}$ & Interpretation & Consideration \\
\hline \multirow[t]{4}{*}{$>213$} & \multirow[t]{4}{*}{$>3,600$} & Overdosing? & \multirow{4}{*}{$\begin{array}{l}\text { Dose reduction, TDM after } 4 \text { weeks } \\
\text { Intensive haematological and LT } \\
\text { safety monitoring }\end{array}$} \\
\hline & & $\begin{array}{l}\uparrow \uparrow \text { Risk of leukopenia and hepatotoxicity } \\
\text { Risk factors leukopenia: } \\
\text { concurrent anti-TNF agents }\end{array}$ & \\
\hline & & $\begin{array}{l}\text { Risk factors hepatotoxicity: } \\
\text { age }>50 \text { years, male, } \mathrm{BMI}>25 \mathrm{~kg} / \mathrm{m}^{2}\end{array}$ & \\
\hline & & $\begin{array}{l}\text { Risk of GI complaints and general } \\
\text { malaise }\end{array}$ & \\
\hline \multirow[t]{2}{*}{$>213$} & \multirow[t]{2}{*}{$<3,600$} & Intermediate TPMT activity? & Dose reduction, TDM after 4 weeks \\
\hline & & $\begin{array}{l}\uparrow \text { Risk of leukopenia } \\
\text { Risk factors leukopenia: } \\
\text { concurrent anti-TNF agents }\end{array}$ & $\begin{array}{l}\text { Frequent haematological and } \\
\text { routine LT safety monitoring } \\
\text { TPMT genotyping for diagnosis }\end{array}$ \\
\hline \multirow[t]{2}{*}{$>>213$} & \multirow[t]{2}{*}{$<<3,600$} & Low / absent TPMT activity & Dose reduction, TDM after 4 weeks \\
\hline & & $\begin{array}{l}\uparrow \uparrow \text { Risk of leukopenia } \\
\text { Risk factors leukopenia: } \\
\text { concurrent anti-TNF agents }\end{array}$ & $\begin{array}{l}\text { Frequent haematological and } \\
\text { routine LT safety monitoring } \\
\text { TPMT genotyping for diagnosis }\end{array}$ \\
\hline$<213$ & $<3,600$ & Low risk of leukopenia and hepatotoxicity & $\begin{array}{l}\text { Routine safety monitoring } \\
\text { TDM after } 4-8 \text { weeks }\end{array}$ \\
\hline \multirow[t]{2}{*}{$<<213$} & \multirow[t]{2}{*}{$<<3,600$} & \multirow{2}{*}{$\begin{array}{l}\text { Underdosing } \\
\text { Rapid XO metaboliser (rare)? }\end{array}$} & Increase dose, TDM after 4 weeks \\
\hline & & & Routine safety monitoring \\
\hline \multirow[t]{6}{*}{$<213$} & \multirow[t]{6}{*}{$>3,600$} & $\begin{array}{l}\text { Risk of hepatotoxicity, GI complaints, } \\
\text { general malaise }\end{array}$ & $\begin{array}{l}\text { Frequent haematological and LT } \\
\text { safety monitoring }\end{array}$ \\
\hline & & $\begin{array}{l}\uparrow \quad \text { Risk of leukopenia } \\
\text { Risk factors leukopenia: } \\
\text { concurrent anti-TNF agents }\end{array}$ & \multirow{5}{*}{$\begin{array}{l}\text { 6-MMPR/6-TGN >20: } \\
\text { Switch to } 6 \text { TG or dose reduction in } \\
\text { combination with allopurinol + } \\
\text { TDM after } 4 \text { weeks } \\
\text { Frequent haematological and LT } \\
\text { safety monitoring }\end{array}$} \\
\hline & & Risk factors hepatotoxicity: & \\
\hline & & age $>50$ years, male, $\mathrm{BMI}>25 \mathrm{~kg} / \mathrm{m}^{2}$ & \\
\hline & & $\begin{array}{l}\text { Skewed 6MMP metabolism? } \\
\text { determine 6-MMPR/6-TGN ratio }\end{array}$ & \\
\hline & & $\uparrow \quad$ Risk non-response + intolerance & \\
\hline \multirow[t]{3}{*}{$<<213$} & \multirow[t]{3}{*}{$>>3,600$} & $\begin{array}{l}\text { Skewed 6MMP metabolism } \\
\uparrow \uparrow \text { Risk of leukopenia } \\
\text { Risk factors leukopenia: } \\
\text { concurrent anti-TNF agents }\end{array}$ & \multirow{3}{*}{$\begin{array}{l}\text { Switch to } 6 \text { TG or dose reduction in } \\
\text { combination with allopurinol + } \\
\text { TDM after } 4 \text { weeks } \\
\text { Frequent haematological and LT } \\
\text { safety monitoring }\end{array}$} \\
\hline & & $\begin{array}{l}\uparrow \uparrow \text { Risk of hepatotoxicity, GI complaints, } \\
\text { general malaise } \\
\text { Risk factors hepatotoxicity: } \\
\text { age }>50 \text { years, male, BMI }>25 \mathrm{~kg} / \mathrm{m}^{2}\end{array}$ & \\
\hline & & $\uparrow \uparrow$ Risk non-response + intolerance & \\
\hline
\end{tabular}

* Concentration of 6-TGN and 6-MMPR in pmol/8×10 ${ }^{8}$ RBC; LT = liver tests; 6TG, 6-thioguanine.. 


\section{Thiopurine metabolite assessment: practical considerations}

The availability of different analytical assays to measure thiopurine metabolite concentrations makes the data hard to interpret and to compare. In the studies presented in this thesis, the thiopurine metabolite concentrations were determined by the modified HPLC method of Lennard and Singleton (Lennard \& Singleton J Chrom 1992, Derijks et al. Ther Drug Monit 2004), which has most frequently been applied in clinical studies on thiopurine metabolites in IBD. Currently, the Dervieux-Boulieu method is commonly used in the Netherlands, which is less laborious and timeconsuming (Dervieux et al. Clin Chem 1998). Dervieux' method led to 1.4-2.6-fold higher 6-TGN concentrations in RBC, which is at least for a part depending on hydrolysis time and conditions (e.g. sulphuric acid versus perchloric acid), while 6-MMPR levels were found to be similar (Armstrong et al. Ther Drug Monit 2004). Therefore, the proposed therapeutic ranges and predictive thresholds of 6-TGN and 6-MMPR metabolites are not generally applicable when other analytical methodology is used. A validated correction factor is then required.

Many confounding factors are involved in thiopurine metabolite directed strategies, such as the large inter-individual variation of thiopurine metabolite formation (also intra-individual during therapy), the delayed onset of response after initiation (in general 2-4 months), the natural relapsing and remitting character of IBD, thiopurine resistance among IBD patients and the fact that thiopurine metabolite levels in erythrocytes actually are a surrogate marker for the leukocytes, the actual target cells.

Thiopurine metabolite testing is currently advocated in case of therapy resistance to reveal nonadherence or underdosing, and when dose-dependent adverse effects are suspected.

Clinical data show that correctly optimised thiopurines will safely maintain long-term remission in the vast majority of IBD patients. Therefore, a prolonged response/remission on thiopurines can occur, if correct dosing is achieved. Thiopurine metabolite assessment during steady-state (at least four weeks after initiation) can be helpful to optimise thiopurine dosage based on the therapeutic ranges in order to improve efficacy, but also to prevent adverse effects and late toxicity (Table 13.1).

Frequently performed therapeutic drug monitoring may reveal nonadherence, or under- or overdosing due to individual variation of thiopurine metabolism in time, allowing proactive strategies to prevent the occurrence of adverse events or flares of disease. 
The main findings of the studies presented in Chapter 6 and 7 of this thesis indicate that $T 1$ thiopurine metabolite testing offers a new strategy to optimise thiopurine therapy in IBD. A personalised pharmacological approach may prevent intolerable dosedependent adverse events in patients starting thiopurine treatment. However, large prospective (randomised-controlled) studies are needed to evaluate clinical outcome of the proposed strategies. 

Summary 



\section{Summary}

The thiopurine analogues azathioprine (AZA) and mercaptopurine (6MP) are frequently used immunosuppressive drugs to treat the chronic inflammatory bowel diseases (IBD) Crohn's disease (CD), ulcerative colitis (UC) and indeterminate colitis. Thiopurines are the mainstay of conventional IBD therapy and have proven efficacy for both induction and maintenance of remission. Furthermore, they act as steroid-sparing agents by reducing the need of corticosteroids. The onset of action is usually slow (8-16 weeks), which make them unsuitable for fast induction therapy in case of a flare of disease.

Unfortunately, thiopurines are not effective in up to one-third of the patients, and up to one-fourth discontinue therapy due to intolerable adverse reactions.

The development of adverse reactions and toxicity has been related to specific active thiopurine metabolites, of which the 6-thioguanine nucleotides (6-TGN) and 6-methylmercaptopurine ribonucleotides (6-MMPR) are considered to be the most important.

The 6-TGN metabolites are related to the immunosuppressive effects, whereas highly elevated concentrations are associated with the development of myelotoxicity. Elevations of 6-MMPR metabolites are mainly associated with hepatotoxicity and treatment failure.

However, new insights obtained by pharmacogenetics in thiopurine metabolism and assessment of 6-TGN and 6-MMPR metabolites in patients on thiopurine therapy have led to strategies to reduce toxicity and optimise efficacy and safety in IBD.

The objective of this thesis was to design and evaluate new ways to improve efficacy and safety of thiopurine therapy in patients with IBD.

Knowledge of the activity of the most important metabolic enzymes involved in thiopurine metabolism may be helpful to explain or predict therapeutic response and toxicity.

In Part I, the pharmacogenetics of thiopurine metabolism are evaluated.

Chapter 2 provided an 'in-depth' review on pharmacogenetics of thiopurine metabolism. Several polymorphisms of genes encoding for the enzymes involved in thiopurine metabolism are reviewed and the clinical implications in IBD are discussed. Although knowledge in this field is rapidly increasing, the translation into practical guidelines requires much effort and additional prospective clinical studies are needed to elucidate the relevance in clinical practice.

One of the most extensively investigated enzymes involving thiopurine metabolism is thiopurine S-methyltransferase (TPMT). TPMT enzyme activity is related to genetic variants of the TPMT gene. Individuals carrying variant alleles show reduced TPMT 
enzyme activity, and once treated with a standard thiopurine dose high myelotoxic 6-TGN levels will be formed at the expense of the 6-MMPR metabolite formation resulting in a highly increased risk of leukopenia. In Chapter $\mathbf{3}$ we evaluated in a multicentre randomised-controlled trial (TOPIC, $n=769$ ) whether prior to thiopurine treatment TPMT genotype-guided dosing reduced the risk of leukopenia in IBD patients starting thiopurine therapy. Compared to the control group, TPMT genotype-based dosing resulted in a significant reduction of leukopenia in patients carrying a TPMT variant $\left({ }^{*} 2,{ }^{*} 3 A\right.$ or $\left.{ }^{*} 3 C\right)$ in the intervention group $(22.9 \%$ versus $2.6 \%$, relative risk 0.11 (95\% Cl: 0.01-0.85), without impeding treatment efficacy. Unfortunately, we could not demonstrate a leukopenia risk reduction when evaluating all patients of the intervention and control group. The finding indicates that the TPMT genotype cannot explain all cases of leukopenia, which is in accordance with previous reports. Thiopurine-induced leukopenia must therefore also be attributed to other factors than a diminished TPMT enzyme activity, which should be elucidated in future prospective studies.

Chapter 4 described the case of a female patient with chronic autoimmune pancreatitis treated with high-dose azathioprine who was not able to form detectable thiopurine metabolites due to hyperactivity of the catabolic enzyme xanthine oxidase. This case clearly demonstrates the relevance of xanthine oxidase in the biotransformation of thiopurines.

In Part II, the clinical relevance of thiopurine metabolite assessment is discussed in relation to the therapeutic efficacy and safety.

In Chapter 5, the usefulness of therapeutic drug monitoring (TDM) was evaluated in patients on thiopurine maintenance therapy. A prospective cross-sectional study was performed in adult IBD patients on thiopurine maintenance therapy comparing the thiopurine metabolite concentrations in patients with a flare of disease $(n=41)$ with those of patients in remission ( $n=59$ ). The median 6-TGN levels were not significantly different between the exacerbation and remission group. The previously reported therapeutic threshold of 6-TGN was confirmed to be $\sim 235 \mathrm{pmol} / 8 \times 10^{8} \mathrm{RBC}$ in adult IBD patients, although sensitivity and specificity were rather poor. Compliant patients with steady-state 6-TGN concentrations above the therapeutic threshold were approximately 4 times more likely to have quiescent disease. Another important finding was that approximately one in six patients showed non-compliance of thiopurine therapy. These data support the role of TDM in thiopurine maintenance therapy in IBD to reveal non-compliance or underdosing, and can be used as a practical tool to optimise thiopurine therapy, especially in case of thiopurine non-response.

Leukopenia is a potentially lethal adverse reaction of thiopurine treatment, which has mainly been attributed to highly elevated 6-TGN steady-state concentrations. 
Thiopurine-induced leukopenia occurs in $2-5 \%$ of the patients, most frequently during the first weeks to months of treatment. Since steady-state thiopurine metabolite concentrations are generally reached after four to eight weeks of treatment, we evaluated whether early assessed thiopurine metabolites predict the development of leukopenia. Chapter 6 described the prospective study on the predictive value of 6-TGN and 6-MMPR metabolites, assessed one week after initiation (T1), for the development of leukopenia during the first 8 weeks of treatment in a subset of IBD patients of the TOPIC trial starting thiopurine therapy. We evaluated the $T 1$ metabolite concentrations of 6-TGN and 6-MMPR in patients with and without leukopenia ( $n=32$ and $n=162$, respectively). In order to predict the development of leukopenia, T1 threshold concentrations of $213 \mathrm{pmol} / 8 \times 10^{8}$ red blood cells (RBC) for 6-TGN and 3,525 $\mathrm{pmol} / 8 \times 10^{8} \mathrm{RBC}$ for 6-MMPR were defined: patients exceeding these values were at increased leukopenia risk (OR 6.2 (95\% Cl: 2.8-13.8) and 5.9 (95\% Cl: 2.7-13.3), respectively). In addition, leukopenia rates appeared to be higher in patients treated with 6MP, compared to AZA (OR 7.3 (95\% Cl: 3.1-17.0)), and in patients on concurrent anti-TNF therapy (OR 5.1 (95\% Cl: 1.6-16.4)). Based on logistic regression analysis of the threshold concentrations, thiopurine type and concurrent anti-TNF therapy, we developed a predictive algorithm ( $A \cup C=0.84$ ): elevations of both 6-TGN and 6-MMPR at $T 1$ resulted in the highest risk for leukopenia, followed by exceeding only the $T 1$ 6-MMPR or 6-TGN threshold concentration. Patients concurrently treated with anti-TNF therapy with elevations of both 6-TGN and 6-MMPR T1 concentrations were at highest risk to develop leukopenia.

In this prospective study we showed for the first time that elevated 6-TGN and 6-MMPR metabolite levels at week 1 are both highly predictive for thiopurine-induced leukopenia during the first 8 weeks of treatment in IBD patients. Based on different combinations of the four independent determinants, patients can be classified by using this predictive algorithm to assess the degree of risk for the development of leukopenia.

Up to one-fourth of IBD patients discontinue thiopurine medication within the first months after initiation due to intolerable adverse events. Hepatotoxicity, gastrointestinal complaints and general malaise are the most common thiopurine limiting adverse reactions, which have been related to high 6-MMPR metabolite concentrations. Chapter 7 described a prospective study performed in 270 IBD patients starting thiopurine therapy evaluating the predictive value of the 6-MMPR metabolite concentration, assessed one week after initiation (T1), for the development of hepatotoxicity, gastrointestinal complaints and general malaise during the first 20 weeks of treatment. Seventeen per cent of the patients developed hepatotoxicity. The 6-MMPR metabolites assessed at week one were associated with thiopurineinduced hepatotoxicity during the first 20 weeks of treatment. Patients with 6-MMPR concentrations exceeding the defined predictive $T 1$ threshold of $3,615 \mathrm{pmol} / 8 \times 10^{8} \mathrm{RBC}$ were approximately 4 times more at risk of hepatotoxicity during the first 20 weeks of 
treatment. 6-MMPR concentrations above the threshold correlated with gastrointestinal complaints (i.e. nausea, vomiting and anorexia; OR $2.4(95 \% \mathrm{Cl}$ : 1.4-4.3)) and general malaise (OR $2.0(95 \% \mathrm{Cl}: 1.1-3.7)$. The discriminative value of 6-MMPR for these adverse events was rather poor. However, thiopurine-induced hepatotoxicity was independently correlated with the male gender, higher age, body weight and body mass index (BMI). Age and BMI demonstrated the best discriminative values to predict hepatotoxicity in patients starting thiopurine therapy.

A predictive algorithm was developed based on the relevant determinants age, gender, $\mathrm{BMI}$ and the 6-MMPR threshold to assess the risk for thiopurine-induced hepatotoxicity (AUC $=0.83$ ). Males, irrespective of age, with a BMI $>25 \mathrm{~kg} / \mathrm{m}^{2}$ and $T 1$ 6-MMPR concentrations exceeding the defined threshold of $3,615 \mathrm{pmol} / 8 \times 10^{8} \mathrm{RBC}$, appeared to be at highest risk and all developed hepatotoxicity. Females meeting these criteria were then at highly increased risk of hepatotoxicity, followed by males or females older than 50 years with a $\mathrm{BMI}>25 \mathrm{~kg} / \mathrm{m}^{2}$ with $T 1$ 6-MMPR concentrations below the defined threshold.

In this prospective study we showed that assessment of the 6-MMPR concentration one week after initiation identifies patients at risk for thiopurine-induced hepatotoxicity, gastrointestinal complaints and general malaise and can be helpful to optimise thiopurine treatment in IBD. We developed a predictive algorithm for thiopurine-induced hepatotoxicity with good accuracy, based on 6-MMPR metabolite concentrations assessed at week one, and the relevant patient characteristics age, gender and BMI.

In Chapter 8, the case of a patient with Crohn's disease was described who developed severe pancytopenia on a $50 \mathrm{mg}$ 6MP dose due to a high myelotoxic 6-TGN metabolite concentration, resulting from complete TPMT enzyme deficiency. We demonstrated how this patient successfully was treated with a low dose of 6-thioguanine (6TG), an alternative thiopurine drug. The 6TG dose was adjusted and optimised by using frequent 6-TGN metabolite measurements eventually resulting to a 6TG maintenance dose of $20 \mathrm{mg}$ every two weeks.

In Part III, the clinical relevance of some pharmacokinetic interactions in thiopurine metabolism were described.

Previous studies indicated that 5-aminosalicylates (5-ASA) may influence the metabolism of thiopurines. In Chapter 9, we described the prospective pharmacokinetic study on the influence of 5-ASA (Pentasa ${ }^{\circledR}$ granules, sachets) on 6-TGN and 6-MMPR formation in 26 IBD patients during steady-state AZA or 6MP maintenance therapy. We observed a dose-dependent increase of the 6-TGN concentrations after starting concurrent 5-ASA therapy. Concomitant use of $2 \mathrm{~g}$ 5-ASA daily for four weeks resulted in a $40 \%$ increase of the median 6-TGN concentration, whereas addition of a daily dose of $4 \mathrm{~g}$ 5-ASA led to a $70 \%$ increase compared to 
baseline. Overall, the 6-MMPR concentrations were not affected, which suggests that the TPMT enzyme activity was not influenced by 5-ASA or its metabolites in vivo. Signs of myelotoxicity were observed in $8 \%$ of the patients, which points to an increased risk of myelosuppression resulting from combination therapy.

The level of the pharmacologically active 6-TGN significantly increases in a dosedependent manner during 5-ASA coadministration. IBD patients who are unresponsive or refractory to standard thiopurine therapy due to suboptimal 6-TGN levels may benefit from the coadministration of 5-ASA.

The 6-thioguanine nucleotides consist of three different phosphorylated metabolites, namely 6-thioguanine monophosphate (6-TGMP), 6-thioguanine diphosphate (6-TGDP) and 6-thioguanine triphosphate (6-TGTP). 6-TGTP, in particular, is considered to contribute to the immunosuppressive effects of the thiopurine drugs by inhibiting the function of the small GTPase Rac1 in activated lymphocytes leading to T-cell apoptosis. In chapter 9 a dose-dependent effect of 5-ASA on total 6-TGN metabolite formation was shown. In Chapter 10, the influence of 5-ASA (Pentasa ${ }^{\circledR}$ granules, sachets) on the individual 6-thioguanine phosphate metabolite concentrations was evaluated in a subcohort of these patients $(n=17)$. We observed that coadministration of 5-ASA $2 \mathrm{~g}$ daily for a period of 4 weeks in patients on steady-state AZA therapy induced an increase in median 6-TGDP, 6-TGTP and total 6-TGN levels. A subsequent doseescalation of the 5-ASA dosage to $4 \mathrm{~g}$ daily showed no further elevation of the individual 6-TGN levels in RBC, but unexpectedly a reduction in total 6-MMPR levels was observed. Elevation of total 6-TGN levels was caused by an increase of both 6-TGTP and 6-TGDP metabolite levels; 6-TGTP remained the main metabolite within the total pool of 6-TGN (approximately $85 \%$ of total 6-TGN).

IBD patients unresponsive or refractory to standard thiopurine therapy may benefit from the coadministration of 5-ASA, leading to an increase of the pharmacologically active 6-TGN (i.e. 6-TGTP), and thereby stable or even a decrease of cytotoxic 6-MMPR metabolite levels.

Infliximab is a monoclonal antibody against the pro-inflammatory cytokine tumor necrosis factor- $\alpha$ (TNF- $\alpha$ ), and has been shown good efficacy in induction and maintenance of remission in IBD. Previously a drug interaction between azathioprine and infliximab has been reported. Chapter $\mathbf{1 1}$ describes the pharmacokinetic influence of the anti-TNF agent adalimumab on thiopurine metabolism in twelve Crohn's disease patients on AZA or 6MP maintenance therapy. During the follow-up period of 12 weeks the 6-TGN and 6-MMPR metabolite concentrations did not significantly change compared to baseline. The activity of three important enzymes involving thiopurine metabolism, thiopurine S-methyltransferase, inosine-triphosphate pyrophosphatase and hypoxanthine-guanine phosphoribosyltransferase, did not change either after 4 weeks of adalimumab treatment. Two patients (17\%) showed clinically relevant myelotoxicity within 2-4 weeks on combination therapy. Remarkably, at baseline and 
throughout the whole study period in almost two-third of the patients subtherapeutic 6-TGN concentrations were found, suggesting suboptimal thiopurine dosing.

The prospective interaction study in Crohn's disease patients did not provide evidence for a pharmacokinetic interaction between adalimumab and the conventional thiopurines AZA and 6MP.

Chapter 12 provides a general discussion of the implications of the results presented in this thesis in relation to the current literature, daily clinical practice and in perspective to future research.

Based on the most important findings of this thesis and the current literature, clinical recommendations, considerations and future perspectives are presented in Chapter 13. 
Samenvatting 



\section{Samenvatting}

De thiopurines azathioprine (AZA) en mercaptopurine (6MP) worden veelvuldig toegepast als immunosuppressiva bij de behandeling van de inflammatoire darmziekten (IBD), de ziekte van Crohn, colitis ulcerosa en niet-classificeerbare colitis. De effectiviteit van thiopurines voor inductie en handhaven van remissie is in klinische studies aangetoond. Bovendien werken ze corticosteroïdsparend. Thiopurines vormen daarmee een belangrijke pijler bij de behandeling van IBD. Het effect treedt echter langzaam op (veelal na ongeveer 8-16 weken), waardoor ze ongeschikt zijn voor een snelle inductietherapie in het geval van opvlamming van ziekte.

Helaas zijn thiopurines in de klinische praktijk bij ongeveer een derde van de patiënten onvoldoende effectief en een kwart stopt de behandeling wegens onverdraaglijke bijwerkingen.

De bijwerkingen en toxische effecten zijn gerelateerd aan specifieke actieve thiopurine metabolieten, waarvan de 6-thioguanine nucleotiden (6-TGN) en 6-methylmercaptopurine ribonucleotiden (6-MMPR) als meest relevant worden beschouwd.

De immunosuppressieve effecten worden voornamelijk toegeschreven aan de 6-TGN, maar te hoge 6-TGN concentraties zijn geassocieerd met myelotoxiciteit. Verhoogde concentraties van de 6-MMPR metabolieten zijn voornamelijk geassocieerd met levertoxiciteit en het falen van de behandeling.

Nieuwe inzichten in de farmacogenetica van het thiopurine metabolisme en het meten van de 6-TGN en 6-MMPR metabolietconcentraties in het bloed hebben geleid tot de ontwikkeling van nieuwe strategieën ter optimalisatie van de thiopurine therapie bij IBD.

In het onderzoek dat in het kader van dit proefschrift is uitgevoerd, werden nieuwe strategieën onderzocht ter verbetering van de effectiviteit en veiligheid van de thiopurine behandeling bij patiënten met IBD.

Inzicht met betrekking tot de activiteit van de relevante enzymen die betrokken zijn bij het thiopurine metabolisme kan nuttig zijn om het therapeutische effect en/of toxiciteit van thiopurines te verklaren, dan wel te voorspellen.

In Deel I wordt de farmacogenetica van het thiopurine metabolisme geëvalueerd.

Hoofdstuk 2 is een 'in-depth' review over de farmacogenetica van het thiopurine metabolisme bij IBD. Verscheidene polymorfismen van genen die coderen voor enzymen die betrokken zijn in het thiopurine metabolisme worden besproken. Tevens komt de klinische betekenis ervan aan de orde.

Hoewel de kennis op dit gebied de laatste jaren enorm is toegenomen, is de vertaling naar praktische richtlijnen echter nog moeilijk te realiseren. $\mathrm{Er}$ is een grote behoefte 
aan prospectieve klinische studies om de relevantie in de klinische praktijk nader te bepalen.

Thiopurine S-methyltransferase (TPMT) is een van de meest onderzochte enzymen betrokken bij het thiopurine metabolisme. De TPMT enzymactiviteit is sterk gerelateerd aan de interindividuele genetische variatie van het TPMT gen. Individuen met één of twee variant allelen hebben een (sterk) verlaagde TPMT enzymactiviteit, resulterend in hogere 6-TGN concentraties en daarmee een verhoogd risico op het ontwikkelen van een leukopenie wanneer ze met een standaard thiopurine dosering worden behandeld. In de studie beschreven in Hoofdstuk 3 werd in een gerandomiseerd gecontroleerd multicenter onderzoek (TOPIC, $n=769$ ) onderzocht of een op het TPMT genotypegebaseerde dosering tot een reductie van het optreden van leukopenie leidt bij IBD patiënten die starten met een thiopurine behandeling.

Vergeleken met de controle groep resulteerde een op TPMT genotype-gebaseerde dosering in een significante reductie van leukopenie in patiënten met een TPMT variant allel $\left(* 2,{ }^{*} 3 A\right.$ or $\left.* 3 C\right)$ in de interventie groep $(22,9 \%$ versus $2,6 \%$, relatief risico 0,11 $(95 \% \mathrm{Cl}: 0,01-0,85)$. Er werd geen risicoreductie van leukopenie gezien wanneer alle patiënten van de interventie en controle groep werden geëvalueerd.

In deze grote prospectieve studie is aangetoond dat een op TPMT genotypegebaseerde dosering leidt tot een significante reductie van het optreden van leukopenie bij patienten met een TPMT variant allel. Het blijkt echter dat het TPMT genotype niet alle gevallen van leukopenie kan verklaren, wat ook uit eerder onderzoek is gebleken. Thiopurine-geïnduceerde leukopenie zal daarom naast een verlaagde TPMT enzymactiviteit ook moeten worden toegeschreven aan andere factoren, die in toekomstig onderzoek nader zullen moeten worden onderzocht.

Hoofdstuk 4 beschrijft de casus van een vrouw met chronische auto-immuun pancreatitis bij wie, ondanks een hoge azathioprine dosering, geen actieve thiopurine metabolieten werden gemeten als gevolg van een sterk verhoogde activiteit van het katabole enzym xanthine oxidase.

De casus benadrukt de relevantie van xanthine oxidase in de biotransformatie van thiopurines.

In Deel II worden de resultaten van het onderzoek naar de klinische relevantie van het meten van thiopurine metaboliet concentraties besproken.

In een prospectieve cross-sectionele studie beschreven in Hoofdstuk $\mathbf{5}$ werd het nut van therapeutic drug monitoring (TDM) onderzocht bij volwassen patiënten met een thiopurine onderhoudsbehandeling. De actieve thiopurine metabolieten van 41 patiënten met een opvlamming van IBD werden vergeleken met die van 59 patiënten in remissie. 
De mediane 6-TGN concentraties in beide patiëntengroepen verschilden niet statistisch significant van elkaar. De eerder gerapporteerde therapeutische 6-TGN drempelwaarde van $\sim 235 \mathrm{pmol} / 8 \times 10^{8} \mathrm{RBC}$ werd bevestigd. De sensitiviteit en specificiteit waren echter matig. Therapietrouwe patiënten met 6-TGN steady-state concentraties boven deze therapeutische drempelwaarde hadden ongeveer vier maal zoveel kans op een remissie ten opzichte van patiënten met een 6-TGN concentratie onder deze drempelwaarde. Een tweede belangrijke bevinding van deze studie was dat één op de zes patiënten niet therapietrouw bleek te zijn met betrekking tot de thiopurine medicatie.

TDM kan worden gebruikt bij patiënten met een thiopurine onderhoudsbehandeling om non-compliance of onderdosering aan te tonen, en kan worden toegepast om de behandeling te optimaliseren, voornamelijk bij patiënten die onvoldoende respons vertonen.

Leukopenie is een potentieel gevaarlijke bijwerking van thiopurines, die voornamelijk wordt toegeschreven aan verhoogde toxische 6-TGN steady-state concentraties. Thiopurine-geïnduceerde leukopenie treedt op bij 2-5\% van de patiënten met IBD, met name gedurende de eerste weken tot maanden van de behandeling. Aangezien steadystate concentraties van de actieve thiopurine metabolieten in het algemeen vier tot acht weken na aanvang van de thiopurine therapie worden bereikt, onderzochten we of vroeg in de therapie gemeten thiopurine metabolietconcentraties het optreden van leukopenie kunnen voorspellen. Hoofdstuk 6 beschrijft de prospectieve studie bij een deel van de IBD patiënten van de TOPIC trial, waarin de voorspellende waarde van 6-TGN en 6-MMPR metabolieten op het optreden van leukopenie werd onderzocht gedurende de eerste 8 weken van de behandeling. De thiopurine metabolieten werden een week na start van de behandeling $(T 1)$ gemeten en vergeleken bij patiënten met en zonder leukopenie ( $n=32$ resp. $n=162$ ).

Om een leukopenie te kunnen voorspellen werd de $T 1$ drempelwaarde van $213 \mathrm{pmol} / 8 \times 10^{8}$ rode bloedcellen (RBC) gedefinieerd voor $6-\mathrm{TGN}$ en $3525 \mathrm{pmol} / 8 \times 10^{8}$ RBC voor 6-MMPR: patiënten die deze drempelwaarden overschreden hadden een verhoogd risico op het ontwikkelen van een leukopenie (OR 6,2 (95\% Cl: 2,8-13,8) resp. 5,9 (95\% Cl: 2,7-13,3). Bij patiënten die werden behandeld met 6MP trad leukopenie vaker op, vergeleken met AZA (OR 7,3 (95\% Cl: 3,1-17,0)). Dit was ook het geval bij patiënten die gelijktijdig met anti-TNF therapie werden behandeld, vergeleken met thiopurine monotherapie (OR 5,1 (95\% Cl: 1,6-16,4)).

Met behulp van logistische regressie werd er een voorspellend algoritme ontwikkeld, waarbij de 6-TGN en 6-MMPR drempelwaarden, het thiopurine type en al dan niet een gelijktijdige behandeling met anti-TNF als determinanten werden meegenomen (AUC=0,84).

Bij patiënten behandeld met thiopurine monotherapie met T1 6-TGN én 6-MMPR concentraties hoger dan de drempelwaarden was het risico op een leukopenie het grootst, gevolgd door patiënten die alleen de T1 6-MMPR of 6-TGN drempelwaarde 
overschreden. Patiënten met verhoogde T1 6-TGN en 6-MMPR concentraties die gelijktijdig werden behandeld met anti-TNF, ontwikkelden allen een leukopenie.

In dit prospectieve onderzoek bij IBD patiënten werd voor de eerste keer aangetoond dat verhoogde 6-TGN en 6-MMPR metaboliet concentraties op week 1 beiden zeer voorspellend zijn voor thiopurine-geïnduceerde leukopenie. Op basis van de vier onafhankelijke determinanten, gebruikmakend van het voorspellend algoritme, kunnen patiënten een week na aanvang van de behandeling worden geclassificeerd om het risico op een leukopenie gedurende de eerste 8 weken van de behandeling te bepalen.

Gedurende de eerste maanden na start staakt tot ongeveer $25 \%$ van de IBD patiënten de thiopurine behandeling vanwege onverdraaglijke bijwerkingen. Hepatotoxiciteit, gastrointestinale klachten en algehele malaise zijn de meest voorkomende limiterende bijwerkingen, en zijn geassocieerd met hoge 6-MMPR metabolietconcentraties.

Hoofdstuk 7 beschrijft een prospectieve studie bij 270 patiënten startend met thiopurine therapie, waarbij de voorspellende waarde van de 6-MMPR metaboliet concentratie op week 1 (T1) werd bepaald voor het optreden van hepatotoxiciteit, gastrointestinale klachten en algehele malaise gedurende de eerste 20 weken van de thiopurine behandeling.

Hepatotoxiciteit trad op bij 17\% van de patiënten. Deze bleek te zijn geassocieerd met de T1 6-MMPR metabolietconcentratie. Patiënten die de gedefinieerde 6-MMPR drempelwaarde van $3615 \mathrm{pmol} / 8 \times 10^{8}$ RBC op week 1 overschreden, hadden ongeveer een 4 maal verhoogd risico op hepatotoxiciteit. 6-MMPR concentraties boven de drempelwaarde correleerden tevens met gastrointestinale klachten (misselijkheid, braken en anorexie; OR 2,4 $(95 \% \mathrm{Cl}: 1,4-4,3))$ en algehele malaise $(\mathrm{OR} 2,0(95 \% \mathrm{Cl}$ : 1,1-3,7)).

De discriminatieve waarden van de T1 6-MMPR concentratie voor deze bijwerkingen waren matig. Thiopurine-geïnduceerde hepatotoxiciteit bleek echter mede geassocieerd te zijn met de onafhankelijke determinanten mannelijk geslacht, leeftijd, lichaamsgewicht en body mass index (BMI). Leeftijd en BMI toonden de hoogste discriminatieve waarden voor het optreden van hepatotoxiciteit.

Op basis van de meest relevante determinanten leeftijd, geslacht, BMI en de T1 6-MMPR drempelwaarde werd er een algoritme ontwikkeld om het risico op hepatotoxiciteit te bepalen $\left(A \cup C=0,83\right.$ ). Mannen met een $B M I>25 \mathrm{~kg} / \mathrm{m}^{2}$, onafhankelijk van de leeftijd, die de T1 6-MMPR drempelwaarde van $3615 \mathrm{pmol} / 8 \times 10^{8} \mathrm{RBC}$ overschreden, hadden het grootste risico en ontwikkelden allen hepatotoxiciteit. Het risico was vervolgens het grootst onder vrouwen met dezelfde karakteristieken, gevolgd door mannen of vrouwen ouder dan 50 jaar met een $B M I>25 \mathrm{~kg} / \mathrm{m}^{2}$ en een $T 1$ 6-MMPR concentratie onder de gedefinieerde drempelwaarde.

In dit prospectieve onderzoek werd aangetoond dat met behulp van het meten van de 6-MMPR concentratie, een week na aanvang van de thiopurine behandeling, patiënten kunnen worden geïdentificeerd met een verhoogd risico op thiopurine-geïnduceerde hepatotoxiciteit, gastrointestinale intolerantie en algehele malaise. Voor het eerst werd 
er een voorspellend algoritme ontwikkeld voor het optreden van hepatotoxiciteit, gebaseerd op de T1 6-MMPR concentratie and de relevante patiëntkarakeristieken leeftijd, geslacht en BMI. In de klinische praktijk kan het algoritme van nut zijn om de thiopurine behandeling bij IBD in een vroeg stadium te optimaliseren.

In Hoofdstuk 8 wordt de casus beschreven van een patiënt met de ziekte van Crohn die een ernstige pancytopenie ontwikkelde tijdens een behandeling met $50 \mathrm{mg}$ 6MP/dag. Hoge myelotoxische 6-TGN metabolietconcentraties ten gevolge van een complete TPMT deficiëntie waren hiervoor de oorzaak

De patiënt werd succesvol behandeld met een zeer lage dosis 6-thioguanine (6-TG), een alternatief thiopurine analoog. De 6TG dosering werd aangepast op basis van frequente 6-TGN metingen, uiteindelijk resulterend in een onderhoudsdosering van $20 \mathrm{mg}$ elke 2 weken.

In Deel III wordt het onderzoek naar de klinische relevantie van enkele farmacokinetische interacties met thiopurines gepresenteerd.

Eerdere studies vonden aanwijzingen dat 5-aminosalicylaten (5-ASA) het metabolisme van AZA en $6 \mathrm{MP}$ beïnvloeden. In Hoofdstuk 9 werd in een prospectieve studie bij 26 patiënten met een thiopurine onderhoudsbehandeling de invloed van 5-ASA (Pentasa ${ }^{\circledR}$ granulaat, sachets) onderzocht op de vorming van 6-TGN en 6-MMPR.

We observeerden een dosisafhankelijke stijging van de 6-TGN concentratie ten opzichte van baseline: gelijktijdig gebruik van 2 gram 5-ASA/dag gedurende vier weken resulteerde in een $40 \%$ stijging van de 6 -TGN concentratie, terwijl 4 gram 5 -ASA/dag leidde tot een stijging van $70 \%$. De 6-MMPR concentratie veranderde hierbij niet significant, wat suggereert dat de activiteit van het enzym thiopurine S-methyltransferase, verantwoordelijk voor de vorming van 6-MMPR, in vivo niet wordt beïnvloed door 5-ASA of zijn metabolieten. Bij $8 \%$ van de patiënten werd een voorbijgaande milde myelotoxiciteit gezien, wat wijst op een licht verhoogd risico op myelosuppressie als gevolg van combinatie therapie.

De concentratie van het farmacologisch actieve 6-TGN neemt op een dosisafhankelijke wijze toe bij gelijktijdig gebruik van 5-ASA. IBD patiënten met onvoldoende respons of bij wie de thiopurine behandeling faalt als gevolg van een suboptimale 6-TGN concentratie hebben mogelijk baat bij een combinatie met 5-ASA.

De 6-thioguanine nucleotiden bestaan uit drie verschillende gefosforyleerde metabolieten, namelijk 6-thioguanine monofosfaat (6-TGMP), 6-thioguanine difosfaat (6-TGDP) en 6-thioguanine trifosfaat (6-TGTP). Met name 6-TGTP wordt verantwoordelijk gehouden voor het immunosuppressieve effect van de thiopurines door remming van het enzym GTPase Rac1 in geactiveerde lymfocyten, leidend tot stimulatie van de geprogrammeerde celdood van de T-cel. 
In hoofdstuk 9 werd een dosisafhankelijk effect van 5-ASA beschreven op de vorming van de totale concentratie 6-TGN metabolieten. Hoofdstuk 10 beschrijft de studie waarin bij een deel van deze patiënten $(n=17)$ de invloed werd onderzocht van 5-ASA (Pentasa ${ }^{\circledR}$ granulaat, sachets) op de individuele 6-thioguanine nucleotiden.

Gelijktijdig gebruik van 2 gram 5-ASA/dag gedurende 4 weken bij patiënten met een azathioprine onderhoudsbehandeling resulteerde in een stijging van de mediane 6-TGDP, 6-TGTP en de totale 6-TGN concentraties. De toename van de totale 6-TGN concentratie was het gevolg van de stijging van 6-TGTP én 6-TGDP, waarbij 6-TGTP nog steeds het grootste aandeel had binnen de totale pool van de 6-TGN metabolieten (ca. 85\%). Een direct daaropvolgende dosisverhoging naar 4 gram 5-ASA/dag liet geen verdere stijging zien van de individuele 6-TGN metabolieten, maar onverwachts wel een daling van de totale 6-MMPR metabolietconcentratie.

Patiënten met IBD die niet of onvoldoende reageren op de standaard thiopurine behandeling hebben mogelijk baat bij een combinatie met 5-ASA, leidend tot een toename van de farmacologisch actieve 6-TGN metabolieten, met name 6-TGTP, bij een gelijkblijvende of zelfs dalende concentratie van de cytotoxische 6-MMPR metabolieten.

Infliximab is een monoklonaal antilichaam gericht tegen het pro-inflammatoire cytokine tumor necrosis factor- $\alpha$ (TNF- $\alpha$ ), en is bewezen effectief als inductietherapie en als onderhoudsbehandeling van IBD.

In eerder onderzoek kwam een geneesmiddelinteractie aan het licht tussen azathioprine en infliximab. Hoofdstuk 11 beschrijft de invloed van het anti-TNF- $\alpha$ antilichaam adalimumab op het thiopurine metabolisme bij 12 patiënten met een azathioprine of mercaptopurine onderhoudsbehandeling voor de ziekte van Crohn.

$\mathrm{Na}$ het toevoegen van adalimumab veranderden de 6-TGN and 6-MMPR metaboliet concentraties gedurende een follow-up periode van de eerste 12 weken niet significant ten opzichte van baseline. De enzymactiviteit van drie relevante enzymen betrokken bij het thiopurine metabolisme, namelijk thiopurine S-methyltransferase, inosine-trifosfaat pyrofosfatase en hypoxanthine-guanine fosforibosyltransferase, waren 4 weken na aanvang van de adalimumab behandeling ook niet significant veranderd.

Twee patiënten (17\%) toonden binnen 2-4 weken klinisch relevante myelotoxiciteit na het starten van adalimumab bij de thiopurine behandeling.

Opvallend was dat bij circa tweederde van de patiënten subtherapeutische 6-TGN concentraties werden gemeten gedurende de gehele studieperiode, wat suggereert dat de thiopurine medicatie bij deze patiënten niet optimaal werd gedoseerd.

In deze prospectieve interactiestudie bij patiënten met de ziekte van Crohn kon geen farmacokinetische interactie worden aangetoond tussen adalimumab en de conventionele thiopurines azathioprine en mercaptopurine. 
In Hoofdstuk 12 volgt een discussie over de betekenis van de belangrijkste uitkomsten van dit proefschrift in relatie tot de thans beschikbare literatuur, de dagelijkse klinische praktijk, maar ook met het oog op toekomstig onderzoek.

Tenslotte worden in Hoofdstuk 13 op basis van de belangrijkste in dit proefschrift gepresenteerde bevindingen en de meest recente literatuur enkele klinische aanbevelingen en overwegingen gepresenteerd, voor nu en wellicht voor de toekomst. 

Valorisation 



\section{Valorisation addendum}

The inflammatory bowel diseases (IBD) Crohn's disease (CD), ulcerative colitis (UC), and indeterminate colitis are chronic, relapsing inflammatory disorders primarily affecting the gastrointestinal tract, which may lead to disability and severe complications.

IBD may begin at any age, although it usually starts in young adults with a peak incidence between 20-40 years. Over the past decades, both the incidence and prevalence of IBD are gradually increasing, particularly in developed countries worldwide and the highest incidence and prevalence rates are seen in North America and Europe. ${ }^{1}$ IBD is associated with a high economic burden to the society resulting from increased healthcare costs and decreased work productivity. ${ }^{2}$

There is currently no curative treatment for IBD available, therefore most patients need lifelong medicinal therapy and many will eventually need to undergo surgery, which have important consequences for their quality of life.

Health care professionals are expected to deliver optimal cure and care for their IBD patients. Society and government are reducing financial budgets so that health care providers are forced to optimise efficacy and work according to guidelines and treatment protocols. Therapy in IBD is focussed to reduce disease activity and improve quality of life, but should be cost effective.

Conventional IBD drugs, including aminosalicylates, corticosteroids, thiopurines and methotrexate, are used to achieve and maintain remission of disease in order to prevent a complicated disease course.

The immunomodulating thiopurines azathioprine (AZA) and mercaptopurine (6MP) have proven their efficacy in active disease to induce remission, but also to maintain clinical remission in both CD and UC. The alternative thiopurine drug, 6-thioguanine (6TG) is a therapeutic option in strictly defined indications as an off-label rescue drug in patients who fail due to intolerance or resistance to the conventional thiopurines AZA or 6MP.

Monoclonal antibodies directed against the pro-inflammatory cytokine TNF- $\alpha$ (i.e. infliximab, adalimumab) are used to treat patients with moderate-to-severe IBD and/or patients who are refractory to conventional therapy.

Combined anti-TNF and thiopurine therapy seems to have beneficial therapeutic value in the treatment of moderate-to-severe active $C D$ and UC, and has been recommended to minimise the immunogenicity of anti-TNF monoclonal antibodies to prevent loss of response and infusion reactions.

Since the introduction of the fast acting anti-TNF- $\alpha$ agents IBD treatment paradigm has evolved towards a rapid escalation therapy to achieve more stringent goals, including rapid induction of clinical remission, maintenance of steroid-free clinical remission, mucosal healing, healing of fistulae, reducing rates of hospitalisation and surgery, and improvement of quality of life. These clinical outcomes have partly been driven by large clinical trials of (new) biological agents, which are notably also achievable with the 
much less expensive conventional IBD drugs, such as aminosalicylates, and the immunomodulators methotrexate and thiopurines. ${ }^{3-6}$

Moreover, approximately one-third of the patients with IBD show primary nonresponse to an inductive anti-TNF regimen. After initial achievement of induction response up to $40 \%$ loses response during long-term maintenance therapy as a result of a shift in the mechanism of inflammation or increased drug clearance induced by immunogenicity or other factors, which may lead to inadequate concentrations. ${ }^{7}$ Consequently, more than $10 \%$ of patients will eventually lose response each year and require dose intensification and/or decrease in infusion interval, which of course has important financial consequences. ${ }^{8}$

Thus, it is important that medical treatment of IBD is tailored to the individual patient outweighing the risks and benefits of the available drugs.

\section{Optimising strategies of thiopurines in inflammatory bowel diseases}

The thiopurine drugs are considered the mainstay of medical management of IBD, especially in steroid-dependent and steroid-refractory patients. Thiopurines are effective in approximately two-third of the patients with IBD. The onset of the immunosuppressive effect is usually slow, as it only can be expected within 8-16 weeks. Following an initial response the efficacy is well sustained with remission rates of $95 \%$, $69 \%$ and $55 \%$ after 1,3 and 5 years, respectively. ${ }^{9}$

\section{Thiopurine metabolite testing}

Azathioprine and mercaptopurine are extensively metabolised ultimately to their main pharmacologically active metabolites, 6-methylmercaptopurine ribonucleotides (6-MMPR) and 6-thioguanine nucleotides (6-TGN). The 6-TGN are responsible for the immunosuppressive effects of thiopurines causing apoptosis and thereby preventing proliferation of activated T-lymphocytes, whereas 6-MMPR metabolites are associated with hepatotoxicity and treatment failure due to intolerable adverse reactions.

6-TGN and 6-MMPR metabolite concentrations can be measured in the erythrocytes.

\section{Optimisation of thiopurine safety and tolerability}

Myelosuppression is a potential lethal adverse reaction of thiopurines, of which leukopenia is the most common presentation, resulting in a potentially increased risk of infections. Leukopenia occurs mostly within the first months of thiopurine therapy, and has been mainly attributed to high toxic 6-TGN concentrations resulting from a diminished TPMT enzyme activity in patients carrying a genetic variant in the TPMT gene.

Pre-treatment TPMT genotype-guided thiopurine dosing significantly reduces the occurrence of leukopenia in patients carrying a genetic TPMT variant (Chapter 3). 
However, the TPMT genotype cannot explain all cases of leukopenia (only in up to $\sim 25 \%$ ). Therefore, thiopurine-induced leukopenia must also be attributed to other factors, such as concomitant medication and/or viral infections. Since thiopurineinduced myelosuppression can occur at any time, and TPMT genotype or phenotype testing alone does not identify all patients at risk developing leukopenia, means that monitoring of full blood counts at week 1, 2, 4 and 8, and subsequently throughout the course of thiopurine treatment remains mandatory.

We showed that both 6-TGN and 6-MMPR assessed at week 1 are independently correlated with the development of leukopenia during week 1-8. The cytotoxic effect of these metabolites is presumably enhanced by one another. Furthermore, patients who developed leukopenia were more often treated with mercaptopurine than azathioprine. In addition, treatment with concurrent anti-TNF antagonists (i.e. infliximab or adalimumab) was demonstrated to be an independent risk factor for the development of leukopenia in patients starting thiopurine therapy.

These findings may explain, at least for a part, the fact that thiopurine-induced leukopenia is not always related to low TPMT enzyme activity (Chapter 6).

Early identification of patients at increased risk of leukopenia is important as it will ultimately reduce morbidity and outpatient monitoring, and to a (much) lesser extent hospitalisation or mortality. Therefore, we advise that in addition to pre-treatment TPMT genotyping, early assessment of 6-TGN and 6-MMPR metabolites at week 1 should be performed to identify patients at increased risk of thiopurine-induced leukopenia. The proposed accurate predictive algorithm may be a helpful tool for clinicians to use in daily practice (Chapter 6).

Unfortunately, up to $25 \%$ of the IBD patients discontinue therapy already during the first months of treatment mostly due to intolerable adverse reactions. Common adverse drug reactions prompting to discontinuation are gastrointestinal intolerance (i.e. nausea and vomiting) and hepatotoxicity. ${ }^{10}$

Assessment of the 6-MMPR concentration one week after thiopurine initiation identifies patients at risk of hepatotoxicity, gastrointestinal complaints and general malaise as a result from elevated 6-MMPR metabolite formation (Chapter 7). The proposed algorithm can be helpful to optimise thiopurine therapy in an early stage of treatment to prevent unnecessary thiopurine withdrawal due to the occurrence of these potentially intolerable adverse events. Switching between the thiopurines azathioprine, mercaptopurine and 6-tioguanine, as well as the addition of low-dose allopurinol in combination with a thiopurine dose reduction (25-33\% of standard) can prevent these adverse reactions and elevate 6TGN levels into the therapeutic range and lower the cytotoxic 6-MMPR (Chapter 13).

The proposed predictive algorithms for leukopenia and thiopurine-induced hepatotoxicity, and the proposed optimisation strategies should be validated in future 
prospective randomised-controlled trials to evaluate whether the proposed strategies actually result in an improved efficacy and safety profile (Chapter 12 \& 13).

\section{Thiopurine metabolite testing during maintenance therapy}

As a result of the large interindividual variation of active thiopurine metabolite formation due to the complex metabolism and genetic polymorphisms in the metabolising enzymes, conventional bodyweight-based thiopurine dosing frequently leads to an inadequate response or adverse events in many IBD patients.

Steady-state thiopurine metabolite concentrations are generally reached after approximately 4-8 weeks after treatment initiation. The therapeutic range of steadystate 6-TGN concentrations has been defined between 235 and $490 \mathrm{pmol} / 8 \times 10^{8}$ red blood cells (RBC) and 6-MMPR $<5,700 \mathrm{pmol} / 8 \times 10^{8} \mathrm{RBC}$.

Until now, thiopurine metabolite assessment is the only way to reveal insight into the ultimate phenotype of an individual's thiopurine metabolism and should be used to personalise a patient's thiopurine dose regimen.

In the study in adult IBD patients on thiopurine maintenance therapy, presented in Chapter 5, the previous reported therapeutic 6 -TGN cut-off level of $235 \mathrm{pmol} / 8 \times 10^{8}$ $\mathrm{RBC}$ in steady-state was confirmed, but showed rather poor sensitivity, specificity and positive predictive value. This is in line with the results of previous reports and may be explained by several confounding factors as described in Chapter 12. Nevertheless, higher 6-TGN levels were found to be significantly associated with clinical remission.

Therapeutic drug monitoring (TDM) is therefore a useful tool in thiopurine maintenance therapy in IBD to reveal underdosing and can be used to tailor thiopurine dose based on the active thiopurine metabolite levels.

\section{Thiopurines and nonadherence}

Since medication nonadherence is associated with an increased rate of relapse, clinicians should be well aware that nonadherence of aminosalicylates and thiopurines is underestimated as it occurs in up to $50 \%$ of the IBD patients.

In the study presented in Chapter 5 was demonstrated that approximately one in six patients was not adherent to thiopurine therapy, which was observed in patients with both active and quiescent disease.

Adherence behaviour of thiopurine therapy is hard to predict in clinical practice. Therefore, it is a challenging task for health care providers to promote adherence of pharmacotherapy among their patients. Thiopurine metabolite testing is the only useful tool to reveal nonadherence, especially when it is suspected, but denied by the patient. Once it is proven, patients should be supported and educated about the possible unfavourable consequences for their clinical course. Patient-related factors, such as acceptance of their chronic disease accompanied with the lifelong medicinal treatment, 
should be taken into consideration. Frequently performed TDM may reveal insight whether the incentive approach works and frequent education may even help to prevent an exacerbation of disease.

\section{Therapeutic failure of thiopurine maintenance therapy}

In daily practice, when patients on thiopurine maintenance therapy present with a flare of disease, an unnecessary switch is easily made to expensive anti-TNF therapy (Chapter 11).

Clinical data show that well optimised thiopurines will maintain long-term remission in the majority of IBD patients.

In order to improve the efficacy of thiopurine therapy, thiopurine metabolite testing should be undertaken. First, thiopurine nonadherence should be excluded in these patients. Second, in poorly responding patients with low 6-TGN and low 6-MMPR concentrations due to underdosing, thiopurine dose should be optimised before considering a change to methotrexate or expensive anti-TNF therapy.

\section{Economic burden of IBD medication to society}

The increasing knowledge of the pathophysiology of IBD will certainly lead to new immunological targets and consequently new expensive biological drugs against these targets in the future.

Recently it has been demonstrated that the traditional cost profile of IBD on the Dutch society has shifted over the past decades from surgical and medical hospitalisation related costs to health care costs mainly driven by medication use, in particular antiTNF therapy. ${ }^{2}$

Although these (new) biologicals are a great advance in the treatment of IBD, their costs are high compared to the conventional IBD therapies and contribute to the high economic burden of IBD to the society. ${ }^{2}$ For comparison, the average annual costs of anti-TNF antibodies infliximab and adalimumab are 15,000-19,000 euro per patient versus approximately 200-1000 euro per patient for thiopurine therapy. ${ }^{11}$

Considering the limited primary response rates and the loss of response of long-term anti-TNF therapy, the restricted number of alternative therapeutic options in IBD and the high costs of the novel immunosuppressive biological drugs, it is important that clinicians do not disregard conventional IBD therapy and dismiss it as having failed without a proper attempt of optimisation.

In this respect patients should be encouraged to participate actively in therapeutic decision making, which should be tailored to the individual. ${ }^{12}$

Finally, optimisation of safety and efficacy of first-line medical therapy with thiopurines as proposed in this thesis may contribute to efficient and cost-effective health care in IBD. 


\section{References}

1. Molodecky NA, Soon IS, Rabi DM, Ghali WA, Ferris M, Chernoff G, et al. Increasing incidence and prevalence of the inflammatory bowel diseases with time, based on systematic review. Gastroenterology 2012;142:46-54 e42; quiz e30.

2. van der Valk ME, Mangen MJ, Leenders M, Dijkstra G, van Bodegraven AA, Fidder HH, et al. Healthcare costs of inflammatory bowel disease have shifted from hospitalisation and surgery towards antiTNFalpha therapy: results from the COIN study. Gut 2014;63:72-9.

3. Neurath MF, Travis SP. Mucosal healing in inflammatory bowel diseases: a systematic review. Gut 2012;61:1619-35.

4. Prefontaine E, Macdonald JK, Sutherland LR. Azathioprine or 6-mercaptopurine for induction of remission in Crohn's disease. Cochrane Database Syst Rev 2009:CD000545.

5. Prefontaine E, Sutherland LR, Macdonald JK, Cepoiu M. Azathioprine or 6-mercaptopurine for maintenance of remission in Crohn's disease. Cochrane Database Syst Rev 2009:CD000067.

6. Timmer A, McDonald JW, Macdonald JK. Azathioprine and 6-mercaptopurine for maintenance of remission in ulcerative colitis. Cochrane Database Syst Rev 2007:CD000478.

7. Yanai H, Hanauer SB. Assessing response and loss of response to biological therapies in IBD. Am J Gastroenterol 2011;106:685-98.

8. Gisbert JP, Panes J. Loss of response and requirement of infliximab dose intensification in Crohn's disease: a review. Am J Gastroenterol 2009;104:760-7.

9. Fraser AG, Orchard TR, Jewell DP. The efficacy of azathioprine for the treatment of inflammatory bowel disease: a 30 year review. Gut 2002;50:485-9.

10. Jharap B, Seinen ML, de Boer NK, van Ginkel JR, Linskens RK, Kneppelhout JC, et al. Thiopurine therapy in inflammatory bowel disease patients: analyses of two 8-year intercept cohorts. Inflamm Bowel Dis 2010;16:1541-9.

11. www.medicijnkosten.nl; database on the Internet: cited September 15th 2014.

12. Dignass A, Lindsay JO, Sturm A, Windsor A, Colombel JF, Allez M, et al. Second European evidencebased consensus on the diagnosis and management of ulcerative colitis part 2: current management. J Crohns Colitis 2012;6:991-1030. 
Dankwoord 



\section{Dankwoord}

De basis van dit proefschrift werd gelegd met de onderzoeken in het kader van mijn opleiding tot ziekenhuisapotheker, en later tot klinisch farmacoloog, in het Maaslandziekenhuis te Sittard.

Luc Derijks en Lennard Gilissen hadden het Limburgse Zitterd onder begeleiding van dr. Piet Hooymans en dr. Leopold Engels al mooi op de kaart gezet met hun thiopurineonderzoek. Het was aan mij de eer om het 'stokje' over te nemen en me te verdiepen in de klinische farmacologie van de thiopurines bij de behandeling van inflammatoire darmziekten. In 2009 besloot ik daadwerkelijk om er een promotietraject van te maken. Nu ruim vijf jaar later is het eindelijk zo ver: het proefschrift is af!

Ik heb tijdens het traject dat vooraf is gegaan aan het voltooien van dit proefschrift enorm veel geleerd en ben dan ook erg dankbaar voor de opgedane kennis, zowel op wetenschappelijk als op persoonlijk vlak. Hoewel ik er altijd wel plezier aan heb beleefd, was het niet altijd even leuk en gemakkelijk om promotieonderzoek te doen naast een fulltime baan. Hier was het gezegde 'de laatste loodjes wegen het zwaarst' duidelijk van toepassing. Des te groter is de vreugde en opluchting die ik voel nu de afronding ervan is genaderd.

Veel mensen hebben mij de afgelopen jaren hierbij geholpen. Graag wil ik iedereen bedanken die heeft bijgedragen aan dit proefschrift. Zonder iemand tekort te willen doen, wil ik een aantal personen in het bijzonder bedanken.

Allereerst wil ik alle patiënten die hebben deelgenomen aan de verschillende onderzoeken bedanken voor hun deelname en voor de extra tijd die het ze heeft gekost. Júllie deelname heeft de totstandkoming van dit proefschrift mogelijk gemaakt. Graag wil ik eerst mijn co-promotoren dr. Piet Hooymans en dr. Leopold Engels bedanken, trots als ik ben om hun derde thiopurine-promovendus te mogen zijn.

Beste Piet, mijn opleider, mentor, en later mijn collega, wat heb ik geboft met jou als co-promotor! Regelmatig heb je mij het advies gegeven: 'Dennis, probeer je te focussen op de hoofdzaken', als ik weer afdwaalde. Ik schreef, en jij streepte. Op ieder moment kon ik bij je terecht en was je bereid om mij te adviseren als ik weer eens vastliep met schrijven of voor een (diplomatiek) dilemma stond. Ik waardeer je enorm als persoon en ben je erg dankbaar voor je vriendschap, je onvoorwaardelijke steun en alles wat je de afgelopen jaren voor mij hebt gedaan. Ik kijk ernaar uit om samen door te gaan met het onderzoek, ook nu je met pensioen bent.

Beste Leopold, jij was ooit samen met Piet de initiator van het thiopurine-onderzoek in Sittard. Ik zal nooit vergeten dat je me keer op keer belde en met een zware, luide stem zei: 'Dennis the Ménace!! Wat ben je aan het doen, jongen?? Ik heb hier een patiënt voor mij zitten, die graag mee wilt doen met je onderzoek'. Waarop ik naar je poli spurde met de patiëntenformulieren en bloedbuizen in mijn hand, hopende dat de patiënt instemde en voldeed aan de gestelde inclusiecriteria. Jij was voor mij de schakel tussen de farmacologie en de kliniek. Met jouw hulp werden er vele Sittardse patiënten geïncludeerd. Ik heb veel van je mogen leren op het gebied van diverse klinische 
(gastro-enterologische) aspecten, waar je als ziekenhuisapotheker niet altijd bekend mee bent. Ik wil je enorm bedanken voor jouw belangrijke bijdragen, je enthousiasme en de humor waarmee je mij hebt begeleid als co-promotor.

Mijn dank gaat ook uit naar mijn promotoren Prof. dr. Ad Masclee en Prof. dr. Kees Neef. Beste Ad, beste Kees, toen we jullie benaderden met de vraag of jullie mij wilden begeleiden als promotor was het fundament van het onderzoek reeds gelegd. Gelukkig was het antwoord positief en stemden jullie in met de geformuleerde onderzoeksvragen ten behoeve van de nog uit te voeren onderzoeken. Voor mij een unieke kans om te leren van jullie brede kennis en ervaring op het gebied van de gastroenterologie en de klinische farmacologie. Graag wil ik jullie beiden enorm bedanken voor de plezierige samenwerking en begeleiding tijdens mijn promotietraject. Ondanks de afstand stonden jullie altijd klaar om met mij mee te denken. De feedback en adviezen die jullie mij hebben gegeven tijdens het schrijven van de manuscripten en de thesis waren voor mij erg waardevol. Ad, op het juiste moment wist je mij iedere keer het ontbrekende puzzelstukje aan te reiken, dank je wel. Kees, achteraf blijkt dat we initieel meer interessante onderzoeksvragen hadden dan nodig was voor dit proefschrift, daarom hoop ik van harte dat we in de toekomst onze samenwerking kunnen voortzetten.

De leden van de beoordelingscommissie, Prof. dr. A. Bast, Prof. dr. J.R.B.J. Brouwers, Prof. dr. G. Dijkstra, dr. M. Pierik en Prof. dr. F.J. van Schooten wil ik hartelijk danken voor de inhoudelijke beoordeling van mijn manuscript.

De onderzoeken zijn uitgevoerd in nauwe samenwerking met diverse specialisten en/of onderzoekers van het Laurentius Ziekenhuis te Roermond, Academisch Ziekenhuis Maastricht, Catharina Ziekenhuis Eindhoven, VU Medisch Centrum Amsterdam en het Radboud Universitair Medisch Centrum Nijmegen. In totaal hebben 30 Nederlandse centra deelgenomen aan het TOPIC-onderzoek. Graag wil ik alle MDL-artsen, IBDverpleegkundigen en medewerkers van alle betrokken laboratoria Klinische chemie en Klinische farmacologie \& Toxicologie bedanken voor de onmisbare ondersteuning bij de inclusie van patiënten, de bloedafname, en de registratie en verwerking van de bloedspiegels. Daarnaast wil ik de medewerkers van het Laboratorium voor Erfelijke Metabole Ziekten van het Academisch Ziekenhuis Maastricht bedanken voor hun belangrijke ondersteuning.

Ik wil graag Rens Bos, Mariëlle Romberg en Paul Bus bedanken voor hun enthousiasme bij het includeren van hun patiënten namens Sittard en Roermond. Tevens voor de hulp bij het verzamelen van klinische data uit de patiëntendossiers en de bijdrage aan dit proefschrift.

Joop ten Kate, Renée Vossen, Bernadette van den Acker, Marlies Huizinga en de andere medewerkers van het Klinisch Chemisch Hematologisch Laboratorium van het Orbis Medisch Centrum, bedankt voor jullie rol bij de implementatie van de verschillende onderzoeken. 
Beste Lennard, samen met Luc ben jij de grondlegger geweest van onze onderzoekslijn. $\mathrm{Na}$ jouw vertrek uit Sittard, zorgde jij voor de samenwerking met het Catharina Ziekenhuis in Eindhoven en de brug naar Maastricht, dankjewel, ook voor je bijdrage aan mijn proefschrift. Je bent nu ontzettend druk bezet als MDL-arts in het Catharina Ziekenhuis, maar hopelijk vinden we beiden in de toekomst nog eens tijd om gezamenlijk een onderzoek op te zetten.

Beste Jörgen Bierau, beste Jaap Bakker, thiopurinofielen in hart en nieren, bedankt voor de inzichten die jullie me hebben gegeven met betrekking tot de TPMT, HGPRT en ITPA genotypering, de enzymactiviteit metingen in onze studies en de waardevolle bijdragen aan dit proefschrift.

Beste Marieke Pierik, graag wil ik je bedanken voor de inclusie van patiënten, de hulp bij de data-analyse, het geven van waardevolle feedback op de geschreven stukken en daarmee jouw bijdrage aan dit proefschrift. Hopelijk zal onze samenwerking in de toekomst voortduren, zodat we onze ideeën met betrekking tot verder onderzoek in praktijk kunnen brengen.

Beste Wout Mares, ik wil jou ook graag bedanken voor de inclusie van patiënten en voor jouw bijdrage als mede-onderzoeker. Veel succes gewenst als MDL-arts in Ede, en wie weet zullen we samen ooit nog eens een mooi case report schrijven.

Beste Nanne de Boer, mijn eerste thiopurine-onderzoek heb ik samen met jou gedaan. We onderzochten de invloed van mesalazine op het thiopurine metabolisme. Samen met Peer de Graaf werd de farmacologie vertegenwoordigd. Veel ontzag heb ik altijd gehad voor de snelheid waarmee de publicaties uit het Amsterdamse VUMC PubMed binnenvlogen, allemaal onder begeleiding van Prof. dr. Chris Mulder en dr. Ad van Bodegraven. Hetzelfde geldt voor jouw opvolgers Bindia Jharap, Dirk van Asseldonk en Margien Seinen, die alle drie hoogstwaarschijnlijk ook komend jaar zullen promoveren. Het mooie is dat onze onderzoeken elkaar nooit hebben gebeten. Sterker nog, waar nodig sprongen jullie zelfs bij met het includeren van patiënten. Nanne, Bindia, Dirk, Peer, Margien, Ad en Chris, ik wil jullie allen enorm bedanken voor de inspiratie, de fijne samenwerking, jullie belangstelling en bijdrage aan dit proefschrift. Bindia, Dirk en Margien, veel succes met het afronden van jullie manuscripten, 2015 is ook jullie jaar! Beste $\mathrm{Ad}(\mathrm{je})$, jij bent altijd een van de grote drijfveren geweest achter het thiopurineonderzoek in Amsterdam. Na de pensionering van Leopold Engels, ben jij Orbis komen versterken. Ik wil je in het bijzonder bedanken voor de wijze waarop je mij hebt gesteund, mijn laatste twee manuscripten hebt voorzien van verbeteringen zonder er zelf baat bij te (willen) hebben en me 'klaar te stomen' voor de verdediging. Met jouw komst naar Sittard, hoop ik dat we de onderzoekslijnen met Amsterdam en Maastricht kunnen versterken en dat er in de toekomst nog vele mooie onderzoeken zullen volgen.

De belangrijkste bevindingen van mijn onderzoek zijn voor mij toch wel de studies naar de T1-metingen van 6-TGN en 6-MMPR en de voorspellende waarde ervan voor het optreden van leukopenie en hepatotoxiciteit. De studies werden uitgevoerd in een subgroep van de patiënten van de TOPIC-trial, een groot landelijk multicenter 
farmacogenetisch onderzoek, geinitieerd door het Radboud universitair medisch centrum in Nijmegen.

Beste Corine van Marrewijk, je hebt een hele belangrijke rol gespeeld in de praktische implementatie van het onderzoek bij alle betrokken centra. Bedankt voor je waardevolle ondersteuning tijdens de opstartfase en hulp bij alle onduidelijkheden met betrekking tot priktijden en bewaaromstandigheden van de bloedmonsters. Jouw rol bij het vergaren van de data was onmisbaar. Jij was de spin in het TOPIC-web.

Toen we de onderzoeksvragen bedachten, had ik nooit verwacht dat het ontwikkelen van een voorspellend algoritme (voor mij in ieder geval) zo ingewikkeld zou zijn. Gelukkig kreeg ik hierbij hulp van dr. Marieke Coenen, dr. Sita Vermeulen en Prof. dr. André Verbeek.

Beste Marieke, allereerst wil ik jou bedanken voor het samenstellen van de subTOPIC database. Daarnaast wil ik Sita en jou beiden bedanken voor jullie waardevolle adviezen, de hulp bij de statistische analyses en het schrijven van de manuscripten. Ik heb erg veel van jullie geleerd, dank jullie wel.

Beste André, veel dank voor je hulp bij de ontwikkeling van de predictiemodellen, en de laagdrempelige en humoristische wijze waarop je dat deed. Graag wil ik ook Henk-Jan Guchelaar, Barbara Franke en Dirk de Jong bedanken voor hun waardevolle feedback en aanvullingen op de manuscripten. Ik kijk uit naar de toekomstige onderzoeken die we als TOPIC-study group zullen gaan voortzetten.

Graag wil ik ook Meyno den Dulk, Edy Gemmeke, Aimée Paulissen, Arnold Stronkhorst, Reinhold Stockbrügger, Olaf Klungel, Hans Scheffers, René te Morsche, Wilbert Peters, William Newman, Agnes Veldkamp, Frank Rijmen, Susanne Karner en Matthias Schwab bedanken voor hun bijdragen aan het totstandkomen van dit proefschrift.

Beste Tiny Wouters, in de laatste fase van het gehele traject kwam jij in beeld om alles in een mooie layout te 'gieten'. De snelheid waarmee je dat deed was haast niet bij te houden. Ik vergeet nooit het moment toen ik mijn proefschrift in pdf's ochtends vroeg in mijn mailbox vond, klaar om te versturen naar de beoordelingscommissie. Wow! Tiny, veel dank voor je waardevolle bijdrage, je luisterend oor en adviezen als het even tegenzat, en alle praktische tips die je me gaf die nodig waren om te komen tot dit eindresultaat, bedankt!

Beste Luc en Jeroen Derijks, brothers from another mother, collegae, vrienden, nu zijn jullie aan de beurt.

Mister Deluxe, met mijn komst in Sittard was jouw vertrek helaas een feit. Jouw opleidingsplaats kwam vrij voor mij. In 2005 promoveerde jij al op het onderzoek naar de farmacogenetica en farmacologie van de thiopurines bij de behandeling van IBD. Toen wisten we nog niet dat ik jouw onderzoekslijn zou overnemen. Wie had kunnen denken dat we de jaren erna zo nauw zouden samenwerken, brainstormen, samen congressen zouden bezoeken, zoveel lol zouden hebben, en dat je zelfs mijn paranimf zou zijn? Veel dank voor de mooie tijd en je waardevolle bijdragen aan mijn 
proefschrift. Gelukkig hebben we nog veel stof voor toekomstig onderzoek, ik kijk ernaar uit!

Jeronymus, ook voor mij was het heel bijzonder om samen met jou in opleiding te zijn. Ik kreeg er niet alleen een fijne collega bij, maar ook een goede vriend. We hebben veel aan elkaar gehad, zowel professioneel als privé. Toen je bij ons in opleiding kwam, was je al bezig met jouw promotieonderzoek, wat mij destijds inspireerde om extra onderzoek te doen. Ik zal onze grappen nooit vergeten, wat hebben wij gelachen! Je bent nu gevestigd ziekenhuisapotheker binnen de Coöperatie ZANOB in Brabant. Een functie die toch ook op jouw lijf is geschreven, ook al mis je nu de nauwe contacten met de kliniek. Veel succes met je verdere carrière!

Luc en Jeroen, ik ben trots en dankbaar dat jullie als mijn paranimfen aan mijn flanken zulllen staan om mij mentaal te ondersteunen. Ik hoop dat onze vriendschap lang zal voortduren.

Mijn bijzondere dank gaat uit naar mijn collegae van de ziekenhuisapotheek van het Orbis Medisch Centrum.

Eind 2003 kwam ik op solliciatiegesprek bij drie gepromoveerde ziekenhuisapothekers Piet Hooymans, Rob Janknegt en Sjef Lohman in het Maaslandziekenhuis te Sittard. Nooit verwachtende dat ik überhaupt een kans maakte op de opleidingsplaats, gezien mijn beperkte ervaring met onderzoek. Wat een leerzame en leuke opleiding heb ik onder jullie hoede mogen genieten. Jullie interesse voor onderzoek is mij met de 'paplepel' ingegoten. Ik ben dan ook trots dat ik straks in het rijtje sta van de door jullie opgeleide Zitterdse gepromoveerde ziekenhuisapothekers.

Beste Sjef, rustig en weloverwogen als je altijd was, ik waardeerde het altijd enorm hoe je de tijd nam om samen met mij een farmaceutisch probleem uit te zoeken. Daar heb ik veel van geleerd. Bedankt voor de mooie opleidingstijd en je bijdragen aan mijn registratieonderzoeken.

Lieve dames van de apotheek, toen ik met de opleiding startte in Zitterd, werd ik warm onthaald. Wat waren jullie lief voor mij! Enorm bedankt voor de gezellige opleidingstijd, en de tijd erna natuurlijk, voor de heerlijke snoeperijen (ok, ok, ik zal ook weer eens wat lekkers meebrengen!), voor jullie oprechte interesse, steun en gezellige afleiding op de werkvloer.

Allerbeste analisten van ons laboratorium, Jean Cilissen, Mariëlle Maas, Milevis Reitsma, Jean-Pierre Bollen, Miet Fiddelaers en Leonie Peters, de leerzame en gezellige tijd op het lab tijdens mijn opleiding (en erna) zal ik niet vergeten. Jullie leerden mij de fijne kneepjes van het farmaceutisch analytische vak. Ontzettend bedankt voor de ondersteuning en de thiopurine bepalingen die jullie hebben gedaan - niet alleen in het kader van mijn onderzoek, maar ook van de talloze patiëntenmonsters komende uit heel Nederland. Mede dankzij jullie is het Klinisch farmacologisch \& Toxicologisch laboratorium van Sittard één van de belangrijkste specialistische laboratoria van Nederland geworden op het gebied van de thiopurine-bepaling bij de behandeling van 
inflammatoire darmziekten. Beste Bas en Bete, fijn dat jullie ons analistenteam hebben versterkt. Jullie ook bedankt voor de goede ondersteuning en fijne samenwerking.

Beste Jean, jou wil ik graag in het bijzonder bedanken voor je steun en de vakkundige wijze waarop je de Lennard-, en later de Dervieux-methode hebt opgezet, maar ook voor de vele monsters die je voor ons hebt bepaald. Je bent echt een vakspecialist! Ik hoop dat je snel weer aansterkt en straks weer in volle gezondheid in het leven kunt staan.

Beste Rob Janknegt, Brigit van Oijen, Niels Boone, Kobra Waizy, Hugo de Wit, Zita Breukers, Hugo van der Kuy en collega ziekenhuisapothekers in opleiding, Carlota Mestres Gonzalvo en Fieke van Moorsel, ik realiseer me dat ik het laatste jaar door de drukte in mijn hoofd (en misschien een beetje slaapgebrek) mogelijk niet altijd even toegankelijk was. Graag wil ik jullie bedanken voor het begrip, de getoonde interesse en de ruimte die jullie mij hebben gegeven om mijn proefschrift af te ronden. Ik bof met zulke lieve collegae, bedankt voor de fijne samenwerking.

Brigit, ik hoop dat je snel naast me kunt komen staan in het rijtje Zitterdse gepromoveerde ziekenhuisapothekers. Promoveren en trouwen, of andersom? Het maakt niet uit: 2015 is jouw jaar!

Hugo (de Wit), Niels en Carlota, jullie zijn ook al met het promotie-virus besmet geraakt. Veel succes gewenst met de afronding ervan! Fieke, pas jij maar op, bij jou is de incubatietijd al ingegaan!

Beste Rob, ook jou wil ik graag bedanken voor de leerzame opleidingstijd, de fijne samenwerking, je support, het lezen/corrigeren van mijn manuscripten en daarmee bijdrage aan mijn proefschrift.

Beste Hugo (van der Kuy), een grote bewondering heb ik voor jouw innovatieve inzichten in de ziekenhuisfarmacie, jouw helicopter-view en de kansen die je ziet om verbetering van de patiëntenzorg te koppelen aan nieuw onderzoek. De afgelopen jaren heb je mij geïnspireerd om mezelf verder te ontwikkelen. Je hebt mij tegen mezelf beschermd, daar waar ik het zelf niet zag. Daarvoor ben ik je erg dankbaar. Ik ben er trots op dat wij, als discipelen van dr. Hooymans en dr. Lohman, het opleiderschap hebben mogen overnemen. Bedankt voor alles wat je voor mij hebt gedaan.

Mijn lieve familie, en al mijn lieve vrienden, bedankt voor jullie support, de getoonde interesse en voor de gezellige afleiding die jullie ons de afgelopen jaren hebben bezorgd. Na het afronden van dit promotietraject is er eindelijk meer tijd voor qualitytime.

Lieve familie Van Wylick en familie Zonderland, jullie ook veel dank voor de keer op keer oprechte interesse, gezelligheid en warmte die je ons de afgelopen jaren hebben gegeven.

'My homies' uit 'New Joke City' (Nieuwegein, voor de minder ingewijden) Niels, Iris, Jefron, Marcel, Lex en Dennis, wat een mooie jeugd hebben wij samen gehad! Jullie vriendschap heeft in belangrijke mate bijgedragen aan degene die ik nu ben. De afstand heeft absoluut geen invloed gehad op onze vriendschap, behalve dat we elkaar vééééél 
te weinig zien natuurlijk. Ik ben enorm dankbaar voor jullie vriendschap door de jaren heen - dat deze nog lang moge voortduren.

Marcel(o), superbedankt voor je creativiteit en professionaliteit waarmee je de cover van mijn proefschrift hebt vormgegeven. Je bent een man van veel talenten en kwaliteiten, een autodidacticus in hart en nieren en een hele trouwe vriend. Hopelijk vind je spoedig 'het gouden ei', waarmee je creativiteit wordt beloond.

Kevin(ho), jij ook enorm bedankt voor je vriendschap en het maken van de foto. Je weet sinds een paar jaar eindelijk welke richting je op moet gaan. Heel veel succes met het afronden van de fotoacademie, je bent/wordt een topfotograaf!

Mijn Brazilian Jiu Jitsu maatjes, in het bijzonder Tom, Dennis, Niek, Bart, Fredrik, Christophe, Rudy, Wouter en Tim wil ik graag bedanken voor de fysieke en mentale afleiding op de mat, al had ik daar de laatste jaren helaas veel te weinig tijd voor. De BJJ-trainingen waren de enige momenten dat ik de drukte echt even los kon laten, en mentaal kon ontspannen. Mijn oneindige, onverklaarbare passie en fascinatie voor de vechtkunst kan ik met jullie delen, dank jullie wel! Op naar de Brazilian Black Belt, Osu!

Lieve Alphons en Liesbeth, Jochen en Shelly, Hendrik-Jan en Myrte, wat heb ik geboft met jullie als schoonfamilie. De laatste jaren zijn jullie voor ons een enorme steun geweest. Als ook Emmely haar studie straks heeft afgerond, is er weer meer tijd om leuke dingen met elkaar te ondernemen. Heel veel dank voor jullie oprechte interesse, steun en warmte de afgelopen jaren.

Lieve Oma Els, elke keer als we bij u op bezoek kwamen, bleek dat ook u vanuit Utrecht het gehele traject nauw volgde. Uw vitaliteit op 94-jarige leeftijd is bewonderenswaardig. Gezien de afstand en de bijbehorende hectiek kunt u helaas niet bij de promotie aanwezig zijn, maar Emmely en ik komen snel weer bij $u$ op bezoek om dit heugelijke feit met $u$ te vieren. Ik ben heel dankbaar voor uw betrokkenheid en geniet elke keer weer van onze gesprekken.

Lieve Robin en Véronique, lieve Janneke en Laurens, wat heb ik het toch getroffen met zo'n broer en zus, schoonzus en zwager. Jullie hebben het gehele promotietraject van een wat grotere afstand meegekregen. Desondanks ben ik dankbaar voor jullie getoonde belangstelling en hebben jullie regelmatig voor gezellige afleiding gezorgd. Gelukkig komen er nu weer wat rustigere tijden aan, en zullen we weer vaker bij elkaar zijn. Ik kijk uit naar alle gezellige en mooie momenten die nog komen gaan.

Lieve Papa, mede dankzij jou ben ik farmacie gaan studeren. Na mijn studie heb ik eerst bij jou in de apotheek mogen werken, waarna ik besloot om mijn 'vleugels uit te slaan'. Uiteindelijk heb ik gekozen voor de ziekenhuisfarmacie, een keuze die je nooit hebt geprobeerd te beïnvloeden, ook al had je het misschien diep in je hart wel anders willen zien. Ik heb jou altijd beschouwd als een apotheker in hart en nieren, met een enorme hoeveelheid farmaceutische kennis en een ijzersterk geheugen. Je stond altijd voor 
iedereen klaar en nam altijd de tijd om de patiënten te helpen waar je kon, zowel ín de apotheek als erbuiten. Jouw algemene ontwikkeling en technische kennis hebben mij altijd enorm geïnspireerd. Medio 2013 ben je met pensioen gegaan, dubbel en dwars verdiend!

Lieve Mutti, jíj bent altijd de sterke vrouw geweest die naast haar hardwerkende man stond. Onze opvoeding heb je voor een groot deel voor jouw rekening genomen wat geen gemakkelijke taak voor je was, gezien onze verschillende karakters. Ik ben je enorm dankbaar voor de wijze waarop je ons hebt opgevoed. De stelling van Maya Angelou deed me erg aan jou denken, vandaar dat ik deze heb gekozen voor mijn proefschrift.

Lieve Papa en Mama, bedankt voor jullie onvoorwaardelijke steun, aanwezigheid, geloof en liefde, en de mogelijkheden die jullie mij hebben geboden om te worden tot wie ik nu ben. Geniet samen van deze mooie periode van het leven. Hopelijk mogen we nog lang in goede gezondheid van elkaar en ons groeiende gezin genieten.

Allerliefste Emmely, mijn zonnetje in huis, mijn rots in de branding, wat ben ík gelukkig dat jíj in mijn leven bent gekomen! Woorden kunnen niet beschrijven hoe dankbaar ik ben voor jouw onvoorwaardelijke steun, liefde, begrip, interesse, geduld en onmisbare hulp in álle opzichten. Jouw frisse heldere blik en goeie humor gaven mij telkens weer moed om door te gaan. Ook jij bent je al 3,5 jaar aan het bijten in je tweede studie. Ik ben ongelofelijk trots op je, meiske, je bent er bijna! Nog even en dan gaan we samen onze nieuwe levensfase in, youandi!

Emmelytje, ik hou ontzettend veel van jou!

Dennis

Geleen, november 2014 
List of publications 



\section{List of publications}

DR Wong, MJH Coenen, LJ Derijks, SH Vermeulen, CJ van Marrewijk, OH Klungel, H Scheffer, B Franke, HJ Guchelaar, DJ de Jong, LGJB Engels, ALM Verbeek, PM Hooymans. Early elevated 6-methylmercaptopurine levels predict thiopurine-induced hepatotoxicity in inflammatory bowel disease. Submitted for publication

DR Wong, MJH Coenen, SH Vermeulen, LJ Derijks, CJ van Marrewijk, OH Klungel, H Scheffer, B Franke, HJ Guchelaar, DJ de Jong, LGJB Engels, ALM Verbeek, PM Hooymans, on behalf of the TOPIC consortium. Early assessment of thiopurine metabolites identifies patients at risk of leukopenia in inflammatory bowel disease. Submitted for publication.

MJ Coenen, DJ de Jong, CJ van Marrewijk, LJ Derijks, SH Vermeulen, DR Wong, OH Klungel, ALM Verbeek, PM Hooymans, WHM Peters, RHM te Morsche, WG Newman, H Scheffer, HJ Guchelaar, B Franke. Screening for TPMT genotype followed by personalized dosing of azathioprine and mercaptopurine in inflammatory bowel disease patients: a randomized trial. Submitted for publication.

DR Wong, M Pierik, ML Seinen, AA van Bodegraven, LP Gilissen, P Bus, JA Bakker, AAM Masclee, C Neef, LGJB Engels, PM Hooymans. The pharmacokinetic effect of adalimumab on thiopurine metabolism in Crohn's disease patients. J Crohns Colitis. 2014;8:120-8.

DR Wong ${ }^{*}$, LPL Gilissen*, LGJB Engels, J Bierau, JA Bakker, AD Paulussen, MJ RombergCamps, A Stronkhorst, P Bus, LP Bos, PM Hooymans, RW Stockbrügger, C Neef, AAM Masclee. Therapeutic drug monitoring of thiopurine metabolites in adult thiopurine tolerant IBD patients on maintenance therapy. J Crohns Colitis 2012;6:698-707. " equally contributed

LJ Derijks, DR Wong. Pharmacogenetics of thiopurines in inflammatory bowel disease. Curr Pharm Des. 2010;16:145-54. Review.

P de Graaf, NK de Boer, DR Wong, S Karner, B Jharap, PM Hooymans, Al Veldkamp, CJ Mulder, AA van Bodegraven, $M$ Schwab. Influence of 5-aminosalicylic acid on 6-thioguanosine phosphate metabolite levels: a prospective study in patients under steady thiopurine therapy. Br J Pharmacol. 2010;160:1083-91.

WGN Mares, DR Wong L LPL Gilissen, AAM Masclee, PM Hooymans, LGJB Engels. Safe 6-thioguanine therapy of a TPMT deficient Crohn's disease patient by using therapeutic drug monitoring (case report). J Crohns Colitis 2009;3:128-30. 
DR Wong, NKH de Boer, B Jharap, P de Graaf, AA van Bodegraven, CJJ Mulder, LGJB Engels, F Rijmen, PM Hooymans. De invloed van mesalazine op het thiopurine metabolisme bij IBD-patienten: een prospectief multicenter farmacokinetisch onderzoek. Pharm Weekblad 2008; 2:62-65.

DR Wong, LJ Derijks, MO den Dulk, EHKM Gemmeke, PM Hooymans. The role of xanthine oxidase in thiopurine metabolism: a case report. Ther Drug Monit. 2007; 29:845-848.

NKH de Boer, DR Wong, B Jharap, P de Graaf, PM Hooymans, CJJ Mulder, F Rijmen, LGJB Engels, AA van Bodegraven. Dose-dependent influence of 5-aminosalicylates on thiopurine metabolism. Am J Gastroenterol. 2007;102:2747-53.

B Panis, DR Wong, PM Hooymans, PAGM De Smet, PP Rosias. Recurrent toxic hepatitis in a Caucasian girl related to the use of Shou-Wu-Pian, a Chinese herbal preparation. J Pediatr Gastroenterol Nutr. 2005; 41:256-8. 




\section{Curriculum vitae (EN)}

Dennis Wong was born on November 29, 1975 in Amersfoort, the Netherlands. In 1994, he started the study of Pharmacy at Utrecht University. In 1999, he obtained his Master degree of Pharmacy, and became a pharmacist in 2001. After his graduation, he worked for 6 months at his father's pharmacy 'De Liefde's Centraal apotheek' in Utrecht. Subsequently, he worked at the Ikazia Hospital and later at the Rijnmond-Zuid Medical Centre, both of which are situated in Rotterdam. In February 2004, he started his Hospital pharmacy residency training under the supervision of Dr. P.M. Hooymans and Dr. J.J.H.M. Lohman, at the Department of Clinical Pharmacy \& Toxicology at Maasland Hospital in Sittard, the Netherlands.

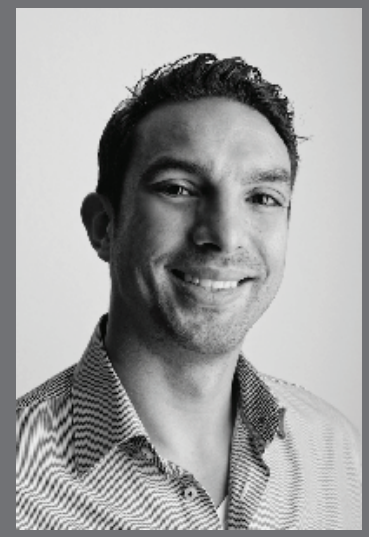

After having become a registered hospital pharmacist in 2008 , he then embarked on the Clinical Pharmacology training at the same hospital until the end of 2009 . The clinical studies performed in the course of these training programs form the foundation of this thesis. Since October 2009, he remains a staff member of the Department of Clinical Pharmacy \& Toxicology at the Orbis Medical Centre (formerly Maasland hospital), Sittard-Geleen.

\section{Curriculum vitae (NL)}

Dennis Wong werd geboren op 29 november 1975 te Amersfoort. In 1994 begon hij met de studie Farmacie aan de Universiteit Utrecht. In 1999 werd het doctoraal Farmacie behaald, en in 2001 het Apothekersexamen.

Na een half jaar bij zijn vader in De Liefde's Centraal apotheek te Utrecht te hebben gewerkt, deed Dennis werkervaring op als projectapotheker in het Ikazia Ziekenhuis, en later in het Medisch Centrum Rijnmond-Zuid, beiden te Rotterdam.

In 2004 startte hij onder begeleiding van dr. P.M. Hooymans en dr. J.J.H.M. Lohman met de opleiding tot ziekenhuisapotheker in het Maaslandziekenhuis te Sittard. Na de registratie als ziekenhuisapotheker in 2008 werd gestart met de opleiding tot klinisch farmacoloog, die eind 2009 werd afgerond. De onderzoeken die in het kader van deze specialisaties werden uitgevoerd, vormen de basis van dit proefschrift.

Sinds oktober 2009 is hij als staflid werkzaam in het Orbis Medisch Centrum (voorheen Maaslandziekenhuis), afdeling Klinische Farmacie \& Toxicologie te Sittard-Geleen. 